\title{
FLIGHT AND STABILITY OF A LASER INERTIAL FUSION ENERGY TARGET IN THE DRIFT REGION BETWEEN INJECTION AND THE REACTION CHAMBER WITH COMPUTATIONAL FLUID DYNAMICS
}

\author{
A Thesis \\ presented to \\ the Faculty of California Polytechnic State University, \\ San Luis Obispo
}

\author{
In Partial Fulfillment \\ of the Requirements for the Degree \\ Master of Science in Mechanical Engineering
}

By

Tiffany Leilani Mitori

March 2014 


\section{LLNL-TH-647698}

Disclaimer

This document was prepared as an account of work sponsored by an agency of the United States government. Neither the United States government nor Lawrence Livermore National Security, LLC, nor any of their employees makes any warranty, expressed or implied, or assumes any legal liability or responsibility for the accuracy, completeness, or usefulness of any information, apparatus, product, or process disclosed, or represents that its use would not infringe privately owned rights. Reference herein to any specific commercial product, process, or service by trade name, trademark, manufacturer, or otherwise does not necessarily constitute or imply its endorsement, recommendation, or favoring by the United States government or Lawrence Livermore National Security, LLC. The views and opinions of authors expressed herein do not necessarily state or reflect those of the United States government or Lawrence Livermore National Security, LLC, and shall not be used for advertising or product endorsement purposes.

This work performed under the auspices of the U.S. Department of Energy by Lawrence Livermore National Laboratory under Contract DE-AC52-07NA27344.

(C) 2014

Tiffany Leilani Mitori

ALL RIGHTS RESERVED 


\section{COMMITTEE MEMBERSHIP}

TITLE:

Flight and Stability of a Laser Inertial Fusion Energy

Target in the Drift Region between Injection and the

Reaction Chamber with Computational Fluid Dynamics

AUTHOR: $\quad$ Tiffany Leilani Mitori

DATE SUBMITTED: $\quad$ March 2014

COMMITTEE CHAIR: $\quad$ Dr. Kim Shollenberger, Mechanical Engineering Professor at California Polytechnic State University

COMMITTEE MEMBER: Dr. Patrick Lemieux, Mechanical Engineering Associate Professor at California Polytechnic State University

COMMITTEE MEMBER: Dr. James Scott Patton, Mechanical Engineering Associate Professor at California Polytechnic State University

COMMITTEE MEMBER: Dr. Mark Havstad, Engineering at Lawrence Livermore National Laboratory 


\begin{abstract}
Flight and Stability of a Laser Inertial Fusion Energy Target in the Drift Region between Injection and the Reaction Chamber with Computational Fluid Dynamics Software Tiffany Leilani Mitori
\end{abstract}

A Laser Inertial Fusion Energy (LIFE) target's flight through a low Reynolds number and high Mach number regime was analyzed with computational fluid dynamics software. This regime consisted of xenon gas at 1,050 K and approximately 6,670 Pa. Simulations with similar flow conditions were performed over a sphere and compared with experimental data and published correlations for validation purposes. Transient considerations of the developing flow around the target were explored. Simulations of the target at different velocities were used to determine correlations for the drag coefficient and Nusselt number as functions of the Reynolds number. Simulations with different target angles of attack were used to determine the aerodynamic coefficients of drag, lift, Magnus moment, and overturning moment as well as target stability. The drag force, lift force, and overturning moment changed minimally with spin. Above an angle of attack of $15^{\circ}$, the overturning moment would be destabilizing. At angles of attack less than $15^{\circ}$, the overturning moment would tend to decrease the target's angle of attack, indicating the lack of a need for spin for stability at these small angles. This stabilizing moment would cause the target to move in a mildly damped oscillation about the axis parallel to the free-stream velocity vector through the target's center of gravity.

Keywords: CFD, low Reynolds number, high Mach number, drag coefficient correlation, Nusselt correlation, angle of attack, aerodynamic coefficients, flight stability. 


\section{ACKNOWLEDGMENTS}

The author would like to thank Lawrence Livermore National Laboratory (LLNL) for sponsoring the thesis. The author would also like to thank ANSYS customer support for providing temporary high-performance-computing licenses and Don at Livermore Computing for installing ANSYS on the LLNL clusters. 3D simulations would have been impossible without their help. The author would also like to thank Paul and the rest

of the Laser Inertial Fusion Energy (LIFE) team for their support and guidance. Finally, the author wishes to thank Dr. Kim Shollenberger and Dr. Mark Havstad for their mentorship, advice, and support over the duration of the project. Their help has been invaluable in the completion of this thesis. 


\section{TABLE OF CONTENTS}

Page

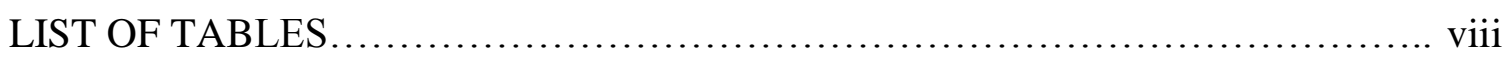

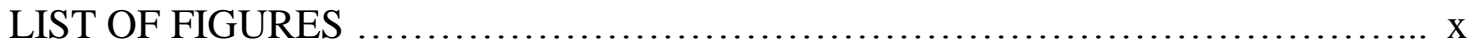

\section{CHAPTER}

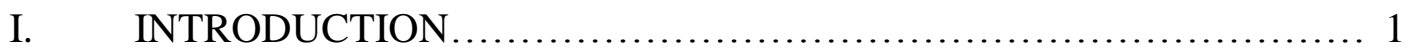

The National Ignition Facility (NIF) and Laser Inertial Fusion Energy

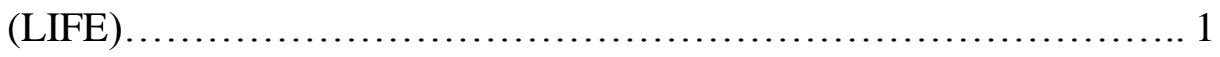

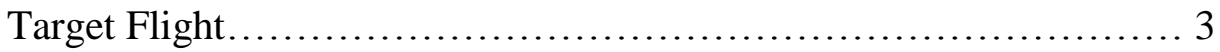

List of Terms..................................................

II. BACKGROUND ................................................. 10

Xenon Properties.......................................... 10

External Flow over a Sphere................................. 14

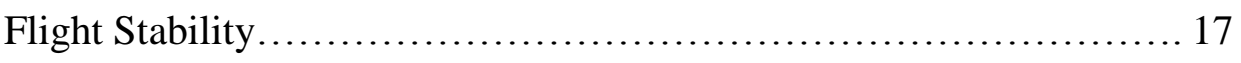

III. COMPUTATIONAL FLUID DYNAMICS (CFD) ..................... 20

Sphere Validation: Fluent Setup $\ldots \ldots \ldots \ldots \ldots \ldots \ldots \ldots \ldots \ldots \ldots \ldots \ldots \ldots \ldots \ldots$

Sphere Validation: Domain Size and Mesh Refinement Studies........ 24

2D, Axisymmetric Drift Regime: Fluent Setup..................... 30

2D, Axisymmetric Drift Regime: Domain Size, Mesh Refinement, and

Transient Considerations..................................... 33

3D Drift Regime: Fluent Setup............................... 39

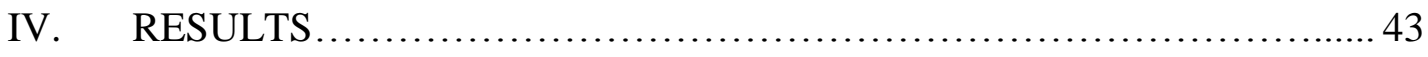

Sphere Validation: Results..................................43 
2D, Axisymmetric Drift Regime: Results....

3D Drift Regime: Results..................................68

V. CONCLUSIONS ................................................ 88

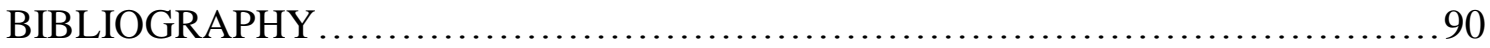

APPENDICES

A. Fluent Setup............................................ 94

B. Additional Plots of 2D, Axisymmetric Swirl, Transient Simulation of Target in Drift Region..................................... 100

C. Additional Plots of 3D, Steady-State Simulations of Target in Drift Region............................................... 104

D. Angle of Attack MATLAB Code and Plots........................130 


\section{LIST OF TABLES}

Table

Page

1. Summary of LIFE Target Injection Regimes.............................. 5

2. Gaseous Xenon Viscosity and Thermal Conductivity at Selected Temperatures

Determined by Kinetic Theory..................................... 13

3. Estimates for Target Mass and Moments of Inertia......................... 19

4. Input Parameters for Sphere Validation Cases with Different Reynolds

Numbers

5. Xenon Properties for Sphere Validation Simulations in Drift Region at 1,050

$\mathrm{K}$ and 6,666 $\mathrm{Pa}$

6. Sphere Domain Size Study.

7. Sphere Mesh Refinement Study .................................... 27

8. Xenon Properties in Drift Region for Target Simulations.................... 31

9. Domain Study on 2D, Axisymmetric Swirl, Transient Drift Flow over

Target.

10. Mesh Refinement Study on 2D, Axisymmetric Swirl, Transient Drift Flow

over Target...................................................... 36

11. Time Step Size Study on 2D, Axisymmetric Swirl, Transient Drift Flow over

Target.

12. Velocities and Mach Numbers for 3D, Steady-State Target Simulations with

Different Reynolds Numbers.

13. 3D, Steady-State Target Spin Axis for Varying Angle of Attack Cases.

14. Sphere Drag Coefficient Comparison. 
15. Sphere Separation Angles (from Front Stagnation Point to Separation Point) from Fluent Simulations and Clift Correlation at Different Reynolds

Numbers

16. Drag Force and Heat Transfer on Target after $1 \mathrm{~ms}$ from 2D, Axisymmetric Swirl Model

17. Drag Force and Heat Transfer on Target from 3D, Steady-State Target Model

18. Drag and Heat Transfer Coefficient Percent Difference between Transient, 2D, Axisymmetric Swirl Model and 3D, Steady-State Model....

19. Drag and Heat Transfer from 3D, Steady-State Target Simulations at Various Reynolds Numbers .78

20. Aerodynamic Coefficients and Static Stability of Target in Drift Region at Different Angles of Attack. .86

21. Angle of Attack Oscillation and Exit Value Determined by MATLAB Code... 87

22. Detailed Fluent Case Setup for 2D, Axisymmetric Sphere in Drift Region.....94

23. Detailed Fluent Case Setup for 2D, Axisymmetric Swirl Target in Drift Region

24. Detailed Fluent Case Setup for 3D, Steady-State Target in Drift Region 97 


\section{LIST OF FIGURES}

Figure

Page

1. Picture of NIF Target; Credit: Lawrence Livermore National Laboratory $[15]$

2. Simulated Image of Lasers Heating NIF Target Hohlraum; Credit: Lawrence

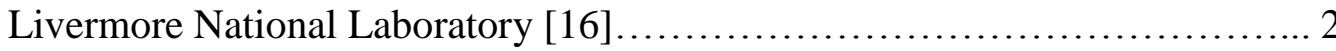

3. LIFE Reaction Chamber Depicting Lasers and Target Injection Path; Credit:

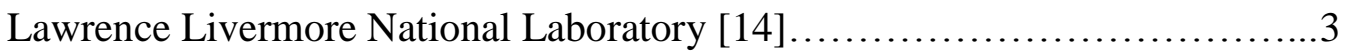

4. LIFE Target Geometry with Dimensions (Not Drawn to Scale)............... 4

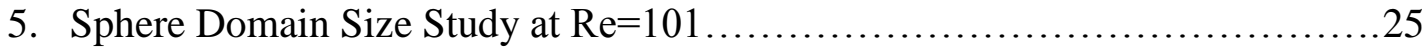

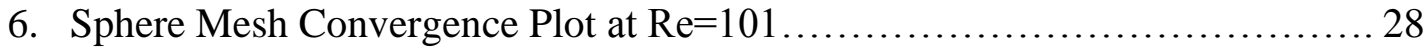

7. Selected Mesh (Case 6 from Refinement Table) for Sphere Simulations......... 28

8. Inflation Layers of Selected Mesh (Case 6 from Refinement Table) for Sphere Simulations.

9. 2D, Axisymmetric Swirl Target Mesh Convergence Plot........................ 36

10. Entire View of 2D, Axisymmetric Target Mesh............................ 37

11. Inflation Layer View of 2D, Axisymmetric Target Mesh..................... 37

12. Zoomed-in View of Target in 3D Target Mesh Sliced at X-Y Plane............ 40

13. Inflation Layer View of 3D Target Mesh Sliced at X-Y Plane................ 41

14. Sphere Drag Coefficient versus Reynolds Number for Experimental Data, a Published Correlation, and Fluent Simulation Results...................... 44

15. Graph of Percent Difference between Fluent Results and Experimental Data and a Published Correlation 
16. Wall Shear Stress Plot on Sphere at Different Reynolds Numbers.............. 46

17. Sphere Velocity Contour with Streamlines Overlaid at $\mathrm{Re}=21.1 \ldots \ldots \ldots \ldots \ldots . \ldots 48$

18. Sphere Velocity Contour with Streamlines Overlaid at $\mathrm{Re}=23.4 \ldots \ldots \ldots \ldots \ldots . .49$

19. Sphere Velocity Contour with Streamlines Overlaid at $\mathrm{Re}=29.1 \ldots \ldots \ldots \ldots \ldots . .49$

20. Sphere Velocity Contour with Streamlines Overlaid at $\mathrm{Re}=45 \ldots \ldots \ldots \ldots \ldots \ldots . . \ldots 49$

21. Sphere Velocity Contour with Streamlines Overlaid at $\mathrm{Re}=50.6 \ldots \ldots \ldots \ldots \ldots \ldots . \ldots 50$

22. Sphere Velocity Contour with Streamlines Overlaid at $\mathrm{Re}=54.4 \ldots \ldots \ldots \ldots \ldots . \ldots 50$

23. Sphere Velocity Contour with Streamlines Overlaid at $\mathrm{Re}=68.9 \ldots \ldots \ldots \ldots \ldots . \ldots 50$

24. Sphere Velocity Contour with Streamlines Overlaid at $\mathrm{Re}=78.2 \ldots \ldots \ldots \ldots \ldots . \ldots 51$

25. Sphere Velocity Contour with Streamlines Overlaid at $\mathrm{Re}=88.1 \ldots \ldots \ldots \ldots \ldots . \ldots 51$

26. Sphere Velocity Contour with Streamlines Overlaid at $\mathrm{Re}=93.8 \ldots \ldots \ldots \ldots \ldots . \ldots 51$

27. Sphere Velocity Contour with Streamlines Overlaid at $\mathrm{Re}=101 \ldots \ldots \ldots \ldots \ldots \ldots . \ldots 52$

28. Sphere Velocity Contour with Streamlines Overlaid at $\mathrm{Re}=104 \ldots \ldots \ldots \ldots \ldots \ldots . \ldots 52$

29. Sphere Velocity Contour with Streamlines Overlaid at $\mathrm{Re}=108 \ldots \ldots \ldots \ldots \ldots \ldots . \ldots 2$

30. Sphere Velocity Contour with Streamlines Overlaid at $\operatorname{Re}=109 \ldots \ldots \ldots \ldots \ldots \ldots . \ldots 3$

31. Sphere Velocity Contour with Streamlines Overlaid at $\mathrm{Re}=124 \ldots \ldots \ldots \ldots \ldots \ldots . \ldots 3$

32. Sphere Velocity Contour with Streamlines Overlaid at $\operatorname{Re}=130 \ldots \ldots \ldots \ldots \ldots \ldots . \ldots 3$

33. Zoomed-in View of Sphere Velocity Contour with Streamlines Overlaid at

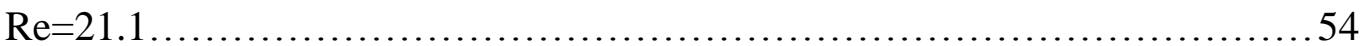

34. Zoomed-in View of Sphere Velocity Contour with Streamlines Overlaid at

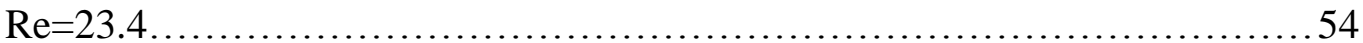

35. Velocity Contour and Streamlines of 2D, Axisymmetric Swirl Flow over

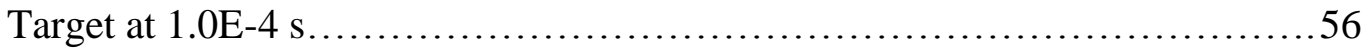


36. Velocity Contour and Streamlines of 2D, Axisymmetric Swirl Flow over Target at $2.0 \mathrm{E}-4 \mathrm{~s}$

37. Velocity Contour and Streamlines of 2D, Axisymmetric Swirl Flow over

Target at $3.0 \mathrm{E}-4 \mathrm{~s}$

38. Velocity Contour and Streamlines of 2D, Axisymmetric Swirl Flow over

Target at $4.0 \mathrm{E}-4 \mathrm{~s}$

39. Velocity Contour and Streamlines of 2D, Axisymmetric Swirl Flow over

Target at 5.0E-4 s.... .58

40. Velocity Contour and Streamlines of 2D, Axisymmetric Swirl Flow over

Target at 6.0E-4 s

41. Velocity Contour and Streamlines of 2D, Axisymmetric Swirl Flow over

Target at $7.0 \mathrm{E}-4 \mathrm{~s}$

42. Velocity Contour and Streamlines of 2D, Axisymmetric Swirl Flow over

Target at $8.0 \mathrm{E}-4 \mathrm{~s}$

43. Velocity Contour and Streamlines of 2D, Axisymmetric Swirl Flow over

Target at $9.0 \mathrm{E}-4 \mathrm{~s}$

44. Velocity Contour and Streamlines of 2D, Axisymmetric Swirl Flow over

Target at $1.0 \mathrm{E}-3 \mathrm{~s}$

45. Target Drag Force over Time in Drift Region from 2D, Axisymmetric Swirl

Model

46. Target Heat Transfer over Time in Drift Region from 2D, Axisymmetric Swirl

Model 
47. Mach Number around Target in Drift Region from 2D, Axisymmetric Swirl Model.

48. Absolute Pressure around Target in Drift Region from 2D, Axisymmetric Swirl Model. 65

49. Turbulent Intensity around Target in Drift Region from 2D, Axisymmetric

Swirl Model 66

50. Total Surface Heat Flux around Target in Drift Region from 2D, Axisymmetric

Swirl Model........................................................ 66

51. Velocity Contour and Streamline Plot for Target in 3D, Steady-State, Drift

Simulation

52. Mach Number around Target from 3D, Steady-State Simulation. 70

53. Absolute Pressure around Target from 3D, Steady-State Simulation........... 70

54. Turbulent Intensity around Target from 3D, Steady-State Simulation...........71

55. Total Surface Heat Flux around Target from 3D, Steady-State Simulation...... 71

56. Drag Coefficient Comparison between Transient, 2D, Axisymmetric Swirl Model and 3D, Steady-State Model. ...

57. Heat Transfer Coefficient Comparison between Transient, 2D, Axisymmetric Swirl Model and 3D, Steady-State Model. .73

58. Heat Flux from 3D, Steady-State Case Plotted with Published Heat Transfer Correlations .76

59. Drag Coefficient Plotted for 3D, Steady-State Target Simulations at Various Reynolds Numbers. .78 
60. Nusselt Number Plotted for 3D, Steady-State Target Simulations at Various Reynolds Numbers.

61. Velocity Contour and Streamline Plot for Target in 3D, Steady-State, Drift Simulation with $1^{\circ}$ Angle of Attack. 80

62. Velocity Contour and Streamline Plot for Target in 3D, Steady-State, Drift Simulation with $2^{\circ}$ Angle of Attack. 81

63. Velocity Contour and Streamline Plot for Target in 3D, Steady-State, Drift Simulation with $5^{\circ}$ Angle of Attack. 81

64. Velocity Contour and Streamline Plot for Target in 3D, Steady-State, Drift Simulation with $10^{\circ}$ Angle of Attack.

65. Velocity Contour and Streamline Plot for Target in 3D, Steady-State, Drift Simulation with $15^{\circ}$ Angle of Attack.

66. Velocity Contour and Streamline Plot for Target in 3D, Steady-State, Drift Simulation with $20^{\circ}$ Angle of Attack.

67. Forces on Spinning and Non-Spinning Target in Drift Region at Different Angles of Attack from 3D, Steady-State Model .84

68. Moments on Spinning and Non-Spinning Target in Drift Region at Different Angles of Attack from 3D, Steady-State Model. .84

69. Pressure Contour of 2D, Axisymmetric Swirl Flow over Target in Drift Region at $1.0 \mathrm{E}-3 \mathrm{~s}$. 100

70. Temperature Contour of 2D, Axisymmetric Swirl Flow over Target in Drift Region at $1.0 \mathrm{E}-3 \mathrm{~s}$. 
71. Total Temperature around Target in Drift Region from 2D, Axisymmetric Swirl Model. 101

72. Wall Shear Stress around Target in Drift Region from 2D, Axisymmetric Swirl

Model.

73. Turbulent Dissipation Rate around Target in Drift Region from 2D, Axisymmetric Swirl Model

74. Turbulent Kinetic Energy around Target in Drift Region from 2D,

Axisymmetric Swirl Model

75. Y+ Values around Target in Drift Region from 2D, Axisymmetric Swirl

Model

76. Pressure Contour of 3D, Steady-State Spinning Target in Drift Region 104

77. Temperature Contour of 3D, Steady-State Spinning Target in Drift Region.

78. Total Temperature around Target in Drift Region from 3D, Steady-State Model 105

79. Wall Shear Stress around Target in Drift Region from 3D, Steady-State

Model

80. Turbulent Dissipation Rate around Target in Drift Region from 3D, Steady-

State Model. 106

81. Turbulent Kinetic Energy around Target in Drift Region from 3D, Steady-State Model 106

82. Y+ Values around Target in Drift Region from 3D, Steady-State Model. .107

83. Pressure Contour of 3D, Steady-State, Spinning Target in Drift Region at a Reynolds Number of 2,400 107 
84. Temperature Contour of 3D, Steady-State, Spinning Target in Drift Region at

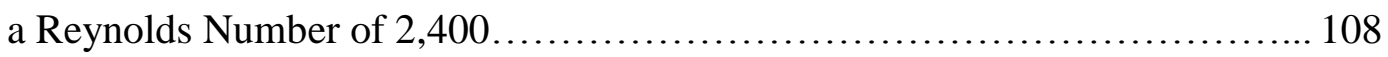

85. Velocity Contour with Streamlines of 3D, Steady-State, Spinning Target in

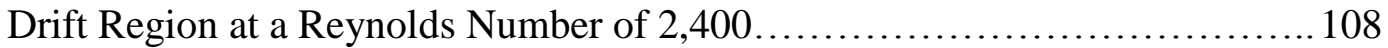

86. Pressure Contour of 3D, Steady-State, Spinning Target in Drift Region at a Reynolds Number of 2,800 109

87. Temperature Contour of 3D, Steady-State, Spinning Target in Drift Region at

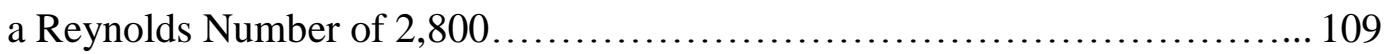

88. Velocity Contour with Streamlines of 3D, Steady-State, Spinning Target in Drift Region at a Reynolds Number of 2,800.

89. Pressure Contour of 3D, Steady-State, Spinning Target in Drift Region at a Reynolds Number of 3,200

90. Temperature Contour of 3D, Steady-State, Spinning Target in Drift Region at a Reynolds Number of 3,200.

91. Velocity Contour with Streamlines of 3D, Steady-State, Spinning Target in Drift Region at a Reynolds Number of 3,200.

92. Pressure Contour of 3D, Steady-State, Spinning Target in Drift Region at a Reynolds Number of 3,600

93. Temperature Contour of 3D, Steady-State, Spinning Target in Drift Region at a Reynolds Number of 3,600.

94. Velocity Contour with Streamlines of 3D, Steady-State, Spinning Target in Drift Region at a Reynolds Number of 3,600. 
95. Pressure Contour of 3D, Steady-State, Spinning Target in Drift Region at a

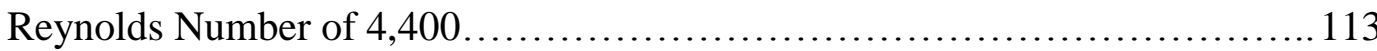

96. Temperature Contour of 3D, Steady-State, Spinning Target in Drift Region at

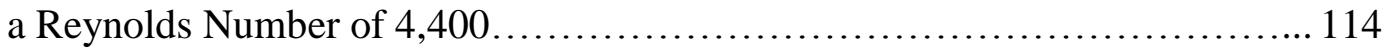

97. Velocity Contour with Streamlines of 3D, Steady-State, Spinning Target in

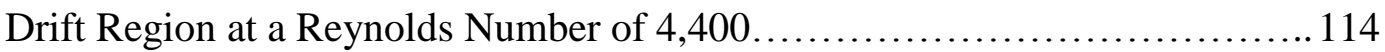

98. Pressure Contour of 3D, Steady-State, Spinning Target in Drift Region at a

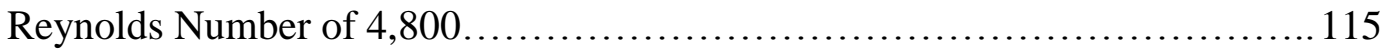

99. Temperature Contour of 3D, Steady-State, Spinning Target in Drift Region at a Reynolds Number of 4,800 .

100. Velocity Contour with Streamlines of 3D, Steady-State, Spinning Target in Drift Region at a Reynolds Number of 4,800 .

101. Pressure Contour of 3D, Steady-State, Spinning Target in Drift Region at a Reynolds Number of 5,200

102. Temperature Contour of 3D, Steady-State, Spinning Target in Drift Region at a Reynolds Number of 5,200.

103. Velocity Contour with Streamlines of 3D, Steady-State, Spinning Target in Drift Region at a Reynolds Number of 5,200....

104. Pressure Contour of 3D, Steady-State, Spinning Target in Drift Region at a Reynolds Number of 5,600

105. Temperature Contour of 3D, Steady-State, Spinning Target in Drift Region at a Reynolds Number of 5,600. 
106. Velocity Contour with Streamlines of 3D, Steady-State, Spinning Target in

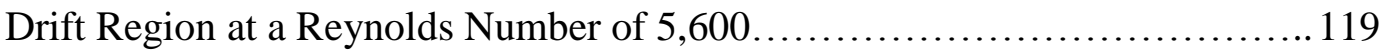

107. Pressure Contour of 3D, Steady-State, Spinning Target in Drift Region with a $1^{\circ}$ Angle of Attack

108. Temperature Contour of 3D, Steady-State, Spinning Target in Drift Region with a $1^{\circ}$ Angle of Attack

109. Pressure Contour of 3D, Steady-State, Spinning Target in Drift Region with a $2^{\circ}$ Angle of Attack

110. Temperature Contour of 3D, Steady-State, Spinning Target in Drift Region with a $2^{\circ}$ Angle of Attack.

111. Pressure Contour of 3D, Steady-State, Spinning Target in Drift Region with a $5^{\circ}$ Angle of Attack

112. Temperature Contour of 3D, Steady-State, Spinning Target in Drift Region with a $5^{\circ}$ Angle of Attack.

113. Pressure Contour of 3D, Steady-State, Spinning Target in Drift Region with a $10^{\circ}$ Angle of Attack

114. Temperature Contour of 3D, Steady-State, Spinning Target in Drift Region with a $10^{\circ}$ Angle of Attack

115. Pressure Contour of 3D, Steady-State, Spinning Target in Drift Region with a $15^{\circ}$ Angle of Attack

116. Temperature Contour of 3D, Steady-State, Spinning Target in Drift Region with a $15^{\circ}$ Angle of Attack. 
117. Pressure Contour of 3D, Steady-State, Spinning Target in Drift Region with a $20^{\circ}$ Angle of Attack

118. Temperature Contour of 3D, Steady-State, Spinning Target in Drift Region with a $20^{\circ}$ Angle of Attack.

119. Drag Force on Spinning Target in Drift Region at Different Angles of Attack from 3D, Steady-State Model.

120. Lift Force on Spinning Target in Drift Region at Different Angles of Attack

from 3D, Steady-State Model.

121.Z-Axis Force on Spinning Target in Drift Region at Different Angles of Attack from 3D, Steady-State Model....

122. Local X-Axis (Axial) Moment on Spinning Target in Drift Region at Different Angles of Attack from 3D, Steady-State Model....

123. Local Y-Axis (negative Magnus) Moment on Spinning Target in Drift Region at Different Angles of Attack from 3D, Steady-State Model.

124.Z-Axis (Negative Overturning) Moment on Spinning Target in Drift Region at Different Angles of Attack from 3D, Steady-State Model 126

125. Drag Force on Non-Spinning Target in Drift Region at Different Angles of Attack from 3D, Steady-State Model.

126. Lift Force on Non-Spinning Target in Drift Region at Different Angles of Attack from 3D, Steady-State Model.

127.Z-Axis Force on Non-Spinning Target in Drift Region at Different Angles of Attack from 3D, Steady-State Model. 128 
128. Local X-Axis (Axial) Moment on Non-Spinning Target in Drift Region at Different Angles of Attack from 3D, Steady-State Model..................... 128

129. Local Y-Axis (Negative Magnus) Moment on Non-Spinning Target in Drift

Region at Different Angles of Attack from 3D, Steady-State Model.............129

130.Z-Axis (Negative Overturning) Moment on Non-Spinning Target in Drift

Region at Different Angles of Attack from 3D, Steady-State Model.............129

131. Angle of Attack over Time for Target with Initial Angle of Attack of $1^{\circ} \ldots \ldots \ldots 131$

132. Angle of Attack over Time for Target with Initial Angle of Attack of $2^{\circ} \ldots \ldots . . .131$

133. Angle of Attack over Time for Target with Initial Angle of Attack of $5^{\circ} \ldots \ldots . .132$

134. Angle of Attack over Time for Target with Initial Angle of Attack of $10^{\circ} \ldots \ldots .132$

135. Angle of Attack over Time for Target with Initial Angle of Attack of $15^{\circ} \ldots \ldots .133$ 


\section{INTRODUCTION}

The National Ignition Facility (NIF) and Laser Inertial Fusion Energy (LIFE)

The National Ignition Facility (NIF) at Lawrence Livermore National Laboratory (LLNL) contains one of the world's largest and most powerful laser systems. The 192 laser beams at the facility can produce almost 2 million joules of energy [6]. One of the main goals of the NIF is to achieve energy gain from thermonuclear fusion of deuterium and tritium [13]. Instead of directly targeting the lasers on the deuterium and tritium, an indirect drive approach has been used [12]. The NIF lasers have been targeted at a hohlraum (gold cylinder surrounding a spherical capsule of deuterium and tritium) [13]. Figure 1 is a picture of one of the targets at the NIF. To achieve thermonuclear fusion burn, the lasers heat the inside of the hohlraum, creating hot plasma that bathes the capsule in X-rays [12]. See Figure 2 for a simulated image of the laser beams heating the hohlraum. The X-rays quickly heat the capsule, which causes an ablation of the outer surface of the capsule [12]. This ablation causes the deuterium and tritium in the capsule to compress to about 100 times the density of solid lead and reach a core temperature over 100 million K [13]. These extreme conditions are required to achieve inertial confinement fusion [13]. The fusion of these 2 hydrogen isotopes can theoretically produce 10 to 100 times more energy than the amount required to power the lasers [13]. 


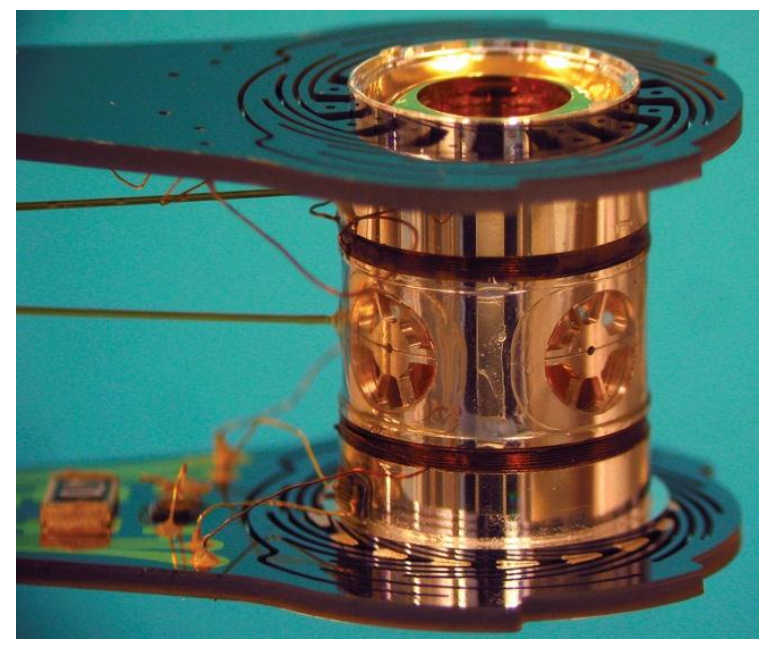

Figure 1. Picture of NIF Target; Credit: Lawrence Livermore National Laboratory [15]

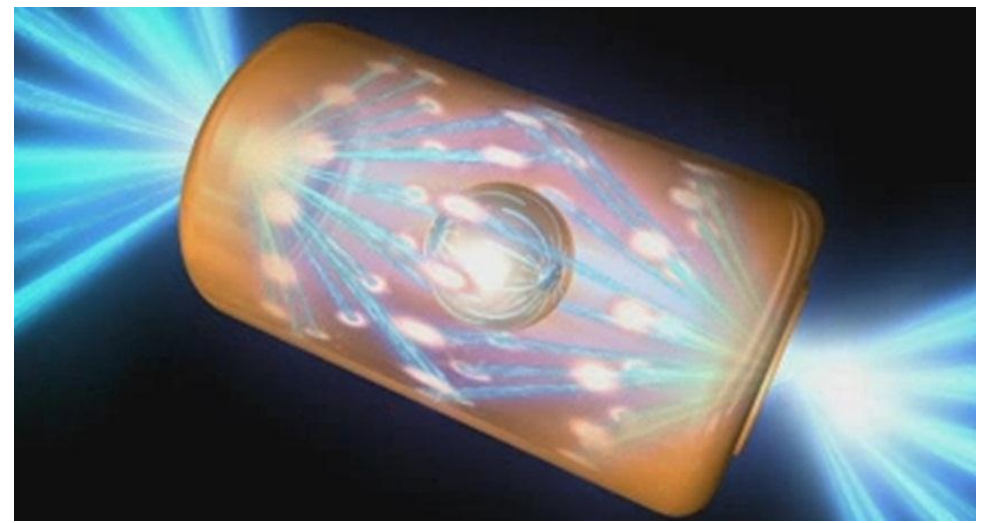

Figure 2. Simulated Image of Lasers Heating NIF Target Hohlraum; Credit: Lawrence Livermore National Laboratory [16]

The Laser Inertial Fusion Energy (LIFE) project was conceived to create a power plant from the fusion technology being developed at the NIF. Energy from the fusion reaction can be used to heat water and generate electricity with steam-turbine generators [6]. The advantages of a LIFE facility include: no harmful emissions, no nuclear waste, and an abundant fuel supply [6]. A LIFE facility would require a target injection system to launch targets into the reaction chamber at approximately $15 \mathrm{~Hz}$ [6]. Note that the LIFE target will be different than the NIF target because of the change from a stationary to a moving target. The first fleet of LIFE plants could each produce $1.5 \mathrm{GW}$ of electricity [6]. The first demonstration plant could be constructed by the late 2020's [6]. 


\section{Target Flight}

The current design for the LIFE target injection requires the target to travel approximately $9 \mathrm{~m}$ through the injector, $4 \mathrm{~m}$ through a drift region ending with a neutron and gamma radiation shielding shutter, and finally $6 \mathrm{~m}$ to the center of the spherical reaction chamber to reach the point of laser contact. See Figure 3 below for the injection path. Note that the injector is directly above the spherical reaction chamber.

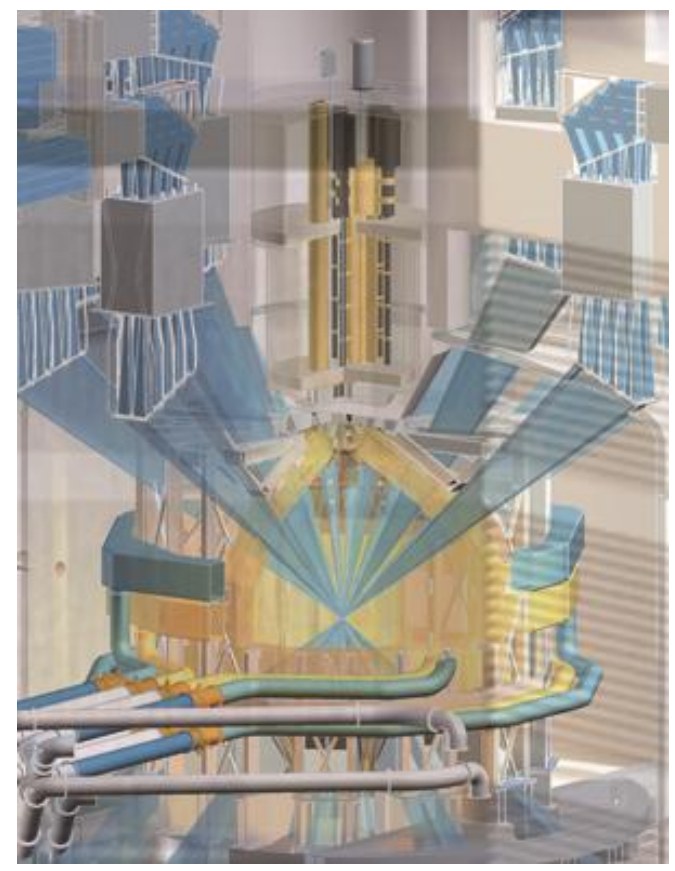

Figure 3. LIFE Reaction Chamber Depicting Lasers and Target Injection Path; Credit: Lawrence Livermore National Laboratory [14]

The target is modeled as a beveled cylinder with an outer diameter of $10.5 \mathrm{~mm}$ and a total length of $13.8 \mathrm{~mm}$. The outer dimensions of the target geometry can be seen in Figure 4. The target is assumed to travel at a constant $250 \mathrm{~m} / \mathrm{s}$, while spinning at 1 revolution per meter of travel, and at a constant temperature of $20 \mathrm{~K}$. The variation in target speed is expected to be no more than $1 \%$ and the heat transfer to the target during this short flight is expected to be minimal, making these modeling assumptions reasonable for this analysis. 


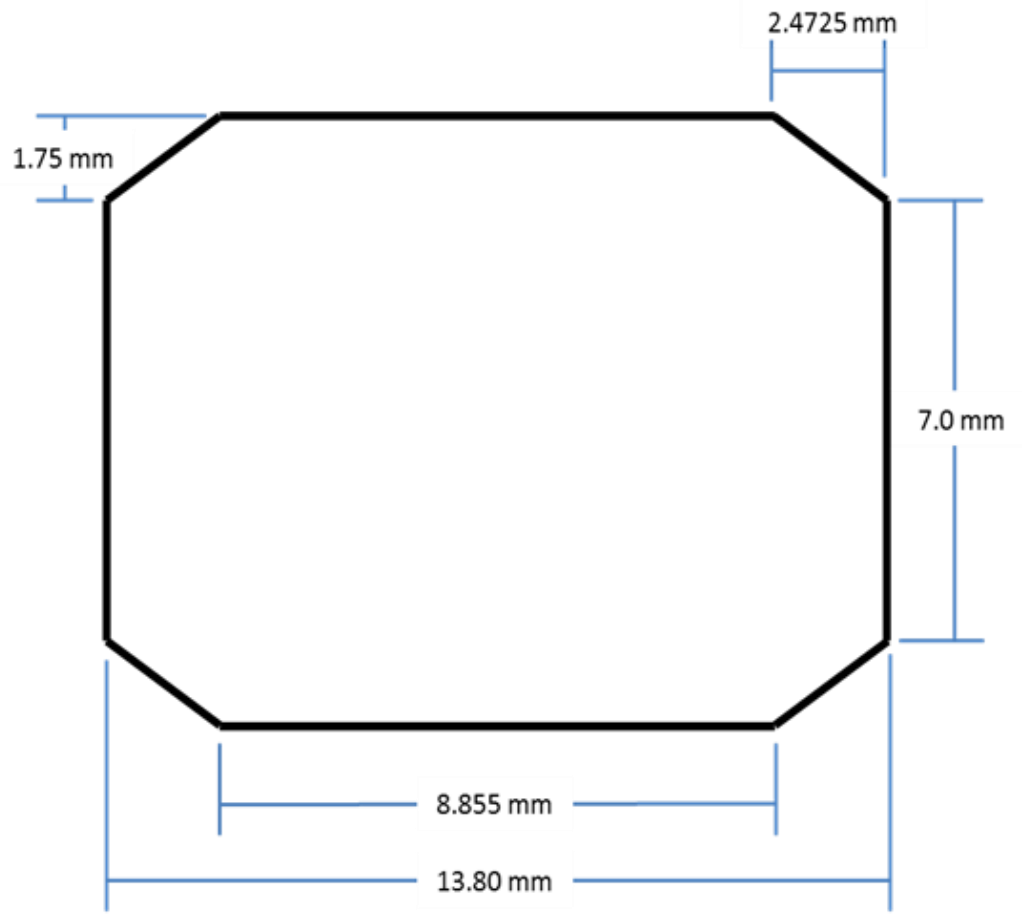

Figure 4. LIFE Target Geometry with Dimensions (Not Drawn to Scale)

In this thesis, the Mach number and Reynolds number are defined as [20],

$$
\begin{gathered}
M a=\frac{V}{c} \\
R e_{d}=\frac{\rho V d}{\mu}
\end{gathered}
$$

where $\mathrm{V}$ is the fluid velocity, $\mathrm{c}$ is the speed of sound, $\rho$ is the fluid density, $\mathrm{d}$ is the characteristic length, and $\mu$ is the dynamic viscosity of the fluid. Note that for external aerodynamic flows, the fluid velocity, $\mathrm{V}$, can also be interpreted as the velocity of a projectile in a stationary fluid. The speed of sound for a perfect gas is defined as [20],

$$
c=\sqrt{\gamma R^{\prime} T}
$$

where $\gamma$ is the ratio of specific heats, $\mathrm{R}^{\prime}$ is the specific gas constant, and $\mathrm{T}$ is the fluid temperature. For monatomic gases like xenon, $\gamma$ is approximately $\frac{5}{3}[19]$.

The $9 \mathrm{~m}$-long injector environment consists of xenon gas at approximately 1,050 K and 3,000 Pa. The Mach number of this regime (with a target speed of $250 \mathrm{~m} / \mathrm{s}$ ) 
is 0.75 and the Reynolds number (using the target's outer diameter as the characteristic length) is 1,768 .

After the injector, the target enters a $4 \mathrm{~m}$-long, cylindrical drift region $0.5 \mathrm{~m}$ in diameter. The region is filled with xenon gas at approximately $1,050 \mathrm{~K}$ and 6,670 $\mathrm{Pa}$. Using the same target speed and characteristic length as before, the Mach number and Reynolds number are 0.75 and 3,937 respectively. The neutron and gamma radiation shielding shutter located at the end of the drift region is a rotating cement disk approximately $0.5 \mathrm{~m}$ in diameter and $1 \mathrm{~m}$ thick. It spins at 4 revolutions per second, and contains a spiral-cut hole $0.2 \mathrm{~m}$ off the axis of rotation for the target to pass through. The target launch will be timed to ensure the target passes through the spiral-cut opening in the shutter.

After the target passes through the shutter, it enters the spherical reaction chamber, which is filled with xenon gas at approximately $6,000 \mathrm{~K}$ and $3,070 \mathrm{~Pa}$. It travels $6 \mathrm{~m}$ to the point of laser contact at the center of the spherical chamber. Using the same target speed and characteristic length as before, the Mach number and Reynolds number in this chamber are 0.31 and 100.5 , respectively. See Table 1 below for a summary of the conditions in each region. Note the extreme combination of low Reynolds numbers and high Mach numbers in the injection and drift regions.

Table 1. Summary of LIFE Target Injection Regimes

\begin{tabular}{|c|c|c|c|c|c|}
\hline Region & $\begin{array}{c}\text { Length of } \\
\text { Flight }(\mathbf{m})\end{array}$ & $\begin{array}{c}\text { Temperature } \\
(\mathbf{K})\end{array}$ & $\begin{array}{c}\text { Pressure } \\
(\mathbf{P a})\end{array}$ & $\begin{array}{c}\text { Mach } \\
\text { Number }\end{array}$ & $\begin{array}{c}\text { Reynolds } \\
\text { Number }\end{array}$ \\
\hline Injection & 9 & 1050 & 3000 & 0.75 & 1768 \\
\hline Drift & 4 & 1050 & 6666 & 0.75 & 3937 \\
\hline Chamber & 6 & 6000 & 3066 & 0.31 & 100.5 \\
\hline
\end{tabular}


The goal of this project was to simulate the target's flight in the drift region using computational fluid dynamics software to highlight key features of the flow, estimate drag and heat transfer as a functions of the Reynolds number, and determine the target's stability. Cases with different target velocities were used to determine Nusselt and drag coefficient correlations. The coefficients of drag, lift, Magnus moment, and overturning moment were used to determine the target's stability. Phase changes of xenon gas were considered negligible since any solidification of xenon is expected to have a minimal effect on the target's flight. Gravity was also neglected. Due to the lack of experimental results for flow over a LIFE target, the results from simulations of flow over a sphere with similar fluid conditions, assumptions, fluid properties, and methods of mesh generation were compared to experimental data and published correlations for validation purposes. Note the sphere simulations are not related to the fuel capsule at the center of the LIFE target. 


\section{List of Terms}

$\mathrm{Ma}=$ Mach number

$\mathrm{V}=$ Velocity in $\mathrm{m} / \mathrm{s}$

$\mathrm{c}=$ Speed of sound or sonic velocity in $\mathrm{m} / \mathrm{s}$

$\mathrm{Re}=$ Reynolds number; the subscript indicates the characteristic length

$\rho=$ Fluid density in $\mathrm{kg} / \mathrm{m}^{3}$

$\mathrm{d}=$ Characteristic length equal to the diameter in $\mathrm{m}$

$\mu=$ Dynamic viscosity in units of Pa-s or $\mathrm{kg} /(\mathrm{m}-\mathrm{s})$

$\gamma=$ Ratio of the specific heat at constant pressure to the specific heat at constant volume

$\mathrm{R}^{\prime}=$ Specific gas constant; ratio of the universal gas constant to the fluid's molecular weight in units of $\mathrm{J} /(\mathrm{kg}-\mathrm{K})$

$\mathrm{T}=$ Temperature in Kelvin

$\varepsilon / \mathrm{k}_{\mathrm{B}}=$ Energy parameter used in kinetic theory of gases.

$\mathrm{k}_{\mathrm{B}}=$ Boltzmann constant equal to $1.381 \mathrm{E}-23 \mathrm{~J} / \mathrm{K}[8]$

$\sigma=$ Characteristic length used in kinetic theory of gases; often reported in angstroms $(\AA)$ where $\AA=10^{-10} \mathrm{~m}$

$\mathrm{b}^{*}=$ First virial coefficient

$\mathrm{B}^{*}=$ Second virial coefficient

$\beta, \Omega^{\prime}{ }_{22}=$ Universal functionals

$\Omega_{22}, \Omega_{11}=$ Collision Integrals

$\mathrm{T}^{*}=$ Non-dimensionalized form of the temperature

$\mathrm{k}=$ Thermal conductivity in units of $\mathrm{W} /(\mathrm{m}-\mathrm{K})$

$\mathrm{M}=$ Molecular weight in $\mathrm{g} / \mathrm{mol}$

$\mathrm{M}^{\prime}=$ Molecular weight in $\mathrm{kg} / \mathrm{mol}$

$\overline{c_{p}}=$ Molar specific heat at constant pressure in $\mathrm{J} /(\mathrm{mol}-\mathrm{K})$

$\mathrm{R}=\mathrm{Universal}$ gas constant equal to $8.3144621 \mathrm{~J} /(\mathrm{mol}-\mathrm{K})$ [22] 
$\mathrm{c}_{\mathrm{p}}=$ Specific heat at constant pressure in $\mathrm{J} /(\mathrm{kg}-\mathrm{K})$

$\mathrm{C}_{\mathrm{D}}=$ Drag coefficient

$\theta_{\mathrm{s}}=$ Separation angle (in degrees) measured from the front stagnation point to the point of separation (in flow over a sphere)

$\mathrm{m}=$ Mass in $\mathrm{kg}$

$\mathrm{S}=$ Reference area in $\mathrm{m}^{2}$

$I_{y}=$ Transverse moment of inertia through an axis through the projectile's center of mass in $\mathrm{kgm}^{2}$

$C_{M_{\alpha}}=$ Overturning moment coefficient

$\mathrm{M}_{\alpha}=$ Overturning moment in $\mathrm{Nm}$

$\alpha_{\mathrm{t}}=$ Total yaw angle in radians

$\mathrm{S}_{\mathrm{d}}=$ Dynamic stability factor

$C_{L_{\alpha}}=$ Lift force coefficient

$C_{M_{p \alpha}}=$ Magnus moment coefficient

$C_{M_{q}}=$ Pitch damping moment coefficient due to transverse angular velocity

$\mathrm{q}_{\mathrm{t}}=$ Transverse angular velocity in $\mathrm{rad} / \mathrm{s}$

$C_{M_{\dot{\alpha}}}=$ Pitch damping moment coefficient due to the angle of attack's rate of change

$\dot{\alpha}_{t}=$ Angle of attack's rate of change in $\mathrm{rad} / \mathrm{s}$

$\mathrm{I}_{\mathrm{x}}=$ Axial moment of inertia in $\mathrm{kgm}^{2}$

$L_{\alpha}=$ Lift force in $\mathrm{N}$

$M_{p \alpha}=$ Magnus moment in $\mathrm{Nm}$

$\mathrm{p}=$ Axial spin in $\mathrm{rad} / \mathrm{s}$

$\mathrm{D}=$ Drag force in $\mathrm{N}$

$M_{p d}=$ Pitch damping moment in Nm

$\mathrm{T}_{0}=$ Total or stagnation temperature in Kelvin 
$\mathrm{St}=$ Strouhal number with characteristic length equal to diameter $\mathrm{d}$

$\mathrm{f}=$ Frequency of oscillation in $\mathrm{Hz}$

$\mathrm{Nu}=$ Nusselt number; the subscript indicates the characteristic length

$\operatorname{Pr}=$ Prandtl number

$\mathrm{x}=\mathrm{axial}$ distance from the front of a cylinder in external flow in $\mathrm{m}$

$\mathrm{L}=$ Chord length of the flat plate strip in $\mathrm{m}$

$\mathrm{a}=$ Acceleration in $\mathrm{m} / \mathrm{s}^{2}$

$\mathrm{s}=$ Displacement in $\mathrm{m}$

$\mathrm{M}_{\mathrm{Z}}=\mathrm{Z}$-axis moment; the negative overturning moment

$\mathrm{A}=$ Angle of attack in degrees 


\section{BACKGROUND}

\section{Xenon Properties}

As mentioned in the target flight section of the introduction, xenon phase changes were ignored. Note that at the drift region pressure of 6,666 Pa, gaseous xenon would undergo deposition at approximately $135 \mathrm{~K}$ according the xenon phase diagram [30]. Knowing that the region at the front stagnation point of the target would see a larger pressure, gaseous xenon could even change to a solid at approximately $150 \mathrm{~K}$. However, the build-up of xenon ice on the target is expected to be thin and blow off quickly. Therefore, xenon property correlations were determined for the gas phase and extended to these lower temperatures as well.

"Most of the better [fluid property] estimation methods use equations based on the form of an incomplete theory with empirical correlations of the constants that are not provided by that theory" [23]. Therefore, the xenon property correlations used in the simulations were a product of an extension of basic kinetic molecular theory with experimentally determined scaling factors. Kestin, Ro, and Wakeham [11] developed equilibrium and transport property correlations for noble gases for a wide range of temperatures and moderate range of pressures.

Kestin et al. [11] used the Chapman-Enskog theory to include the effects of intermolecular forces in the basic kinetic molecular theory. Kestin et al. [11] assumed that xenon, argon, krypton, and neon "obey the same intermolecular force potential" but had different numerical values for the 2 experimentally determined scaling constants: the energy parameter, $\varepsilon / \mathrm{k}_{\mathrm{B}}$, and the characteristic length, $\sigma$. Note that $\mathrm{k}_{\mathrm{B}}$ is the Boltzmann constant; $\varepsilon$ (in units of energy) is the experimentally determined part of the energy parameter. Also, the characteristic length, $\sigma$, is not the same as the characteristic length 
used to define the Reynolds number (d). Viscosity, binary diffusion coefficient, and virial coefficient data over a large temperature range $(60 \mathrm{~K}-2,100 \mathrm{~K})$ was used with universal functions for the first and second virial coefficients $\left(\mathrm{b}^{*}\right.$ and $\left.\mathrm{B}^{*}\right)$, universal functionals $\beta$ and $\Omega_{22}^{\prime}$, and collision integrals $\Omega_{22}$ and $\Omega_{11}$ from Hirschfelder et al. [7] to approximate the parameters [11]. This method provided an energy parameter of 285.27 $\mathrm{K}$ and a characteristic length of $3.858 \AA$ for xenon [11].

With the energy parameter and characteristic length estimated, Kestin et al. [11] used the universal functions Hirschfelder et al. [7] provided to evaluate $\mathrm{B}^{*}, \mathrm{~b}^{*}, \Omega_{22}, \Omega_{11}$, $\Omega_{22}^{\prime}$, and $\beta$ at several temperatures and determine correlations for each in terms of normalized temperature, $\mathrm{T}^{*}$. Kestin et al. [11] estimated the correlation for the collision integral $\Omega_{22}$ as:

$$
\ln \Omega_{22}=0.4394-0.4764\left(\ln T^{*}\right)+0.1235\left(\ln T^{*}\right)^{2}-0.0156\left(\ln T^{*}\right)^{3}
$$

where $\mathrm{T}^{*}$ is the non-dimensionalized form of $\mathrm{T}$ (the temperature in Kelvin) [11]:

$$
T^{*}=\frac{T}{\frac{\varepsilon}{k_{B}}}
$$

Note that correlations depicting the collision integral are typically applicable between $\mathrm{T}^{*}$ of 0.3 and 100 [23].

The temperature, kinetic theory parameters, and collision integral can be used to determine the viscosity and thermal conductivity of the gas. The following equations for dynamic viscosity $(\mu)$ in units of $\mathrm{kg} /(\mathrm{m}-\mathrm{s})$ and thermal conductivity $(\mathrm{k})$ in units of $\mathrm{W} /(\mathrm{m}$ K) have been adapted from Reid et al. [23]:

$$
\begin{gathered}
\mu=(2.669 E-26) \frac{\sqrt{M T}}{\sigma^{2} \Omega_{22}} \\
k=(2.63 E-23) \frac{\sqrt{T / M \prime}}{\sigma^{2} \Omega_{22}}
\end{gathered}
$$


where $\mathrm{M}$ is the molecular weight in $\mathrm{g} / \mathrm{mol}$ and $\mathrm{M}^{\prime}$ is the molecular weight in $\mathrm{kg} / \mathrm{mol}$. According to the Thomas Jefferson National Accelerator Facility [28], the molecular weight of xenon is $131.293 \mathrm{~g} / \mathrm{mol}$.

The preceding equations only account for fluid temperature, not pressure. However, the Chapman-Enskog theory used by Kestin et al. [11] is valid for lowpressure, non-polar gases with only $0.5 \%$ to $1.5 \%$ error [23]. Since the pressure in the drift region is only 6,666 Pa, this theory is adequate in this regime. Additionally, the density of xenon at $1,050 \mathrm{~K}$ and $6,666 \mathrm{~Pa}$ is only $0.10025 \mathrm{~kg} / \mathrm{m}^{3}$; therefore, no highdensity adjustments need to be included for a reasonable approximation of viscosity. Similarly, although thermal conductivity increases as pressure increases, the effect is negligible at low and moderate pressures [23]. Between 100 and 1,000,000 Pa, thermal conductivity only increases about $1 \%$ per $100,000 \mathrm{~Pa}$ [23]. Therefore, the effect of pressure on viscosity and thermal conductivity has been neglected.

Using equations 6 and 7 adapted from Reid et al. [23] with the Kestin et al. [11] kinetic theory parameters and collision integral correlation, the viscosity and thermal conductivity for gaseous xenon were evaluated from $86 \mathrm{~K}$ (the first whole-integer temperature where $\mathrm{T}^{*}$ is above 0.3 ) to $1,050 \mathrm{~K}$ in $1 \mathrm{~K}$ increments. Table 2 shows the viscosity and thermal conductivity of gaseous xenon evaluated at selected temperatures in this region. Best-fit polynomials were determined for the entire set of dynamic viscosity values in units of $\mathrm{kg} /(\mathrm{m}-\mathrm{s})$ and thermal conductivity values in units of $\mathrm{W} /(\mathrm{m}-\mathrm{K})$ :

$$
\begin{gathered}
\mu=(-2.243 E-11) T^{2}+(8.796 E-8) T-1.167 E-6 \\
k=(-5.323 E-9) T^{2}+(2.088 E-5) T-2.769 E-4
\end{gathered}
$$

Note that the $\mathrm{R}^{2}$ value for both correlations was 0.9998 , indicating an excellent curve fit. 
Table 2. Gaseous Xenon Viscosity and Thermal Conductivity at Selected Temperatures Determined by Kinetic Theory

\begin{tabular}{|c|c|c|c|c|}
\hline $\begin{array}{c}\text { Temperature, T } \\
(\mathbf{K})\end{array}$ & $\begin{array}{c}\text { Non-Dimensionalized } \\
\text { Temperature, } \mathbf{T}^{*}\end{array}$ & $\begin{array}{c}\text { Collision } \\
\text { Integral } \mathbf{\Omega}_{\mathbf{2 2}}\end{array}$ & $\begin{array}{c}\text { Viscosity, } \boldsymbol{\mu} \\
(\mathbf{k g} /(\mathbf{m}-\mathbf{s}) \mathbf{)}\end{array}$ & $\begin{array}{c}\text { Thermal Conductivity, } \mathbf{k} \\
(\mathbf{W} / \mathbf{( m - K}) \mathbf{)}\end{array}$ \\
\hline 100 & 0.351 & 2.982 & $6.891 \mathrm{E}-06$ & $1.636 \mathrm{E}-03$ \\
\hline 150 & 0.526 & 2.227 & $1.130 \mathrm{E}-05$ & $2.681 \mathrm{E}-03$ \\
\hline 200 & 0.701 & 1.868 & $1.556 \mathrm{E}-05$ & $3.692 \mathrm{E}-03$ \\
\hline 250 & 0.876 & 1.656 & $1.962 \mathrm{E}-05$ & $4.656 \mathrm{E}-03$ \\
\hline 300 & 1.052 & 1.515 & $2.348 \mathrm{E}-05$ & $5.573 \mathrm{E}-03$ \\
\hline 350 & 1.227 & 1.415 & $2.717 \mathrm{E}-05$ & $6.448 \mathrm{E}-03$ \\
\hline 400 & 1.402 & 1.339 & $3.069 \mathrm{E}-05$ & $7.284 \mathrm{E}-03$ \\
\hline 450 & 1.577 & 1.279 & $3.407 \mathrm{E}-05$ & $8.085 \mathrm{E}-03$ \\
\hline 500 & 1.753 & 1.231 & $3.731 \mathrm{E}-05$ & $8.855 \mathrm{E}-03$ \\
\hline 550 & 1.928 & 1.192 & $4.043 \mathrm{E}-05$ & $9.596 \mathrm{E}-03$ \\
\hline 600 & 2.103 & 1.158 & $4.345 \mathrm{E}-05$ & $1.031 \mathrm{E}-02$ \\
\hline 650 & 2.279 & 1.130 & $4.636 \mathrm{E}-05$ & $1.100 \mathrm{E}-02$ \\
\hline 700 & 2.454 & 1.105 & $4.919 \mathrm{E}-05$ & $1.167 \mathrm{E}-02$ \\
\hline 750 & 2.629 & 1.084 & $5.193 \mathrm{E}-05$ & $1.233 \mathrm{E}-02$ \\
\hline 800 & 2.804 & 1.064 & $5.460 \mathrm{E}-05$ & $1.296 \mathrm{E}-02$ \\
\hline 850 & 2.980 & 1.047 & $5.720 \mathrm{E}-05$ & $1.358 \mathrm{E}-02$ \\
\hline 900 & 3.155 & 1.032 & $5.973 \mathrm{E}-05$ & $1.418 \mathrm{E}-02$ \\
\hline 950 & 3.330 & 1.018 & $6.221 \mathrm{E}-05$ & $1.476 \mathrm{E}-02$ \\
\hline 1000 & 3.505 & 1.005 & $6.463 \mathrm{E}-05$ & $1.534 \mathrm{E}-02$ \\
\hline 1050 & 3.681 & 0.994 & $6.699 \mathrm{E}-05$ & $1.590 \mathrm{E}-02$ \\
\hline & & & & \\
\hline
\end{tabular}

For a monatomic gas like xenon, the specific heat at constant pressure can be approximated with kinetic theory with the following equation from Moran et al. [19]:

$$
\overline{c_{p}}=\frac{5}{2} R
$$

where $\overline{c_{p}}$ is the molar specific heat at constant pressure and $\mathrm{R}$ is the universal gas constant approximated as $8.3144 \mathrm{~J} /(\mathrm{mol}-\mathrm{K})$ [22]. Therefore, for xenon with a molecular weight of $0.131293 \mathrm{~kg} / \mathrm{mol}$ [28], the specific heat at constant pressure $\left(\mathrm{c}_{\mathrm{p}}\right)$ is approximately $158.32 \mathrm{~J} /(\mathrm{kg}-\mathrm{K})$. 


\section{External Flow over a Sphere}

Due to the lack of experimental results for flow over a LIFE target, a similar flow over a sphere was simulated and compared to published results as a form of validation. External flow over a sphere can be categorized into several regimes according to its Reynolds number (where the characteristic length is the diameter of the sphere). Between a Reynolds number of 0 and 20, the flow over a sphere is unseparated, but the streamlines and vorticity contours on the front and back of the sphere are not symmetric due to the fluid's viscosity $[4,20]$. At a Reynolds number of 20, flow separation occurs due to an adverse pressure gradient, which is indicated by a change in the sign of the vorticity [4] as well as zero wall shear stresses and zero velocity gradients at the separation point [20]. Recirculation, or the forming of a wake region behind the sphere, also occurs near a Reynolds number of 20 [4, 21]. Between a Reynolds number of 20 and 130, the flow is steady, axisymmetric about the sphere's centerline (parallel to the free-stream flow), and has an attached wake region $[4,21]$. As the Reynolds number increases, the separation point in each axisymmetric slice moves from the rear stagnation point towards the point where the tangent to the sphere is parallel with the free-stream flow [4]. As the separation point extends along the curve of the sphere from the rear stagnation point, the wake region behind the sphere becomes wider and longer and changes from a concave shape to a convex shape $[4,21]$. The transition point between concave and convex wake shapes occurs near a Reynolds number of 35 [21]. A periodic oscillation of the vortex wake behind the sphere begins between a Reynolds number of 130 and 190 [27, 21]. From a Reynolds number of 210 to 270, the vortices behind the sphere appear as a "two streamwise vortical tails of equal strength and opposite sign" [9]. 
Although the flow is no longer axisymmetric, the vortices are still planar-symmetric [9]. Between a Reynolds of 270 and 400, the flow becomes unsteady, but remains planarsymmetric. Between a Reynolds of 400 and 1000, the flow is no longer planarsymmetric. Above a Reynolds number of 1000, the flow becomes turbulent.

For validation purposes, the 20 to 130 Reynolds range was selected for external flow over a sphere. Since the flow is steady and symmetric in this range, the experimental drag data collected in this range was more reliable than measurements taken during the wake oscillations that occur at higher Reynolds numbers. Additionally, the drag coefficient correlation used for comparison was valid for incompressible flows (at low Mach numbers), which required low velocities and, therefore, low Reynolds numbers [2]. The drag coefficient, separation angle, and wake structure from the Fluent simulations were analyzed within this Reynolds number range and compared to experimental data and published correlations.

A strong relationship between Reynolds number and drag coefficient exists for laminar, incompressible flow over a sphere with wall temperature equal to the freestream temperature [2]. The graph of this relationship is typically referred to as the "standard" drag curve [2]. If the sphere wall temperature is greater than the free-stream temperature, the drag coefficient is greater than would be predicted by the "standard" drag curve [2]. Figure 4 in "Sphere Drag Coefficient for a Broad Range of Mach and Reynolds Numbers" shows the drag coefficient increases with Mach number; however, it appears relatively steady below Mach 0.25 [2]. Clift et al. [4] presented this "standard" drag-Reynolds correlation in the 20 to 260 Reynolds range:

$$
C_{D}=\frac{24}{R e_{d}}\left(1+0.1935 R e_{d}^{0.6305}\right)
$$


Additionally, some of the experimental data from Roos and Willmarth [24] falls within the 20 to 130 Reynolds range. The drag fluctuation during the experiment was estimated to be no more than about $5 \%$ of the average value [24]. This experimental data along with the correlation by Clift et al. [4] served as points of comparison with the drag results from Fluent sphere simulations.

As mentioned previously, the separation point extends from the rear stagnation point along the curve of the sphere as the Reynolds number increases in this range. Clift et al. [4] provided an equation estimating the separation angle (in degrees) measured from the front stagnation point to the point of separation on an axisymmetric slice of the flow as a function of the Reynolds number:

$$
\theta_{S}=180-42.5\left[\ln \frac{R e_{d}}{20}\right]^{0.483}
$$

The separation point coincides with the point of zero shear stress on the wall [20]. The separation angles from the Fluent simulations were determined by identifying the separation point from wall shear stress plots at a variety of Reynolds numbers in this range. The simulation separation angles were compared to the separation angles predicted by Clift's [4] relationship above.

Finally, the wake structure changes as a function of the Reynolds number were observed by Nakamura [21]. As mentioned previously, in the 20-130 Reynolds range, the wake increases with the Reynolds number and the wake changes from concave to convex at approximately a Reynolds number of 35 [21]. The Fluent simulation streamline plots were analyzed to see if this wake structure criterion was met. 


\section{Flight Stability}

Robert McCoy provided stability criteria for a rigid-body, axisymmetric projectile traveling with a small yaw angle along a flat-fire trajectory with negligible wind effects in Modern Exterior Ballistics [17]. Since the flight of the LIFE target satisfies all of these assumptions, McCoy's stability criteria was used to determine the stability of the target in the drift region. The remainder of this flight stability section serves as a summary of McCoy’s flight stability criteria [17].

The variable $M$ was used to differentiate between 2 stability categories: statically stable and statically unstable. $\mathrm{M}$ is defined as:

$$
M=k_{y}{ }^{-2} C_{M_{\alpha}}{ }^{*}
$$

where

$$
\begin{gathered}
k_{y}{ }^{-2}=\frac{m d^{2}}{I_{y}} \\
C_{M_{\alpha}}{ }^{*}=\frac{\rho S d}{2 m} C_{M_{\alpha}}
\end{gathered}
$$

where $\mathrm{m}$ is the projectile mass, $\mathrm{d}$ is the reference diameter of the projectile, $\mathrm{S}$ is the

reference area defined as $S=\frac{\pi d^{2}}{4}, \mathrm{I}_{\mathrm{y}}$ is the transverse moment of inertia on any axis through the projectile's center of mass except the axial centerline, and $C_{M_{\alpha}}$ is the overturning moment coefficient defined by:

$$
M_{\alpha}=\frac{1}{2} \rho V^{2} S d C_{M_{\alpha}} \sin \alpha_{t}
$$

where $\mathrm{M}_{\alpha}$ is the overturning moment associated with the lift or normal force on the projectile, $\mathrm{V}$ is the projectile velocity, and $\alpha_{\mathrm{t}}$ is the total yaw angle.

A statically stable projectile is defined as satisfying $\mathrm{M}<0$. Static stability indicates the projectile is stable without spin. Note that too much spin on a statically 
stable projectile could be destabilizing. If the dynamic stability factor is between 0 and 2 , the statically stable projectile is also dynamically stable. The dynamic stability factor, $S_{d}$, defined as:

$$
S_{d}=\frac{2\left(C_{L_{\alpha}}+k_{x}{ }^{-2} C_{M_{p \alpha}}\right)}{C_{L_{\alpha}}-C_{D}-k_{y}{ }^{-2}\left(C_{M_{q}}+C_{M_{\dot{\alpha}}}\right)}
$$

where $C_{L_{\alpha}}$ is the lift force coefficient, $C_{M_{p \alpha}}$ is the magnus moment coefficient, $C_{M_{q}}$ is the pitch damping moment coefficient due to transverse angular velocity $\left(\mathrm{q}_{\mathrm{t}}\right), C_{M_{\dot{\alpha}}}$ is the pitch damping moment coefficient due to the angle of attack's rate of change $\left(\dot{\alpha}_{t}\right)$, and $k_{x}{ }^{-2}$ is given by

$$
k_{x}^{-2}=\frac{m d^{2}}{I_{x}}
$$

where $I_{x}$ is the axial moment of inertia. The coefficients used in the definition of the dynamic stability factor are determined from the following equations:

$$
\begin{gathered}
L_{\alpha}=\frac{1}{2} \rho V^{2} S C_{L_{\alpha}} \sin \alpha_{t} \\
M_{p \alpha}=\frac{1}{2} \rho V^{2} S d\left(\frac{p d}{V}\right) C_{M_{p \alpha}} \sin \alpha_{t} \\
D=-\frac{1}{2} \rho V^{2} S C_{D} \\
M_{p d}=\frac{1}{2} \rho V^{2} S d\left(\frac{q_{t} d}{V}\right)\left(C_{M_{q}}+C_{M_{\dot{\alpha}}}\right)
\end{gathered}
$$

where $L_{\alpha}$ is the lift force (perpendicular to the projectile's trajectory), $M_{p \alpha}$ is the magnus moment due to the force created by unequal pressures on opposite sides of a spinning projectile, $\mathrm{p}$ is the axial spin in $\mathrm{rad} / \mathrm{s}, \mathrm{D}$ is the drag force (opposing the projectile's motion), and $M_{p d}$ is the pitch damping moment due to the fluid's resistance of the projectile's pitching motion. 
A statically unstable projectile is defined as satisfying $M>0$. Unlike statically stable projectiles, a statically unstable projectile must meet both gyroscopic and dynamic stability criteria to be stable. Gyroscopic stability is defined as satisfying the following:

$$
P^{2}-4 M>0
$$

where $\mathrm{M}$ is defined in equation 13 and $\mathrm{P}$ is defined as:

$$
P=\frac{I_{x}}{I_{y}}\left(\frac{p d}{V}\right)
$$

As with statically stable projectiles, statically unstable projectiles must also satisfy the dynamic stability criteria: $0<S_{d}<2$ where $S_{d}$ is defined in equation 17 . If the statically unstable projectile satisfies the gyroscopic and dynamic stability criteria, it can be spinstabilized. The following inequality can be used to determine a suitable axial spin rate:

$$
P^{2}>\frac{4 M}{S_{d}\left(2-S_{d}\right)}
$$

The aerodynamic coefficients and target stability defined by McCoy were evaluated for the LIFE target at different angles of attack in the drift region. Estimates for the mass and moments of inertia for the target were provided by the LIFE team and can be seen below in Table 3 .

Table 3. Estimates for Target Mass and Moments of Inertia

\begin{tabular}{|c|c|}
\hline Target Mass & $3.033 \mathrm{E}-3 \mathrm{~kg}$ \\
\hline Axial Moment of Inertia, $\mathbf{I}_{\mathbf{x}}$ & $5.72 \mathrm{E}-8 \mathrm{kgm}^{2}$ \\
\hline Transverse Moment of Inertia, $\mathbf{I}_{\mathbf{y}}$ & $8.6 \mathrm{E}-8 \mathrm{kgm}^{2}$ \\
\hline
\end{tabular}




\section{COMPUTATIONAL FLUID DYNAMICS (CFD)}

A collection of programs within ANSYS workbench version 14.5.7 was used to create, run, and post-process all simulations. ANSYS mesh was used to create all meshes (both 2D and 3D). ANSYS Fluent was used to create and run the CFD simulations. ANSYS CFD-Post was used for visual post-processing.

As described in the "ANSYS Fluent User's Guide: Release 14.5” [1], Fluent uses the finite volume method to numerically approximate partial differential equations in fluid dynamic theory (continuity, conservation of momentum, and in the case of compressible flow or heat transfer, conservation of energy) [1]. Fluent provides 2 types of solvers to evaluate these equations. The density-based solver was developed for compressible flows and uses continuity to solve for density and an equation of state (i.e. ideal gas law) to solve for pressure [1]. The pressure-based solver was developed for incompressible flows and calculates pressure with a pressure-correction equation (created from the combination of continuity and conservation of momentum) [1]. Both the density-based and pressure-based solvers calculate velocity with conservation of momentum [1].

This chapter discusses Fluent case setup as well as mesh refinement, domain size, and transient considerations. 


\section{Sphere Validation: Fluent Setup}

As mentioned previously, flow over a sphere was done for validation purposes due to the lack of experimental results for flow over a LIFE target. The drift conditions were applied to the simulations of flow around a sphere. The external flow was xenon at 1,050 $\mathrm{K}$ and 6,666 Pa. The sphere diameter was set at $0.01 \mathrm{~m}$, which is comparable to the outer diameter of the target. As discussed in the sphere background section, the simulations were done within the Reynolds number range of 20-130 to compare with more reliable (steady) drag measurements and to maintain the validity of the incompressible assumption used in the Clift et al. [4] drag coefficient correlation. Additionally, no heat flux was applied at the sphere wall to avoid discrepancy with the Clift et al. [4] drag coefficient correlation.

Since the flow in this regime is steady and axisymmetric, a 2D, steady, axisymmetric model was utilized in the Fluent simulations replicating this flow. The axis was defined as the sphere's centerline (parallel to the free-stream flow). The outer boundary was treated as a wall with no shear and no heat flux. The sphere wall was specified as a no-slip wall with zero heat flux. The inlet was a mass-flow inlet, and the outlet was a pressure outlet. To achieve different Reynolds numbers, the velocity was allowed to change. Velocity and inlet size were used to calculate the flow rate, which was an input for the mass-flow inlet boundary condition. Total (or stagnation) temperature (which was specified at the inlet and outlet) was also a function of velocity and given by [20],

$$
T_{0}=T+\frac{V^{2}}{2 c_{p}}
$$


The Reynolds number, velocity, mass flow rate, total temperature, and Mach number for each case are provided in Table 4. The Reynolds numbers for the cases were selected to match those from the Roos and Willmarth [24] data in the 20-130 Reynolds number range. Note the Mach numbers were below 0.03, which indicates incompressible flow.

Table 4. Input Parameters for Sphere Validation Cases with Different Reynolds Numbers

\begin{tabular}{|c|c|c|c|c|}
\hline $\mathbf{R e}_{\mathbf{d}}$ & Velocity $\mathbf{( \mathbf { m } / \mathbf { s } )}$ & Mass Flow Rate $\mathbf{( k g} / \mathbf{s})$ & Total Temperature $(\mathbf{K})$ & Mach Number \\
\hline 21.1 & 1.4102 & 0.010670 & 1050.01 & 0.00424 \\
\hline 23.4 & 1.5639 & 0.011833 & 1050.01 & 0.00470 \\
\hline 29.1 & 1.9448 & 0.014716 & 1050.01 & 0.00584 \\
\hline 45 & 3.0074 & 0.022756 & 1050.03 & 0.00903 \\
\hline 50.6 & 3.3817 & 0.025588 & 1050.04 & 0.01016 \\
\hline 54.4 & 3.6357 & 0.027510 & 1050.04 & 0.01092 \\
\hline 68.9 & 4.6047 & 0.034842 & 1050.07 & 0.01383 \\
\hline 78.2 & 5.2263 & 0.039545 & 1050.09 & 0.01570 \\
\hline 88.1 & 5.8879 & 0.044552 & 1050.11 & 0.01769 \\
\hline 93.8 & 6.2688 & 0.047434 & 1050.12 & 0.01883 \\
\hline 101 & 6.7500 & 0.051075 & 1050.14 & 0.02028 \\
\hline 104 & 6.9505 & 0.052592 & 1050.15 & 0.02088 \\
\hline 108 & 7.2178 & 0.054615 & 1050.16 & 0.02168 \\
\hline 109 & 7.2847 & 0.055121 & 1050.17 & 0.02188 \\
\hline 124 & 8.2871 & 0.062706 & 1050.22 & 0.02489 \\
\hline 130 & 8.6881 & 0.065740 & 1050.24 & 0.02610 \\
\hline
\end{tabular}

The pressure-based solver was selected due to the incompressibility indicated by the low Mach numbers of the simulations. Due to the high temperature and low pressure in the drift region, the ideal gas assumption was reasonable and used for the density calculations. The coupled solver was selected along with $2^{\text {nd }}$-order upwinding schemes, $2^{\text {nd }}$-order pressure discretization, and Green Gauss node-based gradient discretization for improved accuracy. Since no heat flux was specified, constant properties were used. Viscosity and thermal conductivity were approximated at $1,050 \mathrm{~K}$ from Table 2 in the xenon properties section. See Table 5 for a complete list of properties for the sphere 
simulations. The pseudo transient option was selected to improve the efficiency of the calculations. Default settings for the relaxation factors and solution limits were used. The solution was initialized from the inlet conditions. See Table 22 in Appendix A for a detailed list of the setup. All simulations discussed in the sphere validation sections were run on a single processor on a Windows PC, and all resulting residuals were less than 1E9.

Table 5. Xenon Properties for Sphere Validation Simulations in Drift Region at 1,050 K and 6,666 Pa

\begin{tabular}{|c|c|}
\hline Properties & Value \\
\hline Dynamic Viscosity, $\mu$ & $6.70 \mathrm{E}-5 \mathrm{~kg} /(\mathrm{m}-\mathrm{s})$ \\
\hline Thermal Conductivity, $\mathrm{k}$ & $0.0159 \mathrm{~W} /(\mathrm{m}-\mathrm{K})$ \\
\hline Specific Heat at Constant Pressure, $\mathrm{c}_{\mathrm{p}}$ & $158.32 \mathrm{~J} /(\mathrm{kg}-\mathrm{K})$ \\
\hline Molecular Weight, $M$ & $131.293 \mathrm{~g} / \mathrm{mol}$ \\
\hline
\end{tabular}

Before running the Fluent simulations at the different Reynolds numbers, the size of the domain and mesh refinement were studied. These were analyzed for the setup at a Reynolds number of 101 (with a velocity of $6.75 \mathrm{~m} / \mathrm{s}$ ). 


\section{Sphere Validation: Domain Size and Mesh Refinement Studies}

The first domain considered consisted of 10 body lengths before the sphere, 15 body lengths above the sphere, and 20 body lengths behind the sphere (where the body length is the sphere's diameter). Each mesh was made with a 1.02 overall growth rate (which has been determined to be sufficiently refined in the mesh refinement section), 12 inflation layers surrounding the sphere wall with a 1.10 growth rate and a default transition ratio of 0.272 , edge sizing along the sphere wall of $1 \mathrm{E}-4 \mathrm{~m}$, and a triangledominated meshing method. All simulations had the same Fluent case setup with a Reynolds number of 101. See Table 6 and Figure 5 for the drag results from the different domain sizes.

Table 6. Sphere Domain Size Study

\begin{tabular}{|c|c|c|c|c|c|c|}
\hline Case & $\begin{array}{c}\text { Body Lengths } \\
\text { Before }\end{array}$ & $\begin{array}{c}\text { Body Lengths } \\
\text { Above }\end{array}$ & $\begin{array}{c}\text { Body Lengths } \\
\text { Behind }\end{array}$ & Nodes & $\begin{array}{c}\text { Drag } \\
\text { Force (N) }\end{array}$ & $\begin{array}{c}\text { Drag } \\
\text { Coefficient }\end{array}$ \\
\hline 1 & 10 & 15 & 20 & 28496 & $1.9480 \mathrm{E}-04$ & 1.08597 \\
\hline 2 & 5 & 15 & 20 & 26616 & $1.9581 \mathrm{E}-04$ & 1.09161 \\
\hline 3 & 20 & 15 & 20 & 29391 & $1.9457 \mathrm{E}-04$ & 1.08473 \\
\hline 4 & 30 & 15 & 20 & 29944 & $1.9455 \mathrm{E}-04$ & 1.08460 \\
\hline 5 & 10 & 7 & 20 & 24334 & $1.9511 \mathrm{E}-04$ & 1.08770 \\
\hline 6 & 10 & 25 & 20 & 29680 & $1.9475 \mathrm{E}-04$ & 1.08569 \\
\hline 7 & 10 & 15 & 5 & 25652 & $1.9474 \mathrm{E}-04$ & 1.08566 \\
\hline 8 & 10 & 15 & 10 & 26642 & $1.9475 \mathrm{E}-04$ & 1.08569 \\
\hline 9 & 10 & 15 & 30 & 28738 & $1.9475 \mathrm{E}-04$ & 1.08570 \\
\hline 10 & 20 & 15 & 5 & 26962 & $1.9455 \mathrm{E}-04$ & 1.08462 \\
\hline
\end{tabular}




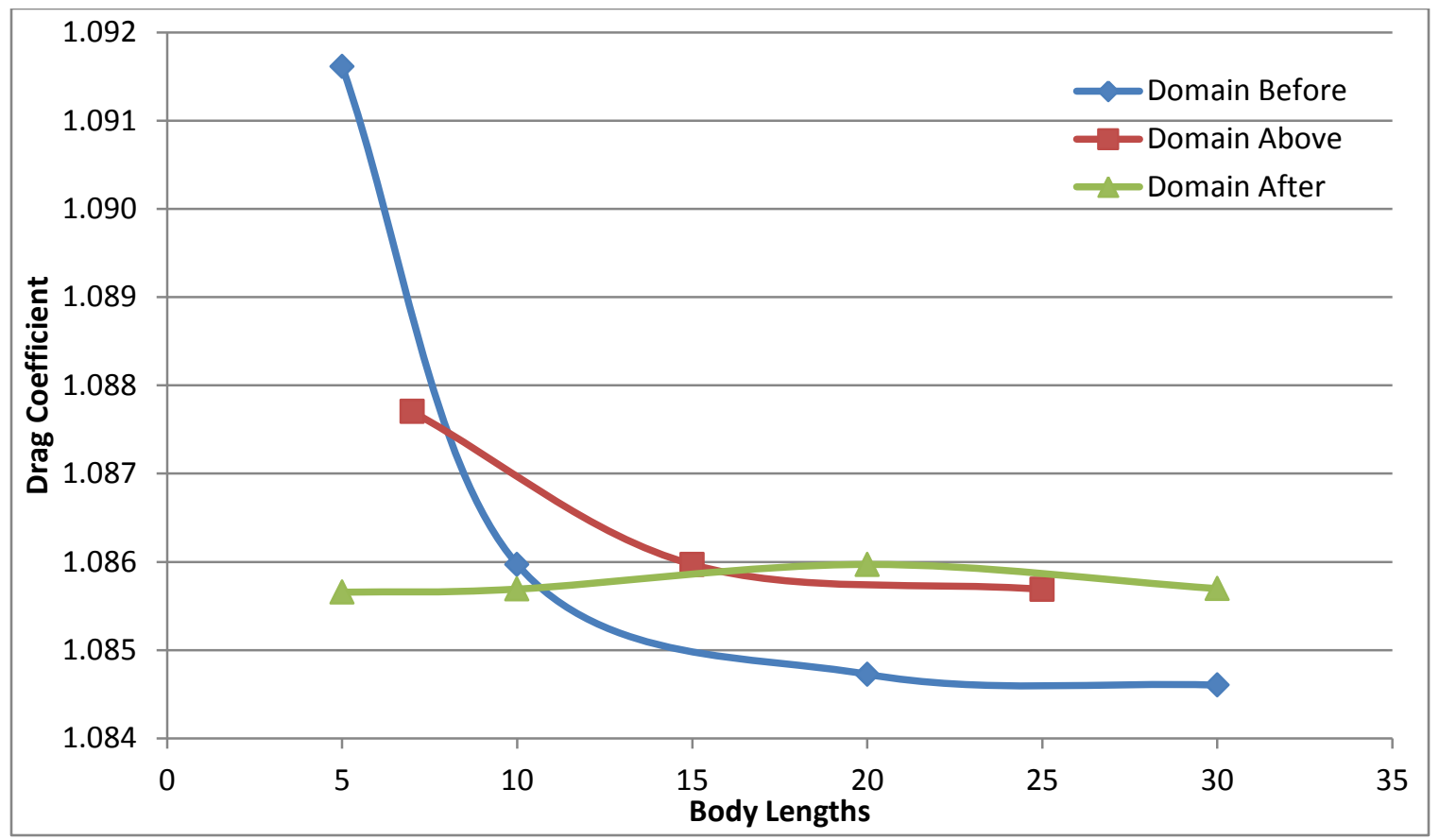

Figure 5. Sphere Domain Size Study at $\mathrm{Re}=101$

As can be seen in the mesh refinement section, the converged drag coefficient value appeared to be close to 1.084 . Therefore, cases 2 (with only 5 body lengths before the sphere) and 5 (with only 7 body lengths above the sphere) seemed to produce the greatest error. Typically, as the domain increased in any direction, the drag coefficient decreased towards 1.084. When analyzing the distance before the sphere, there was only a $0.012 \%$ difference in the drag coefficient between the cases with 30 body lengths before and 20 body lengths before. The case with 10 body lengths before had 10 times this percent difference from the case with 30 body lengths before. The case with 5 body lengths before was even worse with 50 times the percent difference. Therefore, 20 body lengths before the sphere appeared adequate to model the flow properly. When analyzing the domain above the sphere, cases with 25 and 15 body lengths above the sphere had only a $0.026 \%$ difference in drag. Cases with 25 and 7 body lengths above the sphere had over 7 times this percent difference. Therefore, a distance of 15 body lengths was 
chosen as the size of the domain above the sphere to sufficiently capture the flow. Very little percent difference in drag occurred between the cases with different domain sizes behind the sphere. Therefore, the smallest domain size simulated behind the sphere appeared acceptable. However, as the distance behind the sphere decreases, the domain could encroach upon the wake region, thus inaccurately predicting the drag coefficient. As a check, Taneda's [27] wake length plot was used to approximate the maximum wake length in this Reynolds number range. Taneda's wake length plot predicted an upper wake-length limit of 1.5 body lengths [27]. Therefore, including 5 body lengths behind the sphere would safely avoid inaccuracies due to encroachment on the wake. Based on these results, 20 body lengths before, 15 body lengths above, and 5 body lengths behind the sphere seemed to provide the most computationally efficient and accurate results. The drag results with the final domain selected can be seen in case 9 . Note that the drag coefficient from case 9 was almost the same as the drag coefficient closest to the converged value of 1.084 in Table 6 (found in case 4). Note that case 4 had one of the largest domain sizes and the most nodes. Compared to case 4, case 9 provided almost the same drag coefficient with almost 3,000 less nodes.

The mesh refinement study has been done with the chosen domain size: 20 body lengths before, 15 body lengths above, and 5 body lengths behind the sphere. As in the domain size study, 12 inflation layers with a 1.10 growth rate and default transition ratio of 0.272 were used around the sphere wall to accurately resolve the boundary layer.

The results from the mesh refinement study can be seen in Table 7. The first 7 cases were made by decreasing the overall growth rate while keeping the edge size at $1 \mathrm{E}$ $4 \mathrm{~m}$ along the sphere wall. A plot of the drag coefficient from these first 7 meshes can be 
seen in Figure 6. Based on the exceedingly low percent difference between the 1.01 and 1.02 growth rate cases $(0.006 \%)$ with such a large difference in the number of nodes, the 1.02 growth rate case has sufficient refinement. The last 2 cases in Table 7 explored changing the sphere edge size. Note that changing the edge size by a factor of 10 while keeping the overall growth rate at 1.02 had a minimal effect on the results despite a large change in the number of nodes. This minimal drag coefficient change with changing edge size can also be seen in Figure 6. Therefore, the mesh from case 6 with the 1.02 overall growth rate, $1 \mathrm{E}-4 \mathrm{~m}$ edge size around the sphere, and 12 inflation layers with 1.10 growth rate and default transition ratio of 0.272 provided adequate refinement for these simulations. See Figures 7 and 8 for images of the selected mesh.

Table 7. Sphere Mesh Refinement Study

\begin{tabular}{|c|c|c|c|c|c|}
\hline \multirow[b]{2}{*}{ Case } & \multicolumn{2}{|c|}{ Mesh Type } & \multirow{2}{*}{ Nodes } & \multirow{2}{*}{$\begin{array}{l}\text { Drag Force } \\
\text { (N) }\end{array}$} & \multirow{2}{*}{$\begin{array}{c}\text { Drag } \\
\text { Coefficient }\end{array}$} \\
\hline & Edge Size (m) & Overall Growth Rate & & & \\
\hline 1 & $1.0 \mathrm{E}-04$ & 1.20 & 5158 & $1.98048 \mathrm{E}-04$ & 1.10410 \\
\hline 2 & $1.0 \mathrm{E}-04$ & 1.15 & 5562 & $1.95884 \mathrm{E}-04$ & 1.09204 \\
\hline 3 & $1.0 \mathrm{E}-04$ & 1.10 & 6423 & $1.95267 \mathrm{E}-04$ & 1.08860 \\
\hline 4 & $1.0 \mathrm{E}-04$ & 1.05 & 9867 & $1.94745 \mathrm{E}-04$ & 1.08569 \\
\hline 5 & $1.0 \mathrm{E}-04$ & 1.03 & 16271 & $1.94627 \mathrm{E}-04$ & 1.08503 \\
\hline 6 & $1.0 \mathrm{E}-04$ & 1.02 & 26962 & $1.94554 \mathrm{E}-04$ & 1.08462 \\
\hline 7 & $1.0 \mathrm{E}-04$ & 1.01 & 66847 & 1.94542E-04 & 1.08456 \\
\hline 8 & $5.0 \mathrm{E}-05$ & 1.02 & 40973 & $1.94548 \mathrm{E}-04$ & 1.08459 \\
\hline 9 & $1.0 \mathrm{E}-05$ & 1.02 & 65660 & $1.94520 \mathrm{E}-04$ & 1.08443 \\
\hline
\end{tabular}




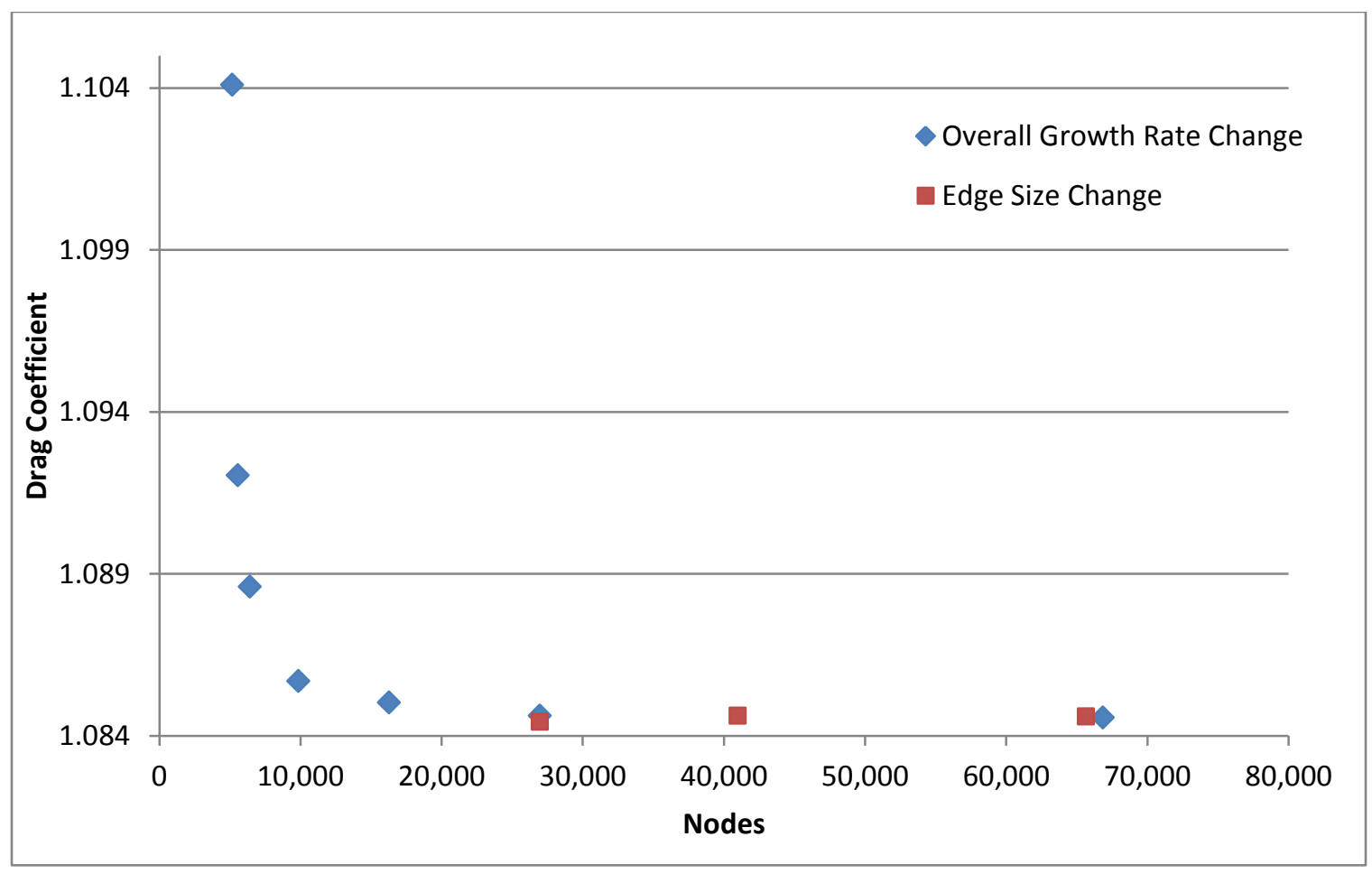

Figure 6. Sphere Mesh Convergence Plot at $\mathrm{Re}=101$

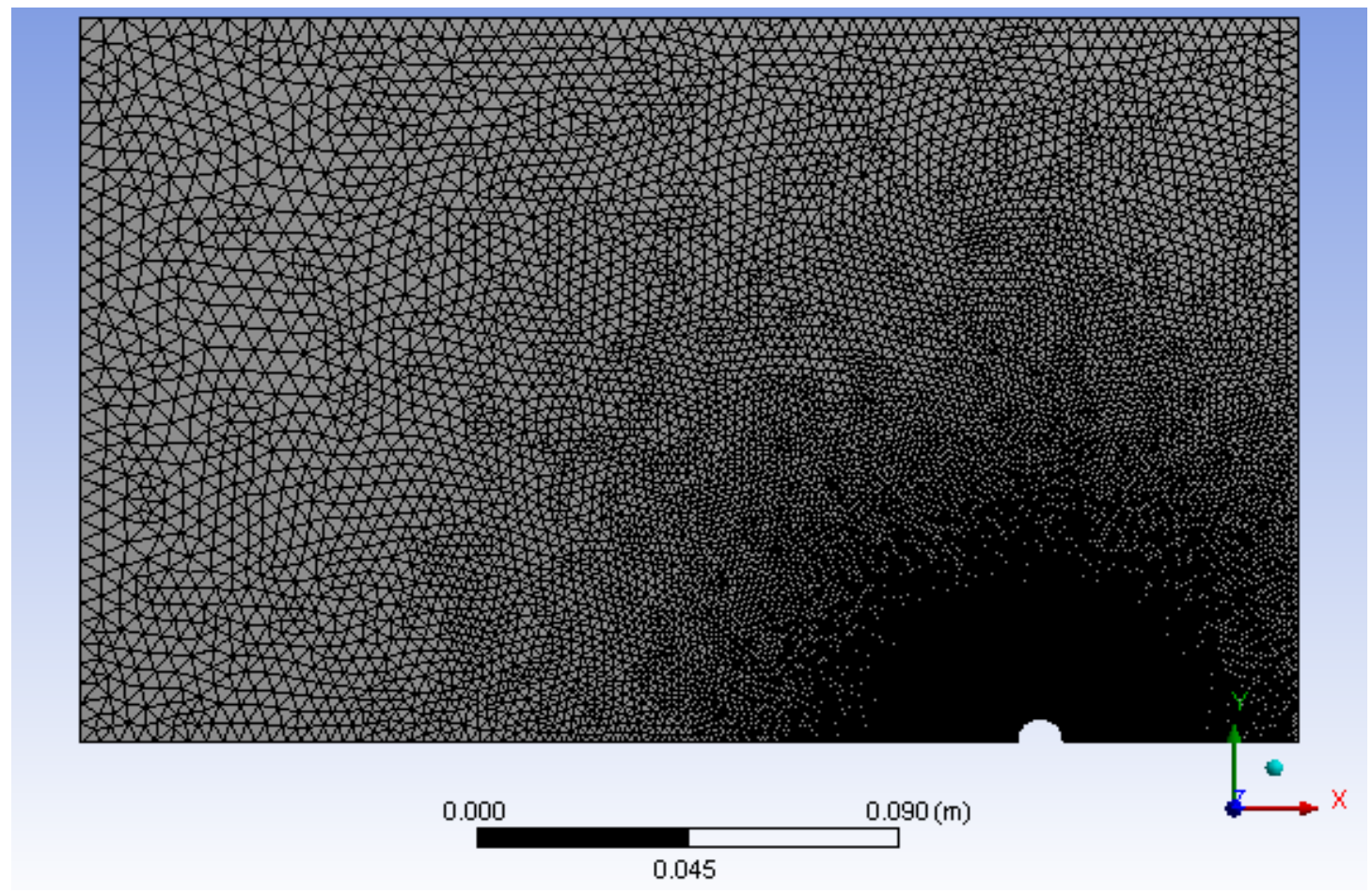

Figure 7. Selected Mesh (Case 6 from Refinement Table) for Sphere Simulations 


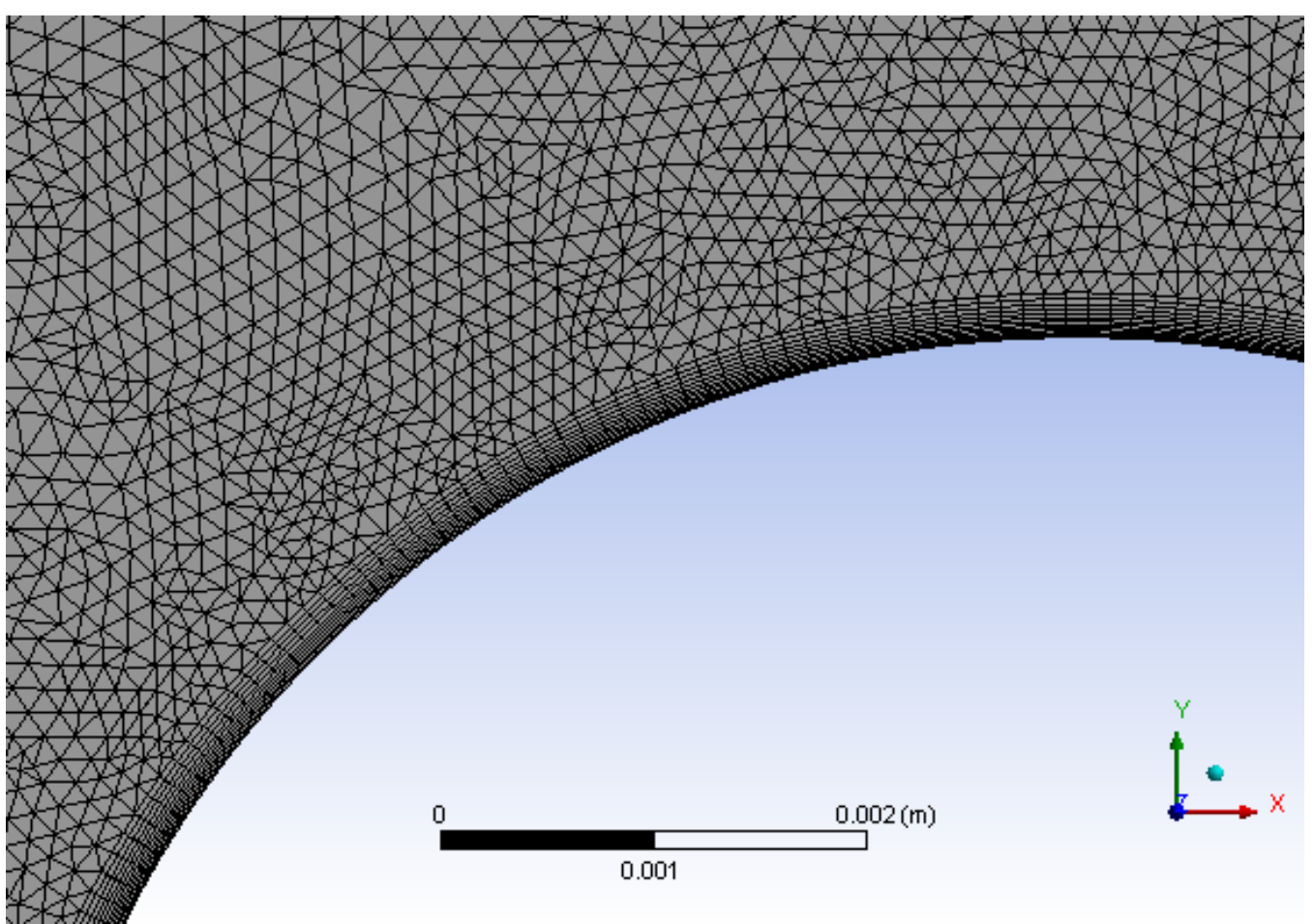

Figure 8. Inflation Layers of Selected Mesh (Case 6 from Refinement Table) for Sphere Simulations 


\section{D, Axisymmetric Drift Regime: Fluent Setup}

As mentioned in the introduction, the drift region consists of xenon gas at $1,050 \mathrm{~K}$ and 6,666 Pa. The target has a forward velocity of $250 \mathrm{~m} / \mathrm{s}$, spin of 1 revolution per meter traveled, and wall temperature of $20 \mathrm{~K}$. A 2D, axisymmetric swirl model of this flow was utilized first. A 3D model was also done (see sections pertaining to the 3D drift regime) to determine if the flow around the target could be adequately simulated with a 2D, axisymmetric model and to evaluate the target's flight stability. The remainder of this section discusses the setup for the 2D, axisymmetric swirl model.

The axis of symmetry was the target's centerline (parallel to the free-stream flow). A far-field pressure boundary condition was specified for the inlet with a Mach number of approximately 0.75 in the axial direction and a static temperature $1,050 \mathrm{~K}$. The outlet was a pressure outlet with a backflow total temperature of approximately $1,250 \mathrm{~K}$. (The total temperature was calculated with equation 26 from the sphere Fluent setup section.) The outer boundary of the domain was treated as a wall with no shear and no heat flux. Each target wall was specified with no slip and given an absolute rotational speed of approximately $1,571 \mathrm{rad} / \mathrm{s}$ and a wall temperature of $20 \mathrm{~K}$. The operating pressure of the flow was approximately 6,666 Pa.

Since the high Mach number in the drift region indicated a compressible flow, the density-based solver was selected over the pressure-based solver. The density was treated as an ideal gas due to the high temperature and low pressure in the drift region. The ANSYS Fluent user guide generally recommended the implicit solver and Roe-FDS convective flux for use with the density-based solver [1]. Therefore, they were selected for these simulations. Green Gauss node-based gradient discretization was used for improved accuracy, but only $1^{\text {st }}$-order upwinding schemes for flow, turbulent kinetic 
energy, and specific dissipation rate were used to ensure solution convergence. Due to the varying temperature from the wall to the free-stream, property correlations from equations 8 and 9 were used from the xenon properties section. See Table 8 for a summary of the xenon properties.

Table 8. Xenon Properties in Drift Region for Target Simulations

\begin{tabular}{|c|c|c|}
\hline Properties & Value & Units \\
\hline Dynamic Viscosity, $\mu$ & $(-2.243 \mathrm{E}-11) \mathrm{T}^{2}+(8.796 \mathrm{E}-8) \mathrm{T}-1.167 \mathrm{E}-6$ & $\mathrm{~kg} /(\mathrm{m}-\mathrm{s})$ \\
\hline Thermal Conductivity, $\mathrm{k}$ & $(-5.323 \mathrm{E}-9) \mathrm{T}^{2}+(2.088 \mathrm{E}-5) \mathrm{T}-2.769 \mathrm{E}-4$ & $\mathrm{~W} /(\mathrm{m}-\mathrm{K})$ \\
\hline Specific Heat at Constant Pressure, $\mathrm{c}_{\mathrm{p}}$ & 158.32 & $\mathrm{~J} /(\mathrm{kg}-\mathrm{K})$ \\
\hline Molecular Weight, $M$ & 131.293 & $\mathrm{~g} / \mathrm{mol}$ \\
\hline
\end{tabular}

As mentioned in the introduction, the Reynolds number in the drift region is 3,937. For pipe flows, the transition between laminar and turbulent flow occurs between a Reynolds number of 2,100 and 4,000 (where the characteristic length is the pipe diameter) [20]. However, this transition is more difficult to identify for external flows due to the complexity introduced by object curvature [20], the somewhat arbitrary selection of the characteristic length, and other parameters. Typically, the transition from laminar to turbulent for external flow occurs with a Reynolds number on the order of $10^{5}$ [20]. However, the only way to accurately determine whether the external flow is laminar or turbulent is through physical observation. Assuming the flow was laminar, several 2D, axisymmetric swirl, laminar target simulations were created and run. However, after performing domain size, mesh refinement, and transient studies, a converged solution could not be obtained. Therefore, a weak turbulence model was implemented instead. Although the k-epsilon models are the most widely used turbulence models, they tend to not fully account for the adverse pressure gradients and 
predict delayed or reduced boundary layer separation [1]. The k-omega SST model was designed to "accurately compute flow separation from smooth surfaces" and has become the "most widely used...for aerodynamic flows" [1]. Therefore, the k-omega SST turbulence model with a weak turbulent intensity of $1 \%$ and a small turbulent viscosity ratio of 1 at the inlet (and for any backflow at the outlet) was used in each simulation presented in the drift regime sections.

A converged, steady-state solution for the 2D, axisymmetric swirl model was not obtainable for the spinning target in the drift region. Therefore, a transient model was employed. See the following section for details on how the time step size was selected. The time step used was 5.0E-7 s. Absolute convergence criteria set for all residuals was 1E-8. The maximum number of iterations per time step was 150 . The residuals at the end of each time step never exceeded $10^{-3}$, which is a popular absolute convergence limit mentioned in the ANSYS Fluent user guide [1]. The transient formulation was $1^{\text {st }}$-order implicit.

The default courant number and under-relaxation factors were used. The solution was initialized from the inlet conditions. The energy equation was automatically turned on. See these and other specific input parameters in Table 23 in Appendix A. 


\section{D, Axisymmetric Drift Regime: Domain Size, Mesh Refinement, and Transient}

\section{Considerations}

For the sphere validation section, several cases were run to determine not only a converged solution, but also an optimal and computationally efficient case. Since these cases were steady-state simulations, these additional runs took less than 10 minutes. As explained in the previous section, simulating the spinning target in the drift region required transient simulations. Since transient cases must calculate a solution at each time step instead of one solution at steady state, transient simulations take much longer to complete. On a single processor, these simulations with 2,000 time steps typically required 2-3 days. (The exact runtime varied depending on the number of nodes in each simulation.) Therefore, simple convergence studies were performed to identify a 2D, axisymmetric target case that produced a reasonably converged solution. Computational efficiency was not explored.

An initial guess was made for adequate domain size and mesh refinement for the 2D target simulations based on the domain size and mesh refinement studies with the sphere. The initial target domain extended 20 body lengths in front of the target, 15 body lengths above the target, and 20 body lengths behind the target. (Note the target body length is equal to $13.8 \mathrm{~mm}$.) Although a distance of only 5 body lengths behind the sphere was acceptable in the sphere simulations, the target domain was extended due to the larger Reynolds number of the flow around the target. Like the sphere mesh, the mesh of the target domain was given 12 inflation layers with a growth rate of 1.10 and default transition ratio of 0.272 , an overall growth rate of 1.02 , and a triangle-dominated meshing method. However, the edge size along the projectile wall was reduced from 1E$4 \mathrm{~m}$ to $5 \mathrm{E}-5 \mathrm{~m}$ due to the angularity of the target's geometry. 
An initial guess for a suitable time step was also based on results from a sphere. Sakamoto and Haniu [25] measured the frequencies of wake fluctuation behind a sphere and plotted the non-dimensional form of this frequency (low-mode Strouhal number) as a function of Reynolds number in the Reynolds number range of 3,000-40,000. All Strouhal numbers $(\mathrm{St})$ in this range were less than 0.26. Using the definition of the Strouhal number [25],

$$
S t=\frac{f d}{V}
$$

the maximum frequency of wake fluctuation (f) for a sphere with the same diameter as the target's outer diameter is approximately 6,190 Hz. Therefore, an oscillation of the wake behind a sphere could occur every 1.6E-4 s. Dividing this period into 150 time steps should allow Fluent to effectively capture the oscillation. This would correspond to a time step of approximately 1.0E-6 s. Since this is an approximation for a sphere, the initial time step used in the drift target simulations was reduced to half of this value or 5.0E-7 s. Note that an oscillating wake behind the target is not guaranteed. The time step was selected to capture a fluctuation if present.

This initial case (with domain, mesh refinement, and time step described previously) was run and compared to cases with larger domains in each direction to determine if the domain of the initial test case ( 20 body lengths before, 15 body lengths above, and 20 body lengths behind) was adequate. The drag coefficient and heat transfer after $1 \mathrm{~ms}$ were used as points of comparison. The results can be seen in Table 9. Note that this is a transient flow, and some of the variance between cases is due to its dynamic nature. Less than a $3.5 \%$ difference in drag and a $2 \%$ difference in heat transfer between cases for a 2D, axisymmetric swirl model of a 3D flow after only $1 \mathrm{~ms}$ is reasonable. 
Therefore the initial domain of 20 body lengths before, 15 body lengths above, and 20 body lengths behind sufficiently encompasses the flow field.

Table 9. Domain Study on 2D, Axisymmetric Swirl, Transient Drift Flow over Target

\begin{tabular}{|c|c|c|c|c|c|c|c|c|c|}
\hline Case & $\begin{array}{c}\text { Body } \\
\text { Lengths } \\
\text { Before }\end{array}$ & $\begin{array}{c}\text { Body } \\
\text { Lengths } \\
\text { Above }\end{array}$ & $\begin{array}{c}\text { Body } \\
\text { Lengths } \\
\text { Behind }\end{array}$ & Nodes & $\begin{array}{c}\text { Drag } \\
\text { Force } \\
\text { (N) at 1 } \\
\text { ms }\end{array}$ & $\begin{array}{c}\text { Drag } \\
\text { Coefficient } \\
\text { at 1 ms }\end{array}$ & $\begin{array}{c}\text { Drag \% } \\
\text { Difference } \\
\text { with Case } \\
\mathbf{1}\end{array}$ & $\begin{array}{c}\text { Heat } \\
\text { Transfer } \\
\text { (W) at 1 } \\
\text { ms }\end{array}$ & $\begin{array}{c}\text { Transfer } \\
\text { \% } \\
\text { Difference } \\
\text { with Case } \\
\mathbf{1}\end{array}$ \\
\hline 1 & 20 & 15 & 20 & 47285 & 0.1512 & 0.5572 & NA & 25.13 & NA \\
\hline 2 & 30 & 15 & 20 & 48232 & 0.1559 & 0.5746 & -3.036 & 25.45 & 1.254 \\
\hline 3 & 20 & 25 & 20 & 49607 & 0.1514 & 0.5580 & -0.149 & 25.44 & 1.223 \\
\hline 4 & 20 & 15 & 30 & 48190 & 0.1533 & 0.5653 & -1.428 & 25.58 & 1.775 \\
\hline
\end{tabular}

The initial case's mesh was then compared to more refined and coarser meshes to determine suitable refinement of the domain. As previously mentioned, the initial test case's mesh had 12 inflation layers with a 1.10 growth rate and a default transition ratio of 0.272 , an overall growth rate of 1.02 , a triangle-dominated meshing method, and a target edge size of $5 \mathrm{E}-5 \mathrm{~m}$. The more refined mesh was made by reducing the edge size to $2.5 \mathrm{E}-5 \mathrm{~m}$ and lowering the overall growth rate to 1.015 . One of the coarser meshes had an increased edge size of 1E-4 m and overall growth rate of 1.06. The coarsest mesh had an edge size of 2.5E-4 m and overall growth rate of 1.08. Table 10 and Figure 9 show the comparison between these 4 levels of refinement based on the drag coefficient and heat transfer after $1 \mathrm{~ms}$. Note that almost doubling the nodes in the mesh from the initial case results in only a $0.75 \%$ difference in drag and only a $-0.04 \%$ difference in heat transfer. Therefore, the initial case is sufficiently refined. Figures 10 and 11 show the final domain and mesh used for the 2D, axisymmetric swirl target simulations. 
Table 10. Mesh Refinement Study on 2D, Axisymmetric Swirl, Transient Drift Flow over Target

\begin{tabular}{|c|c|c|c|c|c|c|}
\hline Case & Nodes & $\begin{array}{c}\text { Drag } \\
\text { Force (N) } \\
\text { at 1 ms }\end{array}$ & $\begin{array}{c}\text { Drag } \\
\text { Coefficient } \\
\text { at 1 ms }\end{array}$ & $\begin{array}{c}\text { Drag \% } \\
\text { Difference } \\
\text { with Case 1 }\end{array}$ & $\begin{array}{c}\text { Heat } \\
\text { Transfer } \\
\text { (W) at 1 ms }\end{array}$ & $\begin{array}{c}\text { Heat } \\
\text { Transfer \% } \\
\text { Difference } \\
\text { with Case1 }\end{array}$ \\
\hline 1 & 47285 & 0.1512 & 0.5572 & NA & 25.13 & NA \\
\hline 2 & 80114 & 0.1523 & 0.5614 & 0.753 & 25.12 & -0.0426 \\
\hline 3 & 10312 & 0.1585 & 0.5841 & 4.830 & 25.17 & 0.1423 \\
\hline 4 & 4851 & 0.1734 & 0.6391 & 14.58 & 28.87 & 14.86 \\
\hline
\end{tabular}

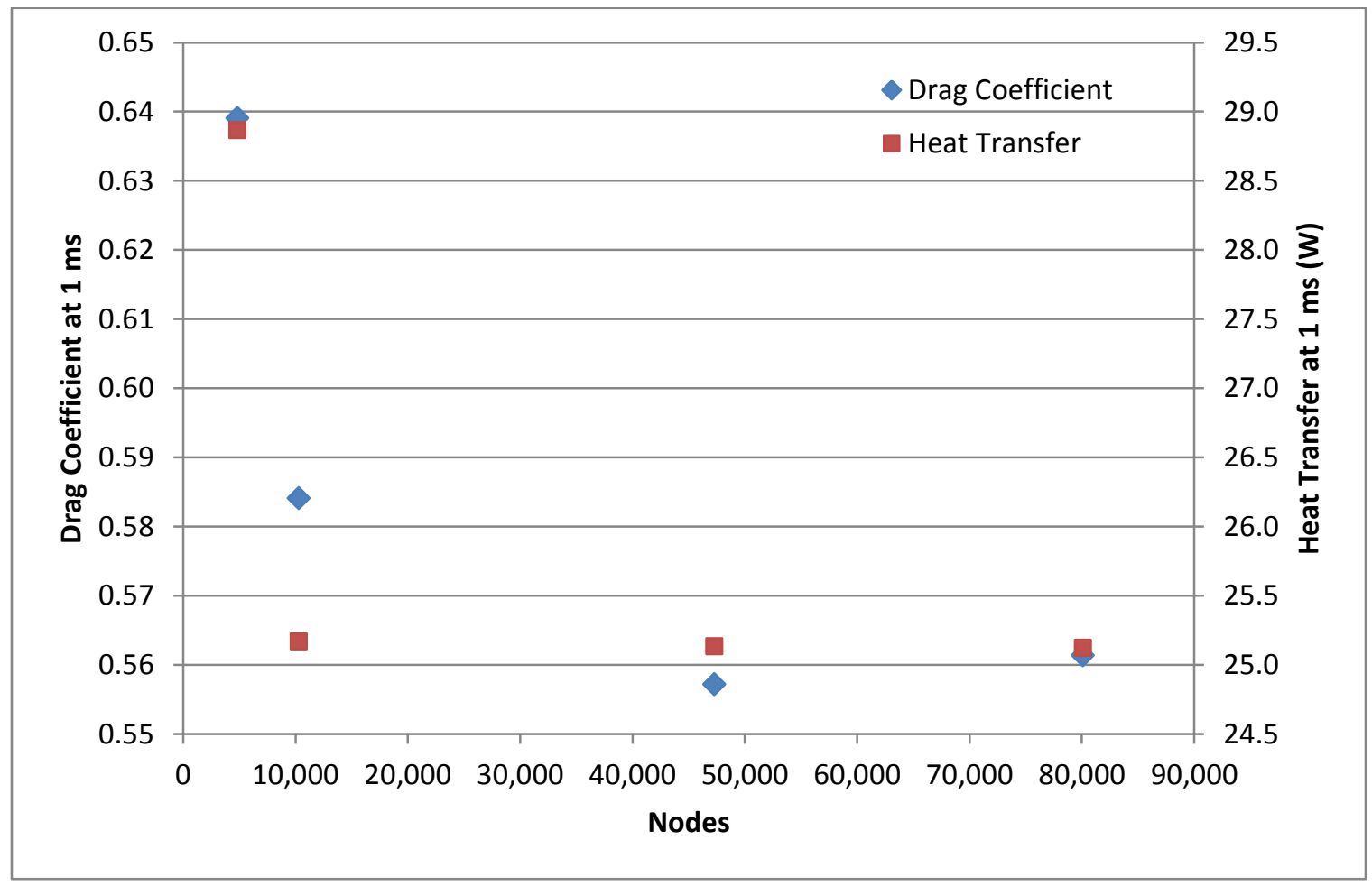

Figure 9. 2D, Axisymmetric Swirl Target Mesh Convergence Plot 


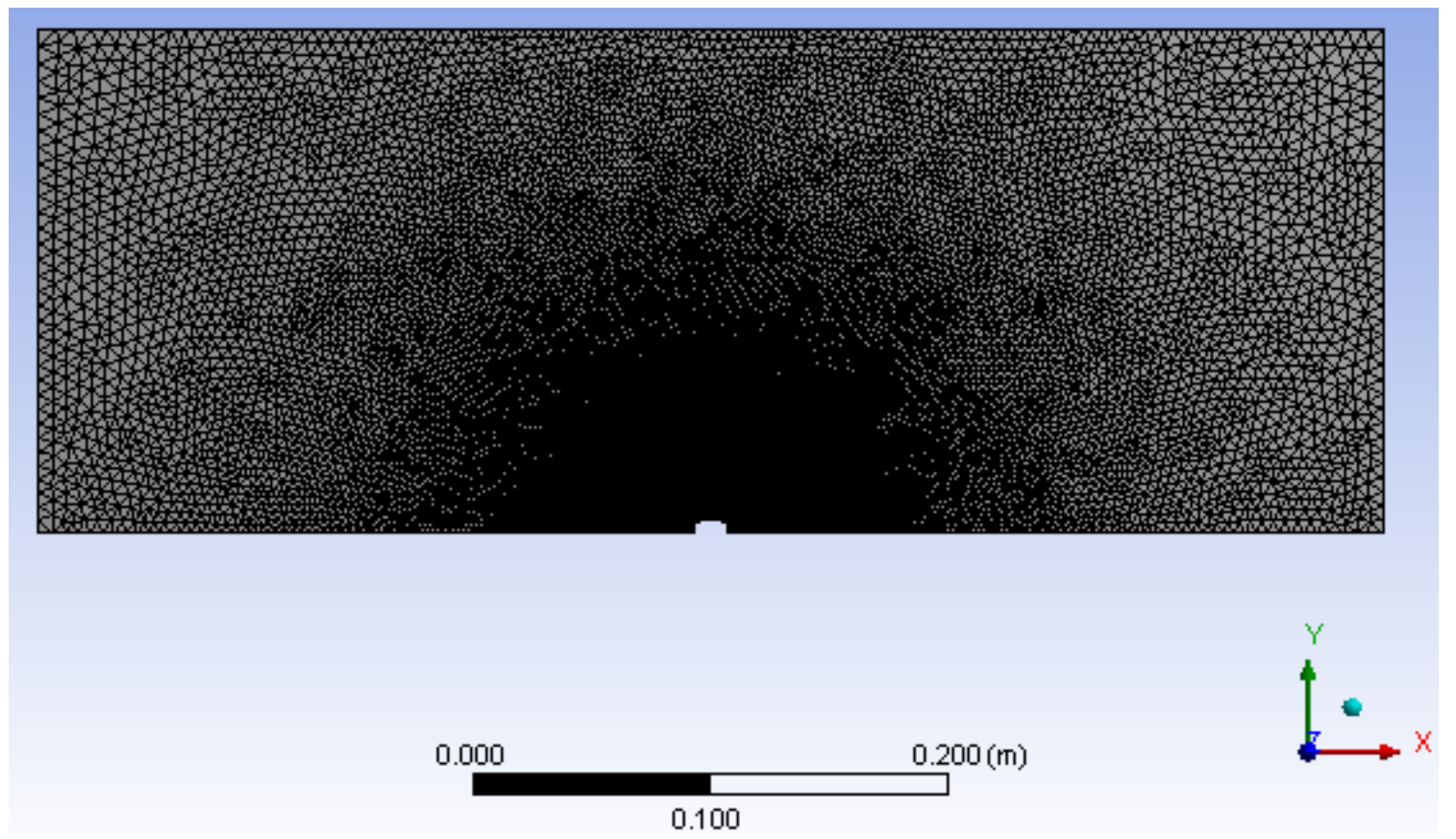

Figure 10. Entire View of 2D, Axisymmetric Target Mesh

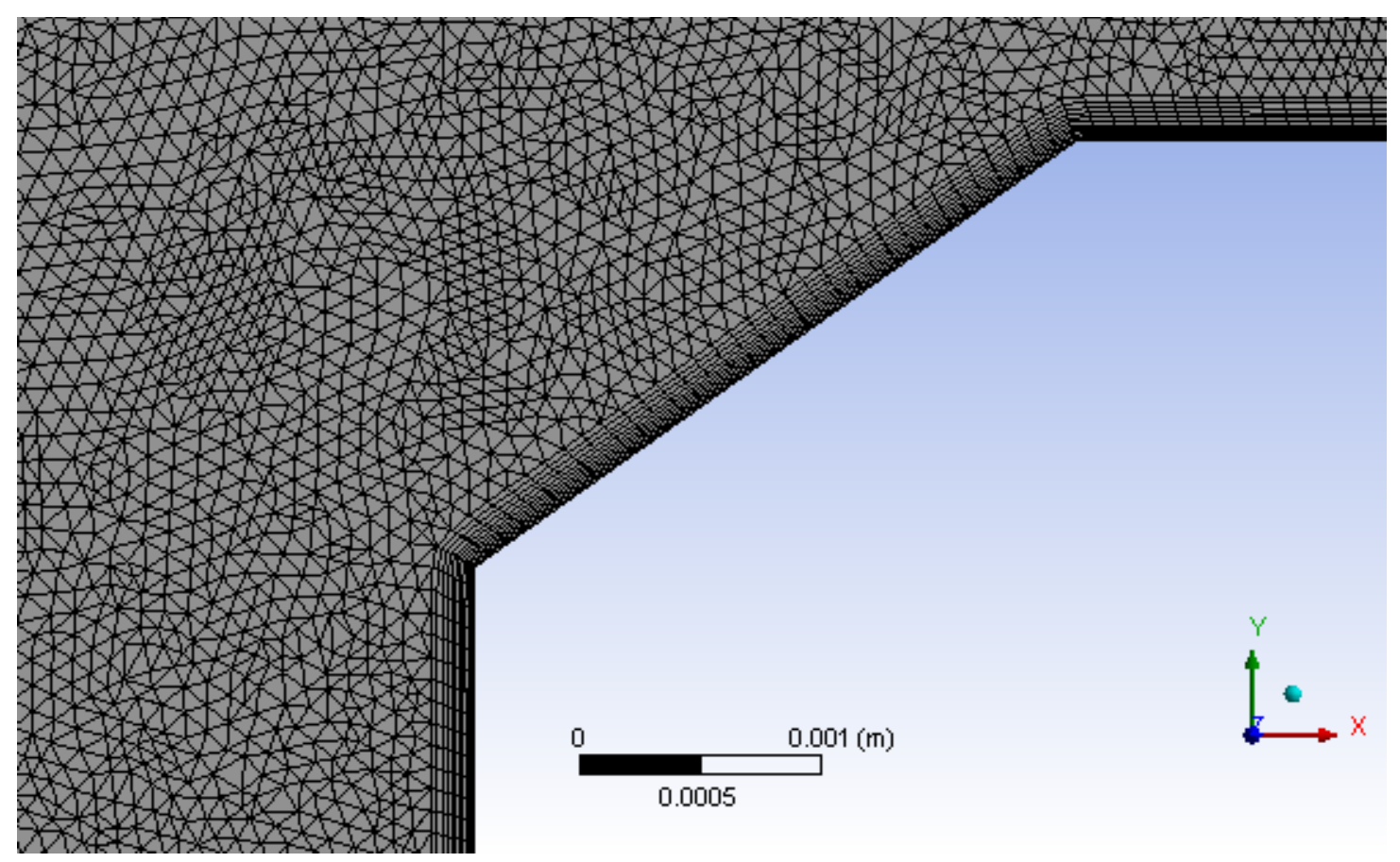

Figure 11. Inflation Layer View of 2D, Axisymmetric Target Mesh 
Finally, the initial guess for the time step size, $5.0 \mathrm{E}-7 \mathrm{~s}$, was evaluated. The drag coefficient and heat transfer results from a case with the same domain and mesh but with a smaller time step $(2.0 \mathrm{E}-7 \mathrm{~s})$ were compared to those from the initial case. Table 11 shows the minimal effect of time step size on both drag and heat transfer at $1 \mathrm{~ms}$. Reducing the original time step by more than half changed the drag coefficient and heat transfer at $1 \mathrm{~ms}$ by less than $0.7 \%$. Therefore, the original time step, $5.0 \mathrm{E}-7 \mathrm{~s}$, is adequate for capturing transient effects of the flow around a target.

Table 11. Time Step Size Study on 2D, Axisymmetric Swirl, Transient Drift Flow over Target

\begin{tabular}{|c|c|c|c|c|c|c|}
\hline Case & $\begin{array}{c}\text { Time } \\
\text { Step (s) }\end{array}$ & $\begin{array}{c}\text { Drag } \\
\text { Force (N) } \\
\text { at 1 ms }\end{array}$ & $\begin{array}{c}\text { Drag } \\
\text { Coefficient } \\
\text { at 1 ms }\end{array}$ & $\begin{array}{c}\text { Drag \% } \\
\text { Difference } \\
\text { with Case 1 }\end{array}$ & $\begin{array}{c}\text { Heat } \\
\text { Transfer } \\
\text { (W) at 1 ms }\end{array}$ & $\begin{array}{c}\text { Heat Transfer } \\
\text { \% Difference } \\
\text { with Case 1 }\end{array}$ \\
\hline 1 & $5.00 \mathrm{E}-07$ & 0.1512 & 0.5572 & NA & 24.97 & NA \\
\hline 2 & $2.00 \mathrm{E}-07$ & 0.1522 & 0.5610 & 0.672 & 25.13 & 0.673 \\
\hline
\end{tabular}

The domain size, mesh refinement, and time step studies have shown the initial case is capable of providing relatively precise drag and heat transfer values after simulating only $1 \mathrm{~ms}$. The results presented in the 2D, axisymmetric swirl section are the results from this initial transient, 2D, axisymmetric swirl target case. 


\section{D Drift Regime: Fluent Setup}

It was necessary to develop a 3D model of the flow around the target in the drift region. Comparison of the results from the $3 \mathrm{D}$ model and the $2 \mathrm{D}$, axisymmetric swirl model discussed in the previous sections would determine if the flow over the target in the drift region can adequately be modeled as axisymmetric. Additionally, analyzing the target's flight stability at different angles of attack could only be accomplished with a 3D model. Due to the large number of nodes, the 3D simulations were run across 120 parallel processors on LLNL clusters. All simulations presented in the 3D drift regime sections had residual drops greater than 4 orders of magnitude unless otherwise specified.

Much of the setup discussed in the Fluent setup section for 2D, axisymmetric swirl model applies to the 3D case setup as well. Since the 2D, axisymmetric swirl model indicated the flow reaches a steady state after roughly $0.8 \mathrm{~ms}$ and the flight through the drift region is approximately $16 \mathrm{~ms}$ (see 2D, axisymmetric drift results section), the 3D cases were set up as steady-state. As with the 2D, axisymmetric swirl case, a laminar case was also attempted in $3 \mathrm{D}$, but the continuity residual grew to unusable values. Therefore, a k-omega SST turbulence model was also implemented. Table 24 in Appendix A summarizes the Fluent case inputs for the 3D target model in the drift region.

Due to limited access to high performance computing licenses, only $13 \mathrm{D}$ simulation could run at any given time. Keeping the deadline in mind, it was recommended to base the mesh refinement and domain size on results from the $2 \mathrm{D}$, axisymmetric swirl studies in the previous section. Therefore, the domain size from the 2D, axisymmetric swirl model was adopted: 20 body lengths before, 15 body lengths above, and 20 body lengths behind the target. The fluid region was created by sketching 
the $2 \mathrm{D}$, axisymmetric domain in the $\mathrm{x}-\mathrm{y}$ plane and revolving it $360^{\circ}$ about the $\mathrm{x}$-axis. The refinement of this domain was limited by the $8 \mathrm{~GB}$ of memory available on the computer used for meshing. As with the 2D, axisymmetric mesh, 12 inflation layers with a 1.10 growth rate and default transition ratio of 0.272 were used. However, the overall growth rate was limited to 1.03 and the target face size was limited to $1 \mathrm{E}-4 \mathrm{~m}$ by the memory restrictions of the computer. Note that a tetrahedral-dominated meshing method was selected along with advanced size function on proximity and curvature. This mesh provided 5,450,161 nodes, and it is pictured in Figures 12-13.

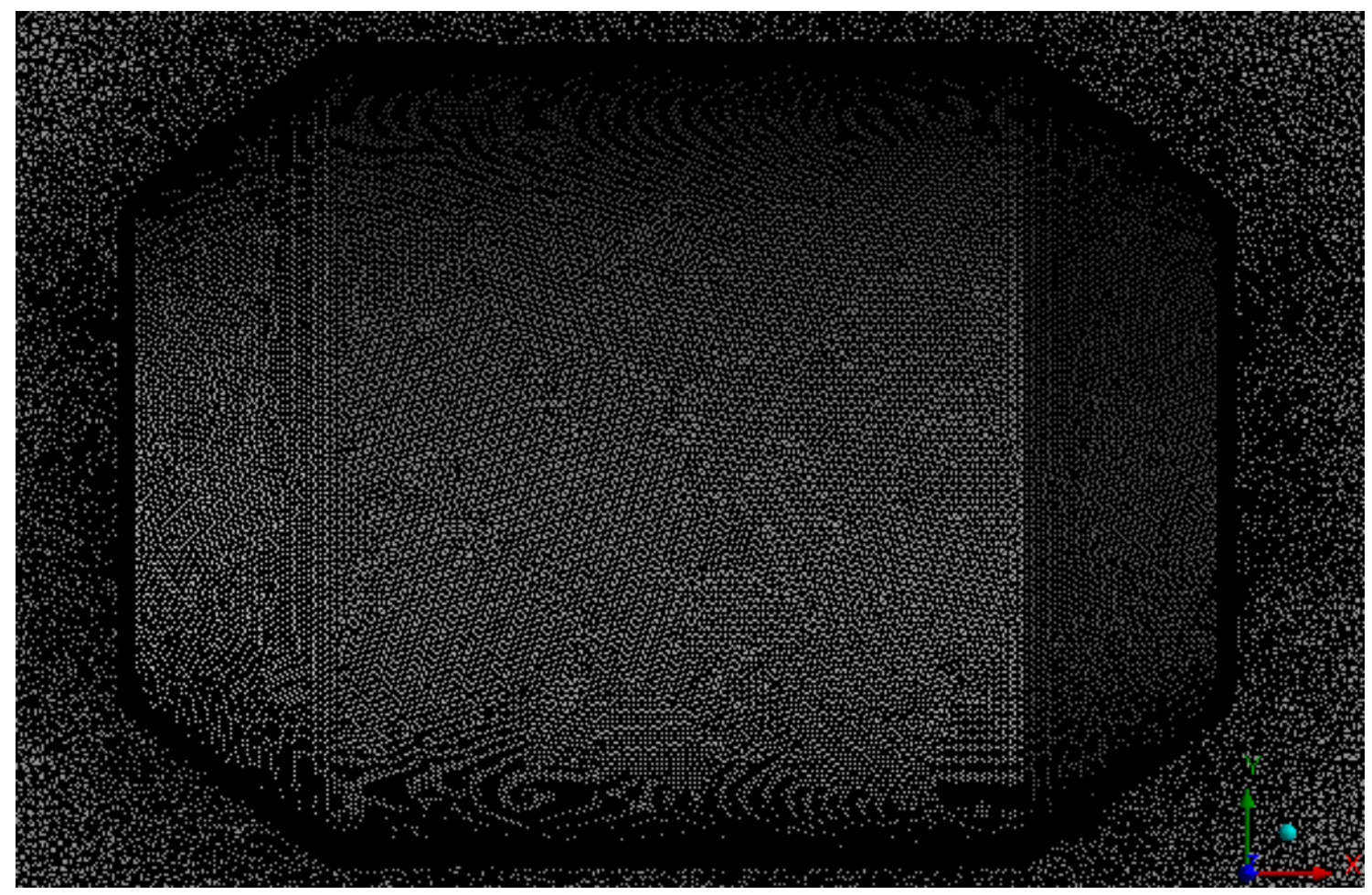

Figure 12. Zoomed-in View of Target in 3D Target Mesh Sliced at X-Y Plane 


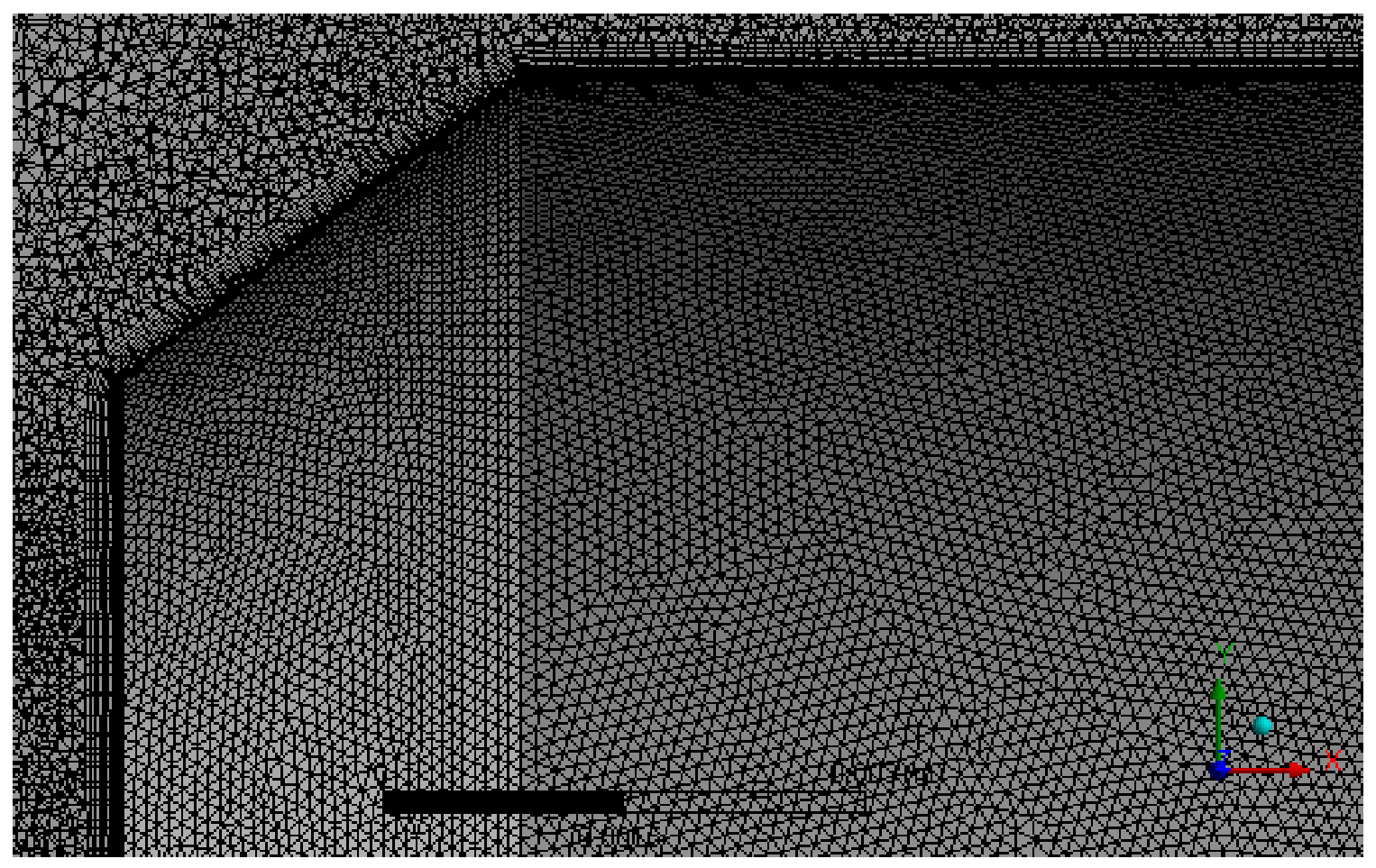

Figure 13. Inflation Layer View of 3D Target Mesh Sliced at X-Y Plane

To determine the Nusselt number and drag coefficient functions of the Reynolds number, simulations at various Reynolds numbers were run. To achieve the different Reynolds numbers, the velocity was varied between cases. The velocity was used to calculate the inlet Mach number, which was an input for the far-field pressure inlet boundary condition. The total temperature was also calculated for each case using equation 26 and used as the backflow total temperature in the pressure outlet boundary condition. See Table 12 for the velocity, Mach number, and total temperature for each case corresponding to a different Reynolds number. 
Table 12. Velocities and Mach Numbers for 3D, Steady-State Target Simulations with Different Reynolds Numbers

\begin{tabular}{|c|c|c|c|c|}
\hline Case & Reynolds Number & Velocity $(\mathbf{m} / \mathbf{s})$ & Mach Number & Total Temperature (K) \\
\hline 1 & 3937 & 250.0 & 0.75097 & 1247.4 \\
\hline 2 & 2400 & 152.74 & 0.45883 & 1123.7 \\
\hline 3 & 2800 & 178.2 & 0.53530 & 1150.3 \\
\hline 4 & 3200 & 212.6 & 0.63863 & 1192.7 \\
\hline 5 & 3600 & 229.12 & 0.688244 & 1215.8 \\
\hline 6 & 4400 & 280.03 & 0.841187 & 1297.7 \\
\hline 7 & 4800 & 305.49 & 0.917659 & 1344.7 \\
\hline 8 & 5200 & 330.95 & 0.99413 & 1395.9 \\
\hline 9 & 5600 & 356.4 & 1.0706 & 1451.2 \\
\hline
\end{tabular}

The flight stability of the target was analyzed by examining the forces and moments on the target at different angles of attack. The target was rotated $1^{\circ}, 2^{\circ}, 5^{\circ}, 10^{\circ}$, $15^{\circ}$, and $20^{\circ}$ from the axis parallel to the free-stream flow. To create these simulations, an axisymmetric, rectangular sketch of the overall domain was created in the $x-y$ plane and revolved $360^{\circ}$ about the $\mathrm{x}$-axis. Then, an axisymmetric sketch of the angled target was drawn in the $x-y$ plane. This target sketch was rotated $360^{\circ}$ about its own axis of symmetry and cut from the original domain. Due to the target rotation, a new centerline for the spin axis needed to be defined for each angle of attack case. These axes can be seen in Table 13. Each of these cases had the same meshing parameters as the original $0^{\circ}$ angle of attack case with 5,450,161 nodes. A second set of simulations were run at these same angles without spin to show the effect of spin on aerodynamic forces and moments.

Table 13. 3D, Steady-State Target Spin Axis for Varying Angle of Attack Cases

\begin{tabular}{|c|c|c|}
\hline Case & Angle of Attack (degrees) & $\begin{array}{c}\text { End Point of the Spin Axis } \\
\text { Defined from }(\mathbf{0 , 0 , 0 )}\end{array}$ \\
\hline 1 & 1 & $(0.00689895,-0.000120422,0)$ \\
\hline 2 & 2 & $(0.0068958,-0.00024081,0)$ \\
\hline 3 & 5 & $(0.0068737,-0.000601375,0)$ \\
\hline 4 & 10 & $(0.0067952,-0.0011982,0)$ \\
\hline 5 & 15 & $(0.0066649,-0.0017859,0)$ \\
\hline 6 & 20 & $(0.0064839,-0.0023599,0)$ \\
\hline
\end{tabular}




\section{RESULTS}

Sphere Validation: Results

The results of the 2D, axisymmetric sphere simulations described in the CFD chapter are presented in this section. The detailed Fluent case setup for these simulations is provided in Table 22 in Appendix A. Since the Roos and Willmarth [24] experiment results were one of the sources of comparison for the sphere drag coefficient, the velocities used in the Fluent simulations were calculated to result in the same Reynolds numbers from their experiments in the 20-130 Reynolds range. Table 14 and Figure 14 show the Fluent drag coefficients, Roos and Willmarth [24] drag coefficients, and Clift et al. [4] correlation drag coefficients at these Reynolds numbers. The percent difference between the Fluent drag coefficients and the 2 other published results can also be seen in Table 14. The Fluent drag coefficients had less than $0.5 \%$ difference when compared to the Clift et al. [4] correlation. When compared to the Roos and Willmarth [24] experimental data, the Fluent simulations had maximum and minimum percent differences of $9.37 \%$ and $-6.68 \%$, respectively. However, the average percent difference was only $-0.21 \%$, indicating a random scattering. Recall that Roos and Willmarth predicted a maximum error of $5 \%$ in their drag calculations due to experimental fluctuations. It appears that their experimental data lacks a high level of precision. A graph displaying the spread of the percent difference with the 2 published sources can be seen in Figure 15. Based on the close alignment with the Clift et al. [4] correlation and low average percent difference with the Roos and Willmarth [24] experimental data, the Fluent simulations were very successful at predicting the drag on a sphere with drift flow conditions. 
Table 14. Sphere Drag Coefficient Comparison

\begin{tabular}{|c|c|c|c|c|c|}
\hline \multirow{2}{*}{ Re } & \multicolumn{3}{|c|}{ Drag Coefficient } & \multicolumn{2}{c|}{ \% Difference } \\
\cline { 2 - 6 } & Fluent & $\begin{array}{c}\text { Roos and Willmarth } \\
\text { Experiment }\end{array}$ & $\begin{array}{c}\text { Clift Correlation } \\
\mathbf{2 0 < \text { Re<260 }}\end{array}$ & $\begin{array}{c}\text { with } \\
\text { Experiment }\end{array}$ & $\begin{array}{c}\text { with Clift } \\
\text { Correlation }\end{array}$ \\
\hline 21.1 & 2.632 & 2.82 & 2.643 & -6.680 & -0.414 \\
\hline 23.4 & 2.467 & 2.48 & 2.474 & -0.518 & -0.289 \\
\hline 29.1 & 2.160 & 2.28 & 2.161 & -5.268 & -0.065 \\
\hline 45 & 1.675 & 1.79 & 1.671 & -6.441 & 0.219 \\
\hline 50.6 & 1.568 & 1.58 & 1.564 & -0.769 & 0.262 \\
\hline 54.4 & 1.506 & 1.52 & 1.502 & -0.916 & 0.281 \\
\hline 68.9 & 1.324 & 1.34 & 1.320 & -1.157 & 0.315 \\
\hline 78.2 & 1.238 & 1.27 & 1.234 & -2.489 & 0.316 \\
\hline 88.1 & 1.164 & 1.12 & 1.160 & 3.891 & 0.306 \\
\hline 93.8 & 1.126 & 1.03 & 1.123 & 9.367 & 0.296 \\
\hline 101 & 1.085 & 1.08 & 1.082 & 0.424 & 0.282 \\
\hline 104 & 1.069 & 1.05 & 1.066 & 1.764 & 0.275 \\
\hline 108 & 1.048 & 1.02 & 1.045 & 2.770 & 0.264 \\
\hline 109 & 1.043 & 1.03 & 1.041 & 1.299 & 0.262 \\
\hline 124 & 0.978 & 0.994 & 0.976 & -1.619 & 0.211 \\
\hline 130 & 0.955 & 0.927 & 0.953 & 3.036 & 0.186 \\
\hline
\end{tabular}

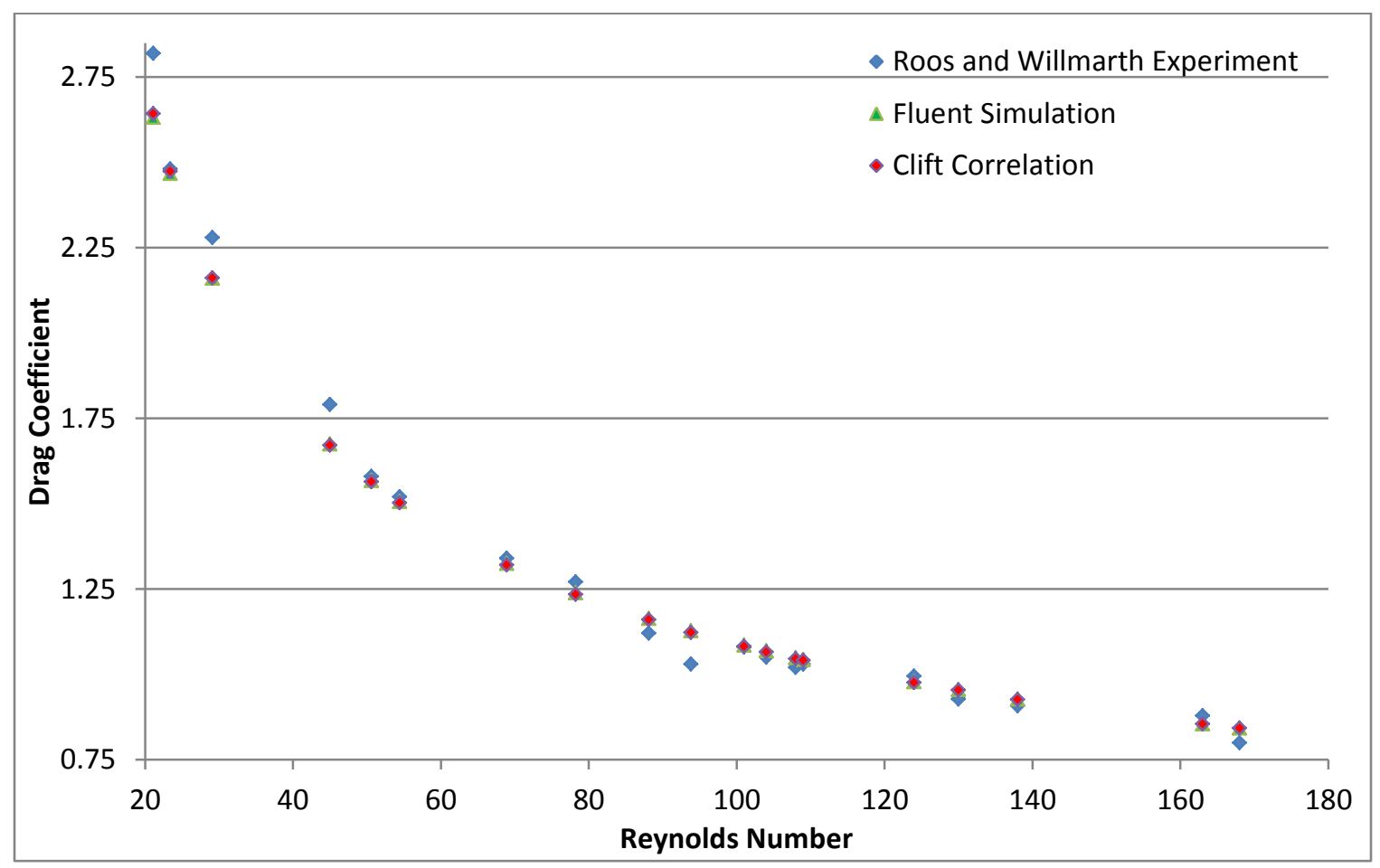

Figure 14. Sphere Drag Coefficient versus Reynolds Number for Experimental Data, a Published Correlation, and Fluent Simulation Results 


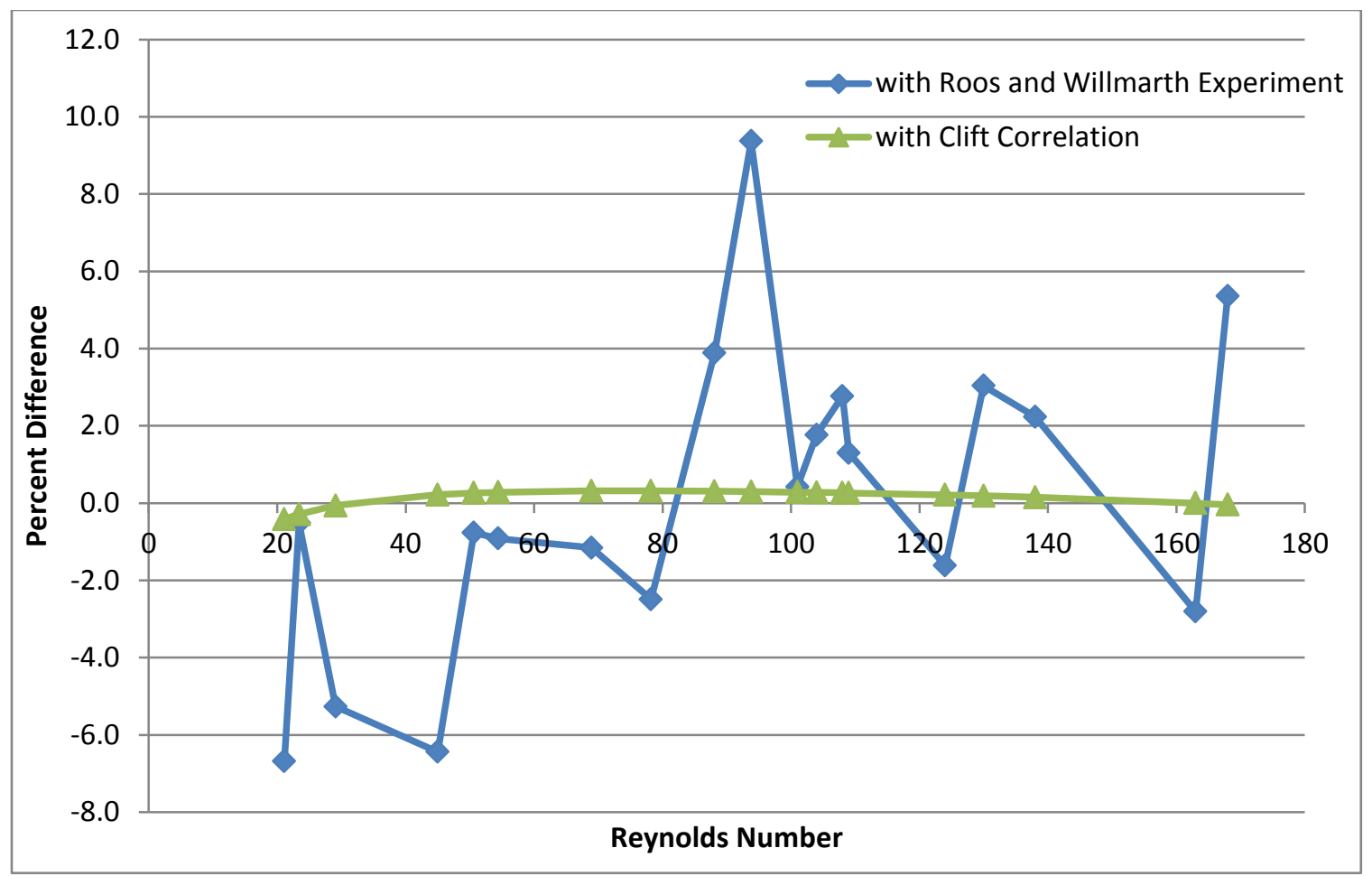

Figure 15. Graph of Percent Difference between Fluent Results and Experimental Data and a Published Correlation

The Fluent separation angle (measured from the front stagnation point to the separation point in an axisymmetric slice) was determined by identifying the separation point as the location of zero wall shear stress. To more closely approximate the location of separation, more nodes along the sphere wall were beneficial. Therefore, case 8 from the mesh refinement study (which had an edge size of $5 \mathrm{E}-5 \mathrm{~m}$, an overall growth rate of 1.02, and approximately 40,980 nodes) was used to produce the wall shear stress data. Figure 16 shows plots of the wall shear stress on the sphere from the front stagnation point to the back stagnation point for cases with different Reynolds numbers. The zero wall shear stress location for each case was estimated to be at the node closest to the smallest wall shear stress. (Note that the front and rear stagnation points were omitted from this minimum wall shear stress determination.) Since the nodes along the curved 
sphere wall were 5E-5 m apart, the separation angle had a maximum error of $\pm 0.29^{\circ}$ due to node spacing. Table 15 shows the comparison between the Fluent separation angles and the separation angles predicted by the Clift et al. [4] equation (see background section) at different Reynolds numbers. Notice that the Fluent separation angles are within $2.5 \%$ of the expected angles predicted by the correlation, and most are within 0.5\%. The largest discrepancies occurred at the lowest Reynolds numbers. Considering over half of the $0.5 \%$ difference can be attributed to node spacing alone, Fluent's ability to determine the separation location in this flow field is excellent.

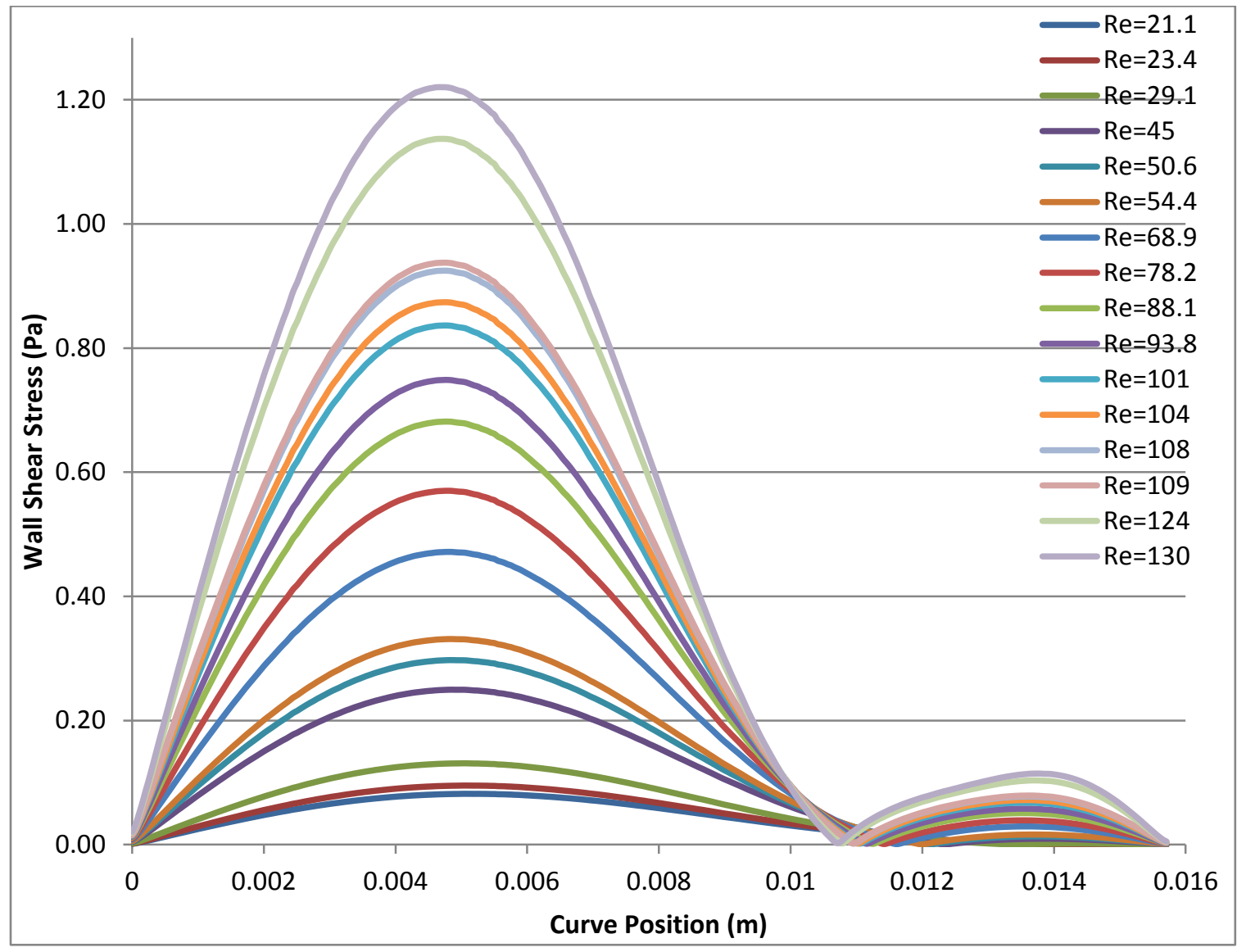

Figure 16. Wall Shear Stress Plot on Sphere at Different Reynolds Numbers 
Table 15. Sphere Separation Angles (from Front Stagnation Point to Separation Point) from Fluent Simulations and Clift Correlation at Different Reynolds Numbers

\begin{tabular}{|c|c|c|c|}
\hline \multirow{2}{*}{ Re } & \multicolumn{2}{|c|}{ Separation Angle (degrees) } & \multirow{2}{*}{$\begin{array}{c}\text { Difference with } \\
\text { Correlation }\end{array}$} \\
\cline { 2 - 3 } & Fluent & Clift Correlation & 2.376 \\
\hline 21.1 & 173.70 & 169.66 & 0.818 \\
\hline 23.4 & 163.95 & 162.62 & 0.064 \\
\hline 29.1 & 153.63 & 153.54 & 0.003 \\
\hline 45 & 141.60 & 141.59 & -0.198 \\
\hline 50.6 & 138.73 & 139.00 & 0.068 \\
\hline 54.4 & 137.58 & 137.49 & 0.068 \\
\hline 68.9 & 132.99 & 132.90 & 0.491 \\
\hline 78.2 & 131.27 & 130.63 & 0.300 \\
\hline 88.1 & 128.98 & 128.59 & 0.216 \\
\hline 93.8 & 127.83 & 127.56 & 0.259 \\
\hline 101 & 126.68 & 126.36 & 0.175 \\
\hline 104 & 126.11 & 125.89 & 0.193 \\
\hline 108 & 125.54 & 125.30 & 0.308 \\
\hline 109 & 125.54 & 125.15 & 0.056 \\
\hline 124 & 123.25 & 123.18 & 0.165 \\
\hline 130 & 122.67 & 122.47 & \\
\hline
\end{tabular}

The flow around the sphere was also analyzed by viewing the Fluent velocity contours and streamlines. Figures 17-32 show streamlines overlaid on velocity contour plots for the different Reynolds number cases. Notice that the velocity of the fluid increases as it passes over the front of the sphere. This occurs because the sphere does not have a negligible thickness (like a flat plate) [20], and the incompressible flow is forced through a smaller cross-sectional area when traveling around the front of the sphere. By continuity, this would require an increased fluid velocity [20]. By Bernoulli's equation, an increase in velocity of an incompressible fluid would result in a decrease in pressure [20]. Note that the opposite trend occurs on the back half of the sphere. The flow's cross-sectional area increases, which lowers the velocity and increases the pressure. This adverse pressure gradient coupled with the fluid's viscosity 
causes flow separation [20]. Also note that the wake is concave for the first 3 figures (where the Reynolds number is below 35) and convex for the remaining 13 figures (where the Reynolds number is above 35). This agrees well with Nakamura's [21] observations that the wake structure changes from concave to convex as the Reynolds number increases and that the transition occurs near a Reynolds number of 35 . Note that the separation is not apparent in the first 2 cases $(\operatorname{Re}=21.1$ and $\mathrm{Re}=23.4)$ in Figures 17 and 18. This is due to the low speed near the rear stagnation point and coarse selection of streamline points. By adding more streamlines and zooming in on the region behind the sphere, small wake regions are visible at both of these Reynolds numbers (see Figures 33 and 34). It is clear that out of these 16 cases, the case with the lowest Reynolds number $(\operatorname{Re}=21.1)$ has the smallest separation region. In the separation angle study, this case had the largest separation angle $\left(173.7^{\circ}\right)$, indicating a separation point only $6.3^{\circ}$ from the rear stagnation point. This aligns well with Clift's [4] estimation that separation appears near a Reynolds number of 20 .

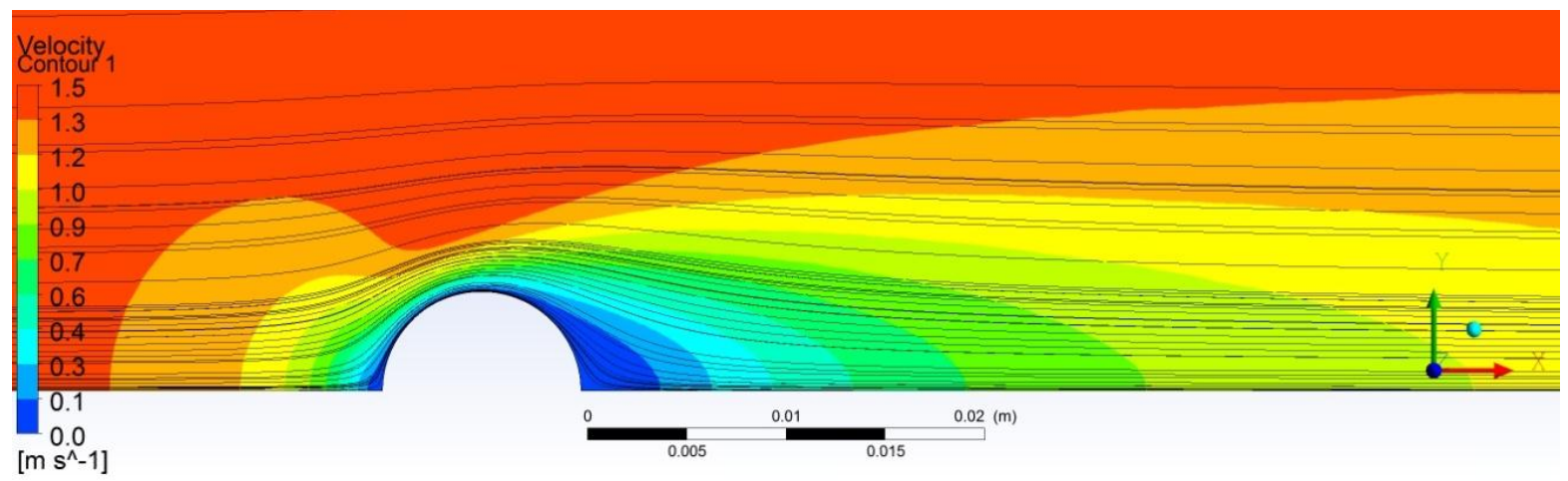

Figure 17. Sphere Velocity Contour with Streamlines Overlaid at $\mathbf{R e}=\mathbf{2 1 . 1}$ 


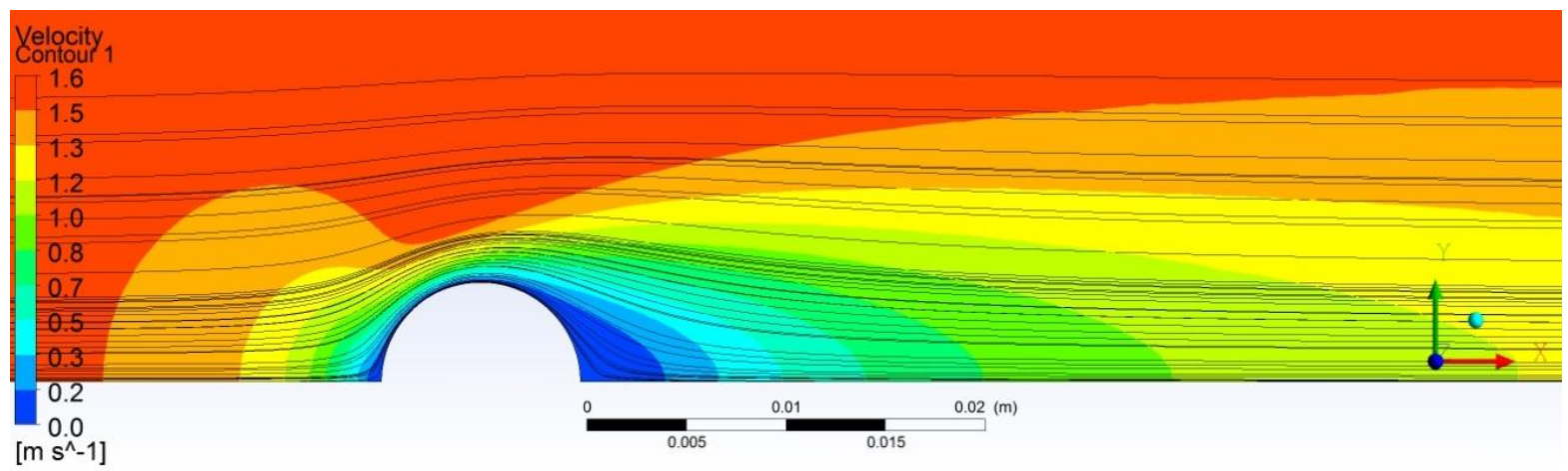

Figure 18. Sphere Velocity Contour with Streamlines Overlaid at $\mathbf{R e}=\mathbf{2 3 . 4}$

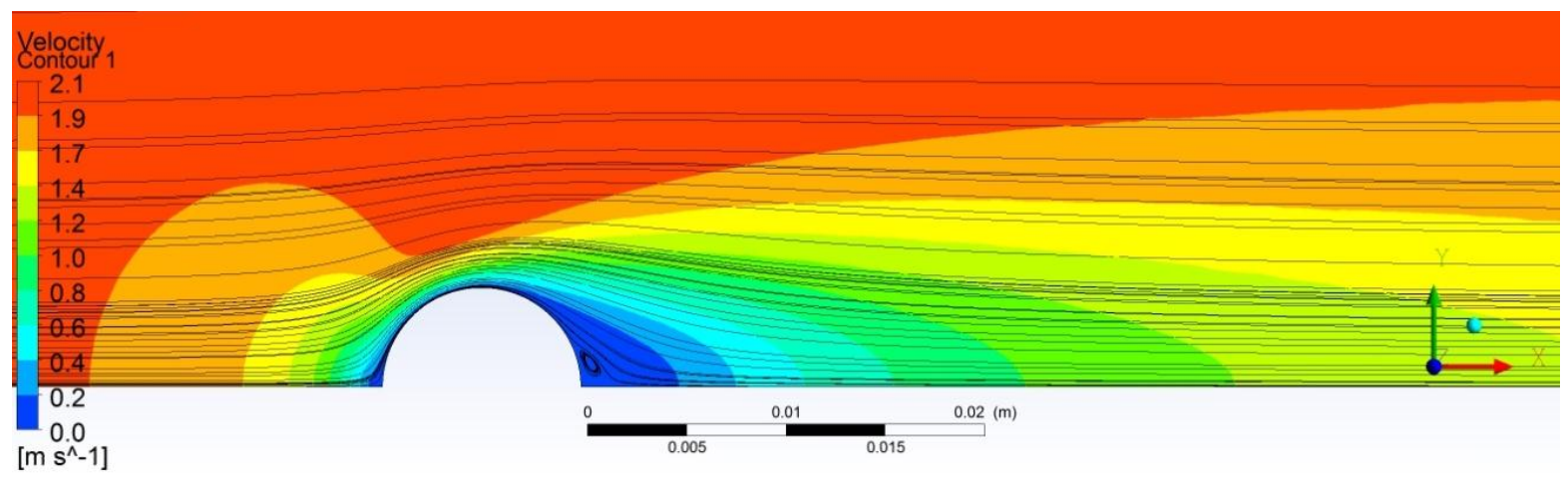

Figure 19. Sphere Velocity Contour with Streamlines Overlaid at $\operatorname{Re}=\mathbf{2 9} .1$

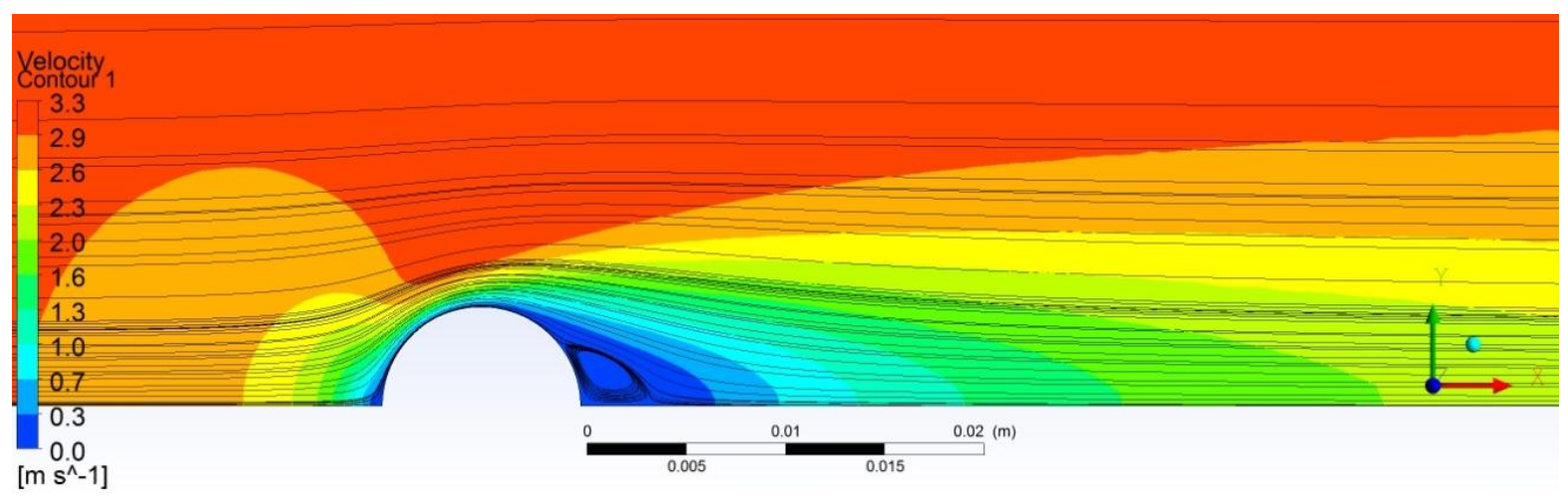

Figure 20. Sphere Velocity Contour with Streamlines Overlaid at $\mathrm{Re}=45$ 


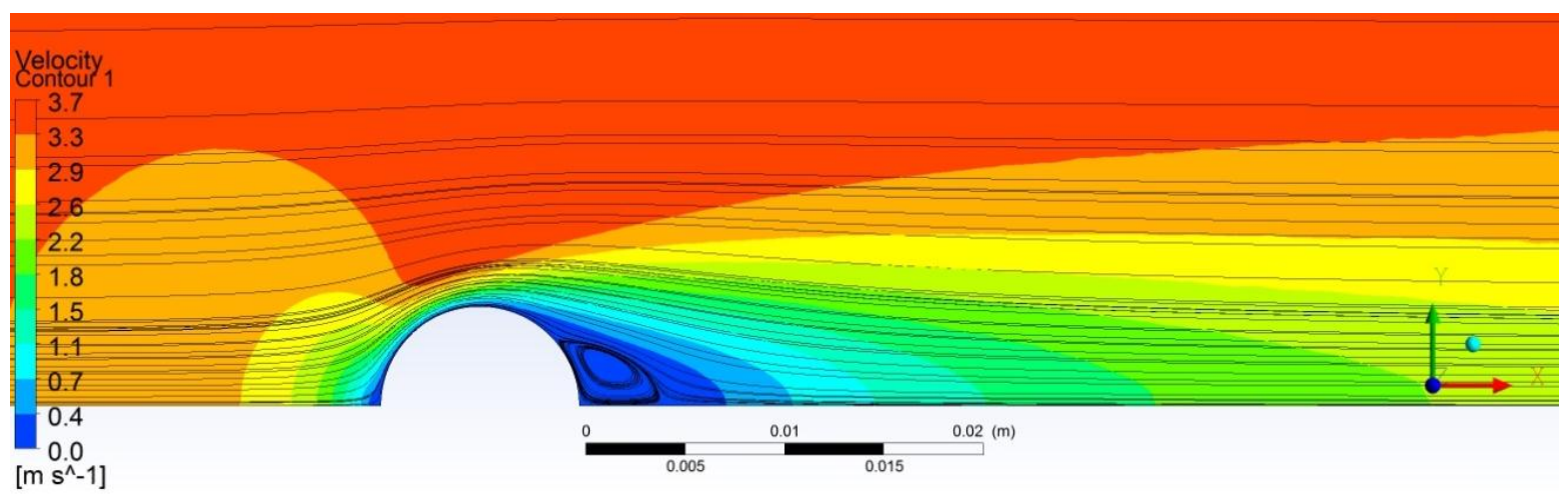

Figure 21. Sphere Velocity Contour with Streamlines Overlaid at $\mathbf{R e}=\mathbf{5 0 . 6}$

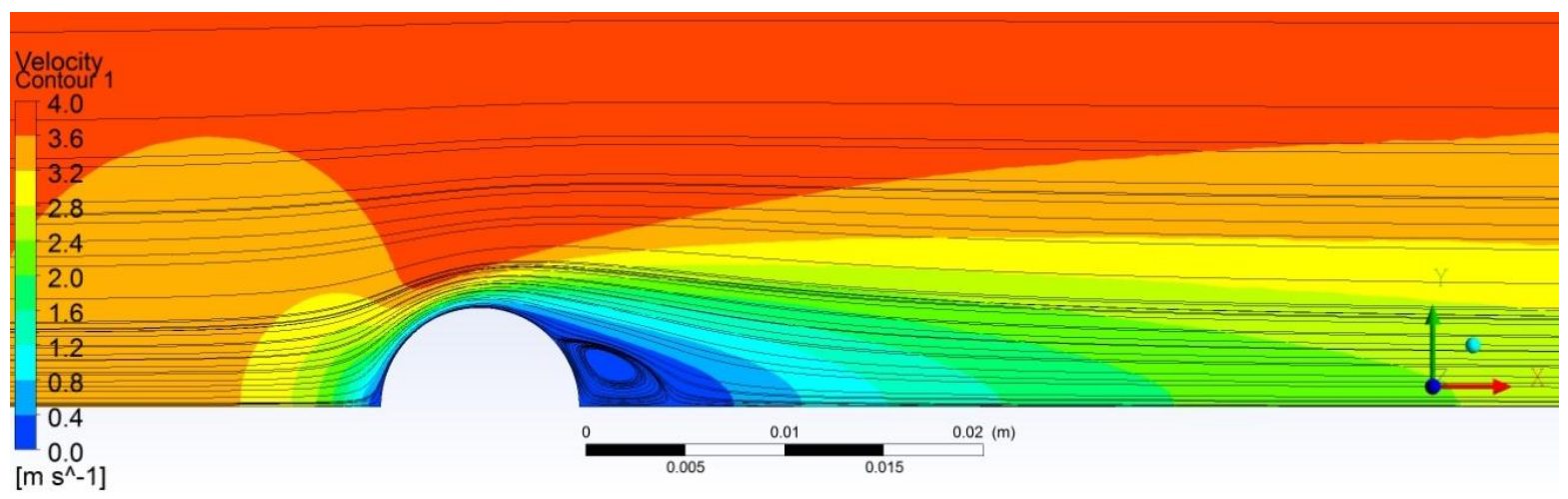

Figure 22. Sphere Velocity Contour with Streamlines Overlaid at $\mathbf{R e}=\mathbf{5 4 . 4}$

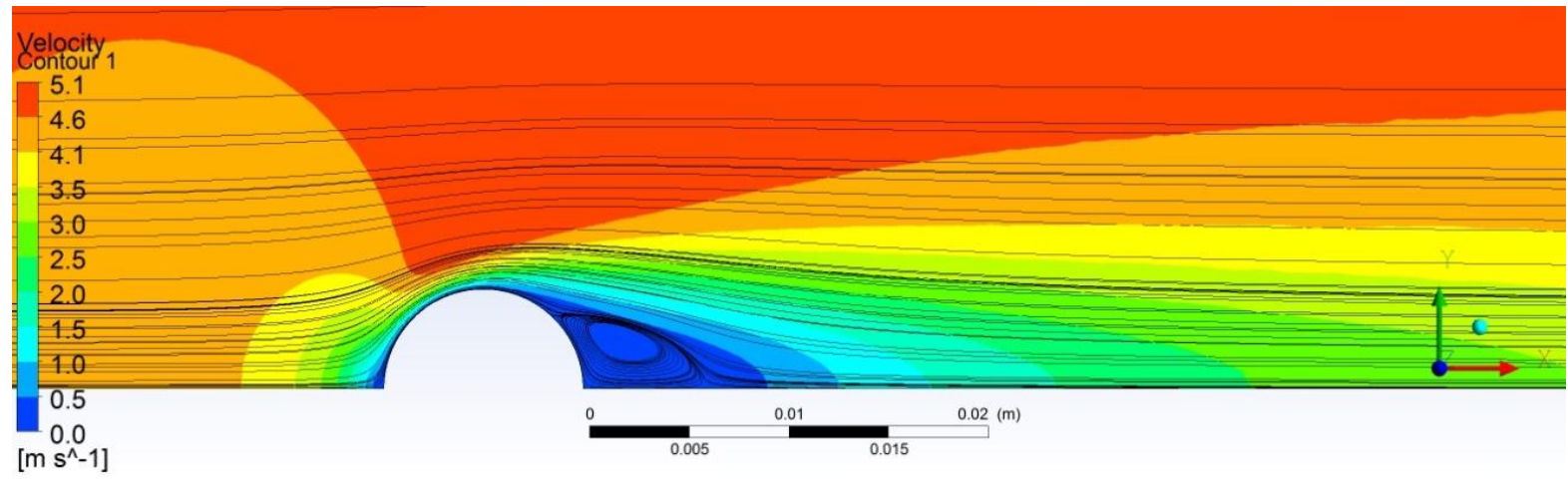

Figure 23. Sphere Velocity Contour with Streamlines Overlaid at $\mathrm{Re}=\mathbf{6 8 . 9}$ 


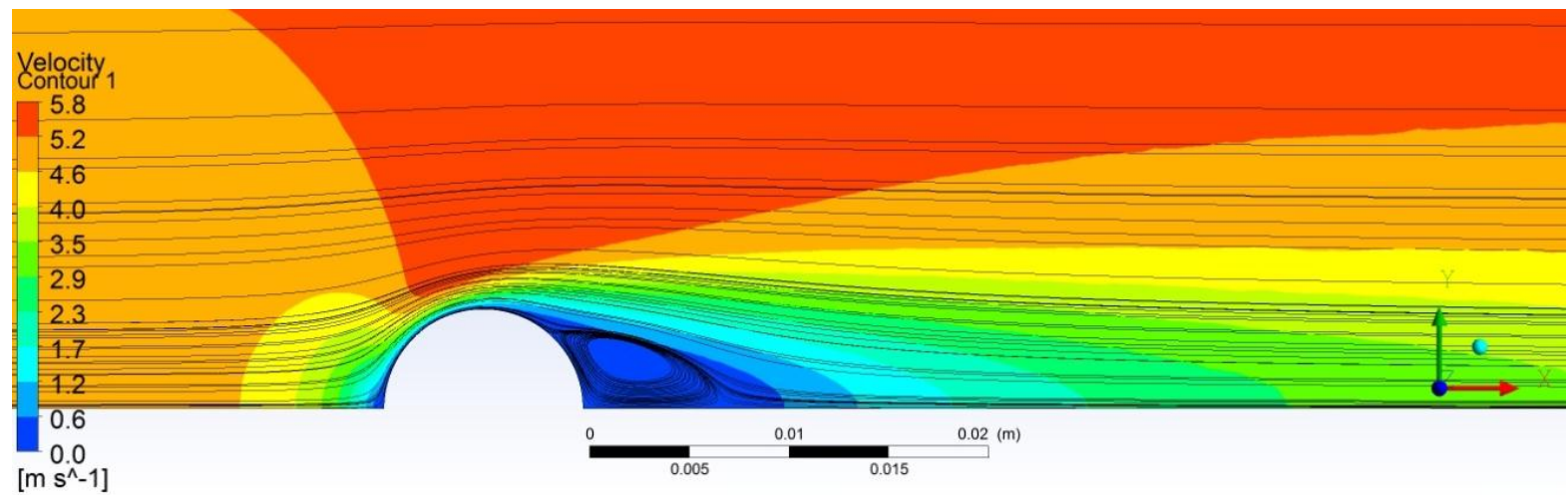

Figure 24. Sphere Velocity Contour with Streamlines Overlaid at $\operatorname{Re}=\mathbf{7 8 . 2}$

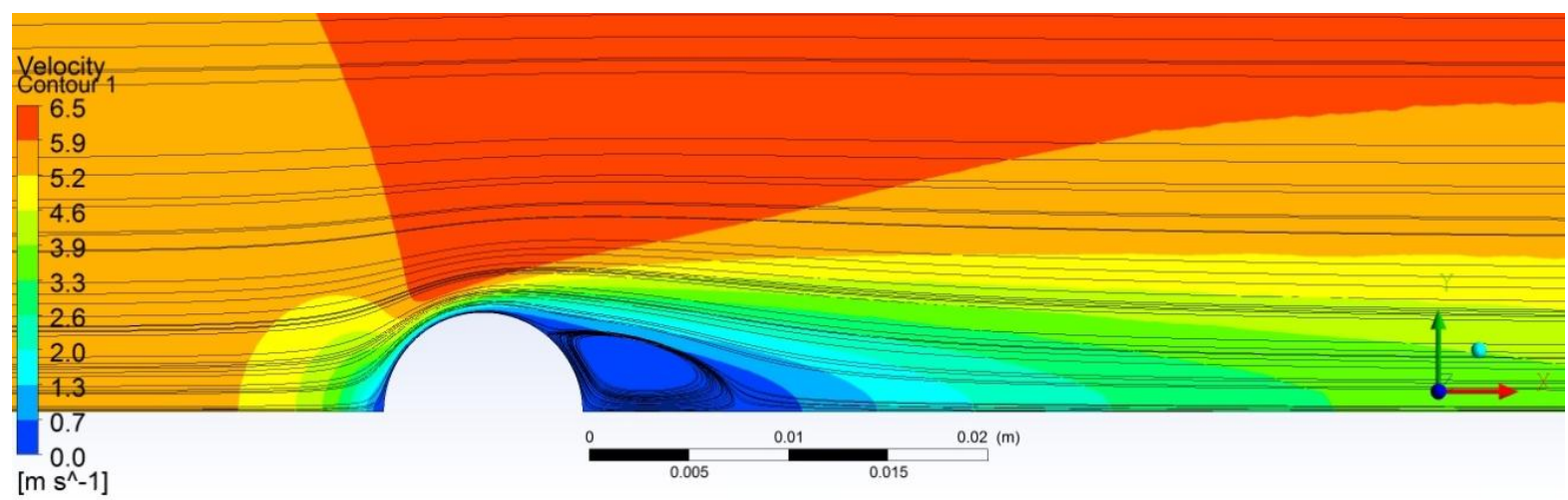

Figure 25. Sphere Velocity Contour with Streamlines Overlaid at $\mathbf{R e}=\mathbf{8 8 . 1}$

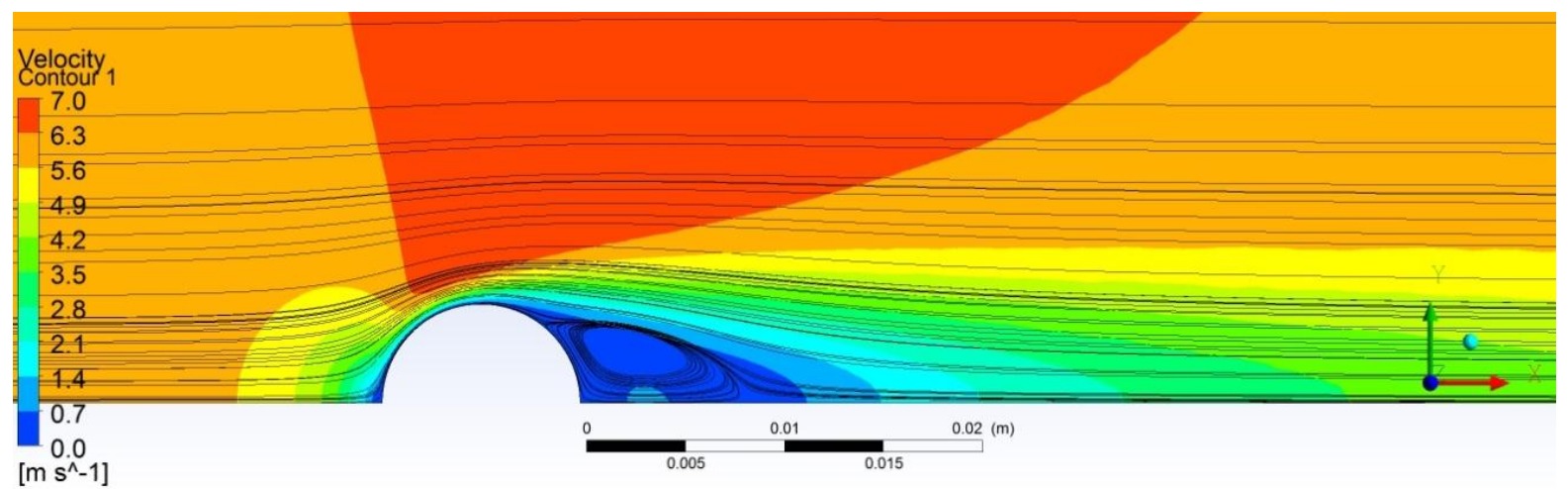

Figure 26. Sphere Velocity Contour with Streamlines Overlaid at $\operatorname{Re}=93.8$ 


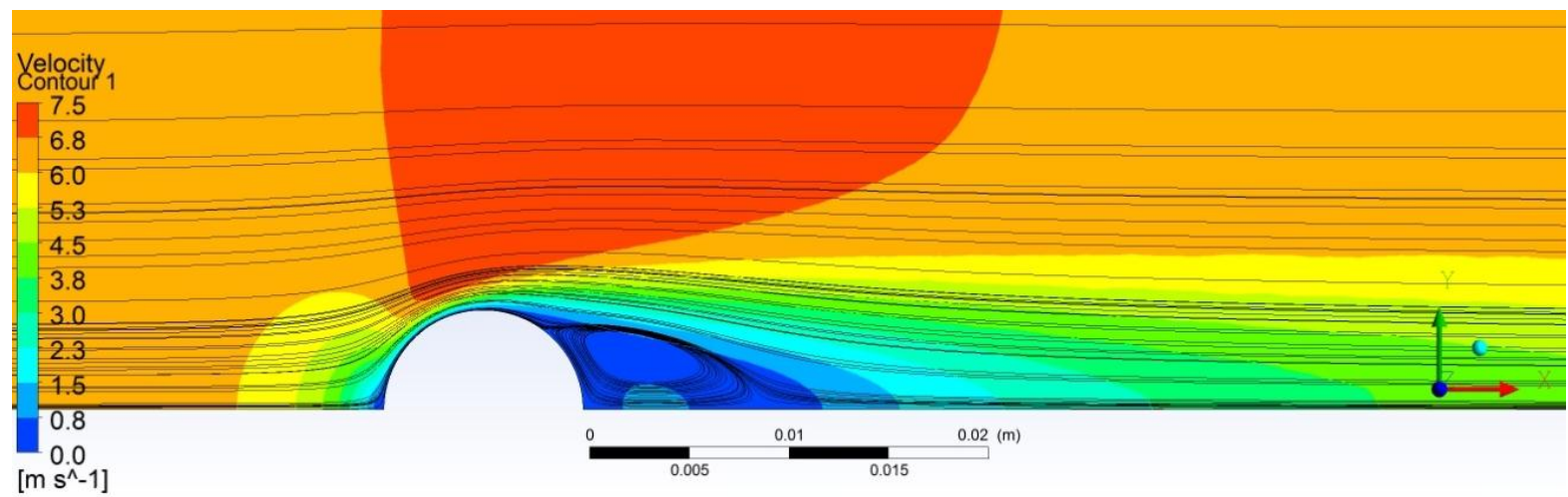

Figure 27. Sphere Velocity Contour with Streamlines Overlaid at $\mathbf{R e}=101$

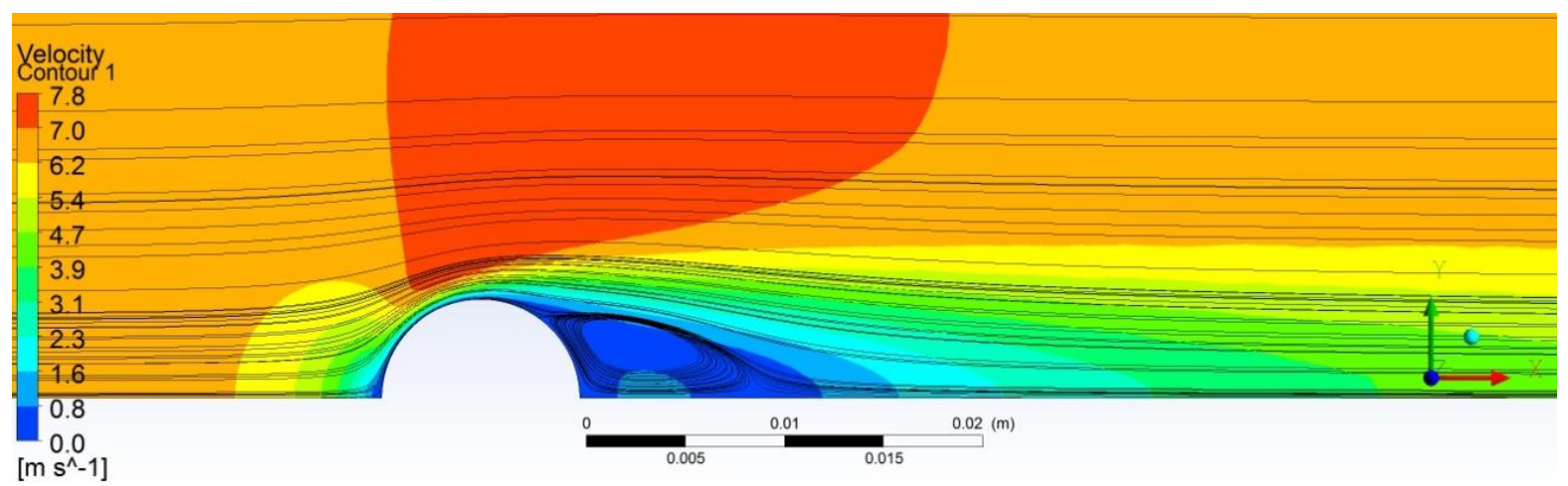

Figure 28. Sphere Velocity Contour with Streamlines Overlaid at $\mathrm{Re}=104$

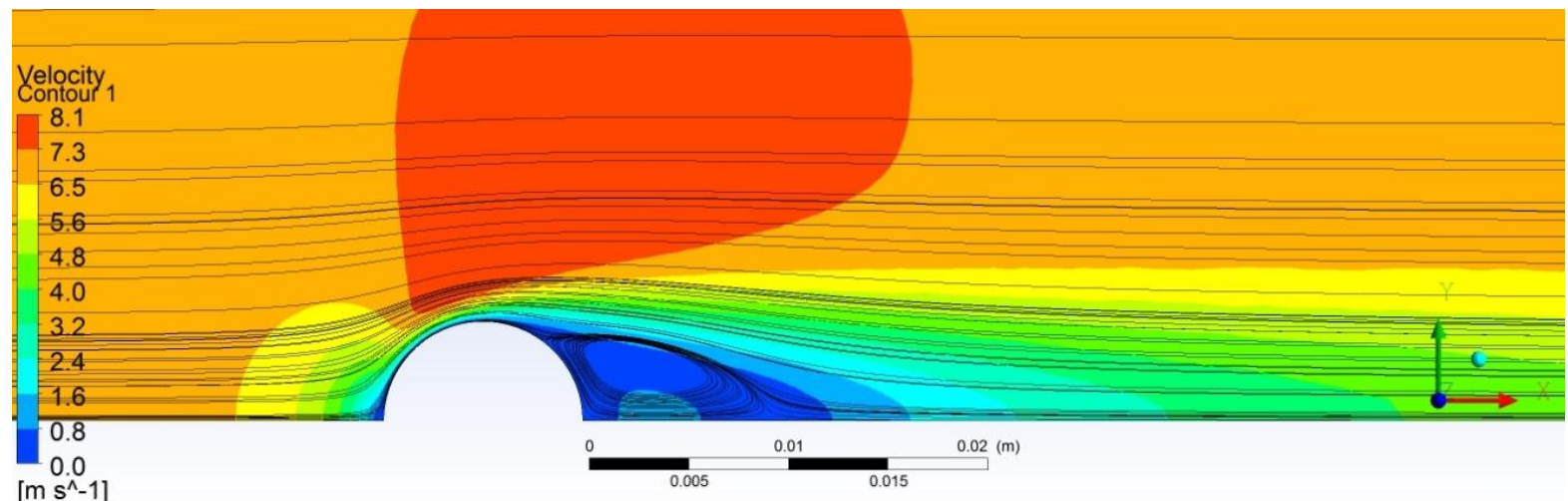

Figure 29. Sphere Velocity Contour with Streamlines Overlaid at $\mathbf{R e}=108$ 


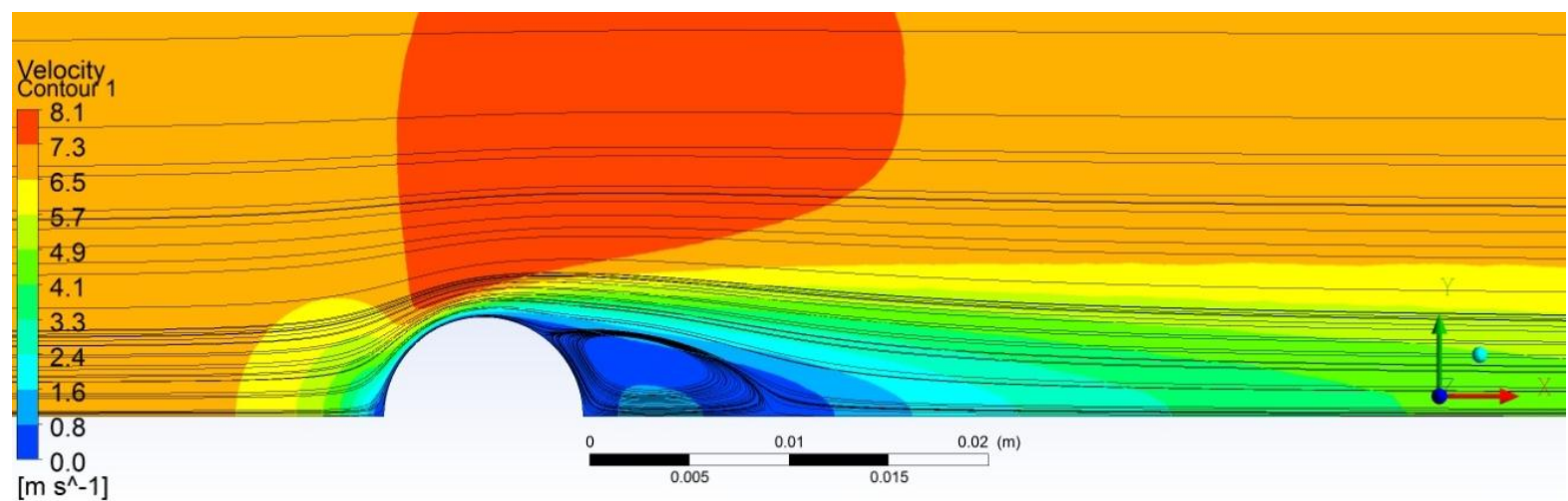

Figure 30. Sphere Velocity Contour with Streamlines Overlaid at $\mathbf{R e}=109$

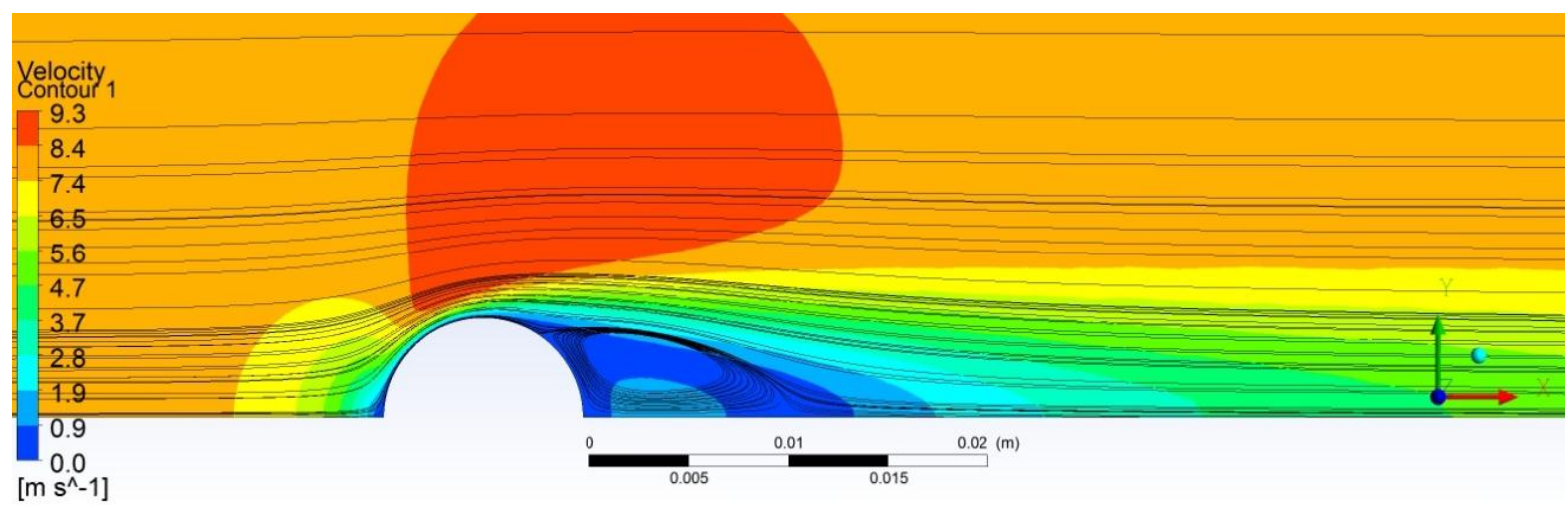

Figure 31. Sphere Velocity Contour with Streamlines Overlaid at $\mathbf{R e}=124$

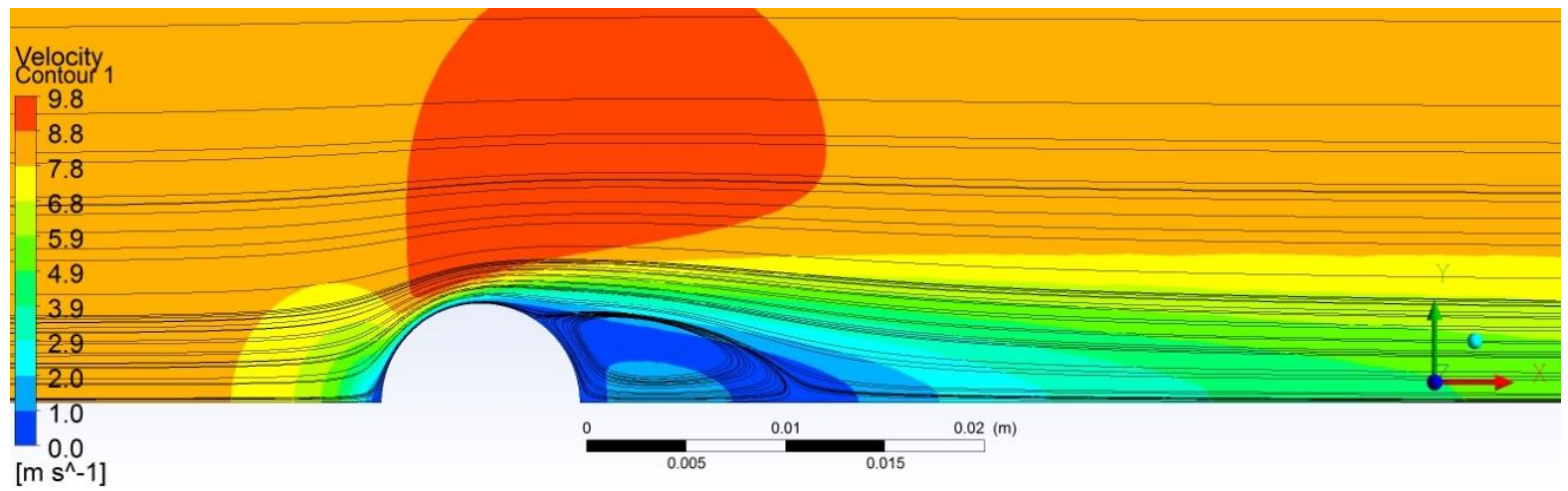

Figure 32. Sphere Velocity Contour with Streamlines Overlaid at $\mathbf{R e}=\mathbf{1 3 0}$ 


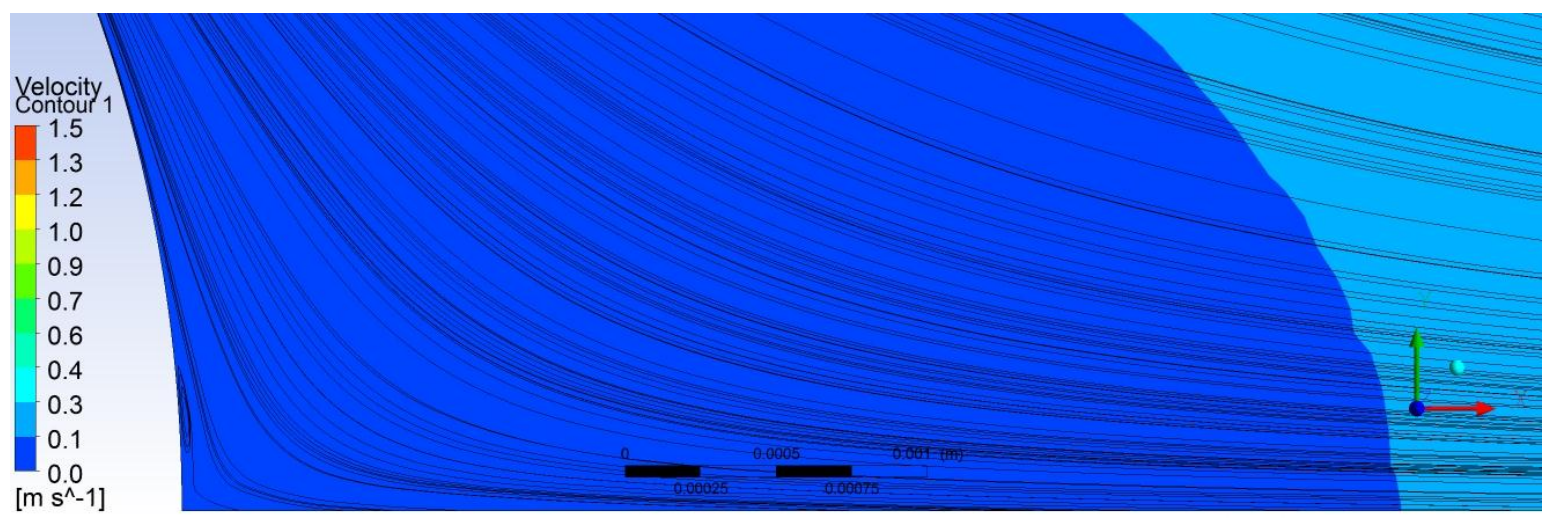

Figure 33. Zoomed-in View of Sphere Velocity Contour with Streamlines Overlaid at $\mathrm{Re}=\mathbf{2 1 . 1}$

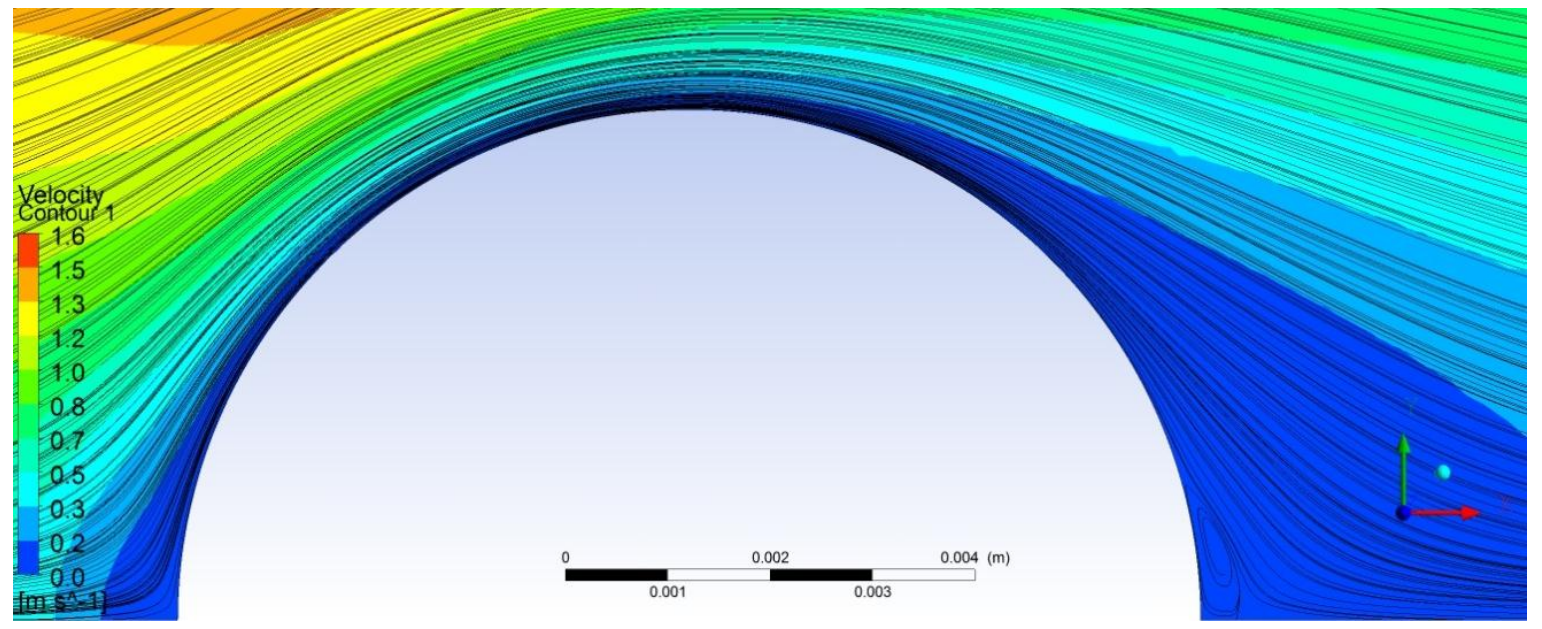

Figure 34. Zoomed-in View of Sphere Velocity Contour with Streamlines Overlaid at $\mathbf{R e}=\mathbf{2 3 . 4}$

Based on the previously discussed sphere results and their excellent alignment with published results for the drag coefficient, separation angle, and wake structure, the Fluent sphere simulations provided a high-level of accuracy. Fluent simulations of external flows over blunt objects with similar flow conditions, separation regions, fluid properties, assumptions, and mesh generation methods should also predict accurate results. 


\section{D, Axisymmetric Drift Regime: Results}

This section presents the results of the transient, 2D, axisymmetric swirl model of a target in the drift region as described in the $2 \mathrm{D}$, axisymmetric drift regime sections in the CFD chapter. A 47,285-node mesh consisting of 12 inflation layers with a 1.10 growth rate and a default transition ratio of 0.272 , an overall growth rate of 1.02 , a triangle-dominated meshing method, and a target edge size of 5E-5 m was created from an axisymmetric domain spanning 20 body lengths before, 15 body lengths above, and 20 body lengths behind. The Fluent case setup can be seen in Table 23 in Appendix A.

The velocity contours and streamlines were plotted at several times to visualize the flow and determine the existence of a steady-state solution. Figures 35-44 show the flow's development. Notice that the fluid velocity increases as it flows around the front of the target. Note that the simulations of incompressible flow around a sphere predicted this same velocity trend. As discussed in the sphere results section, the velocity increases because the flow is forced through a smaller cross-sectional area when traveling around the object. Additionally, a separation point occurs after the front of the obstacle just like in the flow over a sphere. Notice that the flow separates from the target near the beginning of the $3^{\text {rd }}$ target wall (the wall parallel with the free-stream flow). A small thin vortex appears after this separation point along the horizontal face. The wake behind the target appears to grow as time elapses. The wake appears to reach a steady state after approximately $0.8 \mathrm{~ms}$. The pressure and temperature contours at $1 \mathrm{~ms}$ can be seen in Figures 69 and 70 in Appendix B. 


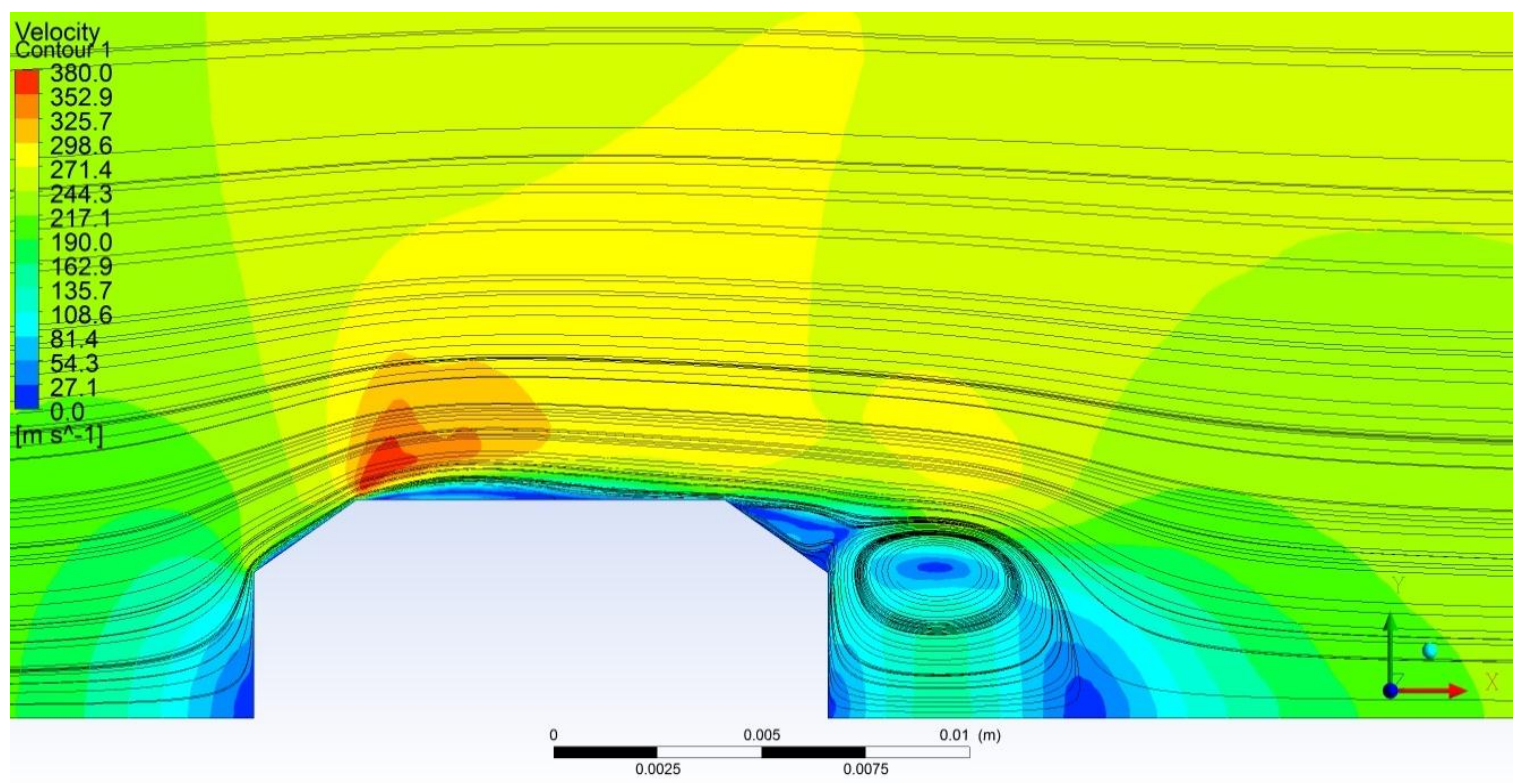

Figure 35. Velocity Contour and Streamlines of 2D, Axisymmetric Swirl Flow over Target at 1.0E-4 s

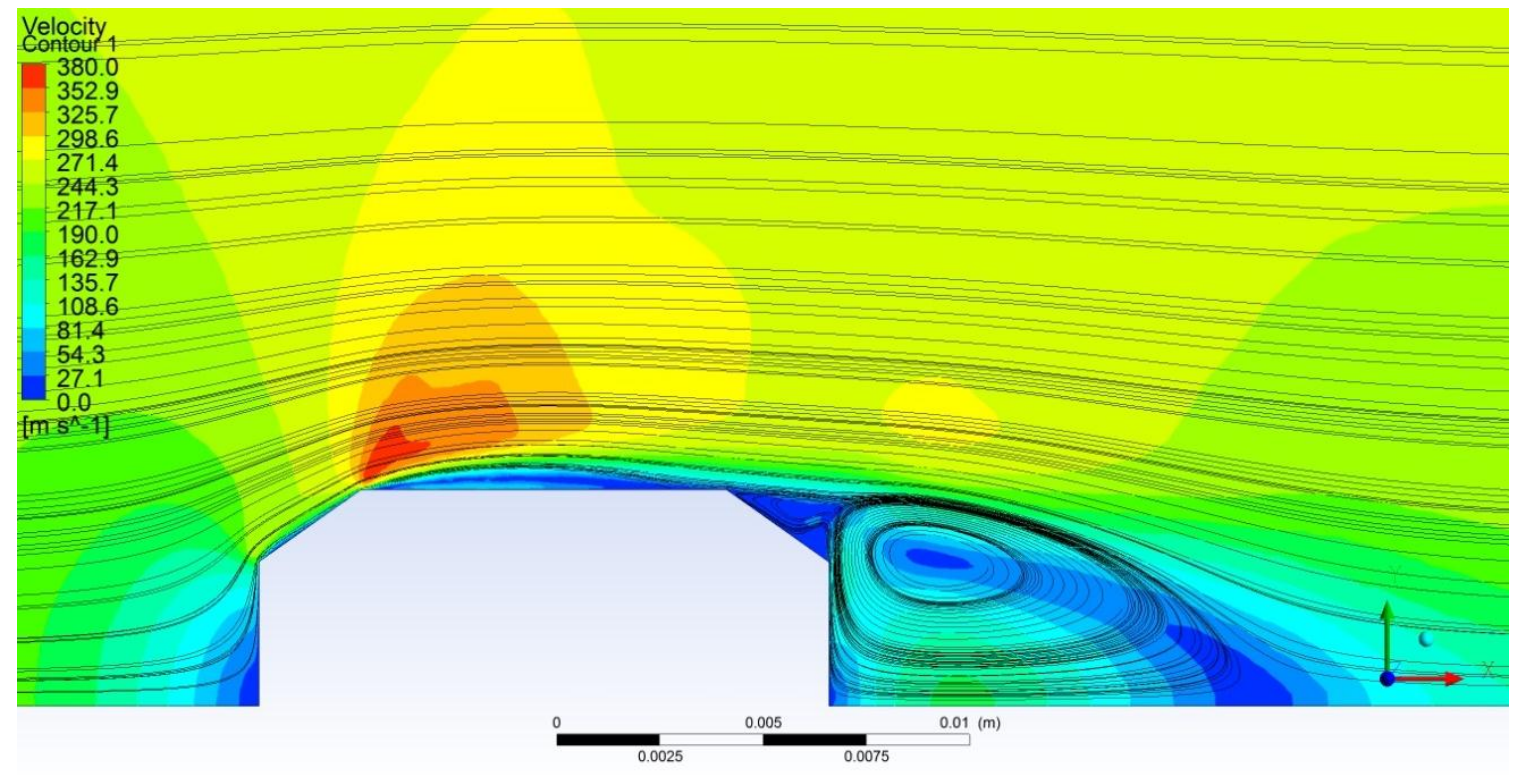

Figure 36. Velocity Contour and Streamlines of 2D, Axisymmetric Swirl Flow over Target at 2.0E-4 s 


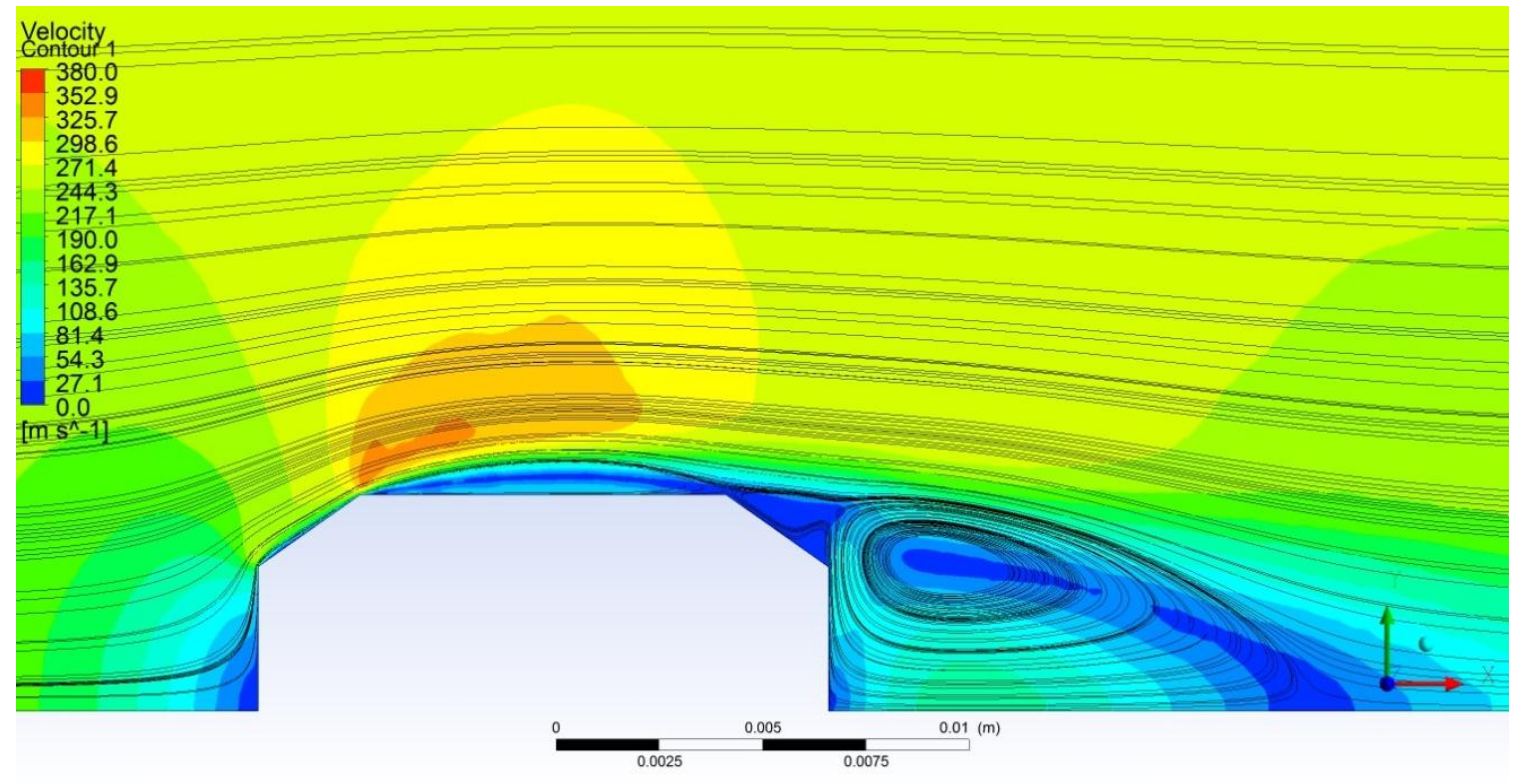

Figure 37. Velocity Contour and Streamlines of 2D, Axisymmetric Swirl Flow over Target at 3.0E-4 s

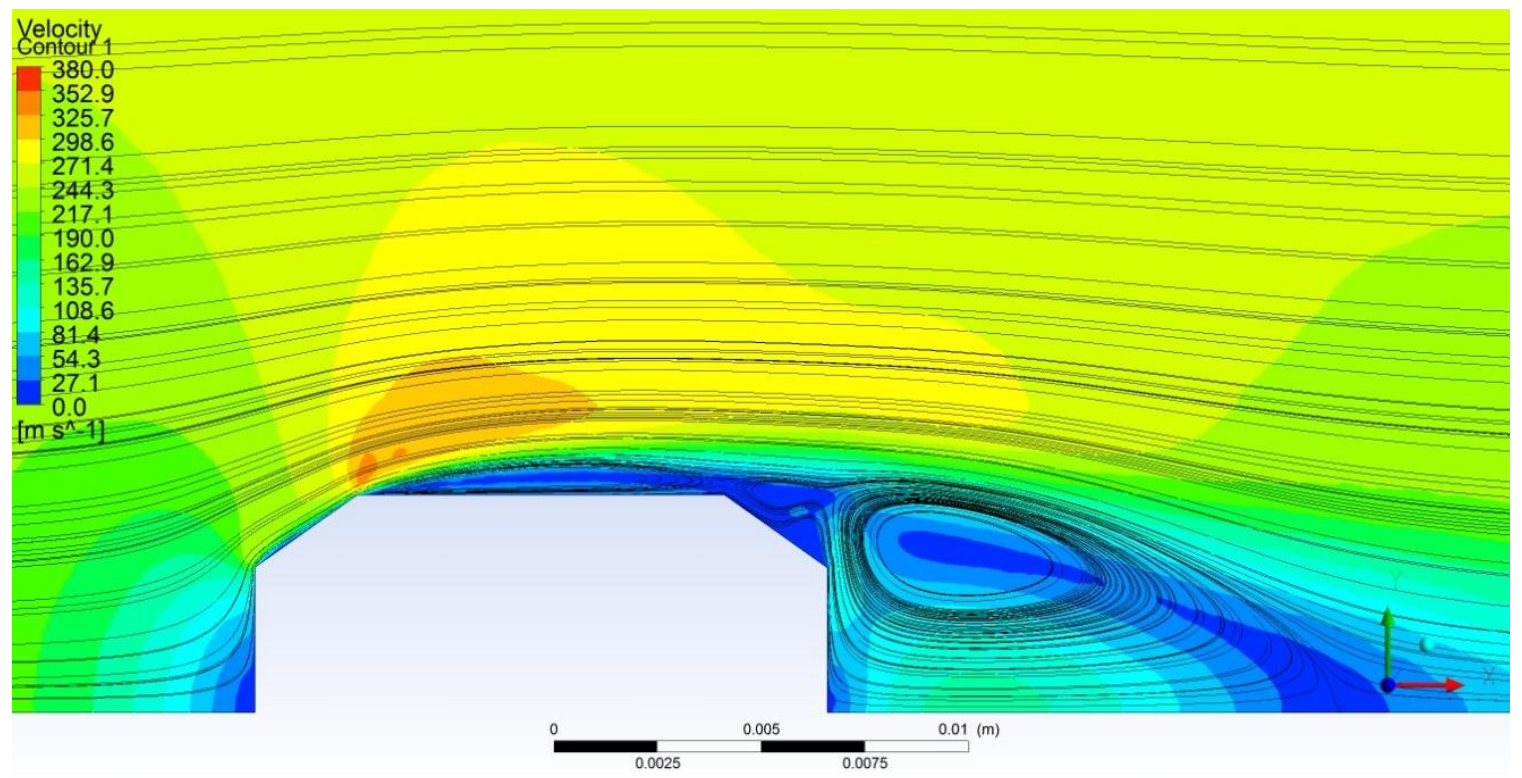

Figure 38. Velocity Contour and Streamlines of 2D, Axisymmetric Swirl Flow over Target at 4.0E-4 s 


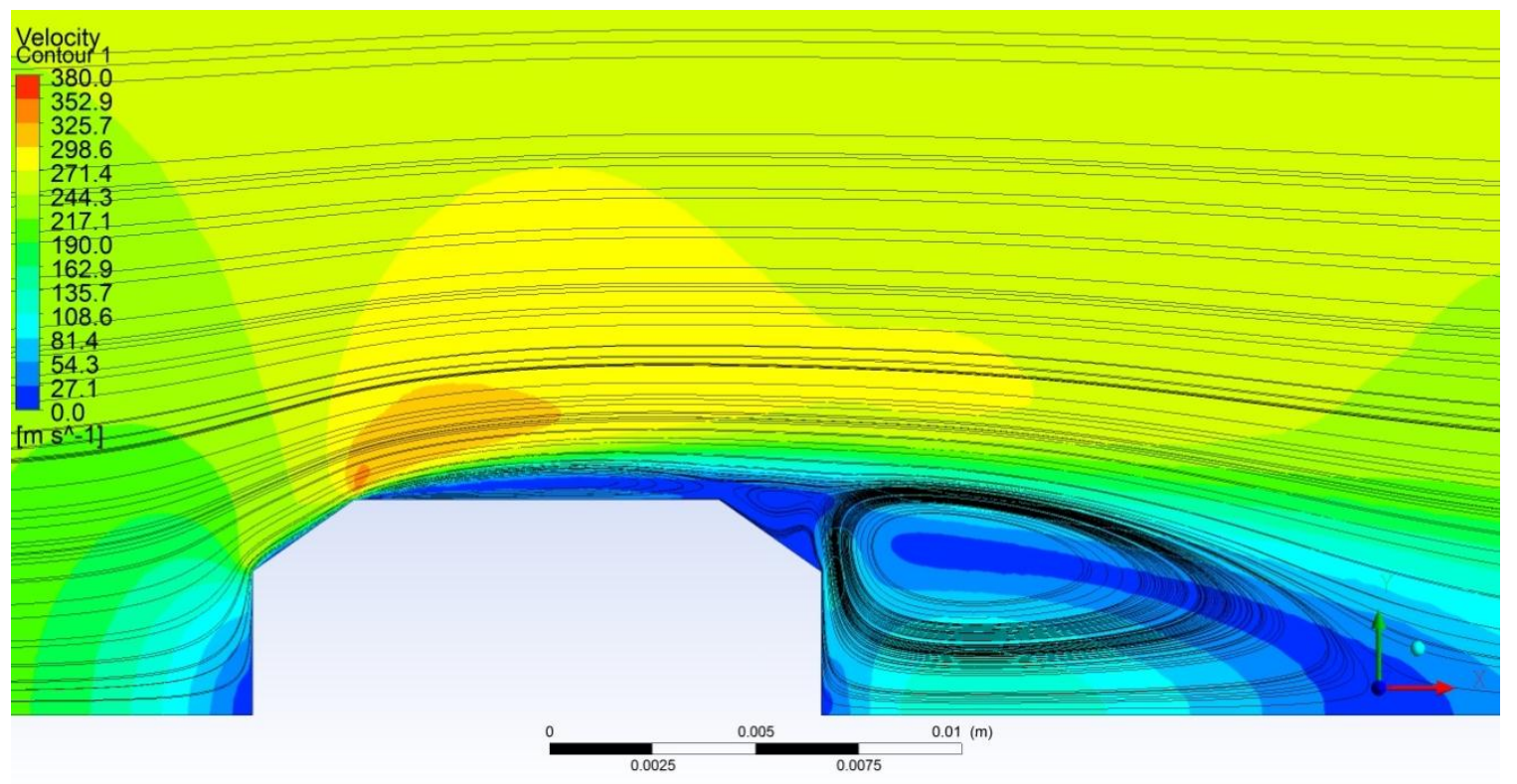

Figure 39. Velocity Contour and Streamlines of 2D, Axisymmetric Swirl Flow over Target at 5.0E-4 s

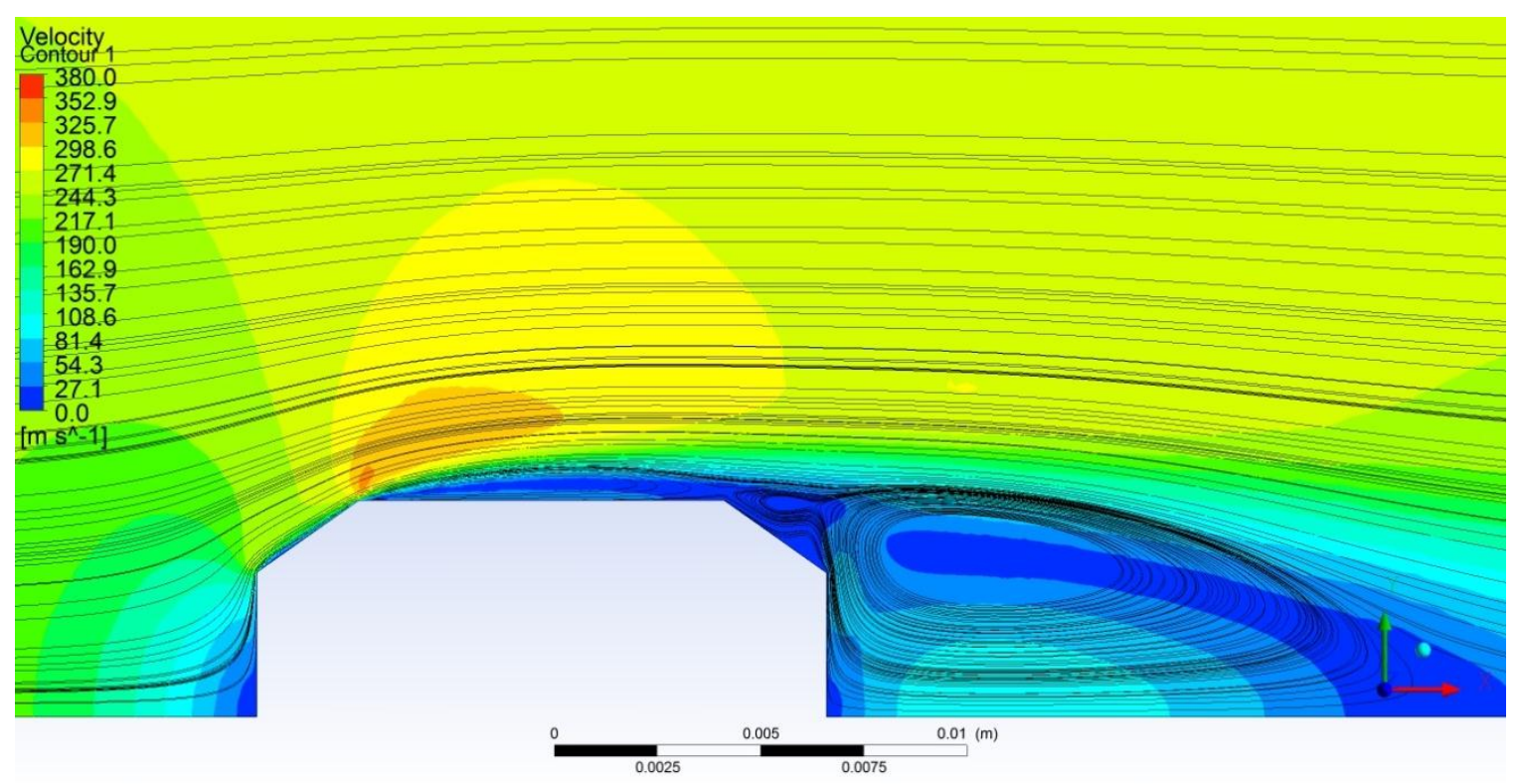

Figure 40. Velocity Contour and Streamlines of 2D, Axisymmetric Swirl Flow over Target at 6.0E-4 s 


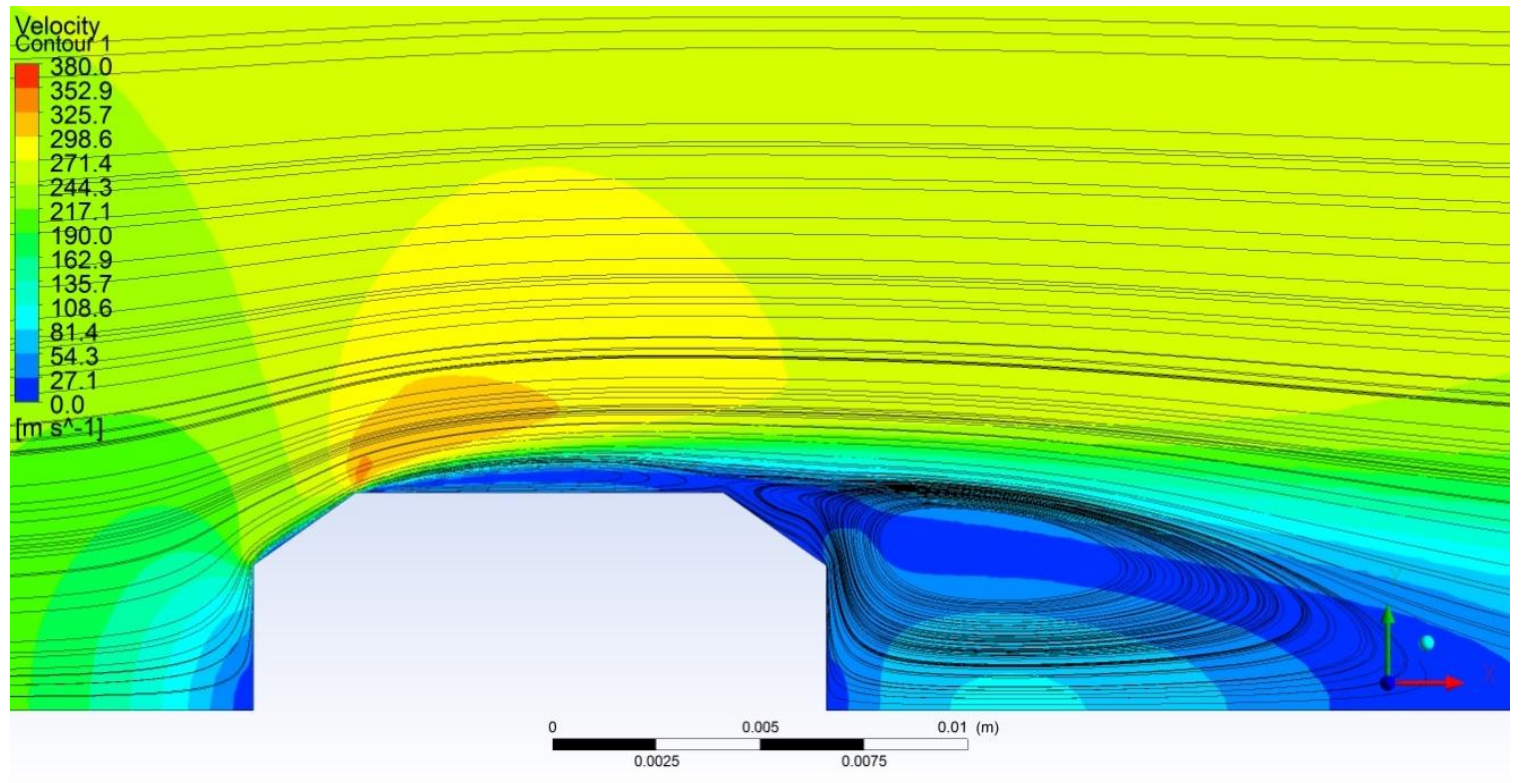

Figure 41. Velocity Contour and Streamlines of 2D, Axisymmetric Swirl Flow over Target at 7.0E-4 s

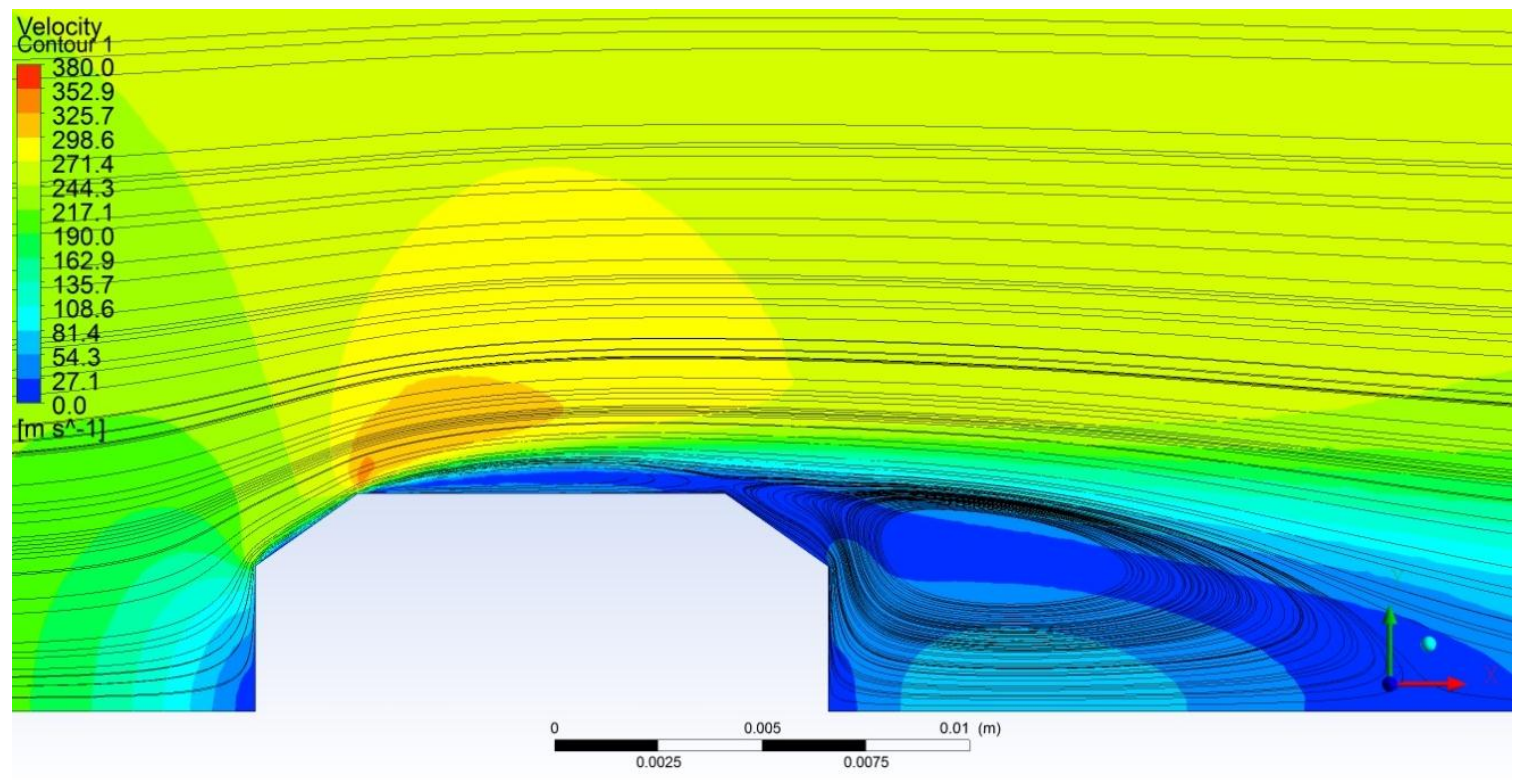

Figure 42. Velocity Contour and Streamlines of 2D, Axisymmetric Swirl Flow over Target at 8.0E-4 s 


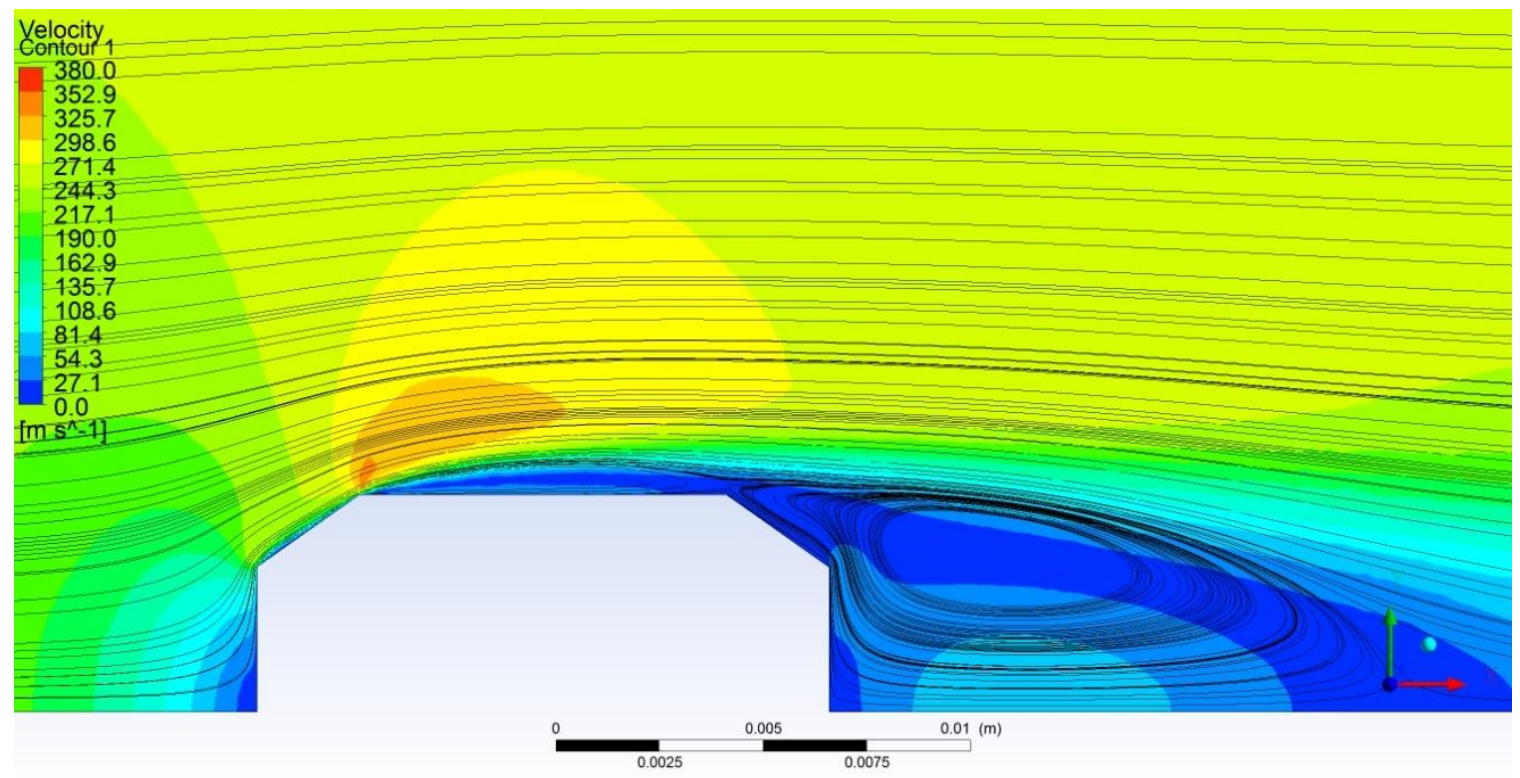

Figure 43. Velocity Contour and Streamlines of 2D, Axisymmetric Swirl Flow over Target at 9.0E-4 s

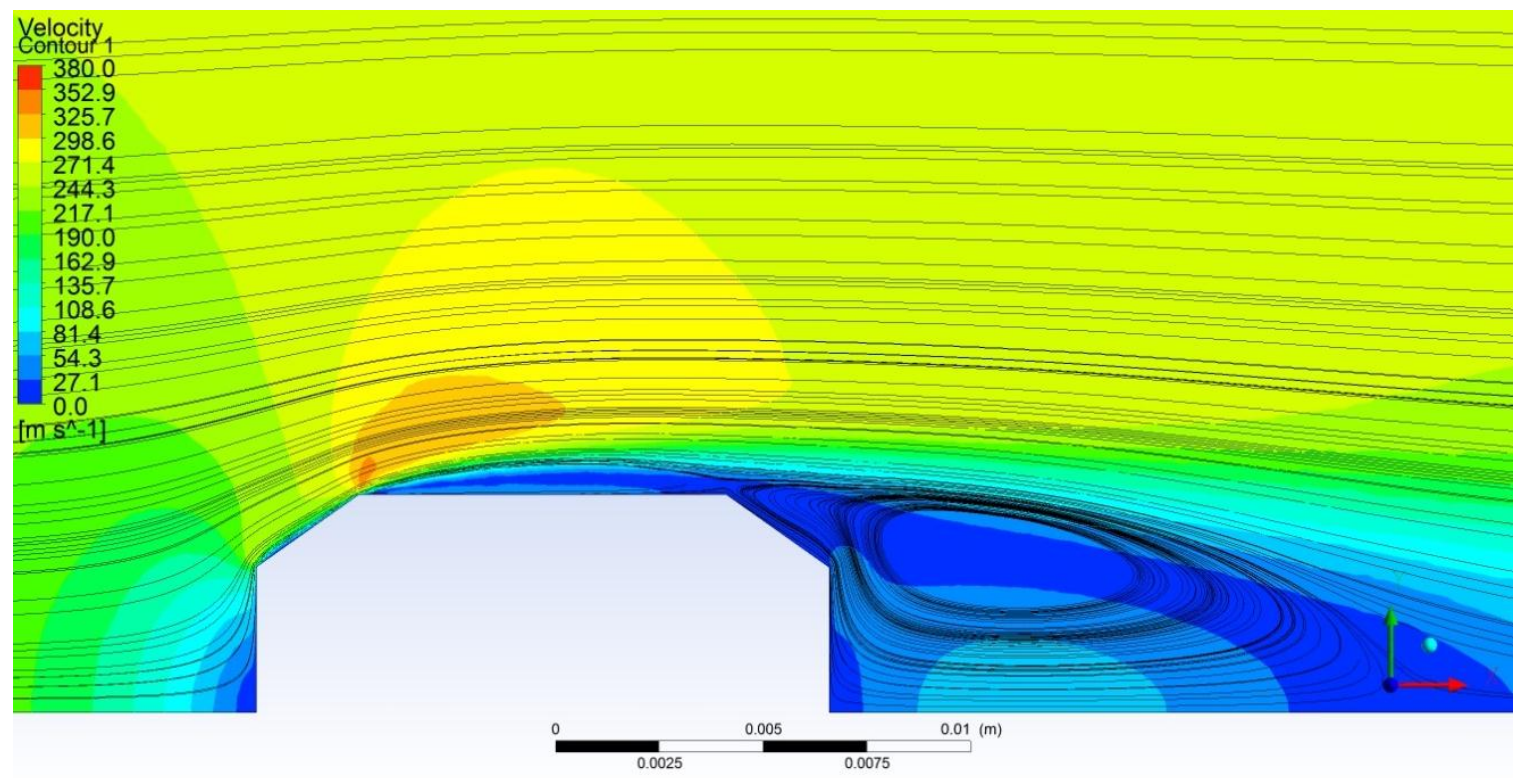

Figure 44. Velocity Contour and Streamlines of 2D, Axisymmetric Swirl Flow over Target at 1.0E-3 s

Although the velocity contour and streamline plots indicate the flow reaches a steady state, the changes in a couple key variables, drag force and heat transfer, were also examined over time. Figures 45 and 46 depict the relationship between drag force and 
heat transfer with time. (Note that the target walls are labeled in order from front to

back.) It appears that both the drag force and heat transfer reach steady values after 8.0E-

$4 \mathrm{~s}$.

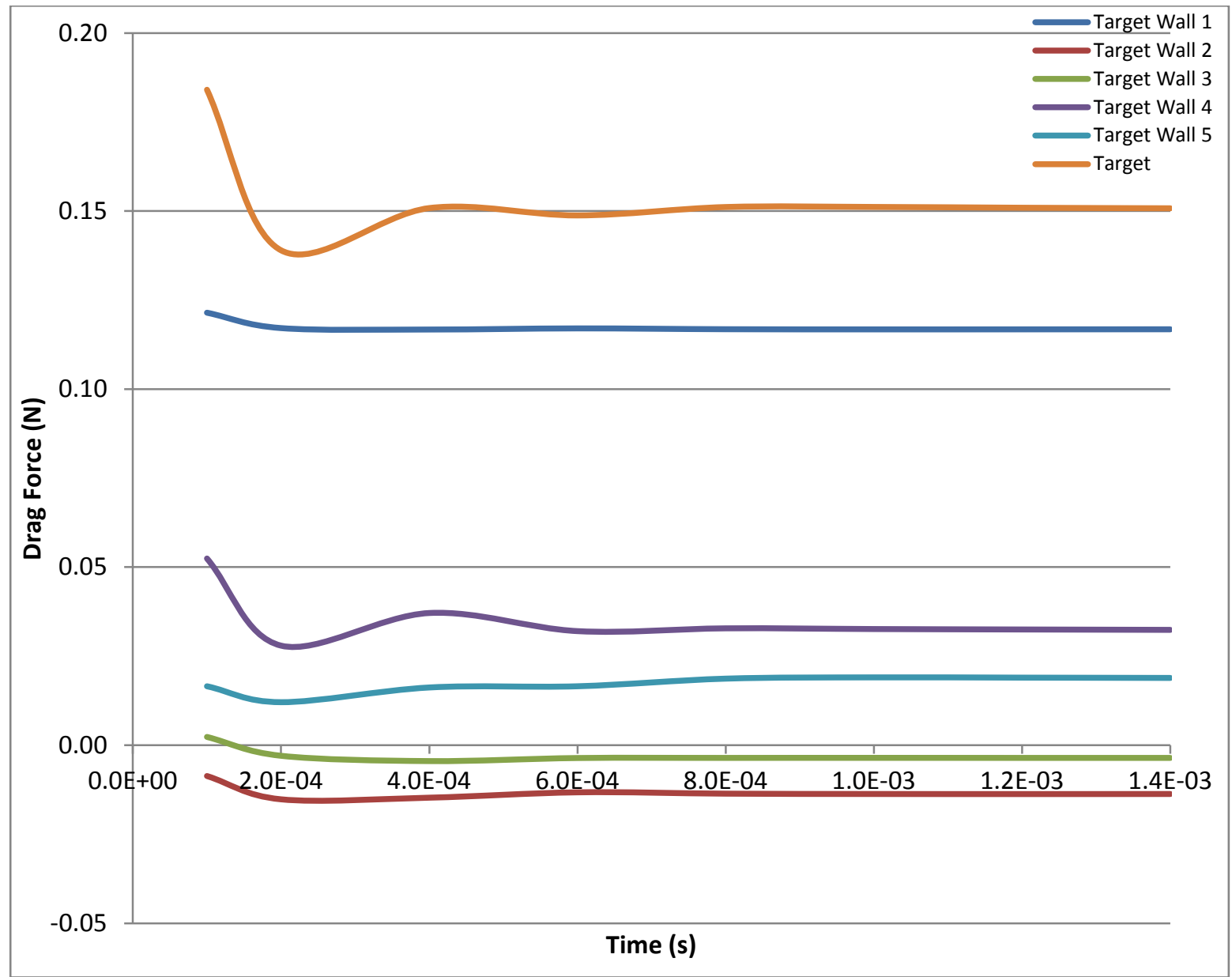

Figure 45. Target Drag Force over Time in Drift Region from 2D, Axisymmetric Swirl Model 


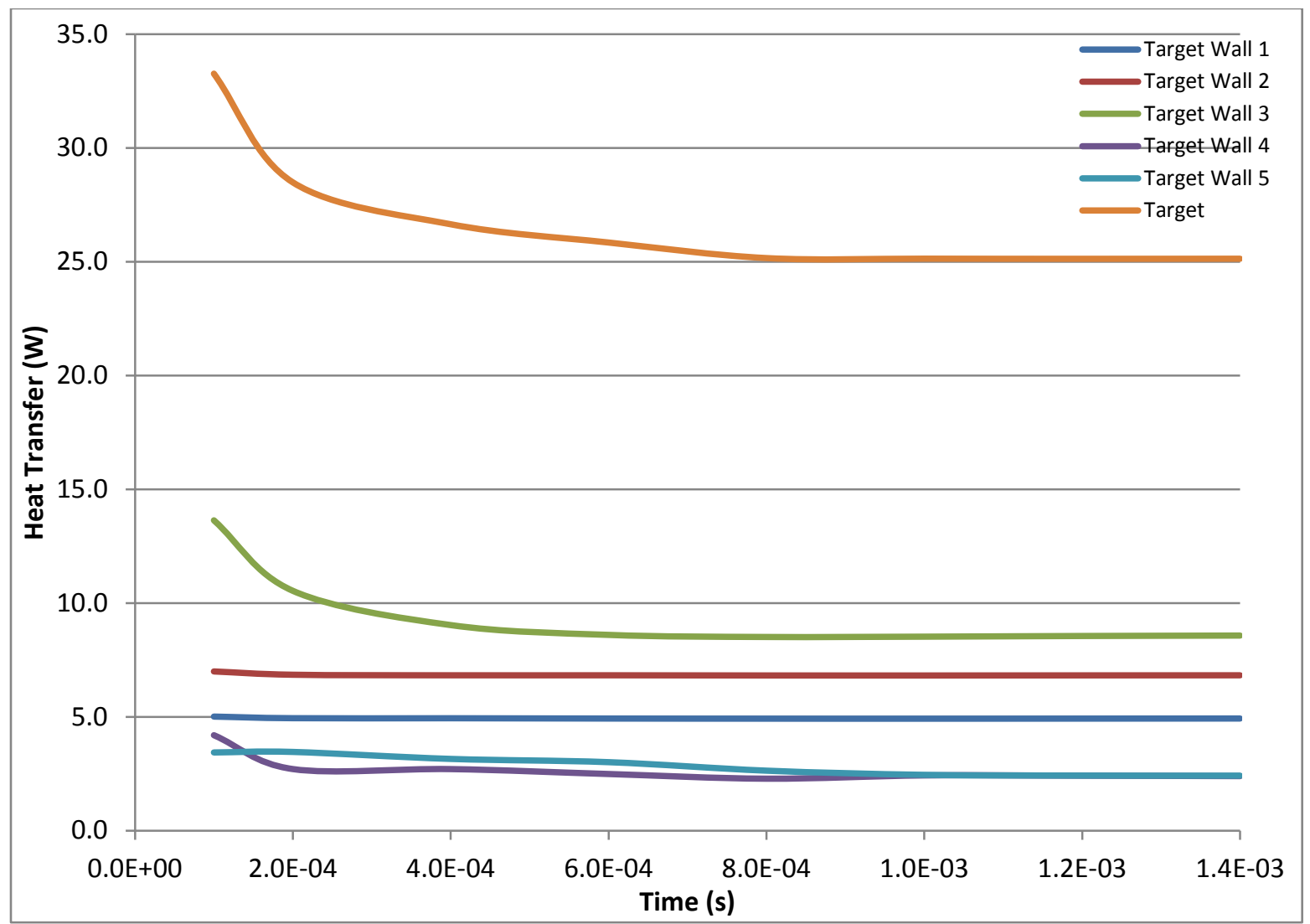

Figure 46. Target Heat Transfer over Time in Drift Region from 2D, Axisymmetric Swirl Model

As seen in Figure 45, the largest contribution to the total drag force is from the front wall. Since this wall acts like a blunt object perpendicular to the flow, a relatively large drag force is to be expected. Also for this reason, the majority of the drag force on wall 1 should come from pressure drag. Wall 5 also acts as a blunt surface in the flow, and the pressure drag should comprise most of the drag from this face. Table 16, which shows the drag force on each wall due to both pressure and viscous forces, confirms these drag predictions. The flow over wall 3, which lies horizontal to the flow, should act like flow over a flat plate parallel to the free-stream flow. The drag force should be due to the viscous forces acting on the surface. Table 16 verifies this prediction as well. Note that the viscous drag is negative along this wall. As can be seen in the streamlines in the 
velocity contour plots discussed previously, a recirculation vortex occurs along this wall. Therefore, the fluid directly above wall 3 flows upstream, giving the drag force a negative value along this wall. Note that the viscous drag force on wall 4, which also encounters reversed flow due to a recirculation vortex, also has a negative viscous drag force. However, the pressure drag force on wall 4 is positive and greater in magnitude than the viscous drag force, thereby making the overall drag force from wall 4 positive. The flow over wall 2 is not part of a recirculation region and, therefore, wall 2 has a positive viscous drag force. However, the positive viscous drag force is outweighed by the negative pressure drag force. Table 16 below presents the drag and heat transfer on each target wall after $1 \mathrm{~ms}$ and was used to compare the drag and heat transfer between the transient, 2D, axisymmetric model and the 3D, steady state one. (See the 3D drift regime results section.)

Table 16. Drag Force and Heat Transfer on Target after 1 ms from 2D, Axisymmetric Swirl Model

\begin{tabular}{|c|c|c|c|c|c|c|}
\hline & $\begin{array}{c}\text { Pressure } \\
\text { Drag } \\
\text { Force (N) }\end{array}$ & $\begin{array}{c}\text { Viscous } \\
\text { Drag } \\
\text { Force (N) }\end{array}$ & $\begin{array}{c}\text { Total } \\
\text { Drag } \\
\text { Force (N) }\end{array}$ & $\begin{array}{c}\text { Drag } \\
\text { Coefficient }\end{array}$ & $\begin{array}{c}\text { Heat } \\
\text { Transfer } \\
(\mathbf{W})\end{array}$ & $\begin{array}{c}\text { Heat Transfer } \\
\text { Coefficient } \\
\left(\mathbf{W} /\left(\mathbf{m}^{2}-\mathbf{K}\right)\right)\end{array}$ \\
\hline Target Wall 1 & 0.1168 & $1.12 \mathrm{E}-17$ & 0.1168 & & 4.9179 & \\
\hline Target Wall 2 & -0.0179 & 0.0042 & -0.0137 & & 6.8164 & \\
\hline Target Wall 3 & 0.0 & -0.0036 & -0.0036 & & 8.5235 & \\
\hline Target Wall 4 & 0.0329 & -0.0003 & 0.0326 & & 2.4259 & \\
\hline Target Wall 5 & 0.0190 & $8.65 \mathrm{E}-25$ & 0.0190 & & 2.4501 & \\
\hline Total Target & 0.1508 & 0.0004 & 0.1512 & 0.5572 & 25.134 & 45.56 \\
\hline
\end{tabular}

As another test of reaching steady state, the absolute pressure, Mach number, turbulent intensity, and heat flux were plotted around the target at different times. Notice that as time elapses, the plots of each variable become more aligned with the plot at the previous time, indicating the flow is reaching a steady state. As seen in the velocity contour plots, the velocity increases around the front of the target. The plot of the Mach 
number, which is a function of the velocity, shows this velocity trend along the first 2 target walls in Figure 47. (Note that discontinuities in the plots naturally occur at points of sharp geometry changes.) Also notice the decrease in the Mach number on the back portion of the target, indicating a decrease in velocity behind the object. Recall that this velocity trend aligns well with that for incompressible flow around a sphere [20]. Figure 48 depicts a decreasing (favorable) pressure gradient along the front walls and an increasing (adverse) pressure gradient on the back of the target. This agrees with the pressure trend predicted by Bernoulli's equation for incompressible flow over a sphere [20], indicating the flow over the target may not experience a drastic change in density. Also, note that the greatest target pressure in Figure 48 occurs at the front stagnation point, which is physically reasonable. Additionally, Figure 49 depicts the low turbulent intensity of the flow around the target, which is realistic due to the low Reynolds number of the drift region. The heat flux was also plotted in Figure 50, and further discussion can be found in the 3D drift results section. Other variables were plotted at these times and placed in Appendix B. See Figures 71-75. 


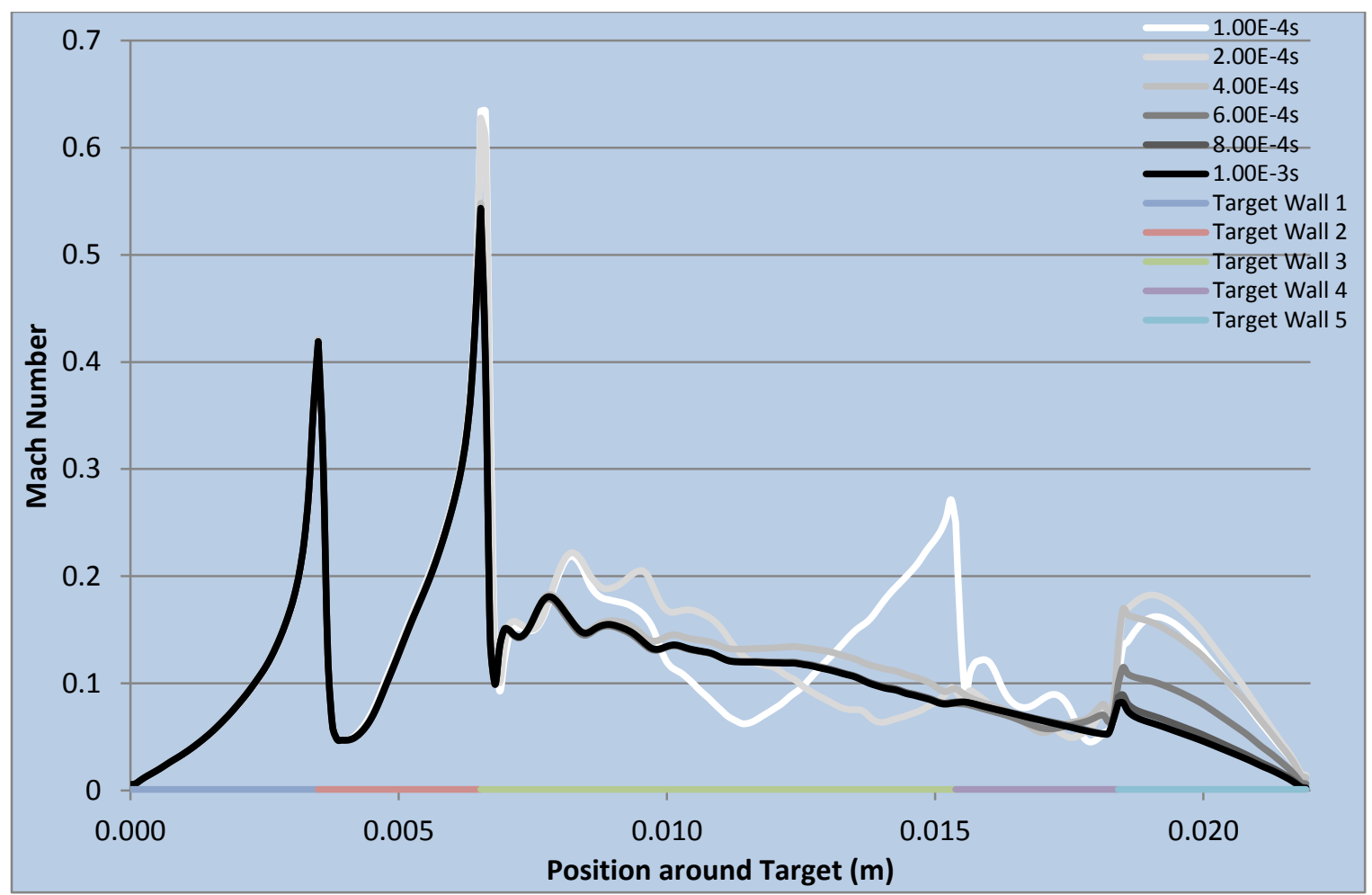

Figure 47. Mach Number around Target in Drift Region from 2D, Axisymmetric Swirl Model

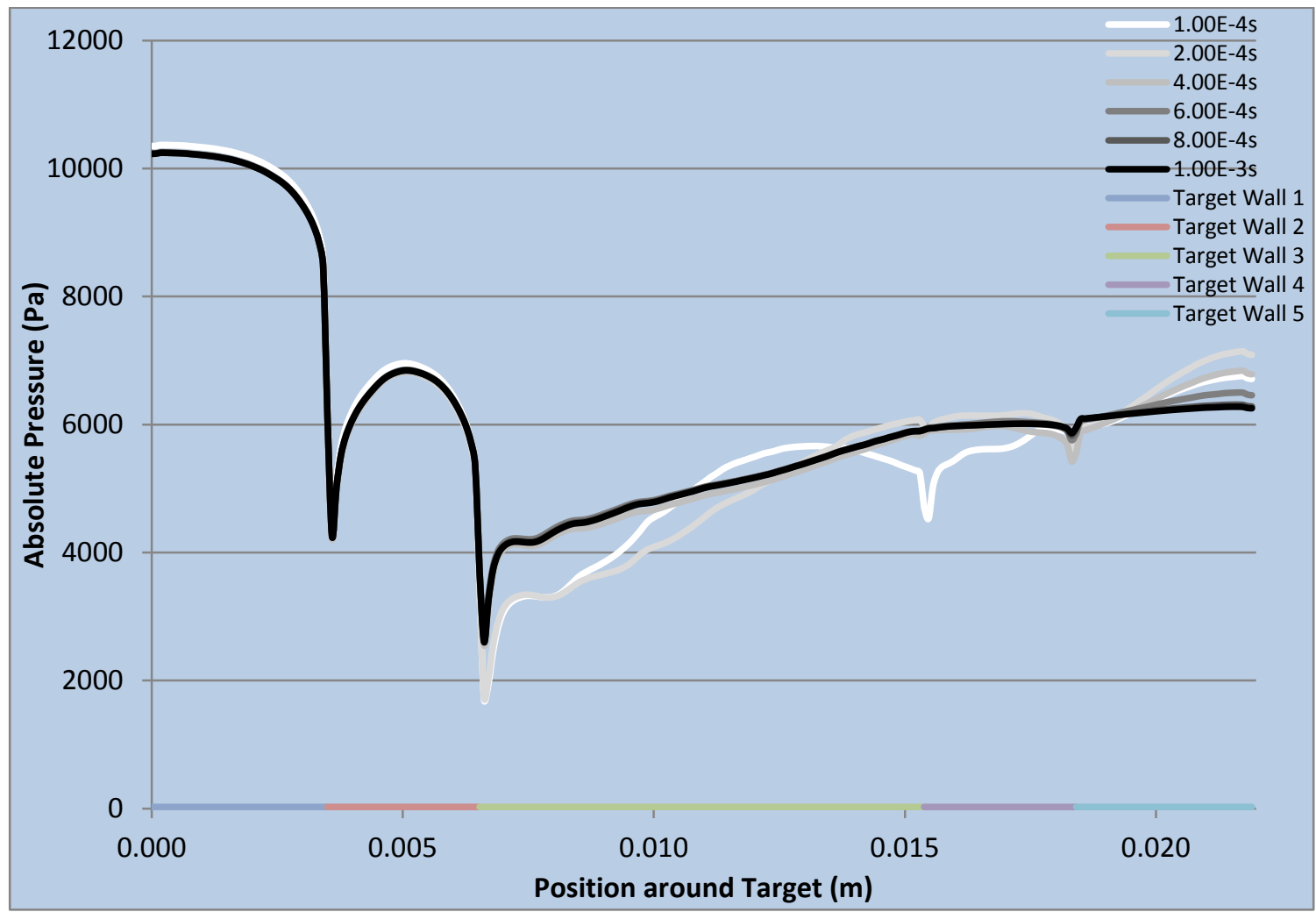

Figure 48. Absolute Pressure around Target in Drift Region from 2D, Axisymmetric Swirl Model 


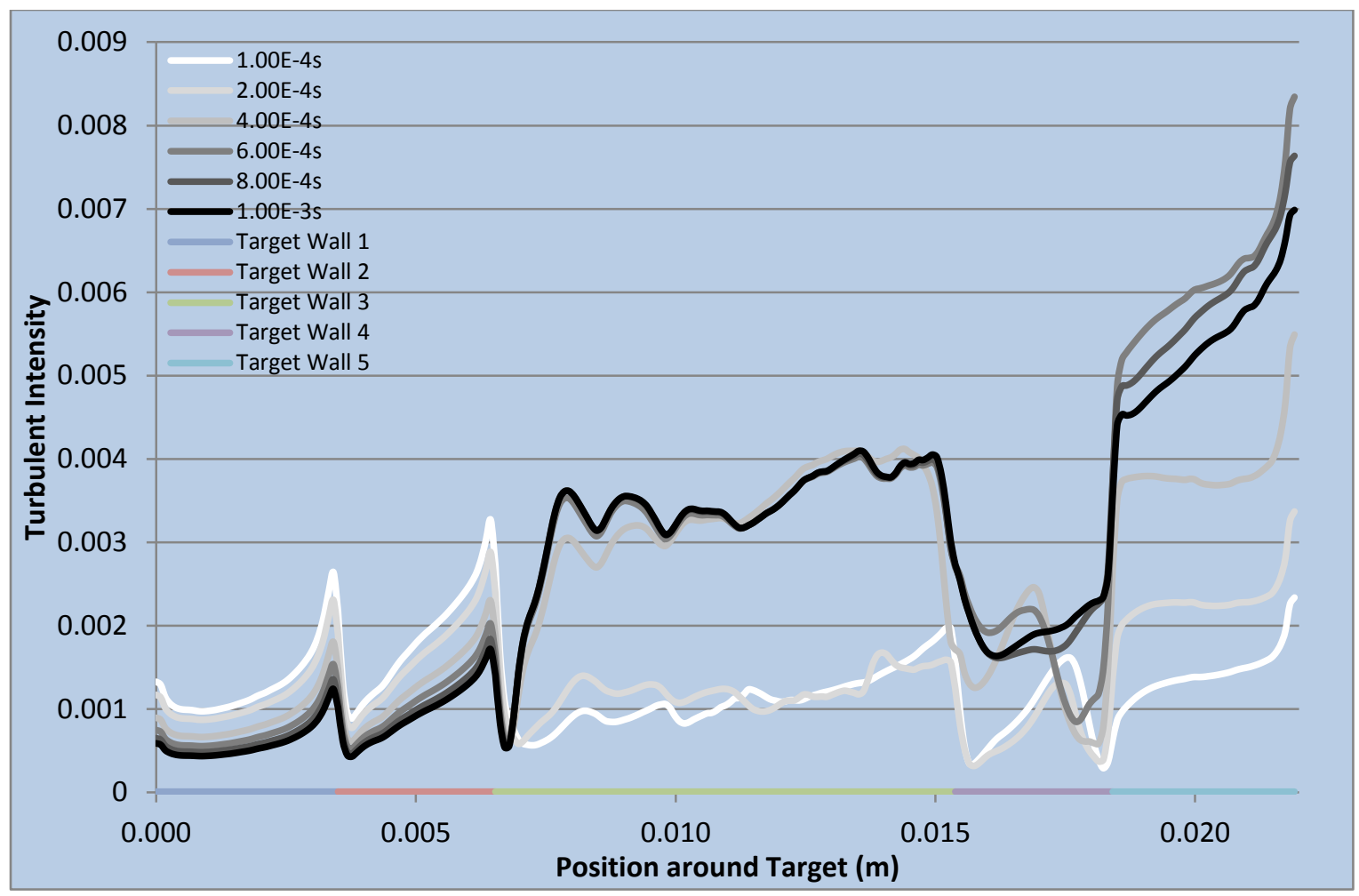

Figure 49. Turbulent Intensity around Target in Drift Region from 2D, Axisymmetric Swirl Model

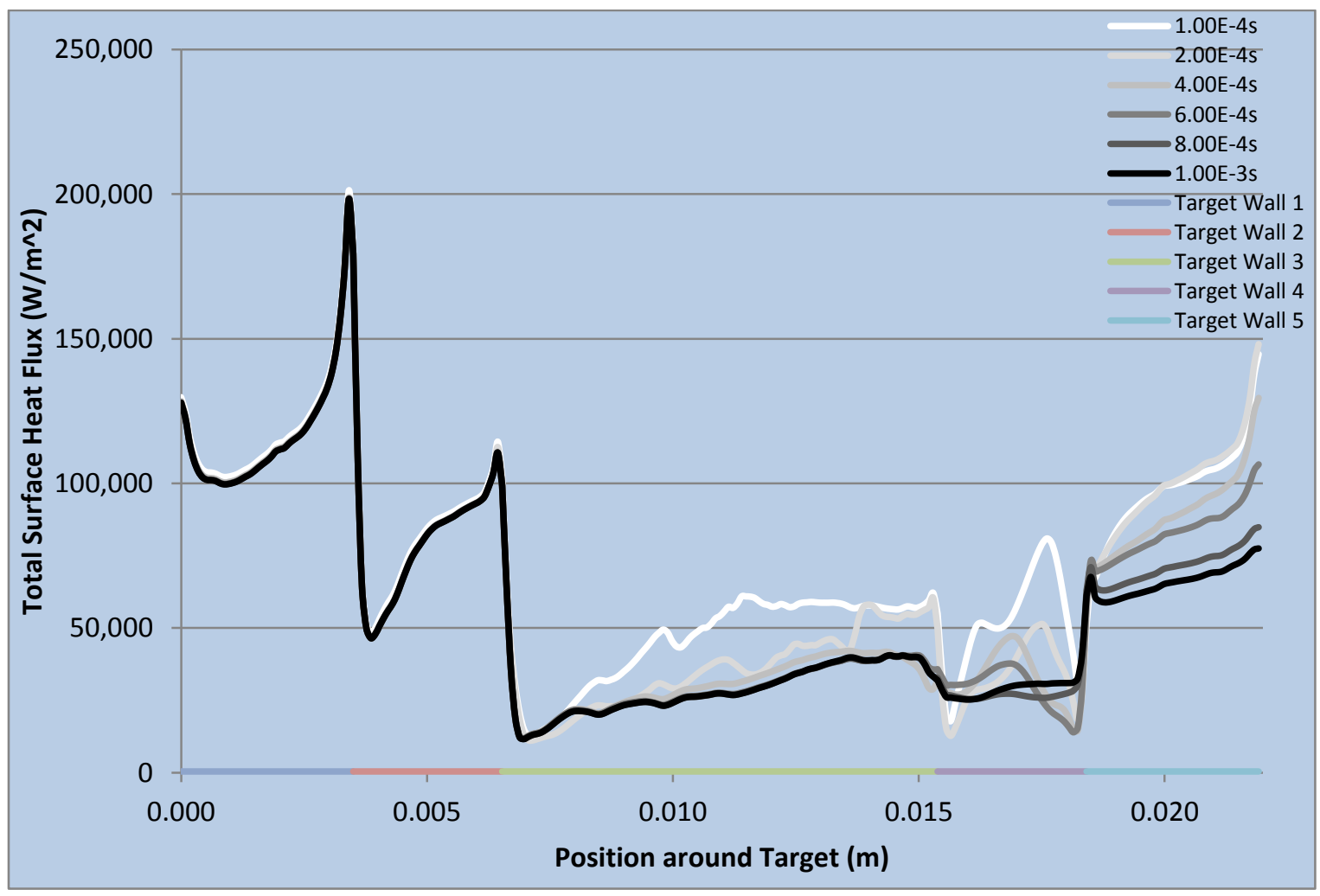

Figure 50. Total Surface Heat Flux around Target in Drift Region from 2D, Axisymmetric Swirl Model 
Note that the $y+$ values for the $2 \mathrm{D}$, axisymmetric swirl simulation were approximately between 0.5 and 3.25. (See Figure 75 in Appendix B for the $y+$ plot.) The SST k-omega turbulence model uses enhanced wall treatment as a default, which is relatively insensitive to $\mathrm{y}+$ values [1]. However, for greatest accuracy, $\mathrm{y}+$ values should be on the order of 1 . This confirms reasonable mesh refinement at the target wall. 


\section{D Drift Regime: Results}

Since the 2D, axisymmetric swirl model of the flow around the target in the drift region indicated the existence of a steady-state solution, the 3D model was set up as a steady-state simulation. The results of the 3D, steady-state model of a target in the drift region as described in the $3 \mathrm{D}$ drift regime section in the CFD chapter are presented in this section. A 5,450,161-node mesh consisting of 12 inflation layers with a 1.10 growth rate and a default transition ratio of 0.272 , an overall growth rate of 1.03 , and a target edge size of 1E-4 m was created from an axisymmetric domain spanning 20 body lengths before, 15 body lengths above, and 20 body lengths behind. The Fluent case setup can be seen in Table 24 in Appendix A.

A velocity contour and streamline plot was made on the $x-y$ plane with the $3 \mathrm{D}$ results. It can be seen in Figure 51. Notice that this looks very similar to the velocity contour and streamline plot from the 2D, axisymmetric swirl model at $1 \mathrm{~ms}$ in Figure 44. Figure 51 indicates the flow around the target is axisymmetric and can be simulated with a 2D, axisymmetric swirl model. The pressure and temperature contours also look similar to those of the 2D, axisymmetric swirl model and can be seen in Appendix $\mathrm{C}$ in Figures 76 and 77. 


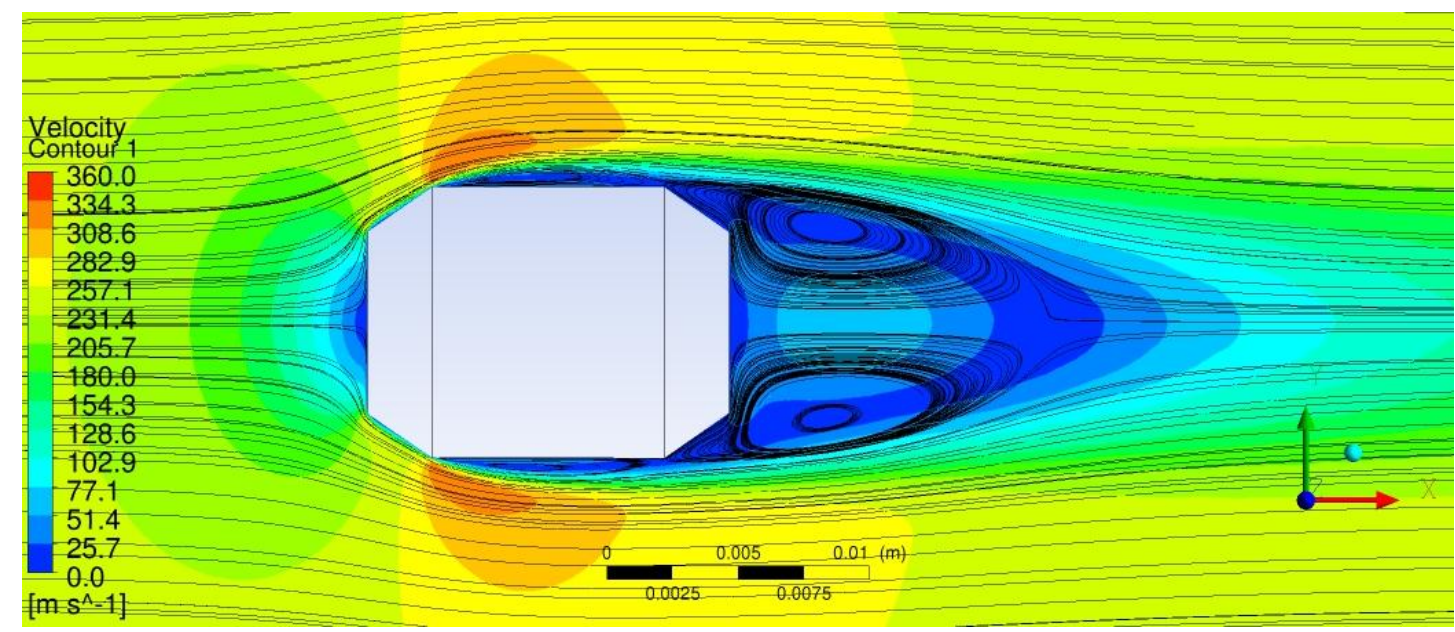

Figure 51. Velocity Contour and Streamline Plot for Target in 3D, Steady-State, Drift Simulation

As with the $2 \mathrm{D}$, axisymmetric swirl simulation, several variables were plotted around the target. The absolute pressure, Mach number, turbulent intensity, and heat flux can be seen in Figures 52-55. Others can be seen in Figures 78-82 in Appendix C.

Comparison between these plots and the 2D, axisymmetric swirl model plots at $1 \mathrm{~ms}$ are quite close. This serves as another confirmation of the 2D, axisymmetric swirl model of the flow around the target. 


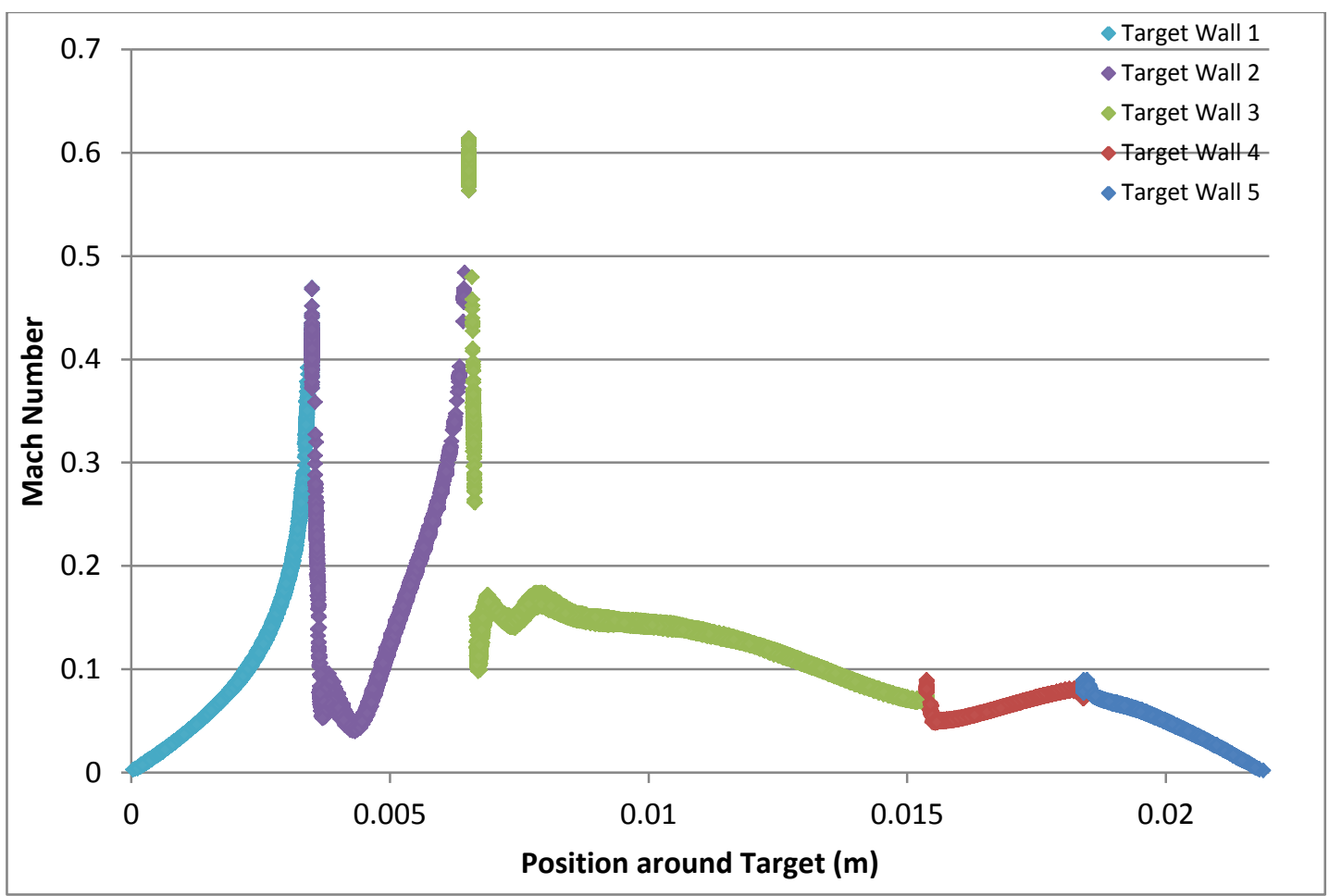

Figure 52. Mach Number around Target from 3D, Steady-State Simulation

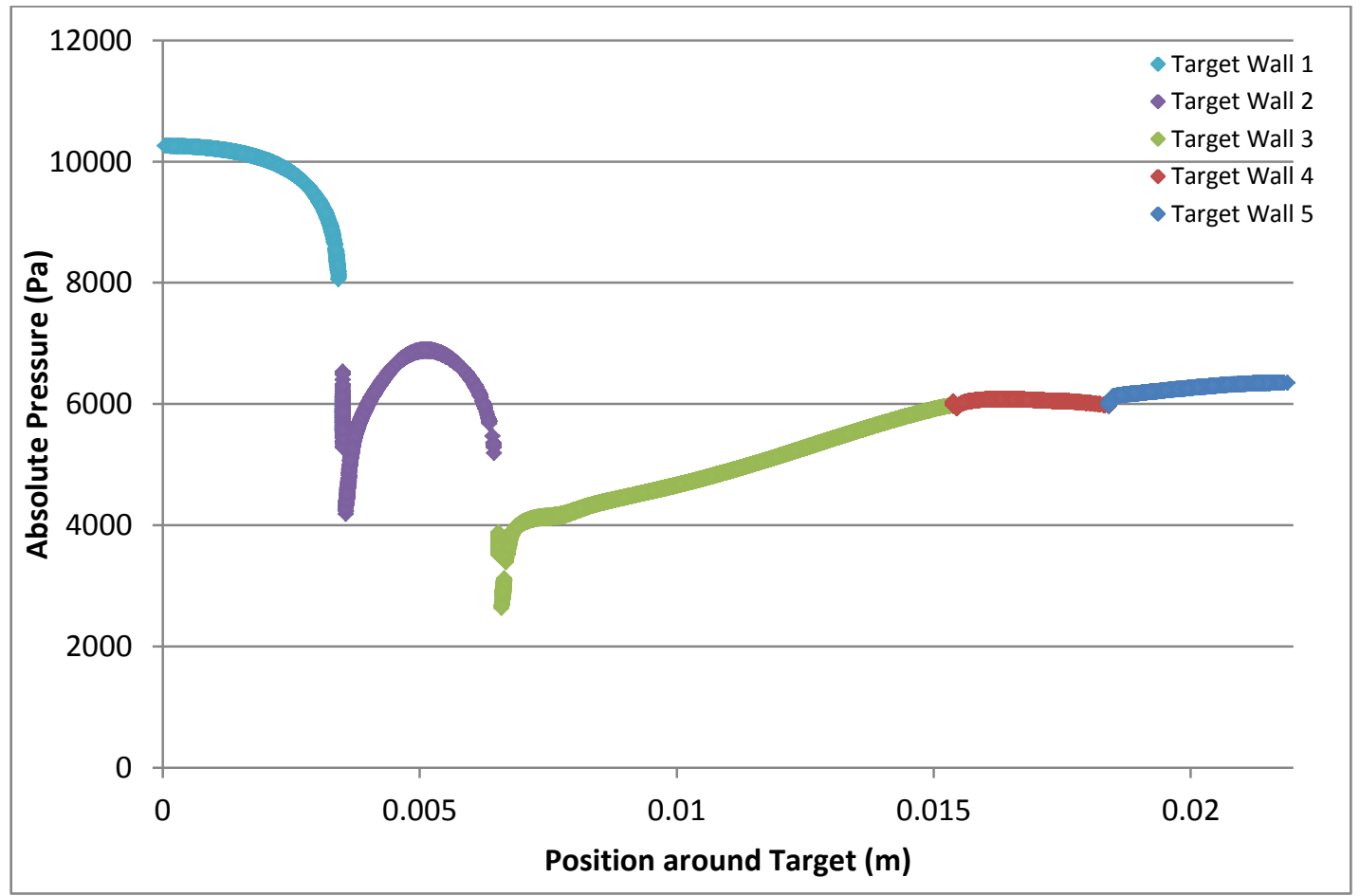

Figure 53. Absolute Pressure around Target from 3D, Steady-State Simulation 


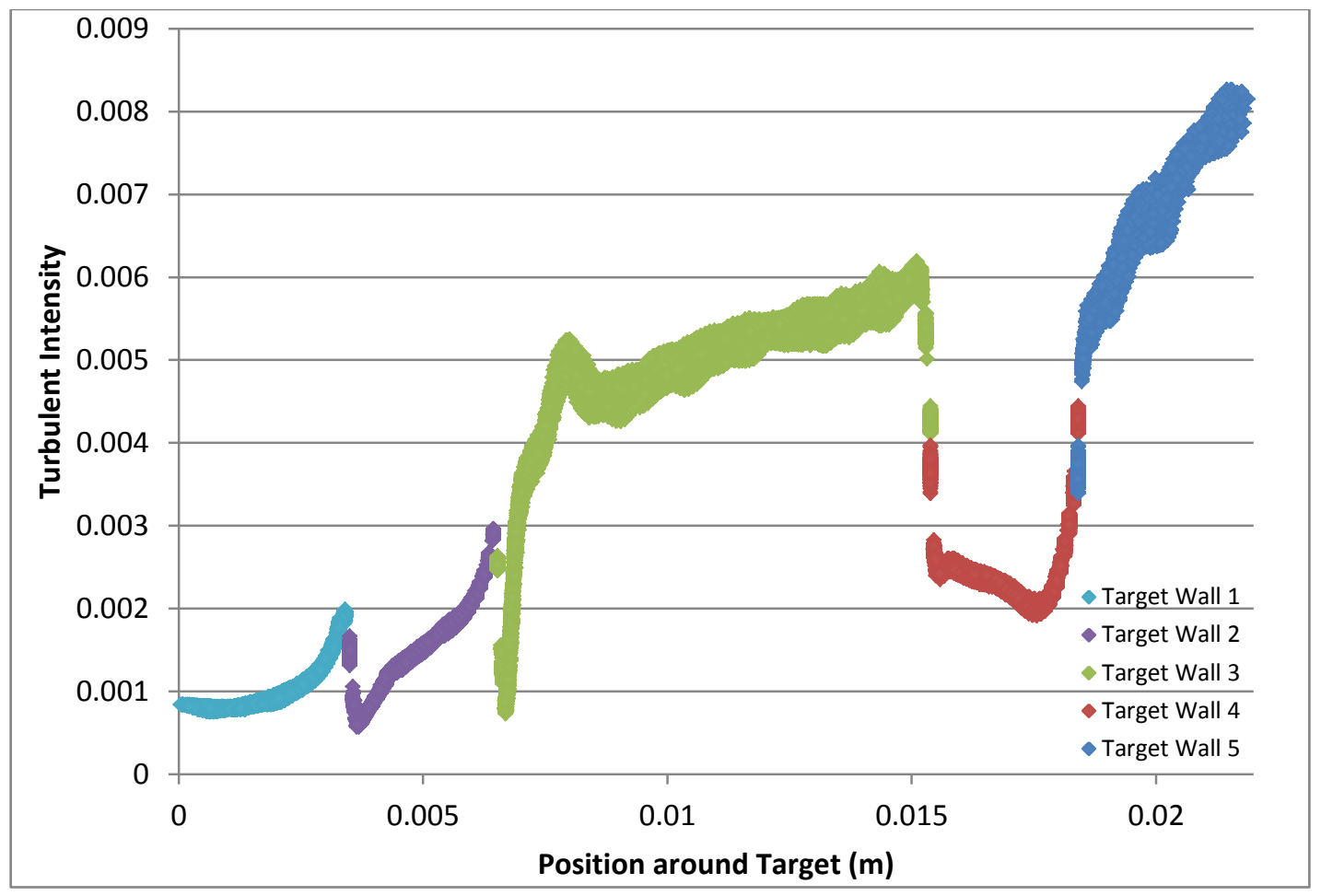

Figure 54. Turbulent Intensity around Target from 3D, Steady-State Simulation

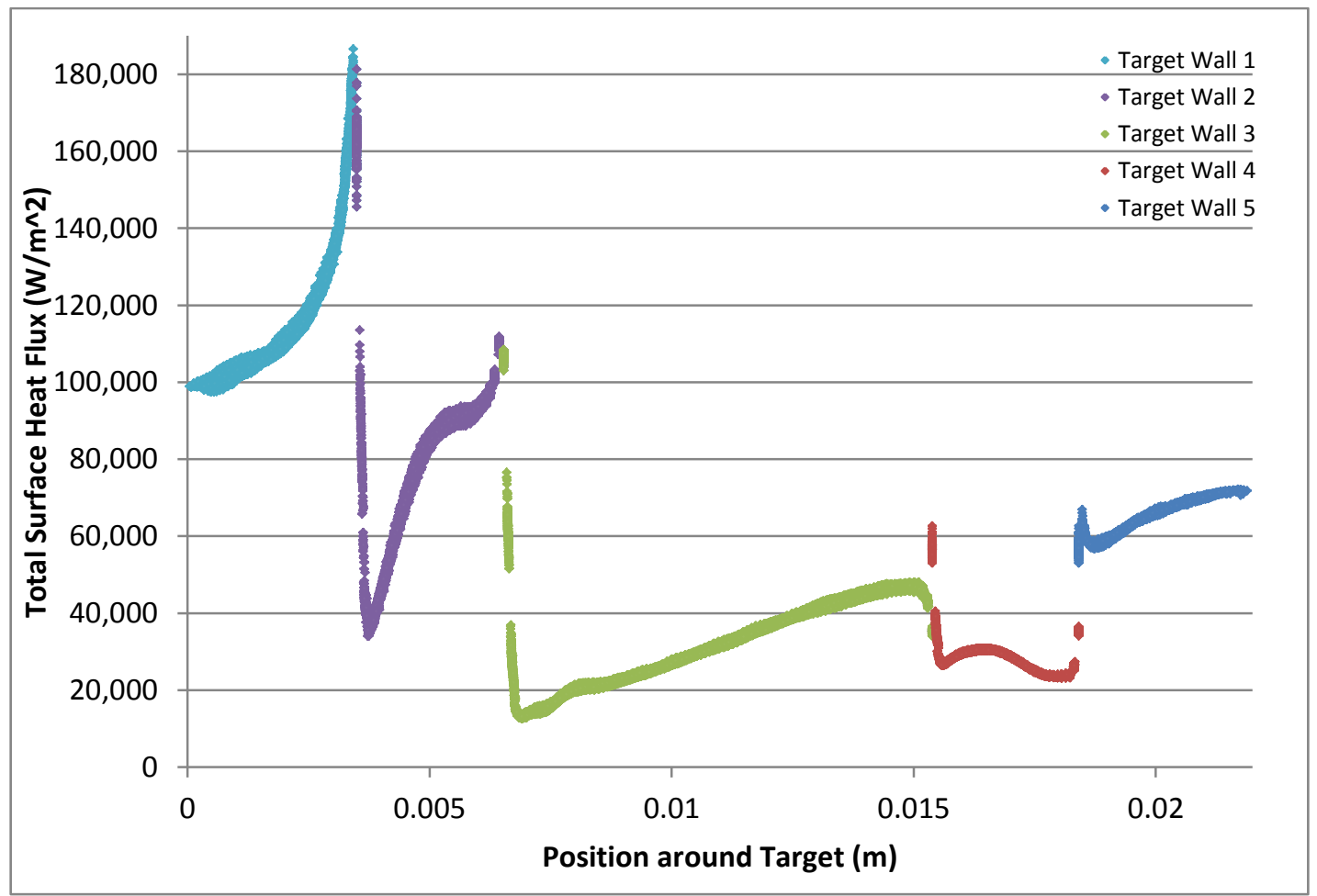

Figure 55. Total Surface Heat Flux around Target from 3D, Steady-State Simulation 
The drag force and heat transfer table from the 2D, axisymmetric swirl results section was also created for the 3D, steady-state results. See Table 17 below. Notice the same trends regarding the drag force are present here. The viscous drag force on the blunt surfaces (walls 1 and 5) and pressure drag force on the horizontal surface (wall 3) are practically zero. The viscous drag force on wall 2 is positive, while the viscous drag force on walls 3 and 4 (where the flow adjacent to the walls is in the reverse direction) is negative. The pressure drag forces outweigh the viscous drag forces on walls 2 and 4.

Table 17. Drag Force and Heat Transfer on Target from 3D, Steady-State Target Model

\begin{tabular}{|c|c|c|c|c|c|c|}
\hline & $\begin{array}{c}\text { Pressure } \\
\text { Drag Force } \\
(\mathbf{N})\end{array}$ & $\begin{array}{c}\text { Viscous } \\
\text { Drag } \\
\text { Force (N) }\end{array}$ & $\begin{array}{c}\text { Total } \\
\text { Drag } \\
\text { Force (N) }\end{array}$ & $\begin{array}{c}\text { Drag } \\
\text { Coefficient }\end{array}$ & $\begin{array}{c}\text { Heat } \\
\text { Transfer } \\
(\mathbf{W})\end{array}$ & $\begin{array}{c}\text { Heat Transfer } \\
\text { Coefficient } \\
\left(\mathbf{W} /\left(\mathbf{m}^{2}-\mathbf{K}\right)\right)\end{array}$ \\
\hline Target Wall 1 & 0.11562 & $-4.321 \mathrm{E}-17$ & 0.11562 & & 4.8120 & \\
\hline Target Wall 2 & -0.01709 & 0.00388 & -0.01321 & & 6.7545 & \\
\hline Target Wall 3 & $-7.161 \mathrm{E}-06$ & -0.00374 & -0.00375 & & 9.4758 & \\
\hline Target Wall 4 & 0.02995 & -0.00026 & 0.02969 & & 2.3011 & \\
\hline Target Wall 5 & 0.01688 & $7.872 \mathrm{E}-18$ & 0.01688 & & 2.4449 & \\
\hline Total Target & 0.14535 & -0.00012 & 0.14523 & 0.5354 & 25.788 & 46.75 \\
\hline
\end{tabular}

Figures 56 and 57 plot the drag coefficient and heat transfer coefficient from both the transient, 2D, axisymmetric swirl and the 3D, steady-state models over time. Good agreement can be seen after $8.0 \mathrm{E}-4 \mathrm{~s}$. Table 18 shows the percent difference between the 3D, steady-state value and the transient, 2D, axisymmetric swirl values. After 8.0E-4 s, the drag and heat transfer coefficients from both models are within $5 \%$ of each other, providing further validation of the $2 \mathrm{D}$, axisymmetric swirl model. 


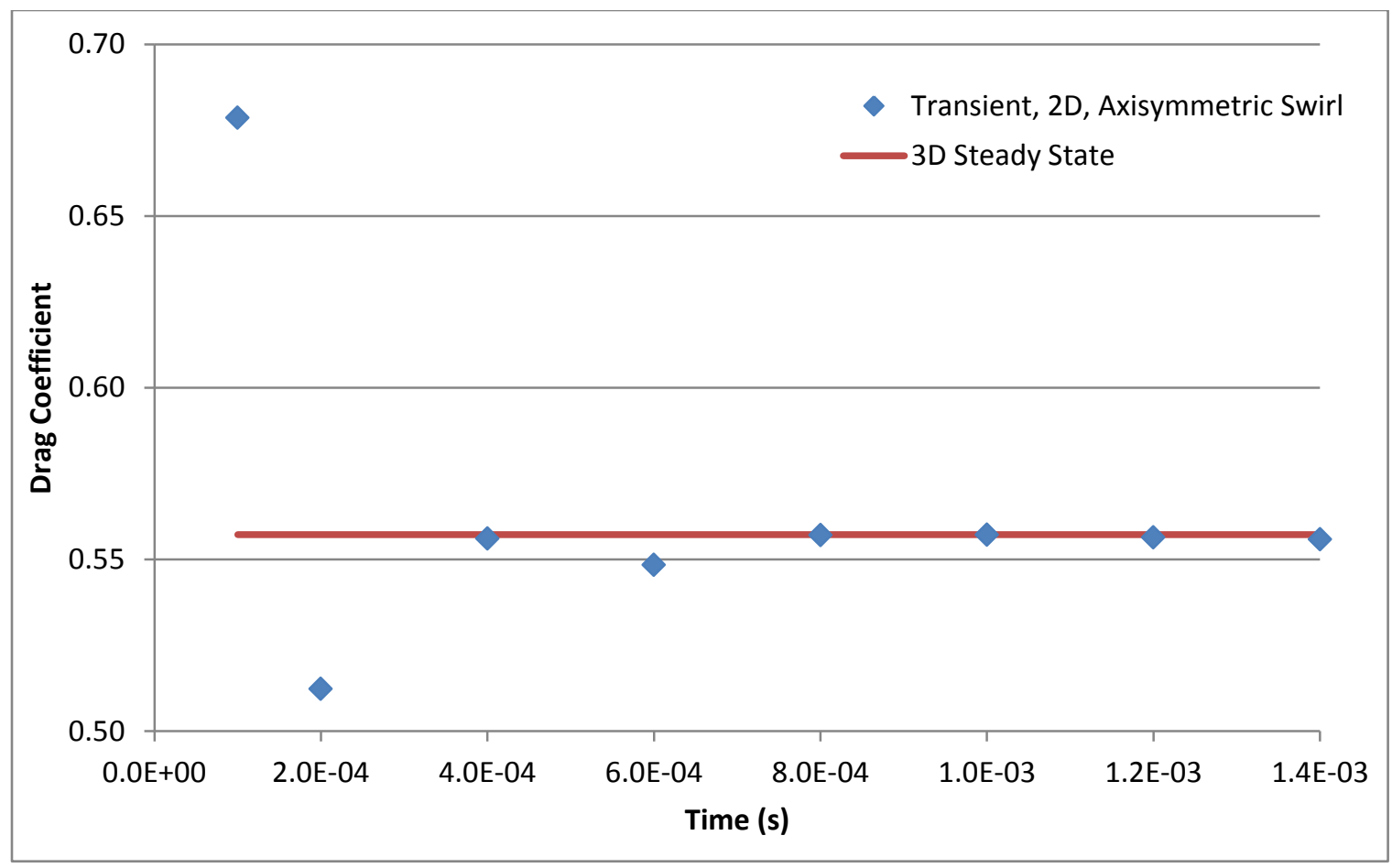

Figure 56. Drag Coefficient Comparison between Transient, 2D, Axisymmetric Swirl Model and 3D, Steady-State Model

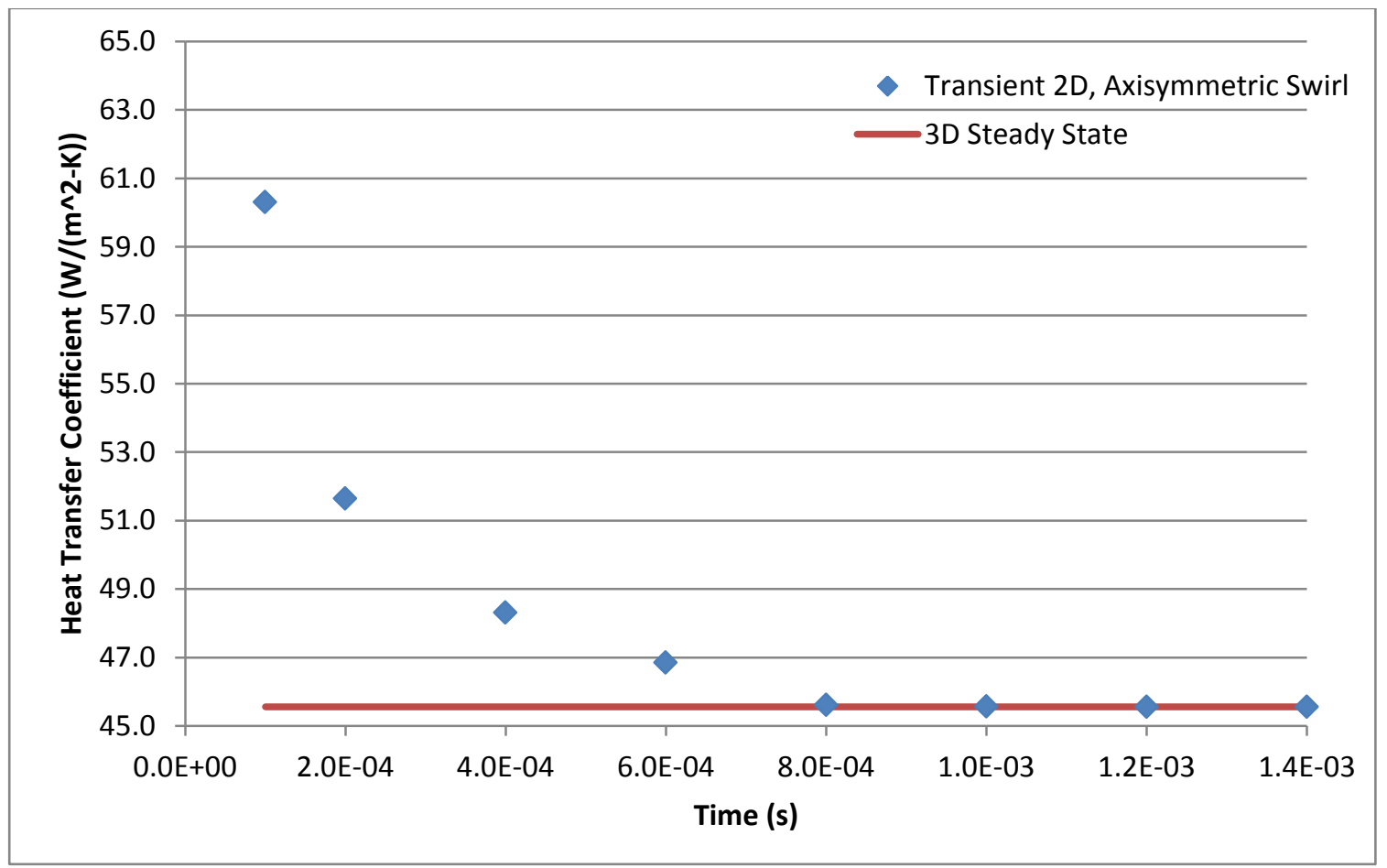

Figure 57. Heat Transfer Coefficient Comparison between Transient, 2D, Axisymmetric Swirl Model and 3D, Steady-State Model 
Table 18. Drag and Heat Transfer Coefficient Percent Difference between Transient, 2D, Axisymmetric Swirl Model and 3D, Steady-State Model

\begin{tabular}{|c|c|c|c|c|}
\hline Time (s) & $\begin{array}{c}\text { Drag } \\
\text { Coefficient }\end{array}$ & $\begin{array}{c}\text { Drag \% } \\
\text { Difference with } \\
\text { 3D Steady-State }\end{array}$ & $\begin{array}{c}\text { Heat Transfer } \\
\text { Coefficient } \\
\left(\mathbf{W} /\left(\mathbf{m}^{2}-\mathbf{K}\right)\right)\end{array}$ & $\begin{array}{c}\text { Heat Transfer } \\
\text { Coefficient \% } \\
\text { Difference with 3D } \\
\text { Steady-State }\end{array}$ \\
\hline $1.00 \mathrm{E}-04$ & 0.679 & 26.74 & 60.30 & 28.99 \\
\hline $2.00 \mathrm{E}-04$ & 0.512 & -4.32 & 51.64 & 10.47 \\
\hline $4.00 \mathrm{E}-04$ & 0.556 & 3.86 & 48.30 & 3.33 \\
\hline $6.00 \mathrm{E}-04$ & 0.548 & 2.44 & 46.85 & 0.22 \\
\hline $8.00 \mathrm{E}-04$ & 0.557 & 4.06 & 45.61 & -2.44 \\
\hline $1.00 \mathrm{E}-03$ & 0.557 & 4.08 & 45.56 & -2.54 \\
\hline $1.20 \mathrm{E}-03$ & 0.556 & 3.92 & 45.55 & -2.56 \\
\hline $1.40 \mathrm{E}-03$ & 0.556 & 3.82 & 45.55 & -2.55 \\
\hline
\end{tabular}

To put the heat flux on the target in perspective, published heat transfer correlations were plotted along with the 3D, steady-state heat flux plot in Figure 58. Belov and Terpigor'ev [3] developed a correlation predicting the Nusselt number $(\mathrm{Nu})$ at the stagnation point on a blunt object interacting with a jet of fluid. At stagnation, the correlation simplifies to

$$
N u_{d}=0.763 * \operatorname{Pr}^{0.4} * \sqrt{R e_{d}}
$$

where Pr is the Prandtl number and the Nusselt and Reynolds numbers are calculated with characteristic length equal to the diameter of the jet nozzle. For this application, it seemed reasonable to make this length equal to the diameter of the front wall. This correlation was adapted to predict heat flux on the front target wall. Hadad and Jafarpur [5] semi-analytically determined the average Nusselt number as a function of the Reynolds and Prandtl numbers for several objects in external flow. Their cylinder correlation was given as

$$
N u_{d}=1.5828+0.5506 * \operatorname{Re}_{d}^{1 / 2} * \operatorname{Pr}^{1 / 3}
$$


Note that this correlation is valid in the range $1<\operatorname{Re}_{d}>100$, and the Reynolds number in the drift region is approximately 4,000. Nevertheless, the correlation was adjusted to predict the average heat flux over a cylinder with diameter equal to the target's outer diameter. Kang and Sparrow [10] experimentally determined a correlation for the Nusselt number as a function of Reynolds number for flow around a cylinder. They developed the correlation not as an overall average, but as a function of the distance from the front of the cylinder. The correlation is approximated as

$$
N u_{x}=0.0426 * R e_{x}^{0.767}
$$

where $\mathrm{x}$ is the axial distance from the front of the cylinder. This correlation was adapted to predict heat flux along a cylinder with characteristic length equal to the axial distance from the front of the cylinder. Sogin [26] measured the heat transfer from the rear of bluff bodies in external flow and developed a correlation to predict the Nusselt number as a function of the Reynolds number:

$$
N u_{L}=0.20 * R e_{L}^{2 / 3}
$$

where $\mathrm{L}$ is the chord length of the flat plate strip. This correlation was adapted to predict the heat flux on the rear wall of an object with a flat plate chord length equal to the diameter of the target's rear wall. Note that the correlation is valid in the range of $10,000<\operatorname{Re}_{\mathrm{L}}<40,000$. The Reynolds number with characteristic length equal to the target's rear wall diameter is 2,620 . The heat flux plot from the $3 \mathrm{D}$, steady-state simulation can be seen in Figure 58 along with these 4 correlations. Note that the higherthan-average heat flux on the front and back walls aligns with the predictions by Belov and Terpigorev and Sogin. 


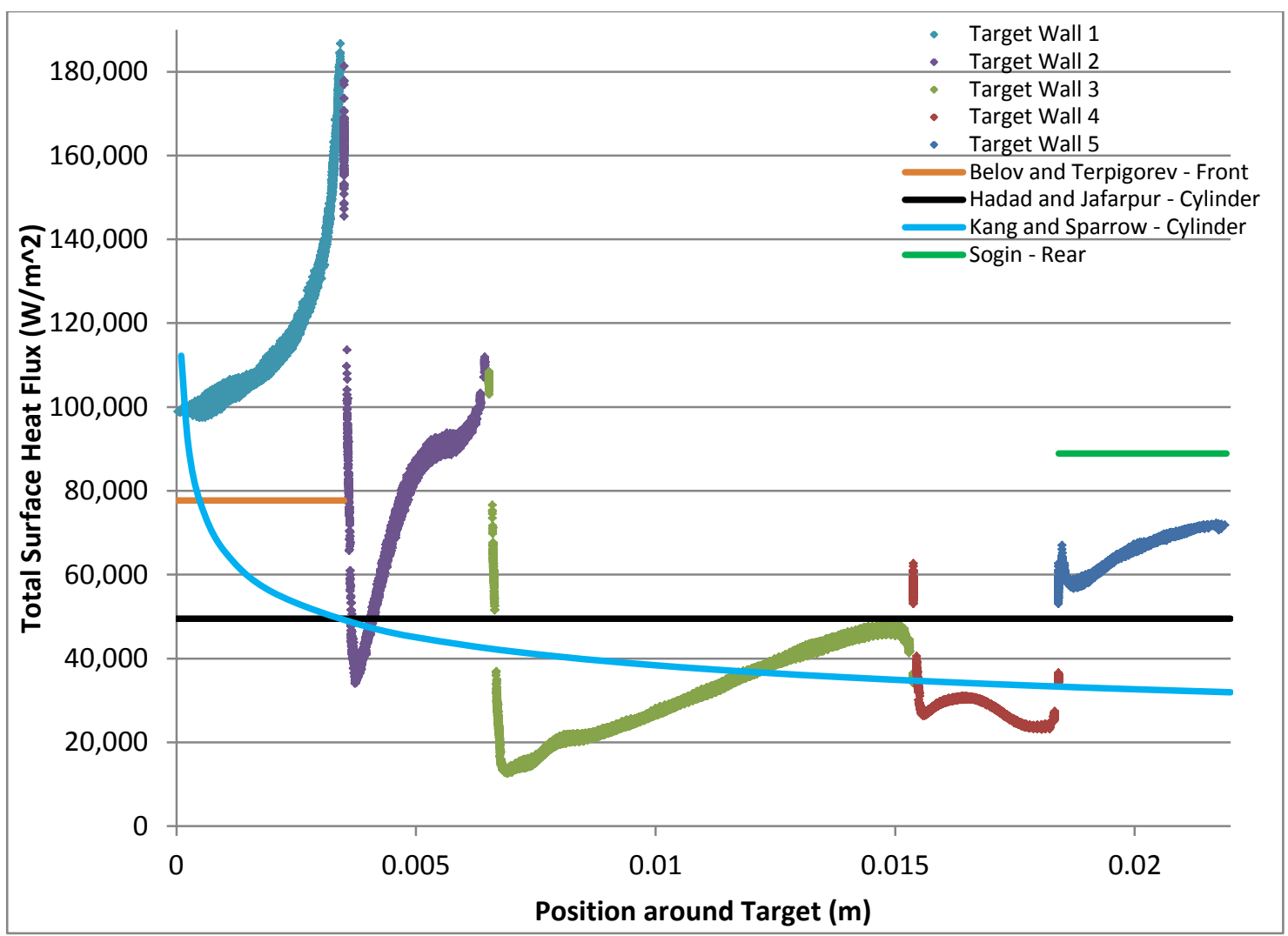

Figure 58. Heat Flux from 3D, Steady-State Case Plotted with Published Heat Transfer Correlations

Furthermore, the average heat coefficient can be calculated from the Hadad and Jafarpur [5] correlation and compared to the total heat coefficient from the 3D, steadystate simulation of $46.75 \mathrm{~W} /\left(\mathrm{m}^{2}-\mathrm{K}\right.$ ) (found in Table 17). The Nusselt correlation from Hadad and Jafarpur [5] predicts an average heat transfer coefficient of $48.11 \mathrm{~W} /\left(\mathrm{m}^{2}-\mathrm{K}\right)$, which is only $5.59 \%$ different than the total $3 \mathrm{D}$, steady-state simulation coefficient. Considering the obvious difference in shape and that all properties used to evaluate the Reynolds and Nusselt numbers for the published correlations were constants at the freestream temperature, these published correlations align closely to the heat transfer results from the 3D, steady-state simulation. 
Similarly, the value of the drag coefficient on the target from the 3D, steady-state simulations can be compared to the drag coefficient on a sphere predicted by Clift et al. [4]. Recall the Clift et al. correlation is valid for incompressible flow at a constant temperature. In the Reynolds range of 1,500 to 12,000, the correlation was given as [4]

$$
\log _{10} C_{D}=-2.4571+2.5558 * \log _{10} R e_{d}-0.9295 *\left(\log _{10} R e_{d}\right)^{2}+0.1049 *\left(\log _{10} R e_{d}\right)^{3}
$$

At a Reynolds number of 3,937 (the Reynolds number in the drift region), the predicted sphere drag coefficient is 0.3908 , which is $27.0 \%$ different from the 0.5354 coefficient from the 3D, steady-state simulation. Hadad and Jafarpur [5] determined a Nusselt correlation for flow over a sphere valid over a wide Reynolds number range of $1<\operatorname{Re}_{\mathrm{d}}<100,000$ :

$$
N u_{d}=2+0.5918 * R e_{d}^{1 / 2} * \operatorname{Pr}^{1 / 3}
$$

which would give the heat transfer coefficient in the drift region as $52.16 \mathrm{~W} /\left(\mathrm{m}^{2}-\mathrm{K}\right)$. Compared to the results from the 3D, steady-state simulation, this sphere heat transfer prediction has a $14.5 \%$ difference. For the same geometry, the predicted heat transfer coefficient has half the percent difference as the predicted drag coefficient. This may be attributed to the invalidity of the zero heat flux assumption (and possibly the incompressibility assumption) of the drag correlation as well as indicate the heat transfer correlations may predict values more accurately.

The total drag force and mass can be used to calculate acceleration (a) using Newton's second law [18],

$$
D=m a
$$

The acceleration due to the drag force from the 3D, steady-state simulation is approximately $47.9 \mathrm{~m} / \mathrm{s}^{2}$ opposing the direction of the flow. Using the velocityacceleration equation [18], 


$$
V=\sqrt{V_{0}^{2}+2 a s}
$$

(where the subscript 0 indicates the initial state and $\mathrm{s}$ is displacement in meters) the velocity after traveling $4 \mathrm{~m}$ through the drift region would be approximately $250.8 \mathrm{~m} / \mathrm{s}$, resulting in only a $0.32 \%$ change in velocity. Therefore the initial assumption of a constant-velocity target is reasonable.

As described in the CFD chapter, the drag and heat transfer on the 3D target were analyzed at different target velocities. Table 19 summarizes the results of the simulations, and Figures 59 and 60 plot the dimensionless drag coefficient and Nusselt number versus the Reynolds number.

Table 19. Drag and Heat Transfer from 3D, Steady-State Target Simulations at Various Reynolds Numbers

\begin{tabular}{|c|c|c|c|c|c|c|c|c|c|}
\hline Re & 2400 & 2800 & 3200 & 3600 & 3937 & 4400 & 4800 & 5200 & 5600 \\
\hline Drag Force (N) & 0.0416 & 0.0571 & 0.0859 & 0.1052 & 0.1452 & 0.2290 & 0.3020 & 0.4774 & 0.5859 \\
\hline Drag Coefficient & 0.4106 & 0.4145 & 0.4379 & 0.4618 & 0.5354 & 0.6729 & 0.7456 & 1.0042 & 1.0628 \\
\hline Heat Transfer (W) & 20.290 & 21.933 & 24.349 & 25.149 & 25.788 & 28.576 & 30.981 & 32.183 & 35.803 \\
\hline Nusselt Number & 24.288 & 26.255 & 29.148 & 30.106 & 30.871 & 34.208 & 37.087 & 38.526 & 42.860 \\
\hline
\end{tabular}

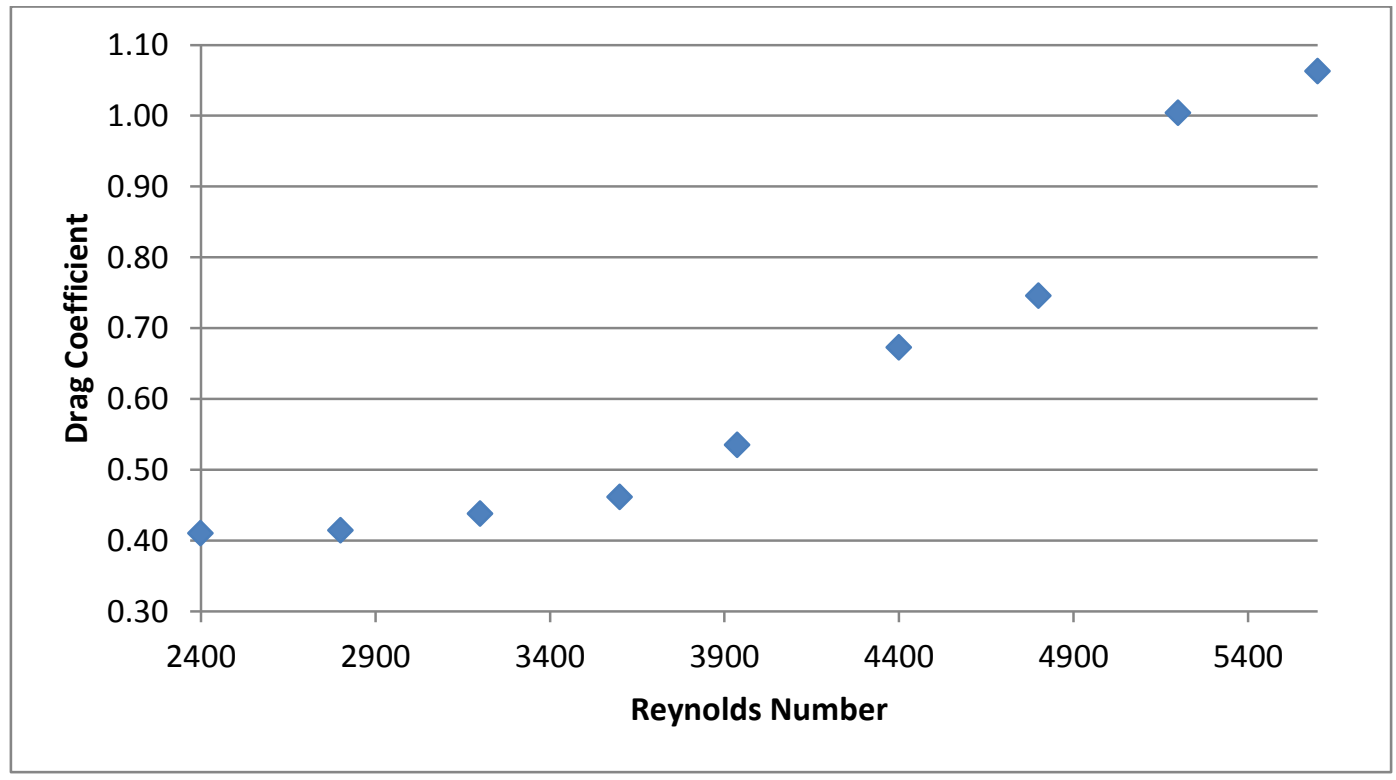

Figure 59. Drag Coefficient Plotted for 3D, Steady-State Target Simulations at Various Reynolds Numbers 


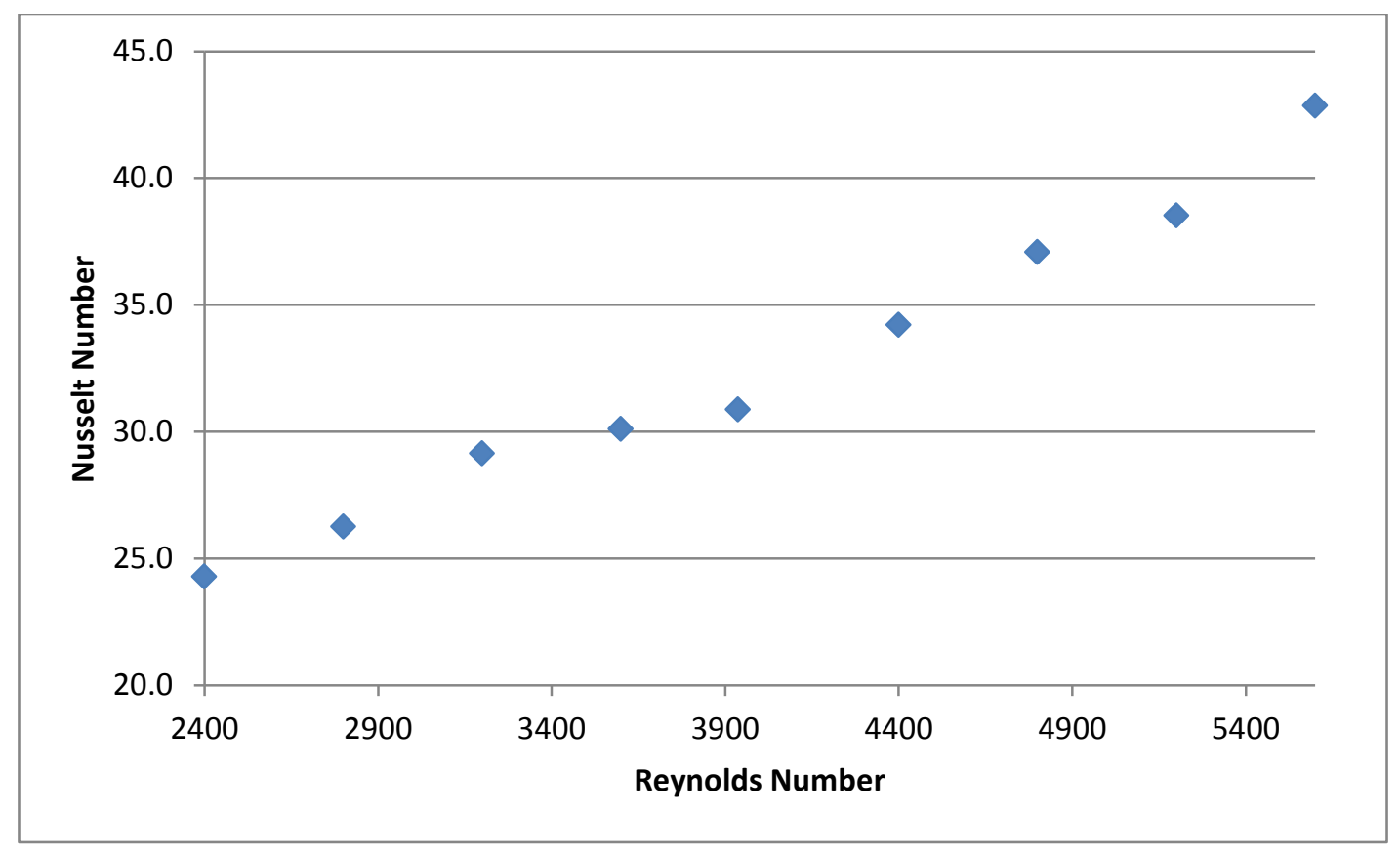

Figure 60. Nusselt Number Plotted for 3D, Steady-State Target Simulations at Various Reynolds Numbers

Excel was used to determine best-fit correlations for the drag coefficient and Nusselt number as functions of the Reynolds number in the Reynolds number range $2,400-5,600$. The best-fit, $2^{\text {nd }}$ order polynomial of the drag coefficient is

$$
C_{D}=(7.7468 E-8)\left(R e_{d}^{2}\right)-(4.0284 E-4) R e_{d}+0.92913
$$

And the best-fit power law for the Nusselt number is

$$
N u_{d}=0.1681 * R e_{d}^{0.6361}
$$

The $\mathrm{R}^{2}$ value for each is 0.9818 and 0.9733 , respectively, indicating a reasonable curve fit. Note that the published Nusselt-Reynolds correlations used as points of comparison for the target heat transfer have power-law exponents ranging from 0.5 to 0.767 . The best-fit Nusselt number correlation for the target has a power-law exponent of 0.6361 , which is within this range and, therefore, sensible. Pressure, temperature, and velocity contours with streamlines were plotted for each Reynolds number case. They can be seen in Figures 83-106 in Appendix C. 
Note the residuals in the simulation at a Reynolds number of 2,400 (velocity of $152.74 \mathrm{~m} / \mathrm{s}$ ) leveled off before dropping 4 orders of magnitude. All cases done with varying Reynolds number used the SST k-omega turbulence model. However, at this low Reynolds number, this model may not be as accurate as a laminar model. The residuals from this simulation only dropped 3 orders of magnitude. However, a popular criterion to determine convergence is an absolute convergence of $10^{-3}[1]$. Therefore, the results have been included and presented in the section. All residuals from the other simulations in this section dropped over 4 orders of magnitude.

As discussed in the CFD chapter, the target's angle of attack was altered to provide insight into the target's flight stability. Velocity contours with streamlines are shown at different angles of attack in Figures 61-66. Notice as the angle of attack increases, the symmetry of the flow around the target decreases and eventually one asymmetric wake region forms. The pressure and temperature contours for each angle of attack simulation can be found in Figures 107-118 in Appendix C.

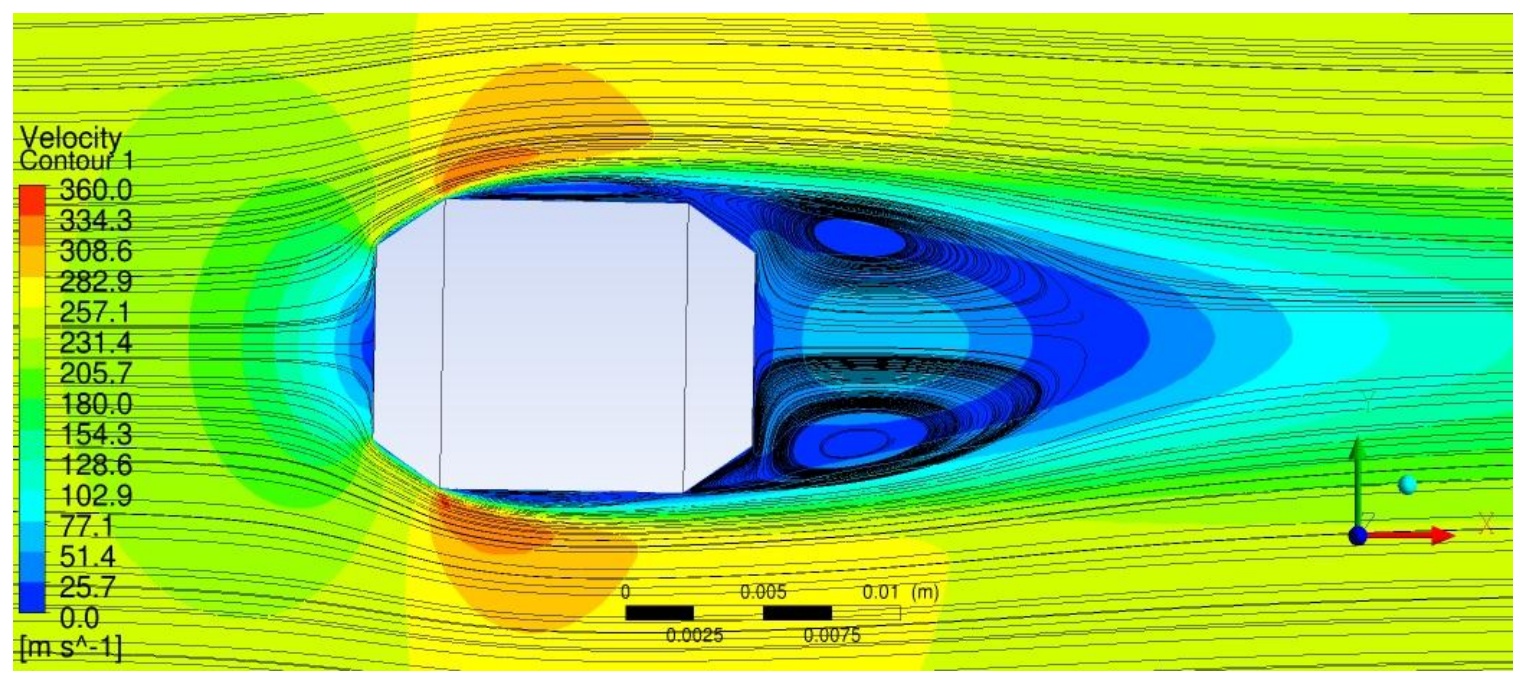

Figure 61. Velocity Contour and Streamline Plot for Target in 3D, Steady-State, Drift Simulation with $1^{\circ}$ Angle of Attack 


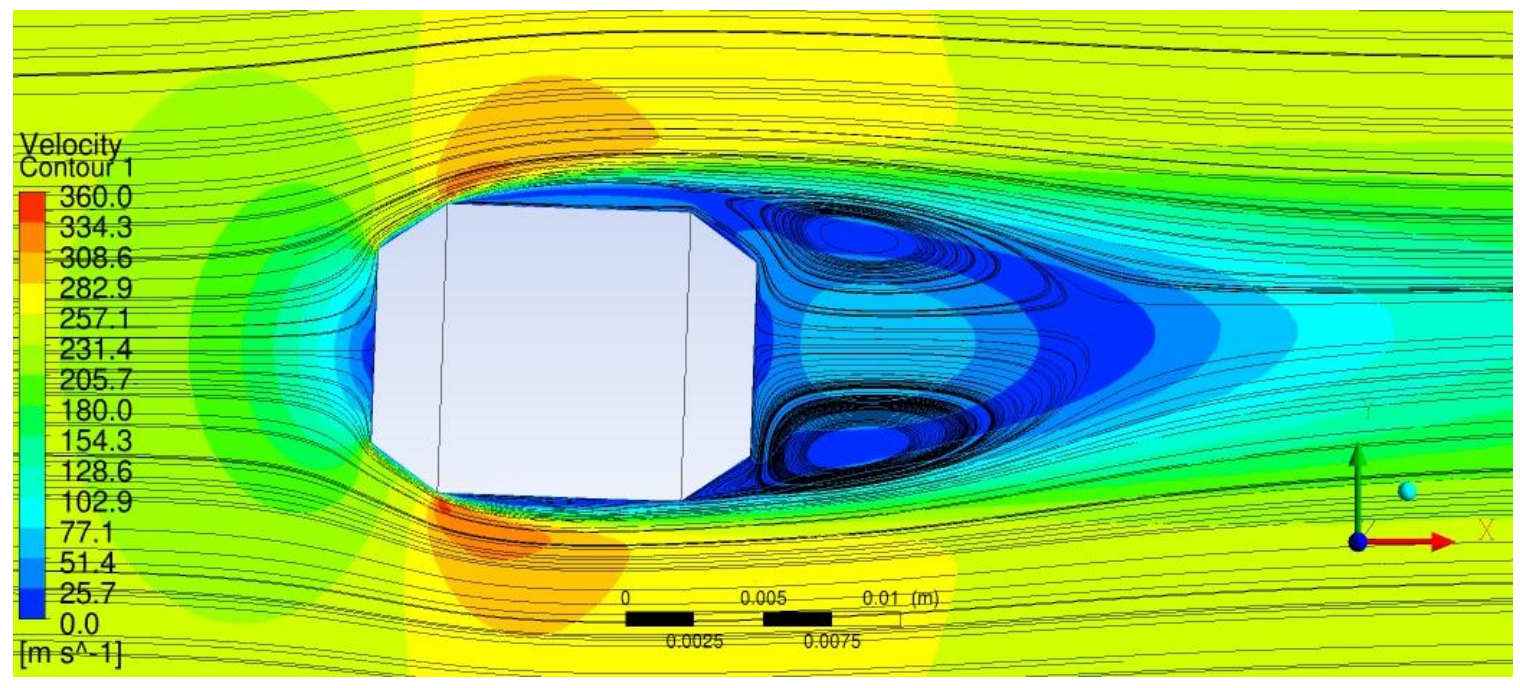

Figure 62. Velocity Contour and Streamline Plot for Target in 3D, Steady-State, Drift Simulation with $2^{\circ}$ Angle of Attack

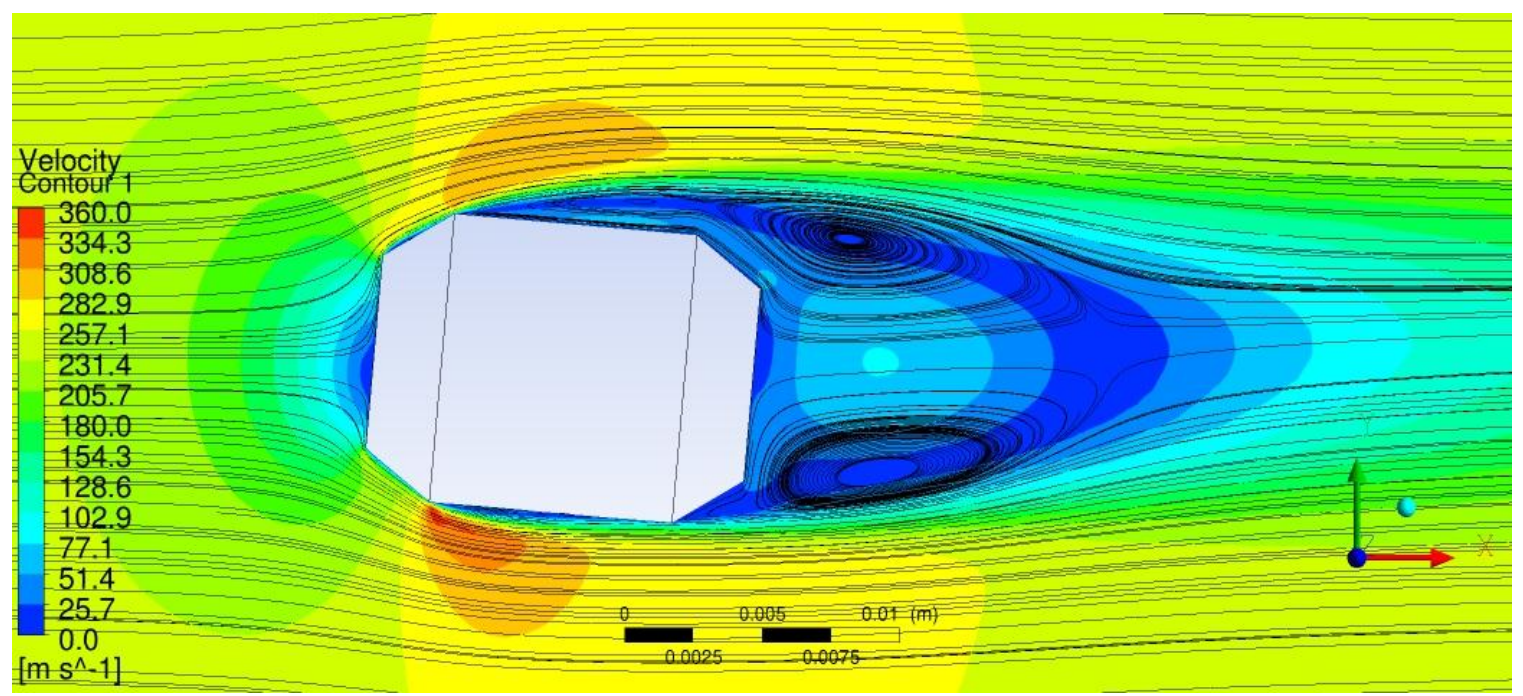

Figure 63. Velocity Contour and Streamline Plot for Target in 3D, Steady-State, Drift Simulation with $5^{\circ}$ Angle of Attack 


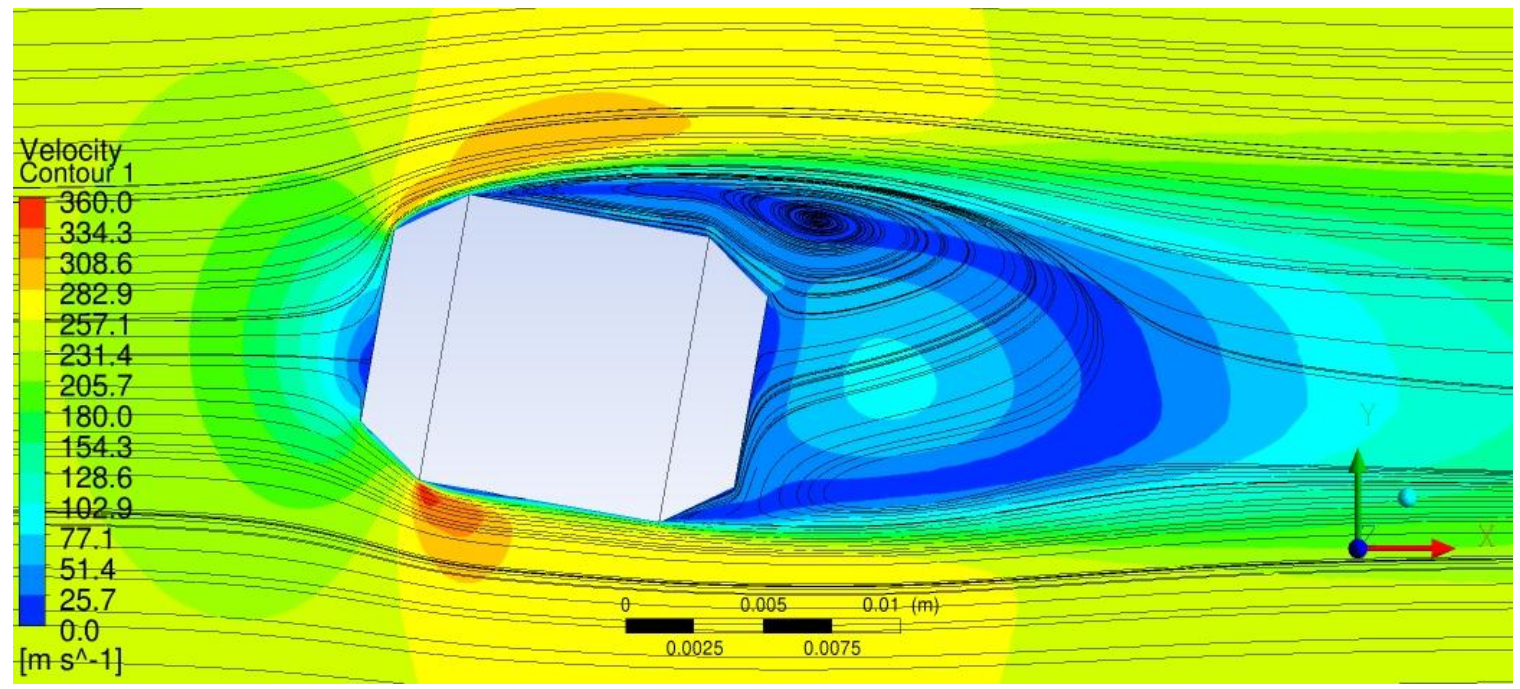

Figure 64. Velocity Contour and Streamline Plot for Target in 3D, Steady-State, Drift Simulation with $10^{\circ}$ Angle of Attack

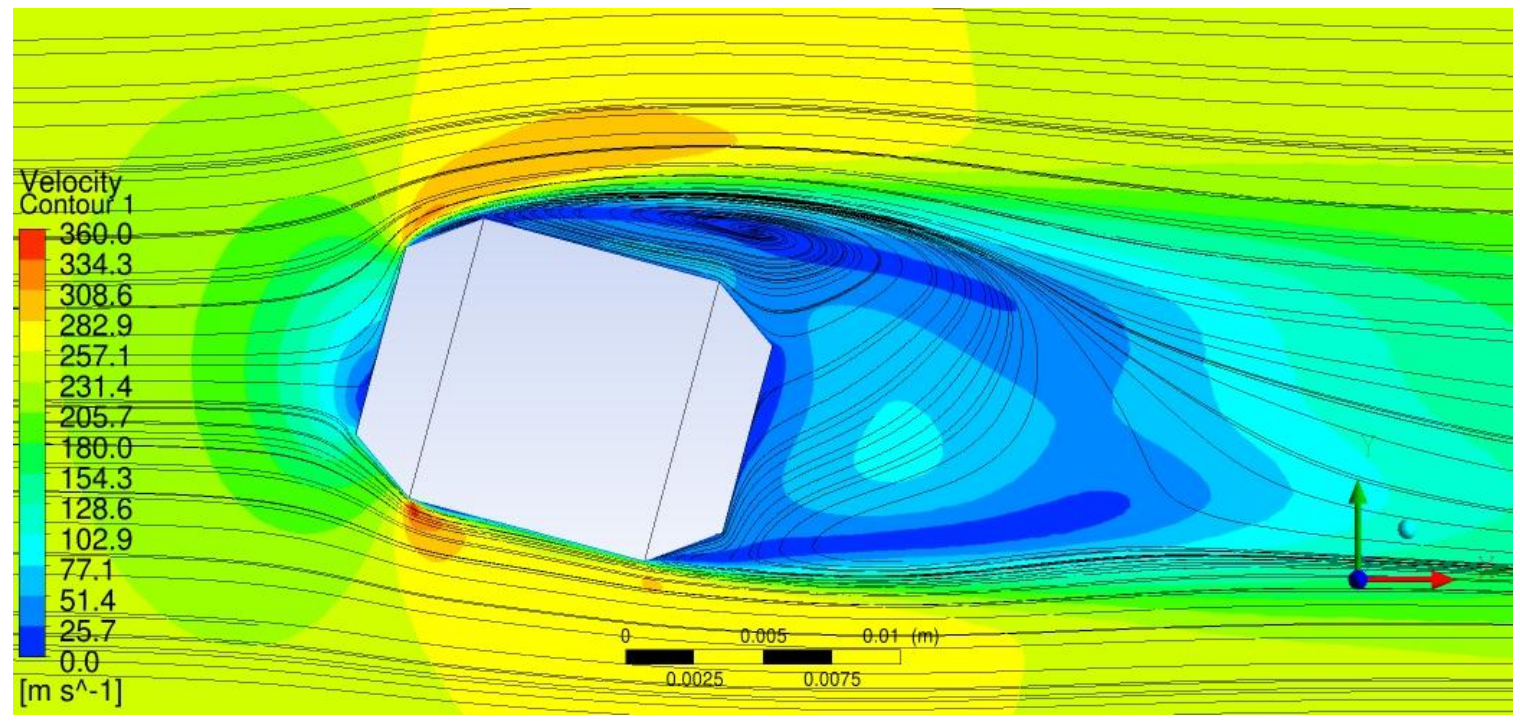

Figure 65. Velocity Contour and Streamline Plot for Target in 3D, Steady-State, Drift Simulation with $15^{\circ}$ Angle of Attack 


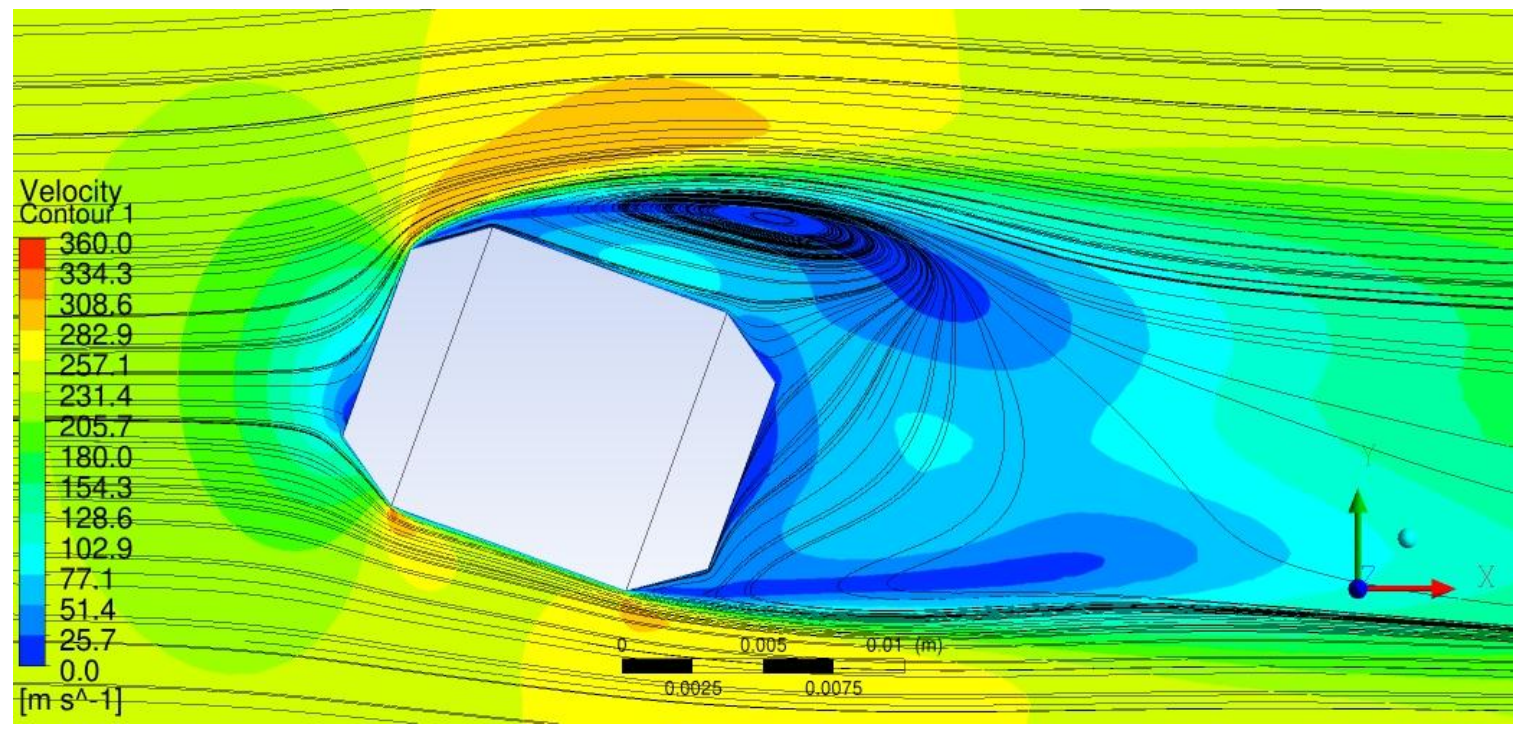

Figure 66. Velocity Contour and Streamline Plot for Target in 3D, Steady-State, Drift Simulation with $20^{\circ}$ Angle of Attack

The forces and moments on the target at the different angles of attack were plotted in Figures 67 and 68. Simulations were done with the target spinning and not spinning. Individual force and moment plots can be seen in Figures 119-130 in Appendix C. Note that the drag force, lift force, and z-axis moment changed minimally with spin. This is to be expected, as "static aerodynamics [such as the drag force, lift force, and z-axis moment] for spinning axisymmetric bodies are generally invariant with spin rate" [29]. Since the forces act at the center of pressure [17], local x-axis and local y-axis forces act through the local $\mathrm{y}$-axis and local $\mathrm{x}$-axis moment arms (distance from the center of gravity to the center of pressure in the local $y$-axis and local $\mathrm{x}$-axis directions) to create the z-axis moment. Therefore, it is logical that minimal change due to spin in the drag and lift forces (which are simple transformations of the local $\mathrm{x}$-axis and local $\mathrm{y}$-axis forces) would cause minimal change in the z-axis moment. 


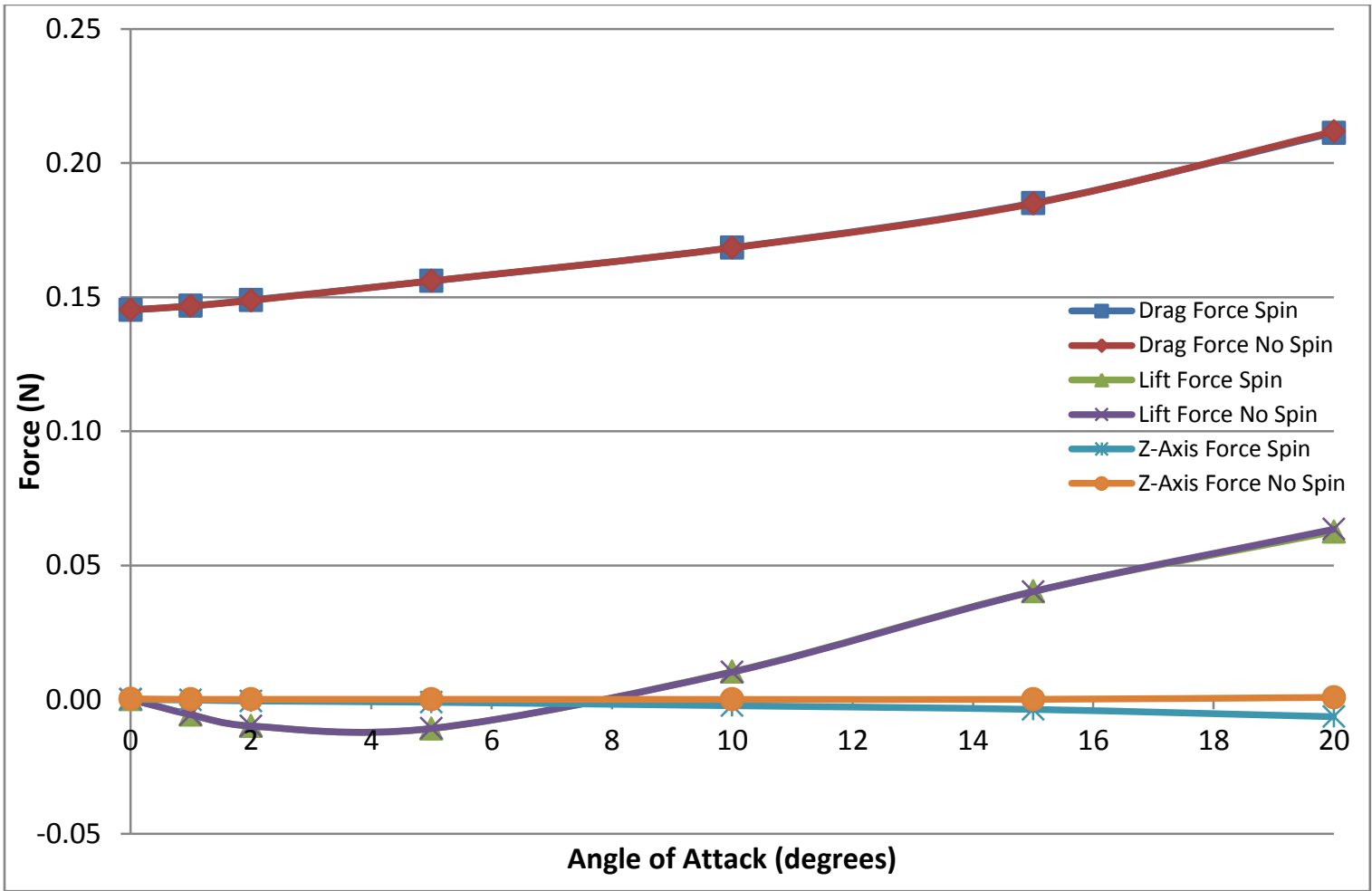

Figure 67. Forces on Spinning and Non-Spinning Target in Drift Region at Different Angles of Attack from 3D, Steady-State Model

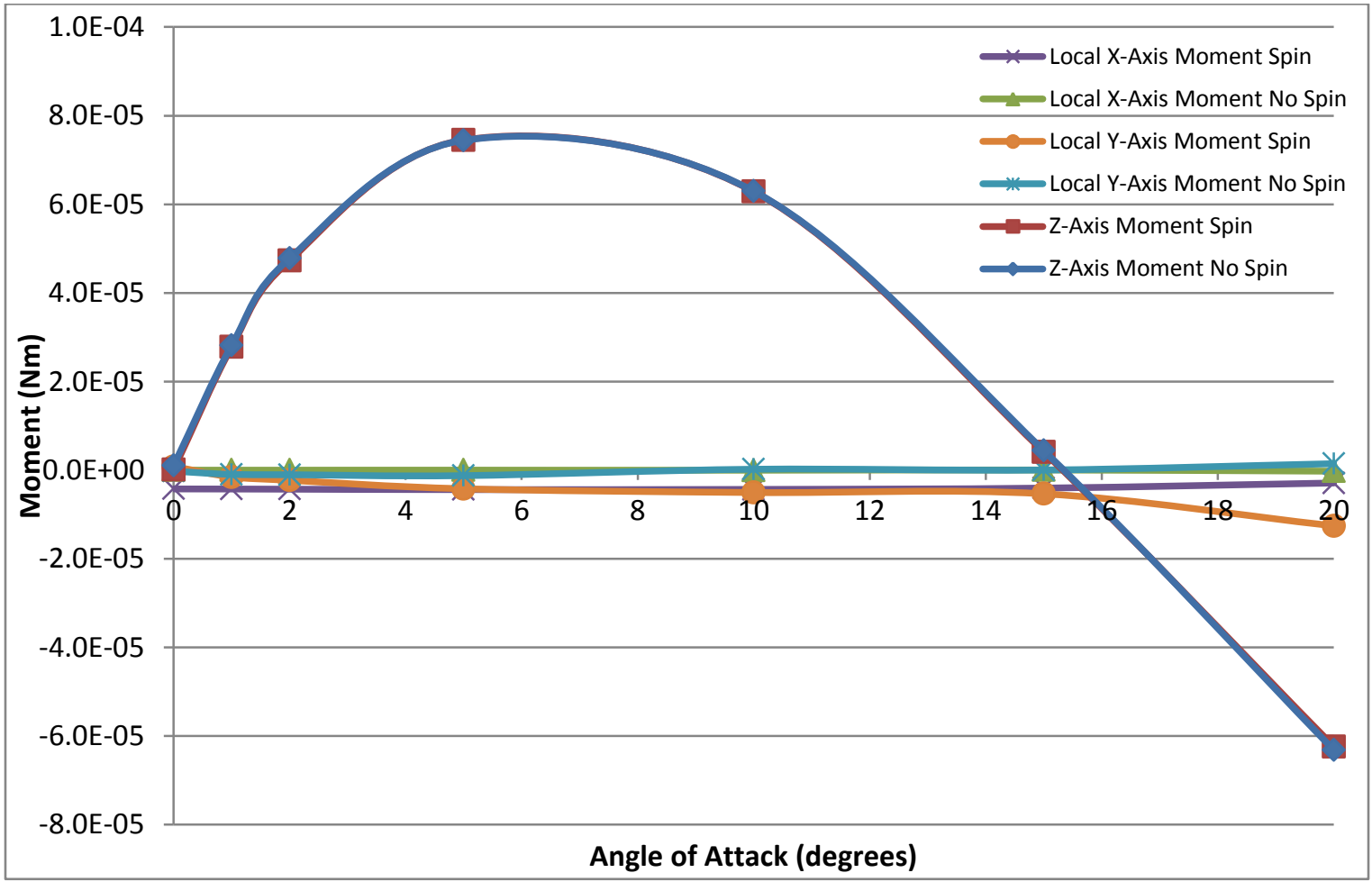

Figure 68. Moments on Spinning and Non-Spinning Target in Drift Region at Different Angles of Attack from 3D, Steady-State Model 
In Figure 67, the drag force increases as the angle of attack increases. Since the frontal projected area increases as the angle of attack increases, an increase in drag seems physically reasonable. Also note that the lift force reaches a minimum point at approximately $4^{\circ}$ and then appears to increase as the angle of attack increases. From the velocity contour and streamline plots, it appears that only walls 1 and 2 interact with the frontal incoming jet at small angles of attack. As the angle of attack increases, wall 3 eventually joins walls 1 and 2 in interacting with the incoming fluid. This frontal stream that interacts with wall 3 is forced downward around the target. This causes an equal and opposite force (upward lift) on the target by Newton's third law [18].

As can be seen in the velocity contour and streamline plots, the angle of attack simulations were created by rotating the target about the negative z-axis. From Figure 68 , the z-axis moment above approximately $15^{\circ}$ is negative and would increase the angle of attack, indicating a destabilizing moment. However, at small angles of attack, the zaxis moment is positive, which would tend to decrease the angle of attack and tend to stabilize the target. Note that the negative z-axis moment is the overturning moment.

The static aerodynamic forces and moments (drag force, lift force, and overturning moment) were determined from the non-spinning target simulations, and the Magnus moment (a dynamic moment) was determined from the spinning target simulations. This approach was recommended as aeroballistic standard practice [29]. Using these forces and moments and the McCoy [17] equations (reproduced in the background chapter), the aerodynamic coefficients of drag, lift, Magnus moment, and overturning moment were calculated and used to determine the target's static stability at different angles of attack. See Table 20. Note that the target is statically stable at or 
below an angle of attack of $15^{\circ}$, indicating the target does not need spin to be stabilized at these low angles of attack. However, if the target's angle of attack is greater than $15^{\circ}$, spin would be required to dynamically stabilize the target.

Table 20. Aerodynamic Coefficients and Static Stability of Target in Drift Region at Different Angles of Attack

\begin{tabular}{|c|c|c|c|c|c|c|}
\hline Angle of Attack & $1^{\circ}$ & $2^{\circ}$ & $5^{\circ}$ & $10^{\circ}$ & $15^{\circ}$ & $20^{\circ}$ \\
\hline Drag Coefficient, $\mathbf{C}_{\mathbf{D}}$ & 0.5406 & 0.5484 & 0.5752 & 0.6207 & 0.6815 & 0.7805 \\
\hline Lift Coefficient, $\mathbf{C}_{\mathbf{L}}$ & -1.1948 & -1.0476 & -0.4557 & 0.2170 & 0.5726 & 0.6841 \\
\hline $\begin{array}{c}\text { Magnus Moment } \\
\text { Coefficient, } C_{M_{p \alpha}}\end{array}$ & $5.200 \mathrm{E}-05$ & $3.851 \mathrm{E}-05$ & $2.832 \mathrm{E}-05$ & $1.708 \mathrm{E}-05$ & $1.211 \mathrm{E}-05$ & $2.156 \mathrm{E}-05$ \\
\hline $\begin{array}{c}\text { Overturning Moment } \\
\text { Coefficient, } C_{M_{\alpha}}\end{array}$ & -0.56632 & -0.48095 & -0.29985 & -0.12727 & -0.00599 & 0.06483 \\
\hline $\begin{array}{c}\text { Starred Overturning } \\
\text { Moment Coefficient, } C_{M_{\alpha}}\end{array}$ & $-8.509 \mathrm{E}-06$ & $-7.226 \mathrm{E}-06$ & $-4.505 \mathrm{E}-06$ & $-1.912 \mathrm{E}-06$ & $-9.005 \mathrm{E}-08$ & $9.741 \mathrm{E}-07$ \\
\hline $\mathbf{M}$ & $-3.309 \mathrm{E}-17$ & $-2.811 \mathrm{E}-17$ & $-1.752 \mathrm{E}-17$ & $-7.437 \mathrm{E}-18$ & $-3.502 \mathrm{E}-19$ & $3.789 \mathrm{E}-18$ \\
\hline Statically Stable? & Yes & Yes & Yes & Yes & Yes & No \\
\hline
\end{tabular}

If injected with an angle of attack less than $15^{\circ}$, the target would oscillate between positive and negative angles of attack (of decreasing magnitude) about the axis parallel to the free-stream flow. Since the overturning moment is typically calculated from nonspinning simulations, Excel was used to determine the best-fit polynomial for the z-axis moment on the non-spinning target as a function of the angle of attack:

$$
M_{z}=(5.265 E-8) A^{3}-(2.510 E-6) A^{2}+(2.584 E-5) A+3.290 E-6
$$

where $\mathrm{M}_{\mathrm{z}}$ is the moment about the $\mathrm{z}$-axis (the negative of the overturning moment) in $\mathrm{Nm}$ and $\mathrm{A}$ is the angle of attack in degrees. Using rotational kinematic equations of motion from Meriam [18] and the best-fit polynomial equation for the z-axis moment, the angle of attack was plotted over time with MATLAB with different initial angles of attack. Note that this motion is for a non-spinning target. However, since the duration of the flight through the drift region is only approximately $0.16 \mathrm{~ms}$ and the aspect ratio of the 
target is short, dynamic effects such as pitch damping would be minimal. Therefore, the oscillatory motion can be viewed as an approximation for a spinning target about a coordinate system that rotates at the target spin rate. See Appendix D for the MATLAB code including the kinematic equations used. Figures 131-135 in Appendix D show the plots of the angle of attack versus time for different initial angles of attack. Table 21 includes the period of oscillation for each initial angle of attack case. Since the flight in the drift region is only approximately $0.016 \mathrm{~s}$, the target wouldn't complete an oscillation starting with any of the initial angle of attacks modeled. Also, the change in amplitude of the oscillations indicates the effect of damping due to the overturning moment during this short flight is negligible. The exiting angle of attack after $0.016 \mathrm{~s}$ for each initial case is also included in this table.

Table 21. Angle of Attack Oscillation and Exit Value Determined by MATLAB Code

\begin{tabular}{|c|c|c|c|c|c|}
\hline Angle of Attack & $1^{\circ}$ & $2^{\circ}$ & $5^{\circ}$ & $10^{\circ}$ & $15^{\circ}$ \\
\hline Period of Oscillation & $0.0461 \mathrm{~s}$ & $0.0499 \mathrm{~s}$ & $0.0574 \mathrm{~s}$ & $0.0832 \mathrm{~s}$ & $0.2061 \mathrm{~s}$ \\
\hline Peak Amplitude After 25 Periods & $0.9994^{\circ}$ & $1.999^{\circ}$ & $4.998^{\circ}$ & $9.995^{\circ}$ & $14.94^{\circ}$ \\
\hline Change in Amplitude After 25 Periods & $0.0006^{\circ}$ & $0.001^{\circ}$ & 0.002 & $0.005^{\circ}$ & $0.06^{\circ}$ \\
\hline Angle of Attack after 0.016 s & $-0.585^{\circ}$ & $-0.882^{\circ}$ & $-0.684^{\circ}$ & $4.115^{\circ}$ & $14.600^{\circ}$ \\
\hline Change in Angle of Attack & $1.585^{\circ}$ & $2.882^{\circ}$ & $5.684^{\circ}$ & $5.885^{\circ}$ & $0.400^{\circ}$ \\
\hline
\end{tabular}

Further investigation of target flight stability could include simulating steady coning motion to determine the pitch damping moment at different angles of attack. The pitch damping moment coefficient can be used with the other aerodynamic coefficients presented in Table 20 to determine the dynamic stability factor. This would allow the calculation of a spin rate limit for the statically stable (low angle of attack) flight as well as determine if the target can be spin-stabilized above an angle of attack of $15^{\circ}$ and the spin rate required to do so. 


\section{CONCLUSIONS}

The 2D and 3D simulations of flow over the LIFE target indicate that the flow is axisymmetric and steady for the majority of the flight. The drag and heat transfer on each target face was provided along with correlations for the drag coefficient and Nusselt number as functions of the Reynolds number.

Experimental results of flow over a LIFE target in the drift region would be the ideal source of comparison for the target simulations. However, the flow over the target experiences many of the same trends in velocity, pressure, steady wake structure, and wake symmetry as low Reynolds number flow over a sphere. The high levels of accuracy from the sphere simulations with the same mesh generation method and at the same drift conditions serve as validation for the simulations of the flow over the target.

Furthermore, the target simulation's heat transfer predictions aligned well with those from an experimentally and analytically determined Nusselt correlation for a cylinder.

If injected at low angles of attack (less than $15^{\circ}$ ), the target would be statically stable, implying target spin would not be required for stability. At these low initial angles of attack, the target would oscillate between positive and negative angles of attack (of decreasing magnitude) about the axis parallel to the free-stream flow. The exiting angle of attack at the end of the drift region and the period of oscillation for different initial angles of attack were tabulated. These results can have significant implications on the requirements and design of the target injection system.

Future work could consist of determining the pitch damping moment through steady coning motion simulations. The pitch damping moment coefficient along with the other aerodynamic coefficients presented in the results section could be used to solve the simultaneous differential equations of motion presented in McCoy's 6-degrees-of- 
freedom section [17]. A 6-degree-of-freedom Fluent simulation could be created and run, and the results could be compared to the predictions given by McCoy's equations of motion. Additionally, the transition from the injector to the drift region and from the drift region to the chamber could be explored with transient simulations. 


\section{BIBLIOGRAPHY}

[1] ANSYS, Inc. (2012). ANSYS Fluent user's guide, 14.5. Pennsylvania.

[2] Bailey, A. B., \& Hiatt, J. (1972). Sphere drag coefficients for a broad range of mach and Reynolds numbers. AIAA Journal, 10, 1436-1440.

[3] Belov, A., \& Terpigor'ev, V. S. (1972). Turbulence in calculations of heat transfer at the stagnation point of a jet interacting normally with a planar obstacle. New York: Plenum Publishing Corporation.

[4] Clift, R., Grace, J. R., \& Weber, M. E. (1978). Bubbles, drops, and particles. New York, NY: Academic Press, Inc.

[5] Hadad, Y., \& Jafarpur, K. (2008, August). Laminar forced convection heat transfer from isothermal bodies with unit aspect ratio. Paper presented at the $6^{\text {th }}$ IASME/WSEAS International Conference on Heat Transfer, Thermal Engineering, and Environment, Rhodes, Greece.

[6] Heller, A. (2011, July/August). Igniting our energy future. Science and Technology Review, 4-12.

[7] Hirschfelder, J. O., Curtiss, C. F., \& Bird, R. B. (1964). The molecular theory of gases and liquids. New York: John Wiley \& Sons, Inc.

[8] Incropera, F. P., Dewitt, D. P., Bergman, T. L., \& Lavine, A. S. (2007). Introduction to heat transfer $\left(5^{\text {th }}\right.$ ed. $)$. John Wiley \& Sons, Inc.

[9] Jones, D. A., \& Clarke, D. B. (2008). Simulation of flow past a sphere using the Fluent code. Victoria, Australia: Maritime Platforms Division.

[10] Kang, S. S., \& Sparrow, E. M. (1987). Heat transfer from an open- or closed- bore cylinder situated longitudinal to a freestream. Transactions of the ASME: Journal of Heat Transfer, 109, 314-320. 
[11] Kestin, J., Ro, S. T., \& Wakeham, W. (1972). An extended law of corresponding states for the equilibrium and transport properties of the noble gases. Physica, 58, $165-211$.

[12] Lawrence Livermore National Laboratory. How ICF works. NIF. Retrieved July 30, 2013.

[13] Lawrence Livermore National Laboratory. Inertial confinement fusion: How to make a star. NIF. Retrieved July 30, 2013.

[14] Lawrence Livermore National Laboratory. LIFE inside and out [Online image].

Retrieved December 9, 2013, from

https://lasers.llnl.gov/multimedia/photo_gallery/life/?id=1\&category=life

[15] Lawrence Livermore National Laboratory. NIF hohlraum [Online photograph].

Retrieved December 9, 2013, from

https://lasers.llnl.gov/multimedia/photo_gallery/target_area/?id=1\&category=targ et_area

[16] Lawrence Livermore National Laboratory. The power of light [Online simulation]. Retrieved December 9, 2013, from https://lasers.llnl.gov/multimedia/video_gallery/the_power_of_light.php

[17] McCoy, R. L. (1999). Modern exterior ballistics: The launch and flight dynamics of symmetric projectiles. Pennsylvania: Schiffer Publishing Ltd.

[18] Meriam, J. L., \& Kraige, L. G. (2007). Engineering mechanics: Dynamics $\left(6^{\text {th }}\right.$ ed.). John Wiley \& Sons, Inc.

[19] Moran, M. J., \& Shapiro, H. N. (2008). Fundamentals of engineering thermodynamics $\left(6^{\text {th }}\right.$ ed.). John Wiley \& Sons, Inc. 
[20] Munson, B. R., Young, D. R., Okiishi, T. H., \& Huebsch, W. W. (2009).

Fundamentals of fluid mechanics $\left(6^{\text {th }}\right.$ ed.). John Wiley \& Sons, Inc.

[21] Nakamura, I. (1976). Steady wake behind a sphere. The Physics of Fluids, 19, 5-8.

[22] National Institute of Standards and Technology. (2010). Fundamental physical constants. The NIST Reference on Constants, Units, and Uncertainty. Retrieved July 15, 2013.

[23] Reid, R. C., Prausnitz, \& Poling, J. M., B. E. (1987). The properties of gases \& liquids $\left(4^{\text {th }}\right.$ ed.). McGraw-Hill.

[24] Roos, F. W., \& Willmarth, W. W. (1971). Some experimental results on sphere and disk drag. AIAA Journal, 9, 285-291.

[25] Sakamoto, H., \& Haniu, H. (1990). A study on vortex shedding from spheres in a uniform flow. Transactions of the ASME: Journal of Fluids Engineering, 112, 386-392.

[26] Sogin, H. H. (1964). A summary of experiments on local heat transfer from the rear of bluff obstacles to a low speed airstream. Transactions of the ASME: Journal of Heat Transfer, 86, 200-202.

[27] Taneda, S. (1956). Experimental investigation of the wake behind a sphere at low Reynolds numbers. Journal of the Physical Society of Japan, 11, 1104-1108.

[28] Thomas Jefferson National Accelerator Facility - Office of Science Education. The element xenon. It's Elemental. Retrieved July 16, 2013.

[29] Weinacht, P. (2003). Prediction of projectile performance, stability, and free-flight motion using computational fluid dynamics. Maryland: Army Research Laboratory. 
[30] Wolfram | Alpha. (2013). Wolfram Alpha LLC. Retrieved July 16, 2013 from http://www.wolframalpha.com/input/?i=xenon+phase+diagram 


\section{APPENDICES}

\section{Appendix A: Fluent Setup}

Table 22. Detailed Fluent Case Setup for 2D, Axisymmetric Sphere in Drift Region

\begin{tabular}{|c|c|c|}
\hline \multicolumn{2}{|l|}{ Version } & 2D, double precision \\
\hline \multicolumn{2}{|c|}{ Space Model } & Axisymmetric \\
\hline \multicolumn{2}{|c|}{ Time Model } & Steady \\
\hline \multicolumn{2}{|c|}{ Velocity Formulation } & Absolute \\
\hline \multicolumn{2}{|c|}{ Viscous Model } & Laminar \\
\hline \multicolumn{2}{|c|}{ Heat Transfer Model } & Enabled \\
\hline \multicolumn{2}{|c|}{ Xenon Density } & Ideal gas \\
\hline \multicolumn{2}{|c|}{ Xenon Specific Heat $(\mathrm{J} /(\mathrm{kg}-\mathrm{K}))$} & 158.32 \\
\hline \multicolumn{2}{|c|}{ Xenon Thermal Conductivity $(\mathrm{W} /(\mathrm{m}-\mathrm{K}))$} & 0.0159 \\
\hline \multicolumn{2}{|c|}{ Xenon Viscosity $(\mathrm{kg} /(\mathrm{m}-\mathrm{s}))$} & $6.7 \mathrm{E}-5$ \\
\hline \multicolumn{2}{|c|}{ Xenon Molecular Weight (kg/kgmol) } & 131.293 \\
\hline \multicolumn{2}{|c|}{ Aluminum Density $\left(\mathrm{kg} / \mathrm{m}^{3}\right)$} & 2719 \\
\hline \multicolumn{2}{|c|}{ Aluminum Specific Heat $(\mathrm{J} /(\mathrm{kg}-\mathrm{K}))$} & 871 \\
\hline \multicolumn{2}{|c|}{ Aluminum Thermal Conductivity $(\mathrm{W} /(\mathrm{m}-\mathrm{K}))$} & 202.4 \\
\hline \multirow[t]{8}{*}{ Inlet } & Mass Flow Inlet & \\
\hline & Reference Frame & Absolute \\
\hline & Mass Flow Specification Method & Mass Flow Rate \\
\hline & Mass Flow Rate & Case dependent - see Table 4 \\
\hline & Supersonic/Initial Gauge Pressure & $0 \mathrm{~Pa}$ \\
\hline & Axial-component of flow direction & 1 \\
\hline & Radial-component of flow direction & 0 \\
\hline & Total temperature & Case dependent - see Table 4 \\
\hline Sphere & Stationary wall & \\
\hline & No slip & \\
\hline & Heat Flux & $0 \mathrm{~W} / \mathrm{m}^{2}$ \\
\hline & Heat generation rate & $0 \mathrm{~W} / \mathrm{m}^{3}$ \\
\hline & Material & Aluminum \\
\hline Outer Wall & Stationary wall & \\
\hline & Specified shear x-component & $0 \mathrm{~Pa}$ \\
\hline & Specified shear y-component & $0 \mathrm{~Pa}$ \\
\hline & Heat flux & $0 \mathrm{~W} / \mathrm{m}^{2}$ \\
\hline & Heat generation rate & $0 \mathrm{~W} / \mathrm{m}^{3}$ \\
\hline & Material & Aluminum \\
\hline Outlet & Pressure outlet & \\
\hline & Gauge pressure & $0 \mathrm{~Pa}$ \\
\hline & $\begin{array}{l}\text { Backflow direction specification } \\
\text { method }\end{array}$ & Normal to boundary \\
\hline & Backflow total temperature & Case dependent - see Table 4 \\
\hline
\end{tabular}




\begin{tabular}{|c|c|c|}
\hline \multicolumn{3}{|l|}{ Axis } \\
\hline Operating pressure & \multicolumn{2}{|l|}{$6666.118 \mathrm{~Pa}$} \\
\hline Solver & \multicolumn{2}{|l|}{ Pressure-based, Coupled } \\
\hline \multirow[t]{5}{*}{ Discretization Scheme } & Gradient & Green-Gauss Node Based \\
\hline & Pressure & Second order \\
\hline & Density & Second order upwind \\
\hline & Momentum & Second order upwind \\
\hline & Energy & Second order upwind \\
\hline \multirow[t]{5}{*}{ Pseudo Transient } & \multicolumn{2}{|l|}{ Enabled } \\
\hline & Time Step Method & Automatic \\
\hline & Timescale Factor & 1 \\
\hline & Length Scale Method & Conservative \\
\hline & Verbosity & 0 \\
\hline \multicolumn{2}{|c|}{ Pressure Relaxation Factor } & 0.5 \\
\hline \multicolumn{2}{|c|}{ Momentum Relaxation Factor } & 0.5 \\
\hline \multicolumn{2}{|c|}{ Density Relaxation Factor } & 1 \\
\hline \multicolumn{2}{|c|}{ Body Forces Relaxation Factor } & 1 \\
\hline \multicolumn{2}{|c|}{ Energy Relaxation Factor } & 0.75 \\
\hline \multicolumn{2}{|l|}{ Solution Limits } & Default \\
\hline \multirow[t]{2}{*}{ Residual Monitor } & $\begin{array}{l}\text { Absolute convergence } \\
\text { criteria (all) }\end{array}$ & $1 e-9$ \\
\hline & Iterations to store & 1000 \\
\hline Solution Initialization & Standard, absolute & Compute from inlet \\
\hline \multirow[t]{4}{*}{ Initial Values } & Gauge Pressure & $0 \mathrm{~Pa}$ \\
\hline & Axial Velocity & Case dependent - see Table 4 \\
\hline & Radial Velocity & $0 \mathrm{~m} / \mathrm{s}$ \\
\hline & Temperature & $1050 \mathrm{~K}$ \\
\hline \multicolumn{2}{|l|}{ Number of iterations } & 500 \\
\hline
\end{tabular}

Table 23. Detailed Fluent Case Setup for 2D, Axisymmetric Swirl Target in Drift Region

\begin{tabular}{|l|l|}
\hline Version & 2D, double precision \\
\hline Space Model & Axisymmetric swirl \\
\hline Time Model & Unsteady, $1^{\text {st }}$ order implicit \\
\hline Velocity Formulation & Absolute \\
\hline Viscous Model & SST k-omega turbulence \\
\hline Heat Transfer Model & Enabled \\
\hline Xenon Density & Ideal gas \\
\hline Xenon Specific Heat $(\mathrm{J} /(\mathrm{kg}-\mathrm{K}))$ & 158.32 \\
\hline Xenon Thermal Conductivity $(\mathrm{W} /(\mathrm{m}-\mathrm{K}))$ & $-0.0002769+2.088 \mathrm{E}-5 \mathrm{~T}-5.323 \mathrm{E}-9 \mathrm{~T}^{2}$ \\
\hline Xenon Viscosity $(\mathrm{kg} /(\mathrm{m}-\mathrm{s}))$ & $-1.167 \mathrm{E}-6+8.796 \mathrm{E}-8 \mathrm{~T}-2.243 \mathrm{E}-11 \mathrm{~T}^{2}$ \\
\hline
\end{tabular}




\begin{tabular}{|c|c|c|c|c|c|}
\hline \multicolumn{3}{|c|}{ Xenon Molecular Weight (kg/kgmol) } & \multicolumn{3}{|c|}{131.293} \\
\hline \multicolumn{3}{|c|}{ Aluminum Density $\left(\mathrm{kg} / \mathrm{m}^{3}\right)$} & \multicolumn{3}{|c|}{2719} \\
\hline \multicolumn{3}{|c|}{ Aluminum Specific Heat $(\mathrm{J} /(\mathrm{kg}-\mathrm{K}))$} & \multicolumn{3}{|l|}{871} \\
\hline \multicolumn{3}{|c|}{ Aluminum Thermal Conductivity $(\mathrm{W} /(\mathrm{m}-\mathrm{K}))$} & \multicolumn{3}{|c|}{202.4} \\
\hline \multirow[t]{9}{*}{ Inlet } & \multicolumn{4}{|c|}{ Far-field pressure } & \\
\hline & \multicolumn{4}{|c|}{ Gauge pressure } & $0 \mathrm{~Pa}$ \\
\hline & \multicolumn{4}{|c|}{ Mach number } & 0.7509739 \\
\hline & \multicolumn{4}{|c|}{ Temperature } & $1050 \mathrm{~K}$ \\
\hline & \multicolumn{4}{|c|}{ Axial-component of flow direction } & 1 \\
\hline & \multicolumn{4}{|c|}{ Radial-component of flow direction } & 0 \\
\hline & \multicolumn{4}{|c|}{ Tangential-component of flow direction } & 0 \\
\hline & \multicolumn{4}{|c|}{ Turbulent intensity } & $1 \%$ \\
\hline & \multicolumn{4}{|c|}{ Turbulent viscosity ratio } & $1 \%$ \\
\hline \multirow[t]{7}{*}{ Target $1-5$} & \multicolumn{4}{|c|}{ Moving wall with absolute, rotational motion } & $1570.796 \mathrm{rad} / \mathrm{s}$ \\
\hline & \multicolumn{4}{|c|}{ No slip } & \\
\hline & \multicolumn{4}{|c|}{ Roughness height } & $0 \mathrm{~m}$ \\
\hline & \multicolumn{4}{|c|}{ Roughness constant } & 0.5 \\
\hline & \multicolumn{4}{|c|}{ Temperature } & $20 \mathrm{~K}$ \\
\hline & \multicolumn{4}{|c|}{ Heat generation rate } & $0 \mathrm{~W} / \mathrm{m}^{3}$ \\
\hline & Material & & & & Aluminum \\
\hline Outer Wall & Stationar & wall & & & \\
\hline & Specified & shear x-component & & & $0 \mathrm{~Pa}$ \\
\hline & Specified & shear y-component & & & $0 \mathrm{~Pa}$ \\
\hline & Specified & shear z-component & & & $0 \mathrm{~Pa}$ \\
\hline & Roughne & height & & & $0 \mathrm{~m}$ \\
\hline & Roughne & constant & & & 0.5 \\
\hline & Heat flux & & & & $0 \mathrm{~W} / \mathrm{m}^{2}$ \\
\hline & Heat gen & ration rate & & & $0 \mathrm{~W} / \mathrm{m}^{3}$ \\
\hline & Material & & & & Aluminum \\
\hline Outlet & Pressure & utlet & & & \\
\hline & Gauge pr & ssure & & & $0 \mathrm{~Pa}$ \\
\hline & Backflon & direction specificatio & lethod & & Normal to boundary \\
\hline & Backflon & turbulent intensity & & & $1 \%$ \\
\hline & Backflon & turbulent viscosity ra & & & 1 \\
\hline & Backflon & total temperature & & & $1247.39 \mathrm{~K}$ \\
\hline Axis & Axis & & & & \\
\hline Operating $\mathrm{p}$ & essure & $6666.118 \mathrm{~Pa}$ & & & \\
\hline Solver & & Density-based, Impl & & & \\
\hline Flux Type & & Roe-FDS & & & \\
\hline Discretizatic & Scheme & Gradient & & & en-Gauss Node Based \\
\hline & & Flow & & & t order upwind \\
\hline & & Turbulent Kinetic E & & & t order upwind \\
\hline & & Specified Dissipatio & & & t order upwind \\
\hline
\end{tabular}




\begin{tabular}{|c|c|c|c|}
\hline \multicolumn{2}{|c|}{ Turbulent Kinetic Energy Relaxation Factor } & \multicolumn{2}{|l|}{0.8} \\
\hline \multicolumn{2}{|c|}{ Specified Dissipation Rate Relaxation Factor } & \multicolumn{2}{|l|}{0.8} \\
\hline \multicolumn{2}{|c|}{ Turbulent Viscosity Relaxation Factor } & \multicolumn{2}{|l|}{1} \\
\hline \multicolumn{2}{|c|}{ Solid Relaxation Factor } & \multicolumn{2}{|l|}{1} \\
\hline \multicolumn{2}{|l|}{ Courant Number } & \\
\hline \multicolumn{2}{|l|}{ Solution Limits } & & Default \\
\hline \multirow[t]{2}{*}{ Residual Monitor } & \multicolumn{2}{|l|}{ Absolute convergence criteria (all) } & $1 \mathrm{e}-4$ \\
\hline & \multicolumn{2}{|l|}{ Iterations to store } & 10000 \\
\hline \multirow{8}{*}{$\begin{array}{l}\text { Solution Initialization } \\
\text { Initial Values }\end{array}$} & \multicolumn{2}{|l|}{ Standard, absolute } & Compute from inlet \\
\hline & \multicolumn{2}{|l|}{ Gauge Pressure } & $0 \mathrm{~Pa}$ \\
\hline & \multicolumn{2}{|l|}{ Axial Velocity } & $250 \mathrm{~m} / \mathrm{s}$ \\
\hline & \multicolumn{2}{|l|}{ Radial Velocity } & $0 \mathrm{~m} / \mathrm{s}$ \\
\hline & \multicolumn{2}{|l|}{ Swirl Velocity } & $0 \mathrm{~m} / \mathrm{s}$ \\
\hline & \multicolumn{2}{|l|}{ Turbulent Kinetic Energy } & $9.37473 \mathrm{~m}^{2} / \mathrm{s}^{2}$ \\
\hline & \multicolumn{2}{|l|}{ Specific Dissipation Rate } & $52213.611 / \mathrm{s}$ \\
\hline & \multicolumn{2}{|l|}{ Temperature } & $1050 \mathrm{~K}$ \\
\hline \multicolumn{3}{|l|}{ Time step } & $5 \mathrm{E}-7 \mathrm{~s}$ \\
\hline \multicolumn{3}{|c|}{ Number of time steps } & 2000 \\
\hline \multicolumn{3}{|c|}{ Max Iterations per time step } & 150 \\
\hline
\end{tabular}

Table 24. Detailed Fluent Case Setup for 3D, Steady-State Target in Drift Region

\begin{tabular}{|c|c|c|c|}
\hline \multicolumn{2}{|c|}{ Version } & \multicolumn{2}{|c|}{ 3D, double precision } \\
\hline \multicolumn{2}{|c|}{ Time Model } & \multicolumn{2}{|c|}{ Steady } \\
\hline \multicolumn{2}{|c|}{ Velocity Formulation } & \multicolumn{2}{|c|}{ Absolute } \\
\hline \multicolumn{2}{|c|}{ Viscous Model } & \multicolumn{2}{|c|}{ SST k-omega turbulence } \\
\hline \multicolumn{2}{|c|}{ Heat Transfer Model } & \multicolumn{2}{|c|}{ Enabled } \\
\hline \multicolumn{2}{|c|}{ Xenon Density } & \multicolumn{2}{|c|}{ Ideal gas } \\
\hline \multicolumn{2}{|c|}{ Xenon Specific Heat $(\mathrm{J} /(\mathrm{kg}-\mathrm{K}))$} & \multicolumn{2}{|c|}{158.32} \\
\hline \multicolumn{2}{|c|}{ Xenon Thermal Conductivity $(\mathrm{W} /(\mathrm{m}-\mathrm{K}))$} & \multicolumn{2}{|c|}{$-0.0002769+2.088 \mathrm{E}-5 \mathrm{~T}-5.323 \mathrm{E}-9 \mathrm{~T}^{2}$} \\
\hline \multicolumn{2}{|c|}{ Xenon Viscosity $(\mathrm{kg} /(\mathrm{m}-\mathrm{s}))$} & \multicolumn{2}{|c|}{$-1.167 \mathrm{E}-6+8.796 \mathrm{E}-8 \mathrm{~T}-2.243 \mathrm{E}-11 \mathrm{~T}^{2}$} \\
\hline \multicolumn{2}{|c|}{ Xenon Molecular Weight (kg/kgmol) } & \multicolumn{2}{|c|}{131.293} \\
\hline \multicolumn{2}{|c|}{ Aluminum Density $\left(\mathrm{kg} / \mathrm{m}^{3}\right)$} & \multicolumn{2}{|c|}{2719} \\
\hline \multicolumn{2}{|c|}{ Aluminum Specific Heat $(\mathrm{J} /(\mathrm{kg}-\mathrm{K}))$} & \multicolumn{2}{|c|}{871} \\
\hline \multicolumn{2}{|c|}{ Aluminum Thermal Conductivity $(\mathrm{W} /(\mathrm{m}-\mathrm{K}))$} & \multicolumn{2}{|c|}{202.4} \\
\hline \multirow[t]{6}{*}{ Inlet } & \multicolumn{3}{|l|}{ Far-field pressure } \\
\hline & \multicolumn{2}{|l|}{ Gauge pressure } & $0 \mathrm{~Pa}$ \\
\hline & \multicolumn{2}{|l|}{ Mach number } & 0.7509739 \\
\hline & \multicolumn{2}{|c|}{ Temperature } & $1050 \mathrm{~K}$ \\
\hline & \multicolumn{2}{|c|}{ Axial-component of flow direction } & 1 \\
\hline & Radial-component of flow dire & & 0 \\
\hline
\end{tabular}




\begin{tabular}{|c|c|c|c|c|}
\hline & \multicolumn{3}{|c|}{ Tangential-component of flow direction } & 0 \\
\hline & \multicolumn{3}{|c|}{ Turbulent intensity } & $1 \%$ \\
\hline & \multicolumn{3}{|c|}{ Turbulent viscosity ratio } & $1 \%$ \\
\hline Target $1-5$ & \multicolumn{3}{|c|}{ Moving wall with absolute, rotational motion } & \\
\hline & \multicolumn{3}{|c|}{ Rotation-Axis Origin } & $(0,0,0)$ \\
\hline & \multicolumn{3}{|c|}{ Rotation-Axis Direction } & $(1,0,0)$ \\
\hline & \multicolumn{3}{|c|}{ Rotational Speed } & $1570.796 \mathrm{rad} / \mathrm{s}$ \\
\hline & \multicolumn{3}{|c|}{ No slip } & \\
\hline & \multicolumn{3}{|c|}{ Roughness height } & $0 \mathrm{~m}$ \\
\hline & \multicolumn{3}{|c|}{ Roughness constant } & 0.5 \\
\hline & \multicolumn{3}{|c|}{ Temperature } & $20 \mathrm{~K}$ \\
\hline & \multicolumn{3}{|c|}{ Heat generation rate } & $0 \mathrm{~W} / \mathrm{m}^{3}$ \\
\hline & \multicolumn{3}{|c|}{ Material } & Aluminum \\
\hline Outer Wall & \multicolumn{4}{|c|}{ Stationary wall } \\
\hline & \multicolumn{3}{|c|}{ Specified shear x-component } & $0 \mathrm{~Pa}$ \\
\hline & \multicolumn{3}{|c|}{ Specified shear y-component } & $0 \mathrm{~Pa}$ \\
\hline & \multicolumn{3}{|c|}{ Specified shear z-component } & $0 \mathrm{~Pa}$ \\
\hline & \multicolumn{3}{|c|}{ Roughness height } & $0 \mathrm{~m}$ \\
\hline & \multicolumn{3}{|c|}{ Roughness constant } & 0.5 \\
\hline & \multicolumn{3}{|c|}{ Heat flux } & $0 \mathrm{~W} / \mathrm{m}^{2}$ \\
\hline & \multicolumn{3}{|c|}{ Heat generation rate } & $0 \mathrm{~W} / \mathrm{m}^{3}$ \\
\hline & \multicolumn{3}{|c|}{ Material } & Aluminum \\
\hline Outlet & \multicolumn{4}{|c|}{ Pressure outlet } \\
\hline & \multicolumn{3}{|c|}{ Gauge pressure } & $0 \mathrm{~Pa}$ \\
\hline & \multicolumn{3}{|c|}{ Backflow direction specification method } & Normal to boundary \\
\hline & Backflow & urbulent intensity & & $1 \%$ \\
\hline & Backflow & urbulent viscosity ratio & & 1 \\
\hline & Backflow & total temperature & & $1247.39 \mathrm{~K}$ \\
\hline Operating $\mathrm{p}$ & essure & $6666.118 \mathrm{~Pa}$ & & \\
\hline Solver & & Density-based, Implicit & & \\
\hline Flux Type & & Roe-FDS & & \\
\hline Discretizati & Scheme & Gradient & & en-Gauss Node Based \\
\hline & & Flow & & t order upwind \\
\hline & & Turbulent Kinetic Energy & & order upwind \\
\hline & & Specified Dissipation Rate & Fir & order upwind \\
\hline Turbulent $\mathrm{K}$ & netic Eners & Relaxation Factor & 0.8 & \\
\hline Specified D & sipation R & te Relaxation Factor & 0.8 & \\
\hline Turbulent V & scosity Rel & xation Factor & 1 & \\
\hline Solid Relax & ion Factor & & 1 & \\
\hline Courant $\mathrm{Nu}$ & ber & & 5 & \\
\hline Solution Lir & & & $\mathrm{De}$ & ault \\
\hline Residual M & nitor & Absolute convergence criteria & & $1 \mathrm{e}-4$ \\
\hline & & Iterations to store & & 10000 \\
\hline
\end{tabular}




\begin{tabular}{|l|l|l|}
\hline Solution Initialization & Standard, absolute & Compute from inlet \\
\hline Initial Values & Gauge Pressure & $0 \mathrm{~Pa}$ \\
\hline & X Velocity & $250 \mathrm{~m} / \mathrm{s}$ \\
\hline & Y Velocity & $0 \mathrm{~m} / \mathrm{s}$ \\
\hline & Z Velocity & $0 \mathrm{~m} / \mathrm{s}$ \\
\hline & Turbulent Kinetic Energy & $9.37473 \mathrm{~m}^{2} / \mathrm{s}^{2}$ \\
\hline & Specific Dissipation Rate & $52213.611 / \mathrm{s}$ \\
\hline & Temperature & $1050 \mathrm{~K}$ \\
\hline & \multicolumn{2}{|l}{} \\
\hline & & 30000 \\
\hline
\end{tabular}


Appendix B: Additional Plots of 2D, Axisymmetric Swirl, Transient Simulation of Target in Drift Region

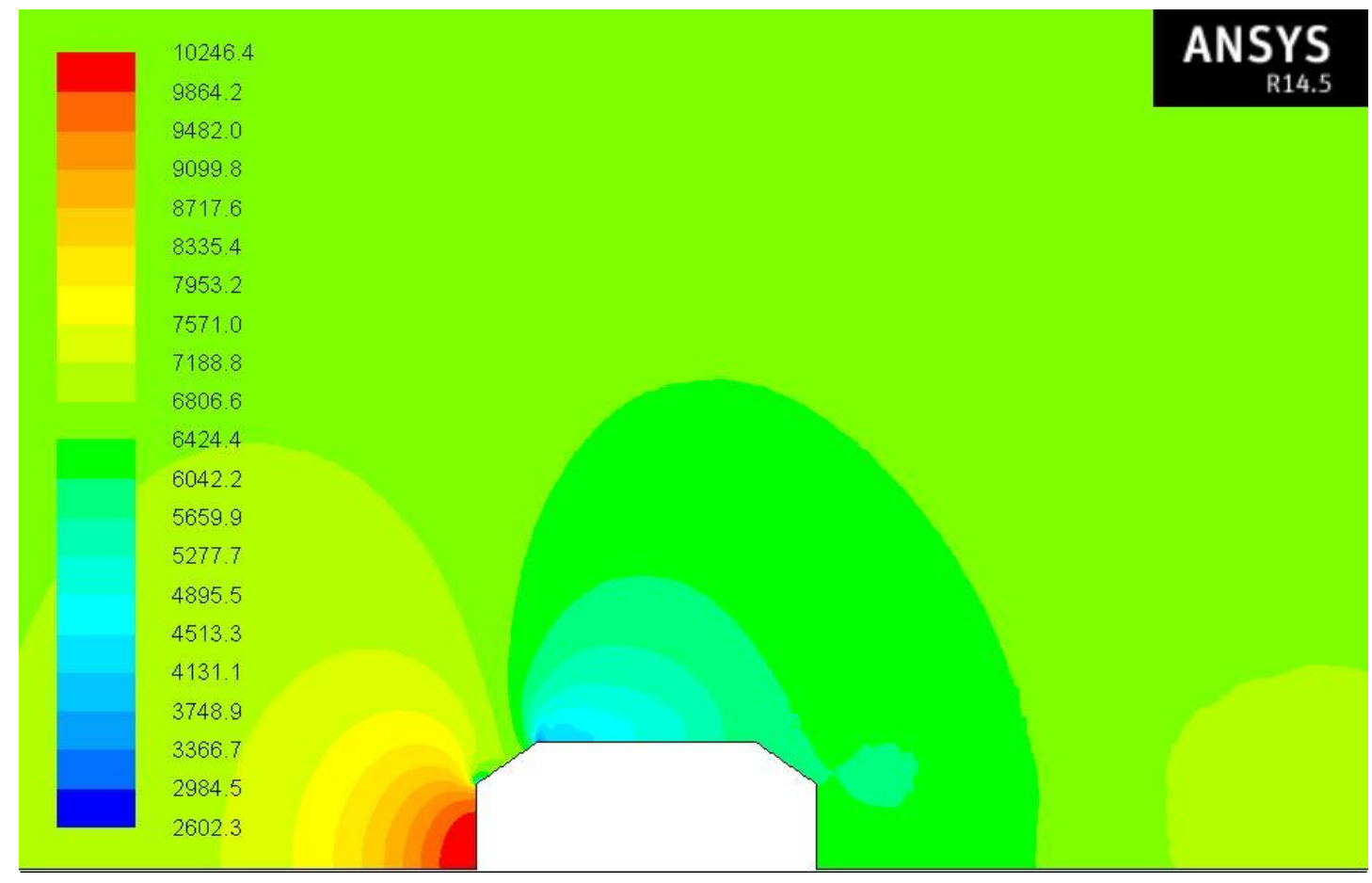

Figure 69. Pressure Contour of 2D, Axisymmetric Swirl Flow over Target in Drift Region at 1.0E-3 s

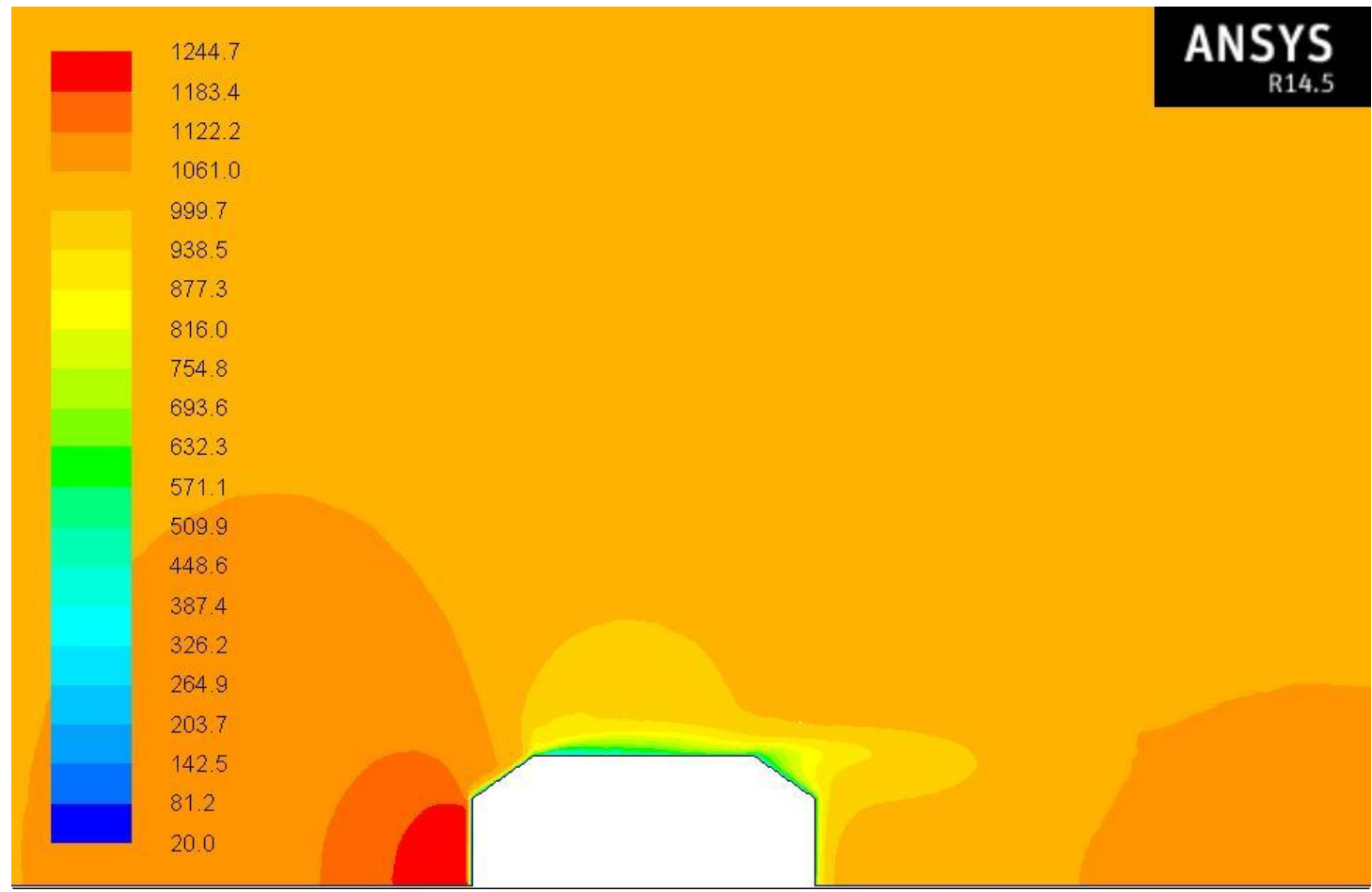

Figure 70. Temperature Contour of 2D, Axisymmetric Swirl Flow over Target in Drift Region at 1.0E-3 s 


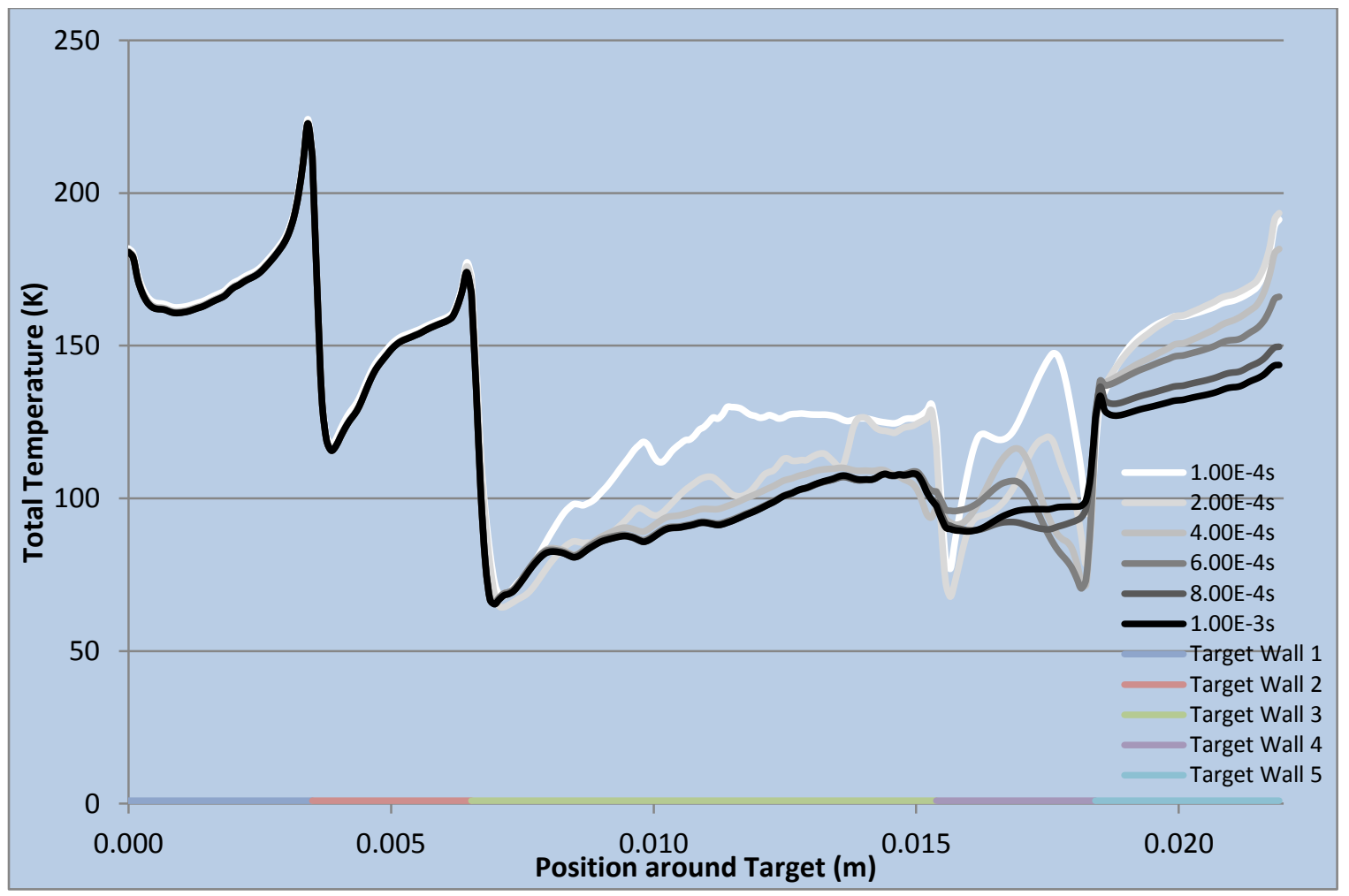

Figure 71. Total Temperature around Target in Drift Region from 2D, Axisymmetric Swirl Model

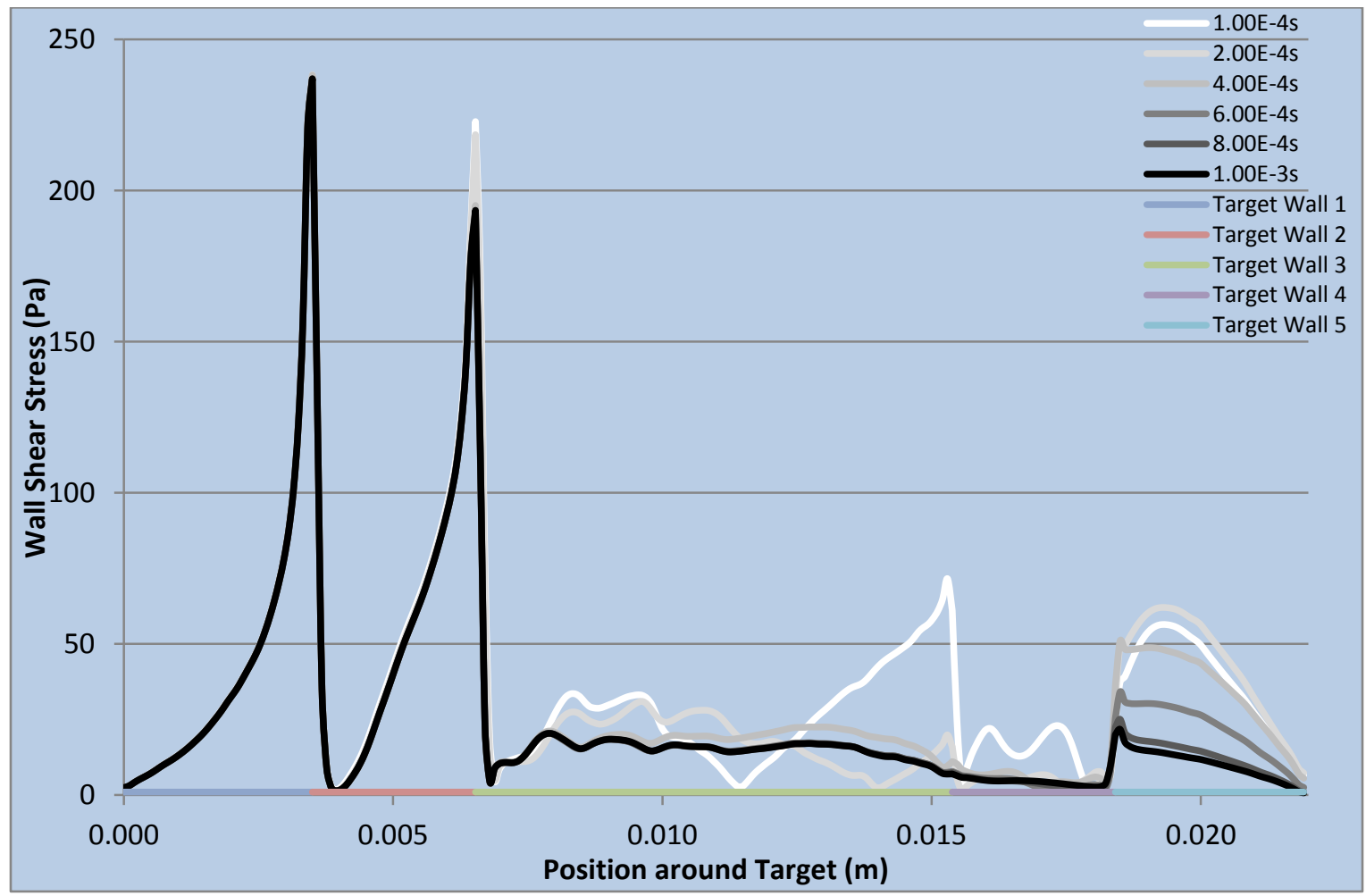

Figure 72. Wall Shear Stress around Target in Drift Region from 2D, Axisymmetric Swirl Model 


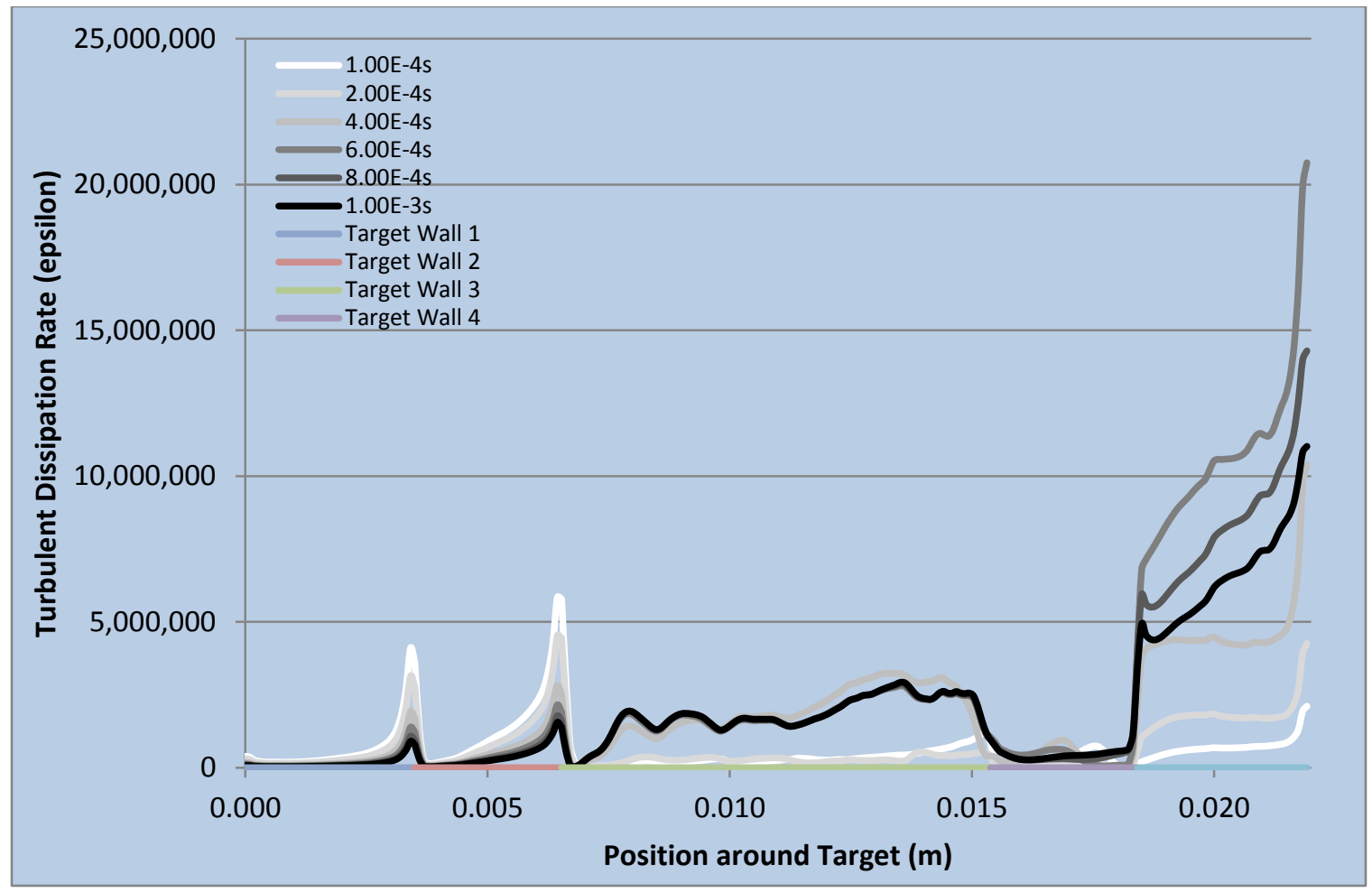

Figure 73. Turbulent Dissipation Rate around Target in Drift Region from 2D, Axisymmetric Swirl Model

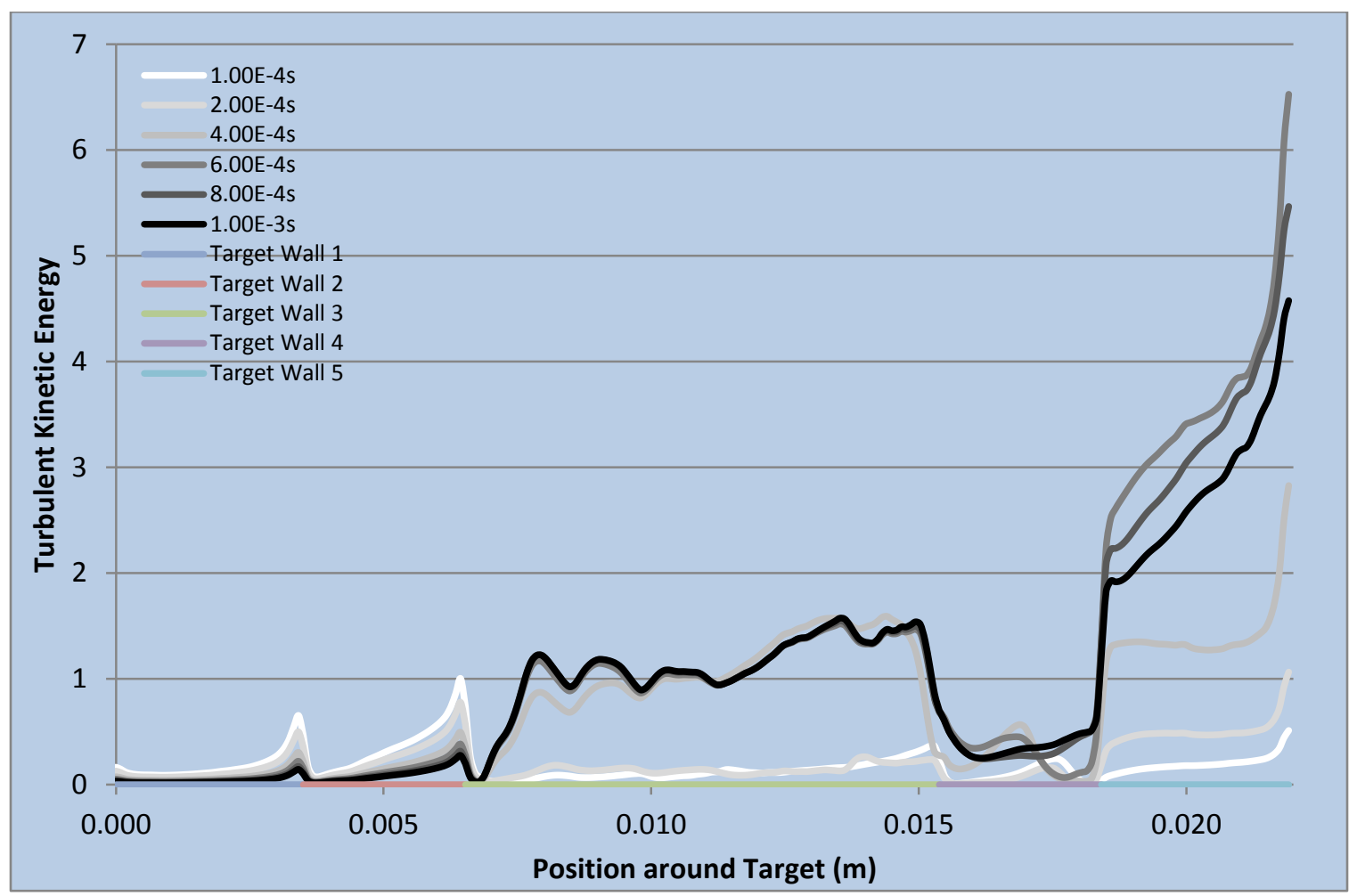

Figure 74. Turbulent Kinetic Energy around Target in Drift Region from 2D, Axisymmetric Swirl Model 


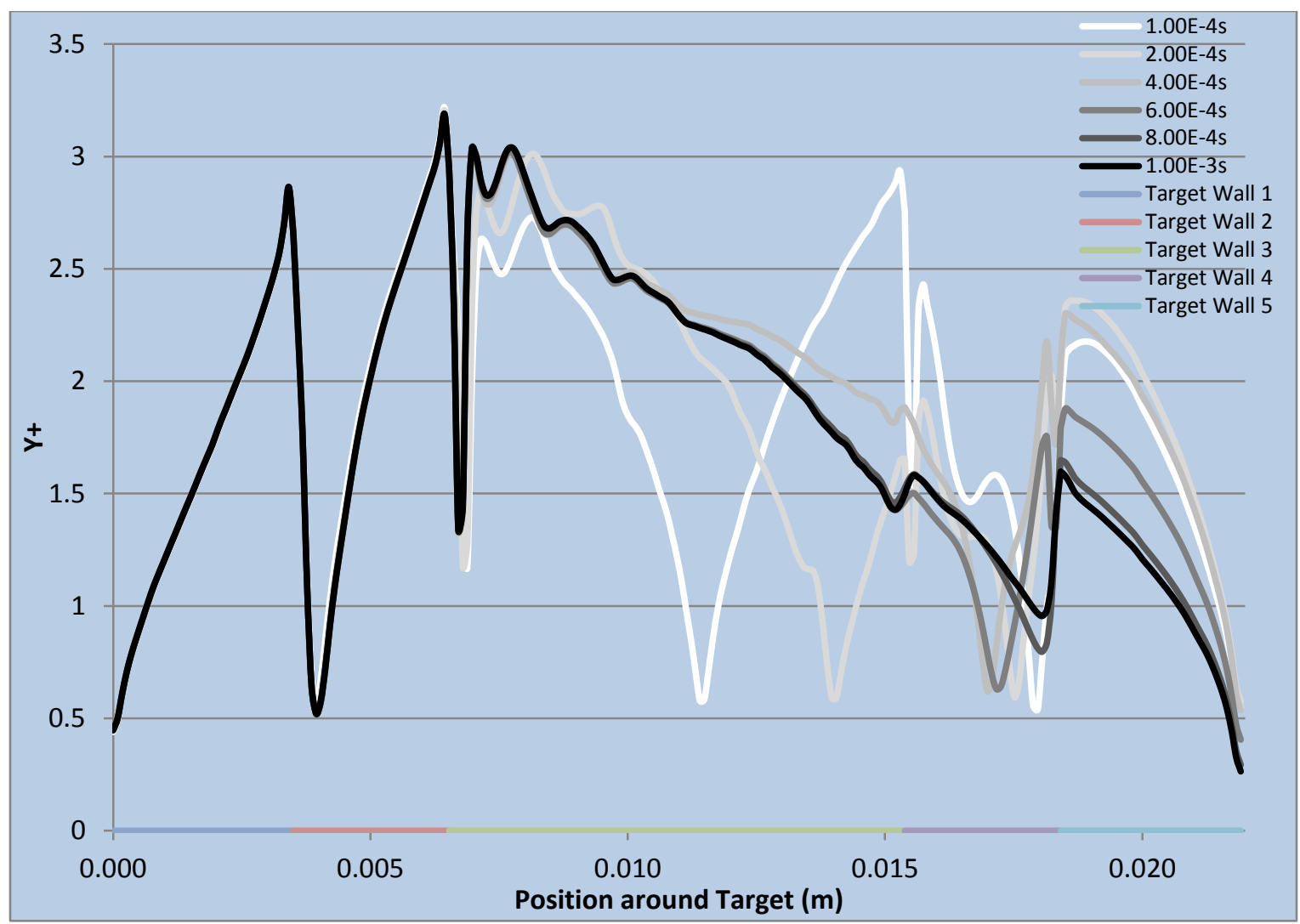

Figure 75. Y+ Values around Target in Drift Region from 2D, Axisymmetric Swirl Model 
Appendix C: Additional Plots of 3D, Steady-State Simulations of Target in Drift Region

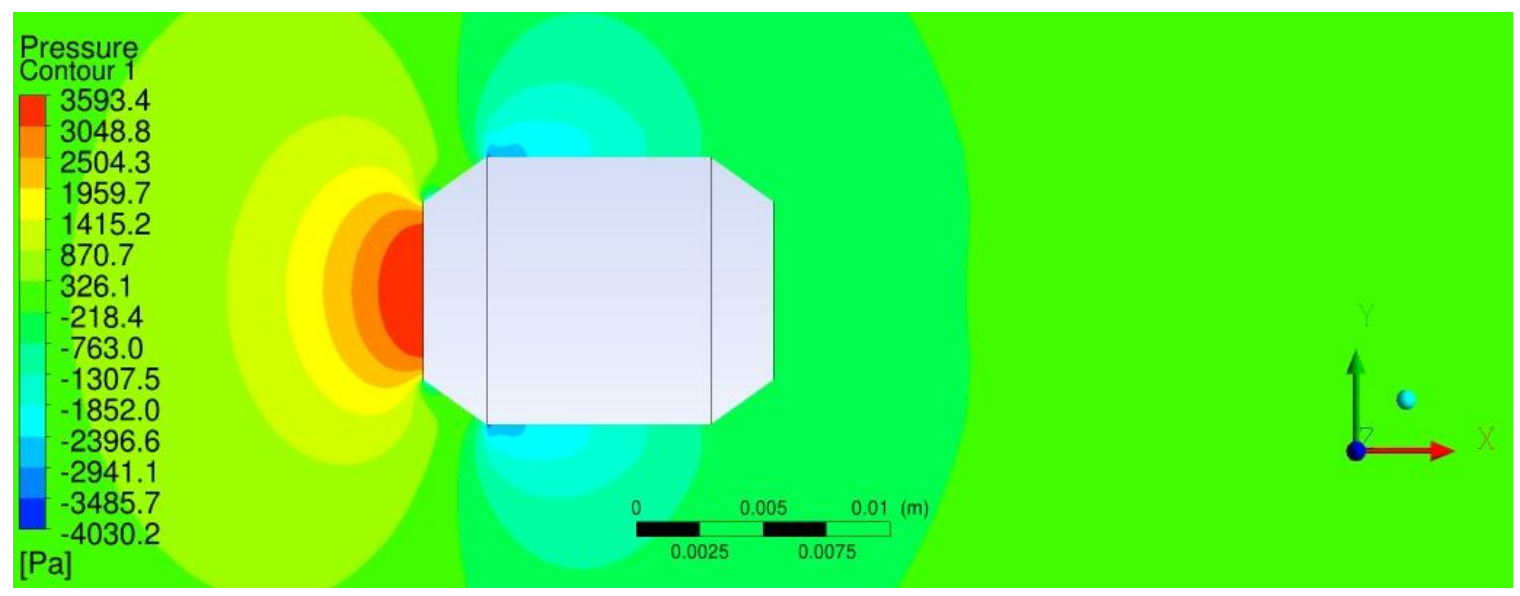

Figure 76. Pressure Contour of 3D, Steady-State Spinning Target in Drift Region

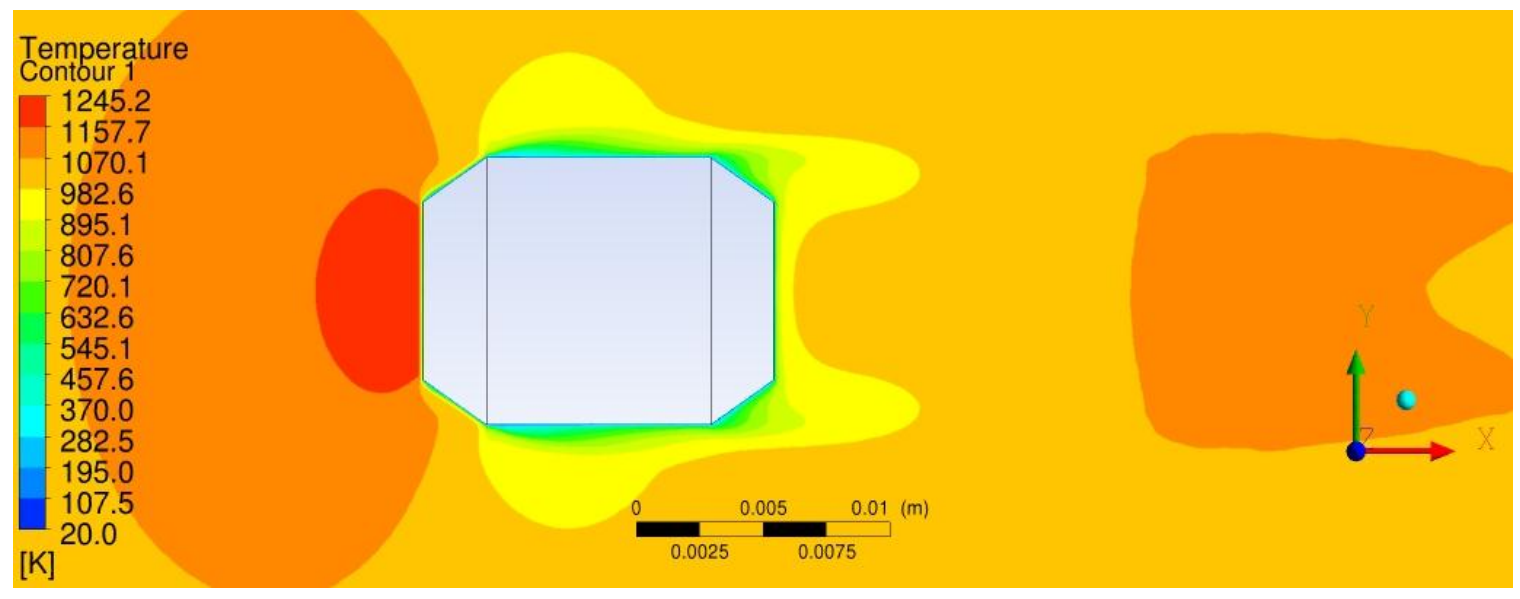

Figure 77. Temperature Contour of 3D, Steady-State Spinning Target in Drift Region 


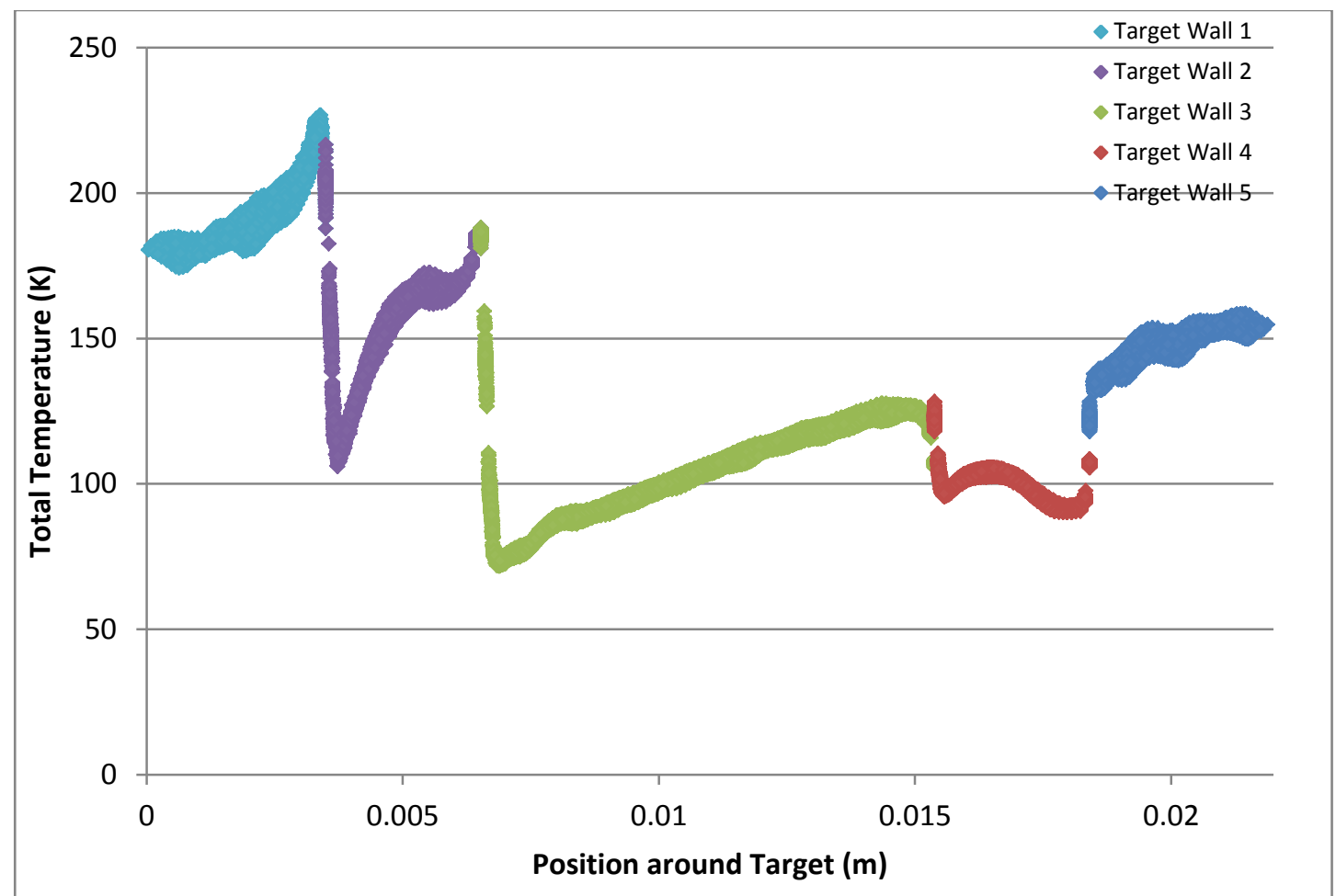

Figure 78. Total Temperature around Target in Drift Region from 3D, Steady-State Model

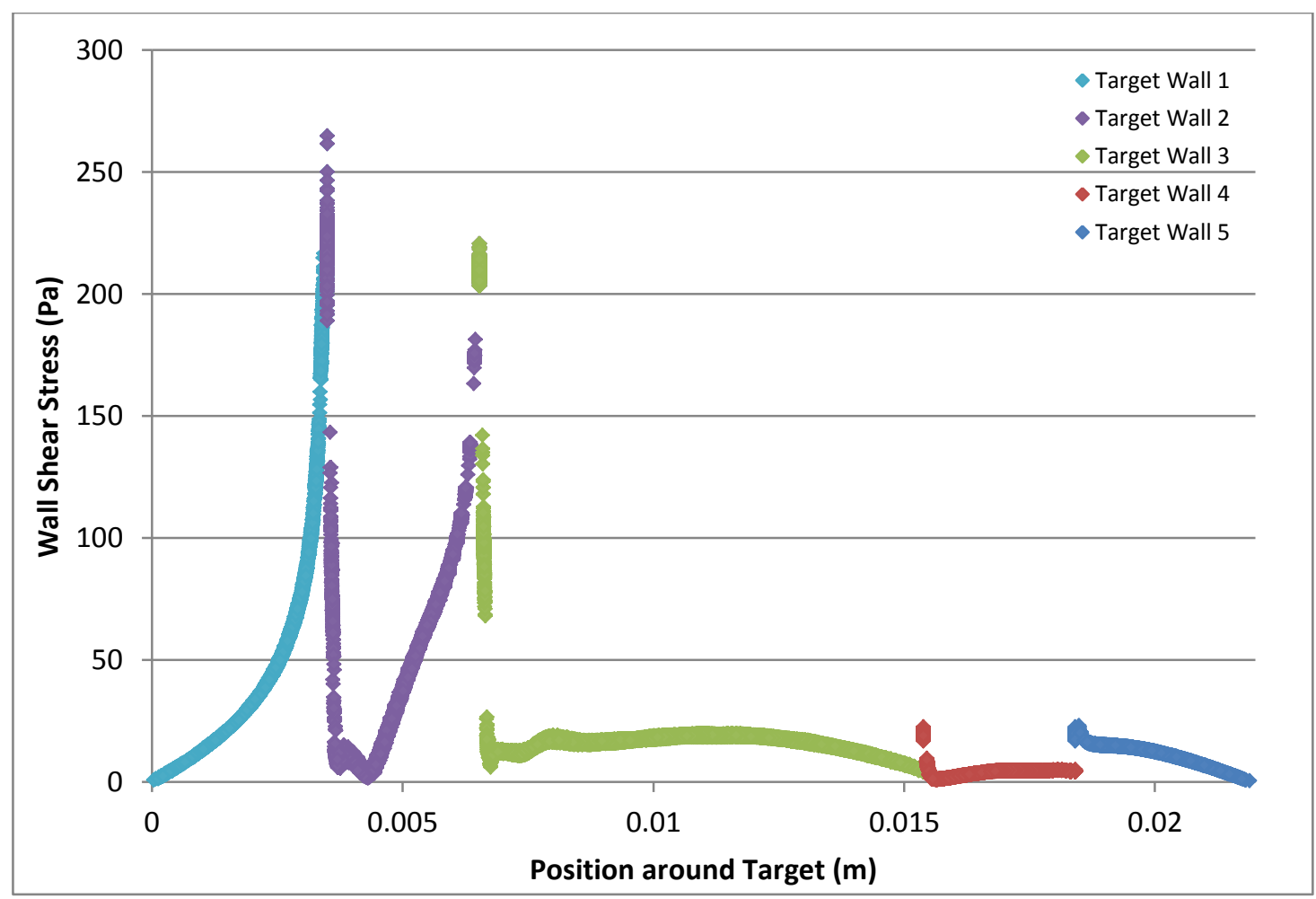

Figure 79. Wall Shear Stress around Target in Drift Region from 3D, Steady-State Model 


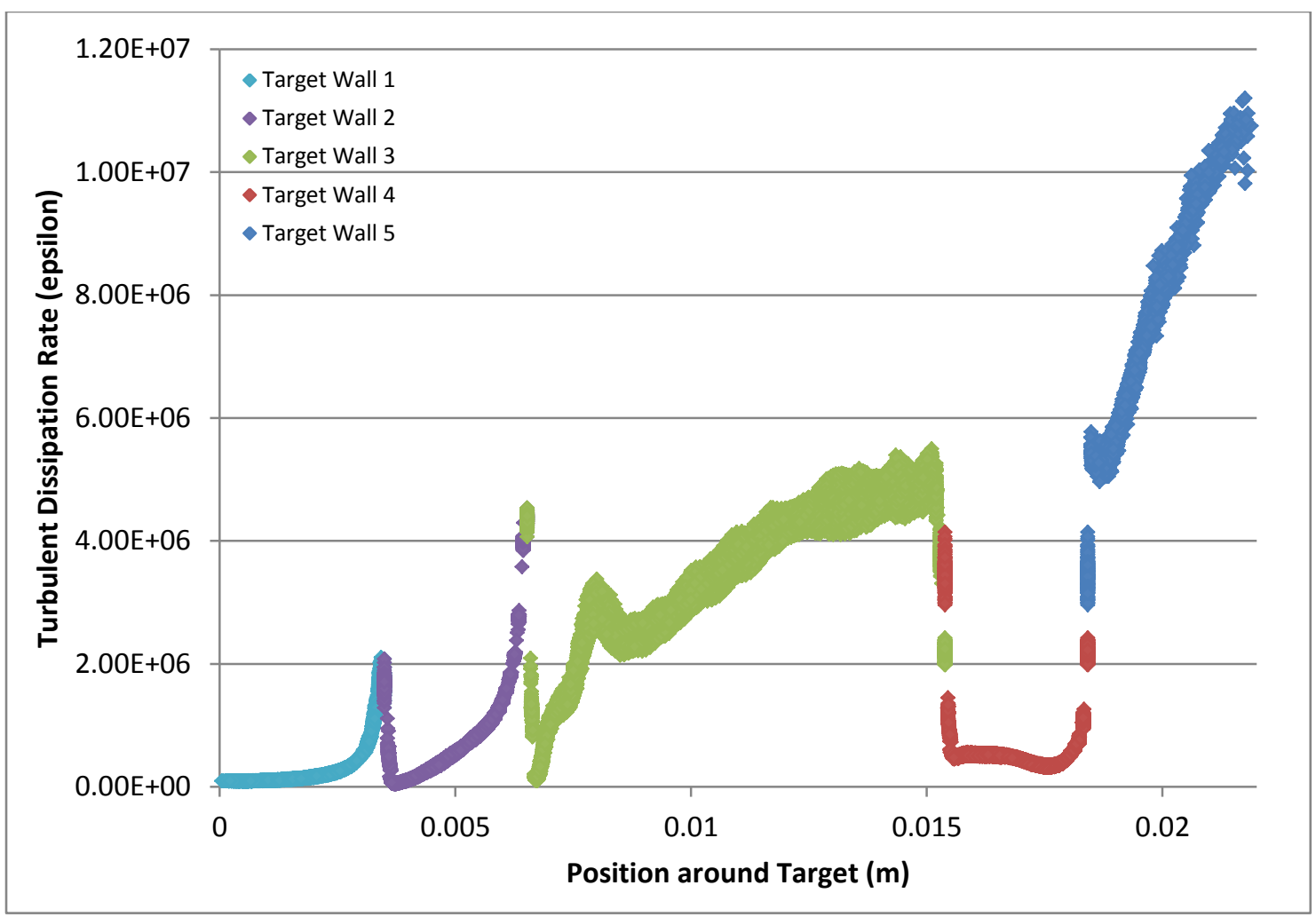

Figure 80. Turbulent Dissipation Rate around Target in Drift Region from 3D, Steady-State Model

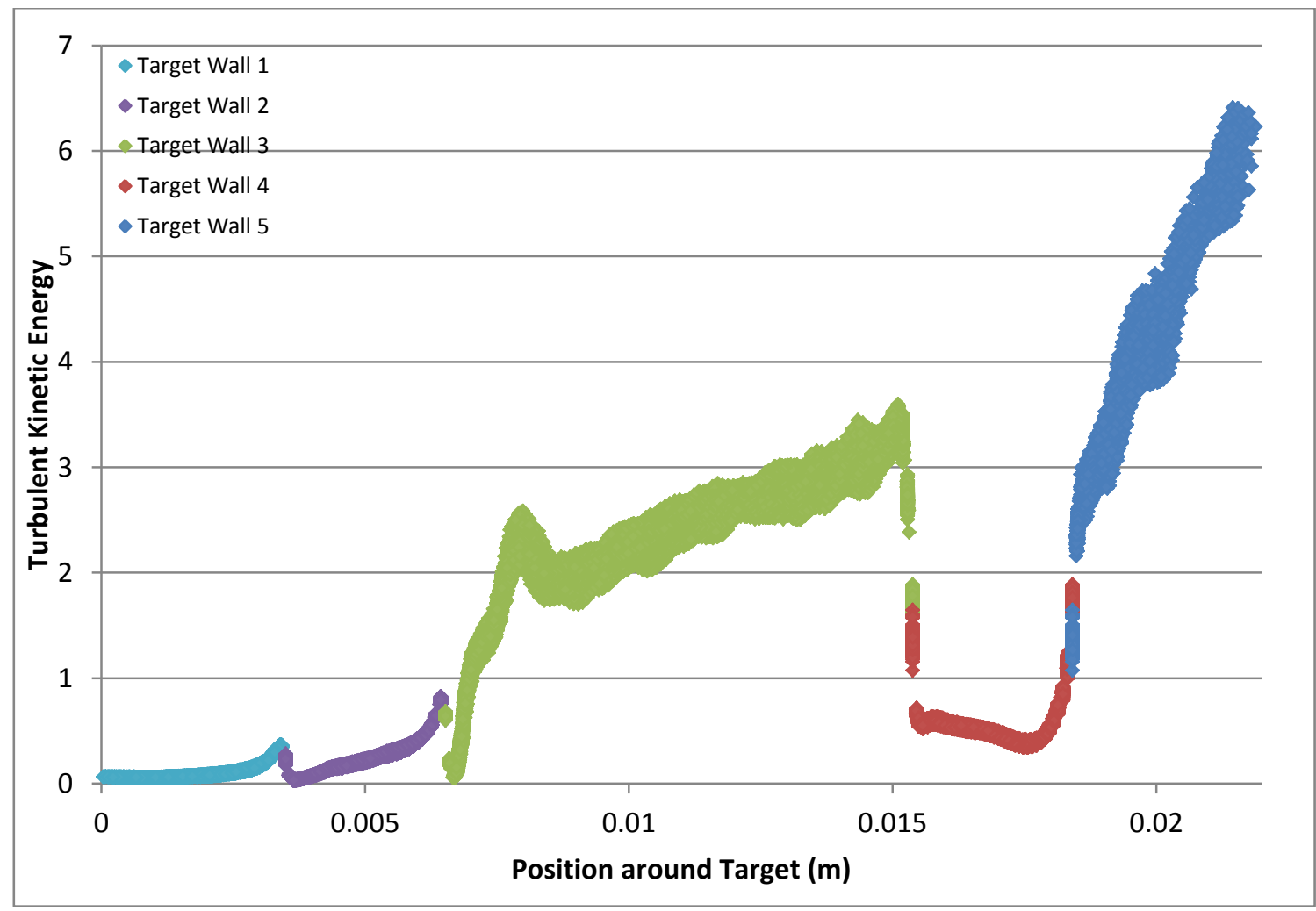

Figure 81. Turbulent Kinetic Energy around Target in Drift Region from 3D, Steady-State Model 


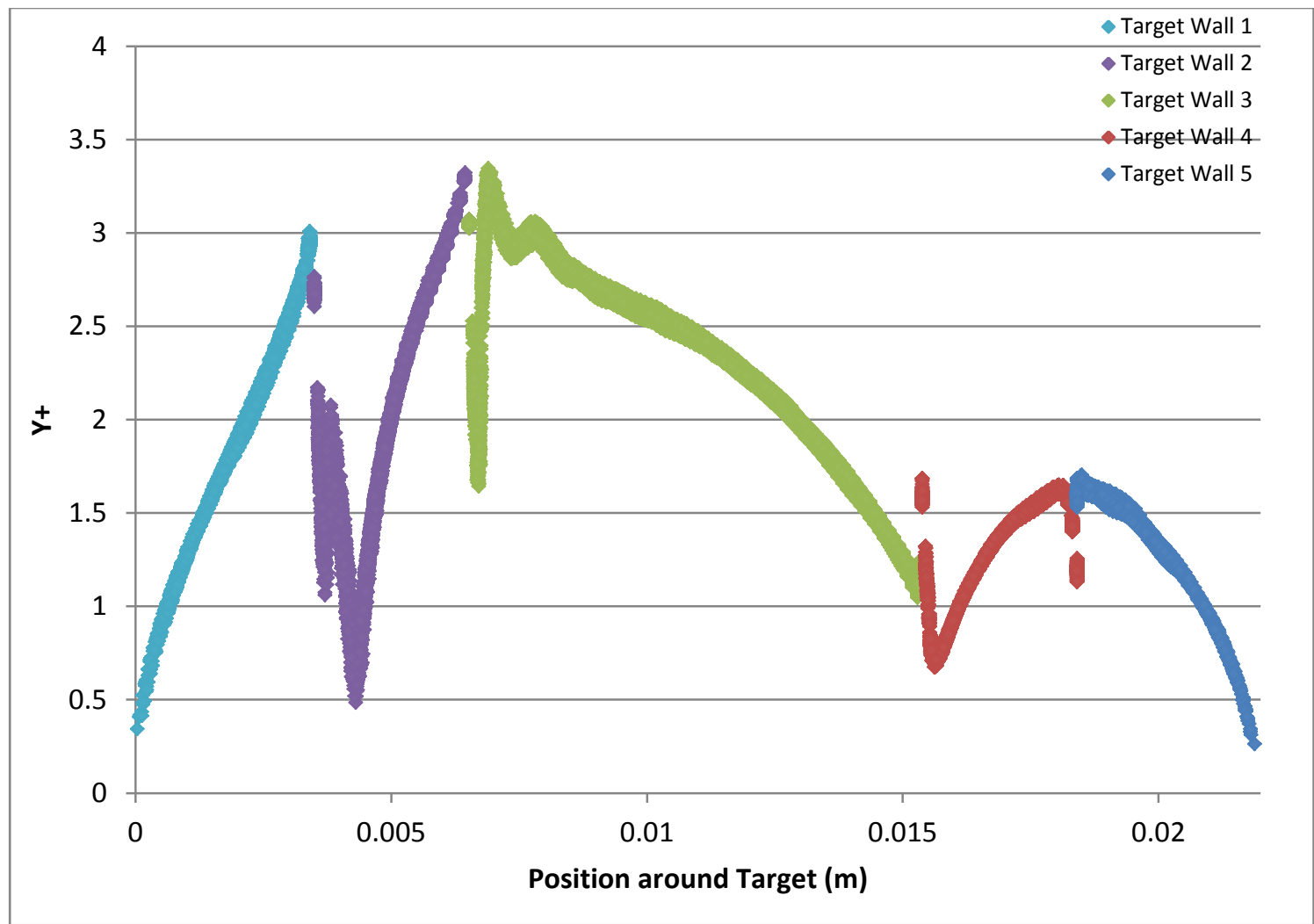

Figure 82. Y+ Values around Target in Drift Region from 3D, Steady-State Model

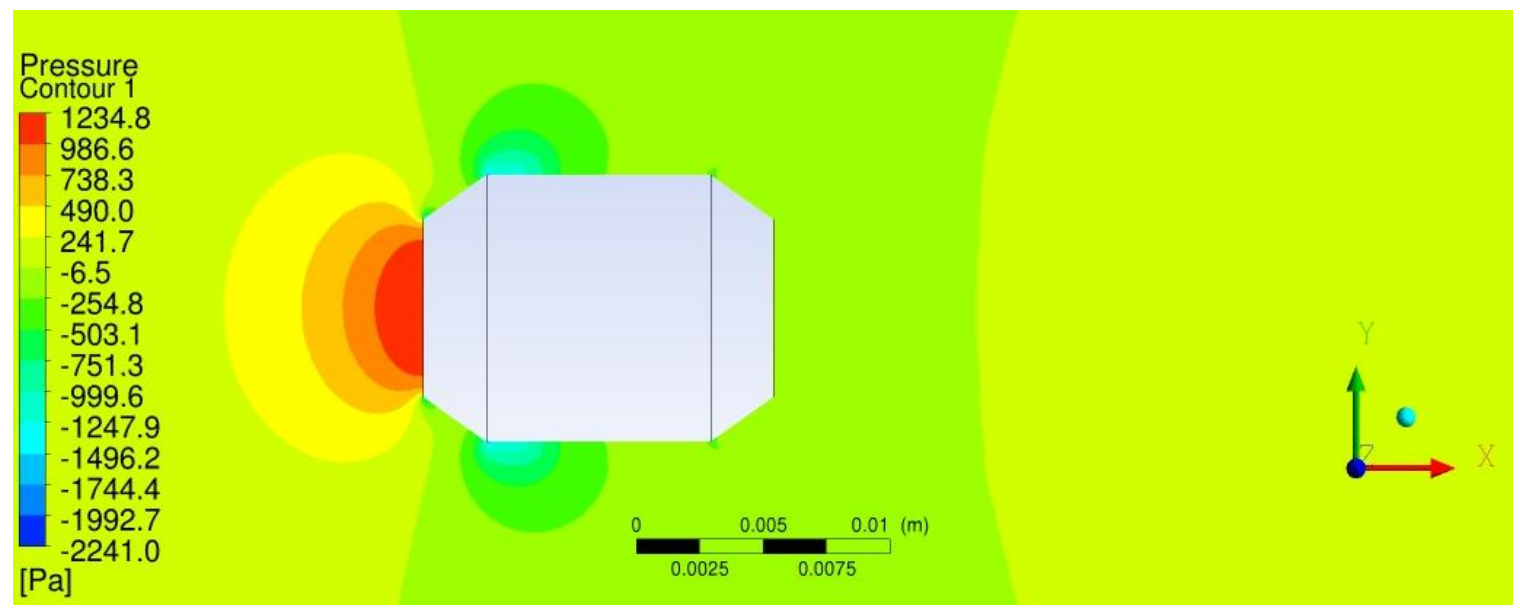

Figure 83. Pressure Contour of 3D, Steady-State, Spinning Target in Drift Region at a Reynolds Number of 2,400 


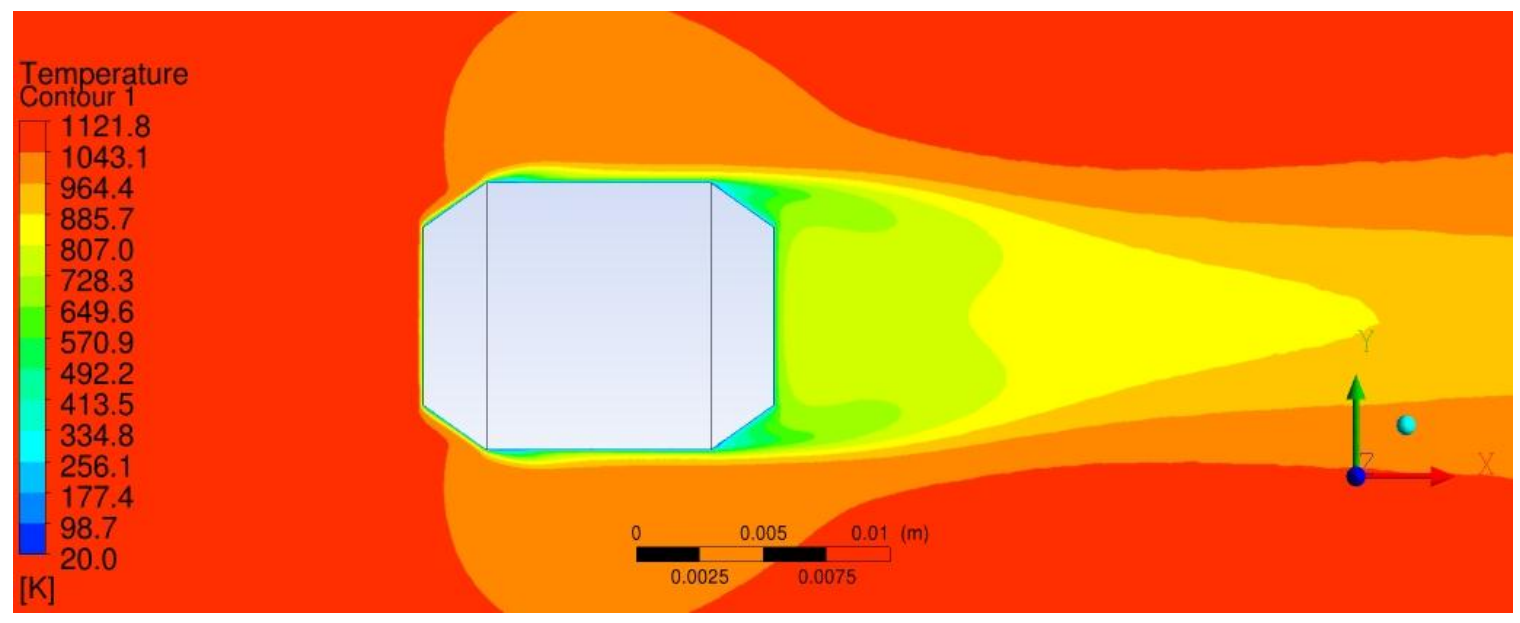

Figure 84. Temperature Contour of 3D, Steady-State, Spinning Target in Drift Region at a Reynolds Number of 2,400

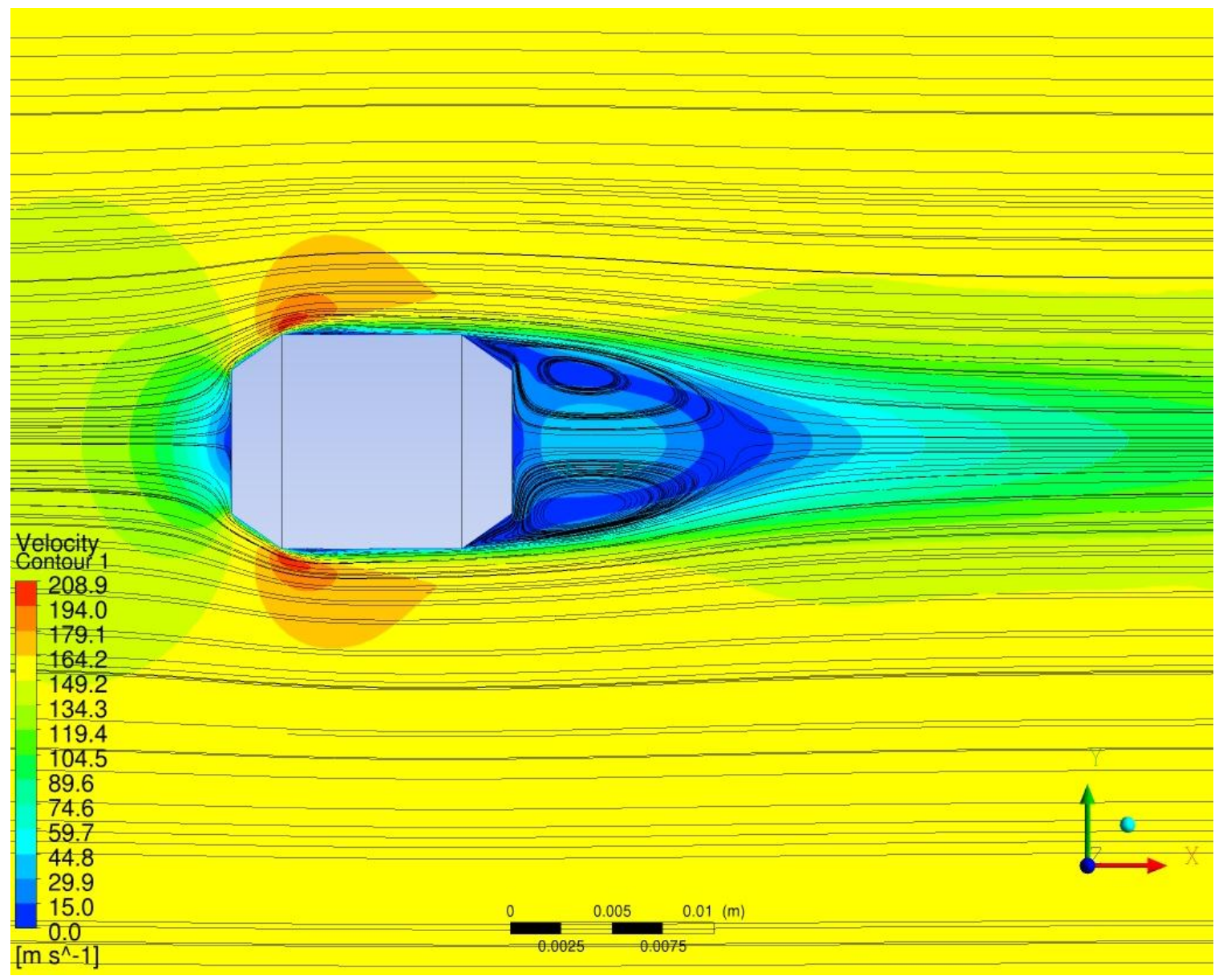

Figure 85. Velocity Contour with Streamlines of 3D, Steady-State, Spinning Target in Drift Region at a Reynolds Number of $\mathbf{2 , 4 0 0}$ 


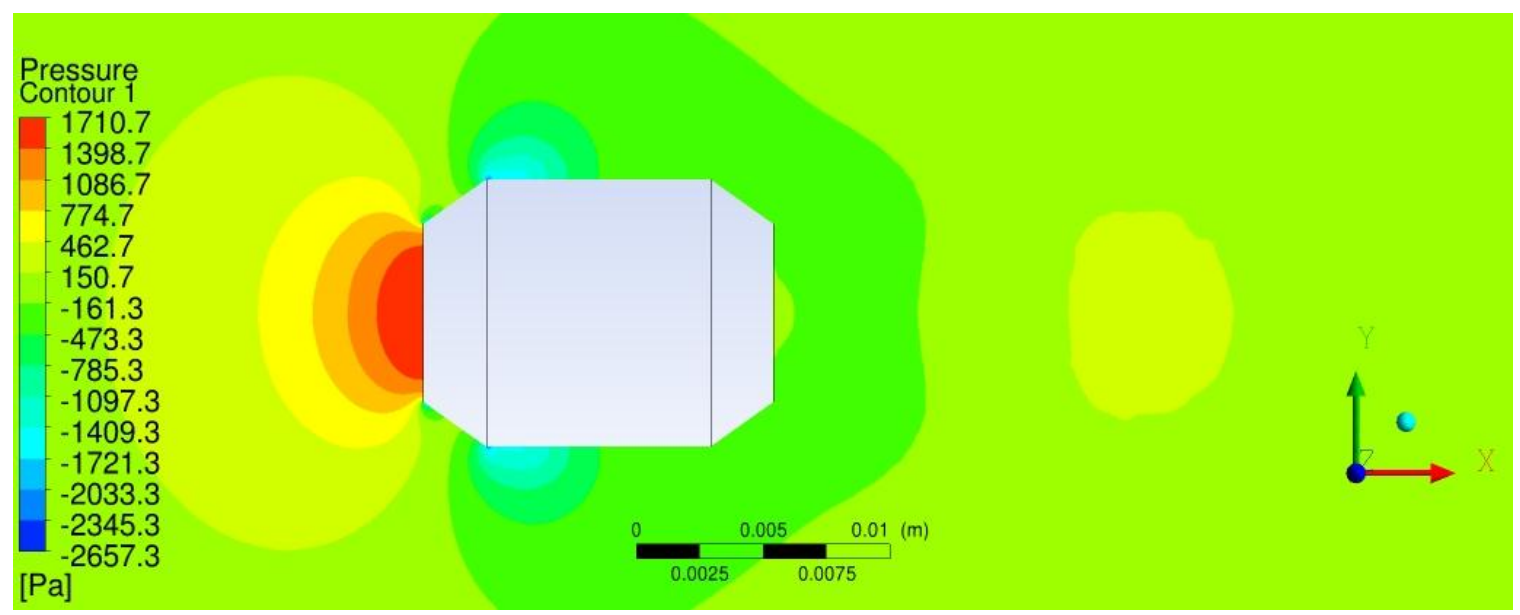

Figure 86. Pressure Contour of 3D, Steady-State, Spinning Target in Drift Region at a Reynolds Number of 2,800

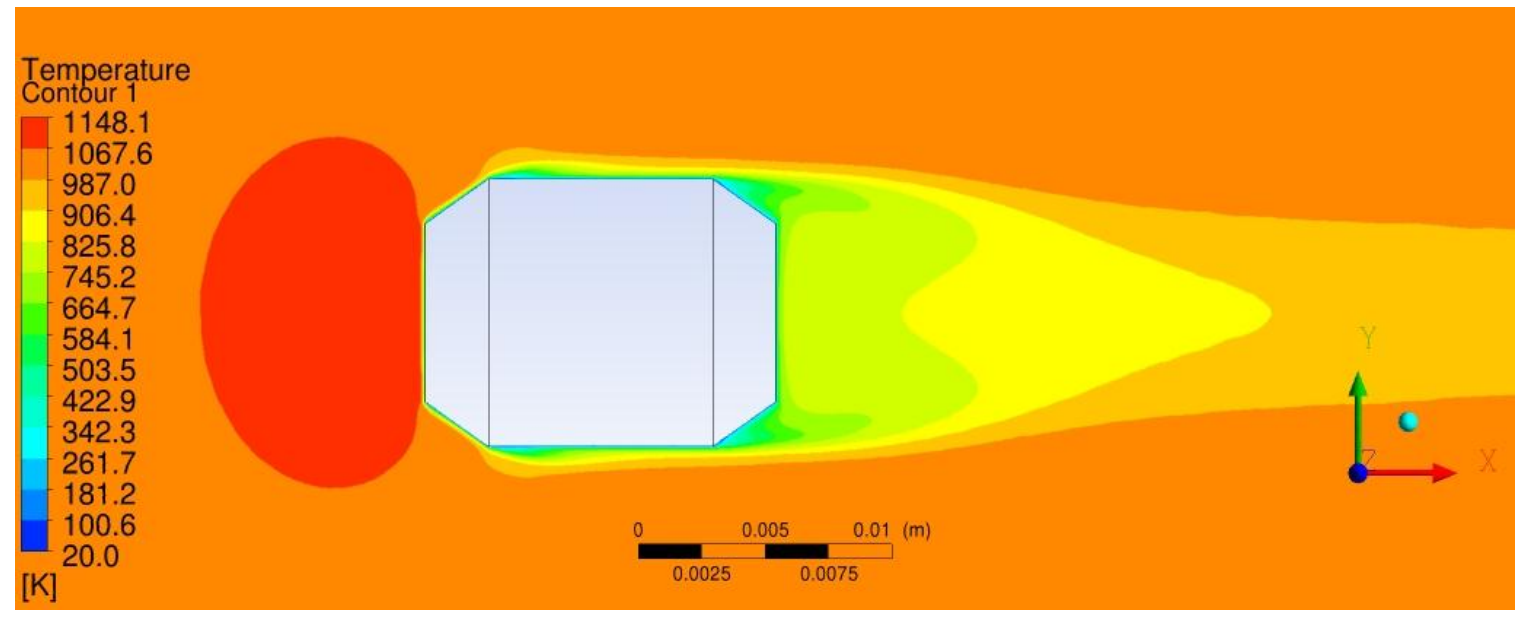

Figure 87. Temperature Contour of 3D, Steady-State, Spinning Target in Drift Region at a Reynolds Number of 2,800 


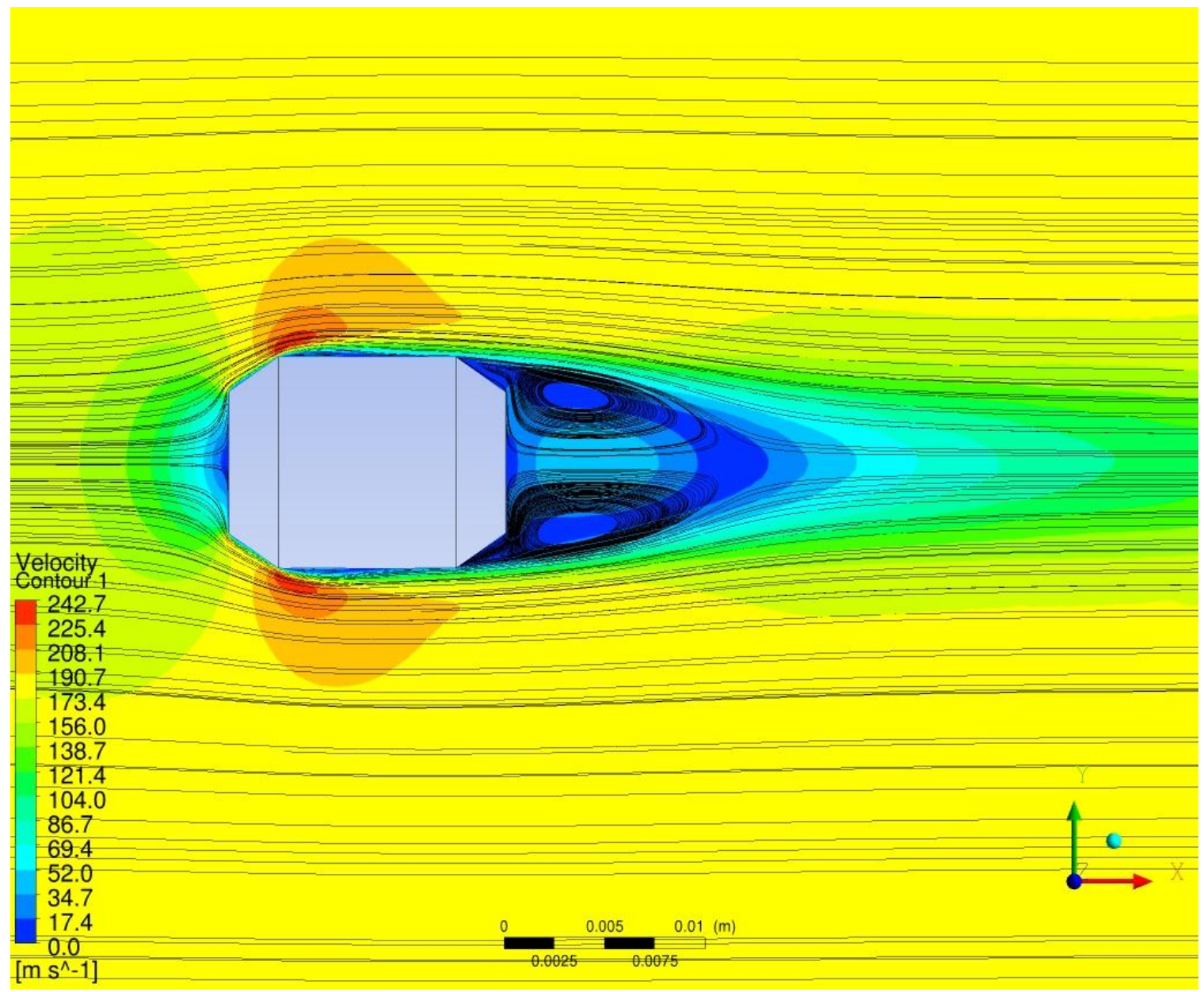

Figure 88. Velocity Contour with Streamlines of 3D, Steady-State, Spinning Target in Drift Region at a Reynolds Number of 2,800

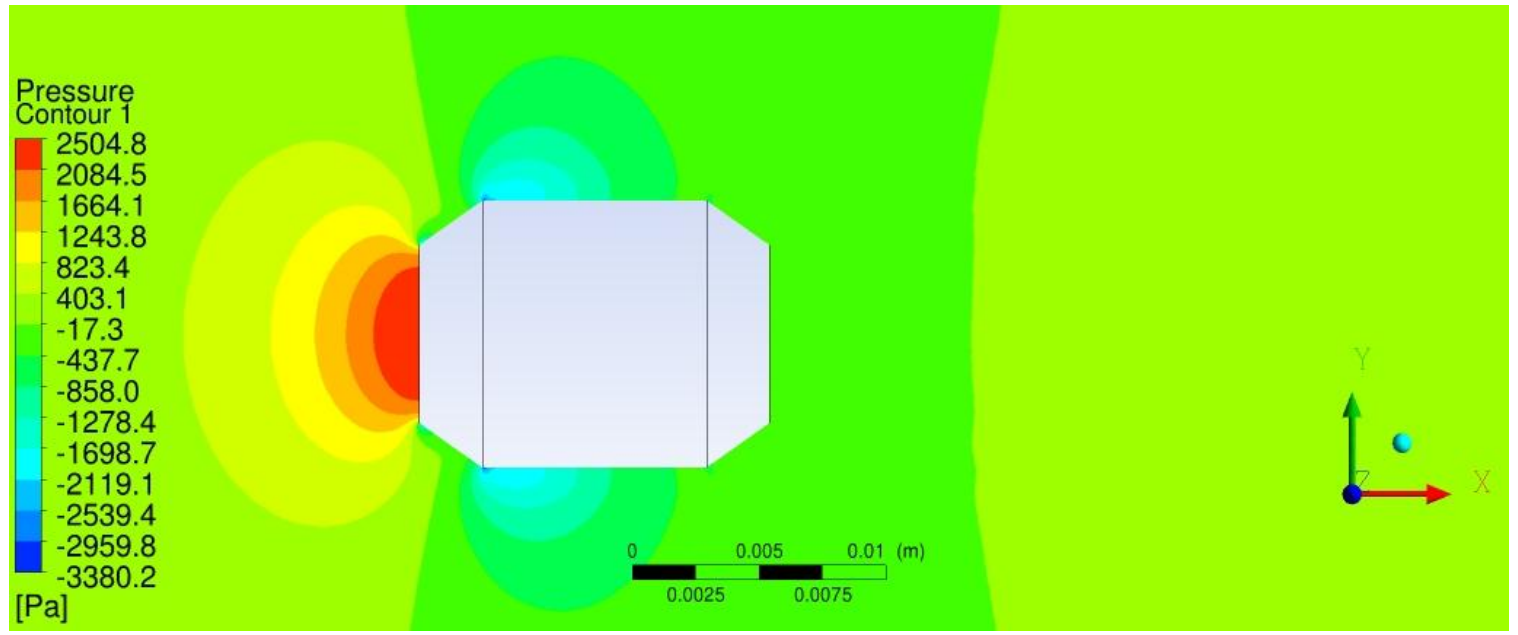

Figure 89. Pressure Contour of 3D, Steady-State, Spinning Target in Drift Region at a Reynolds Number of 3,200 


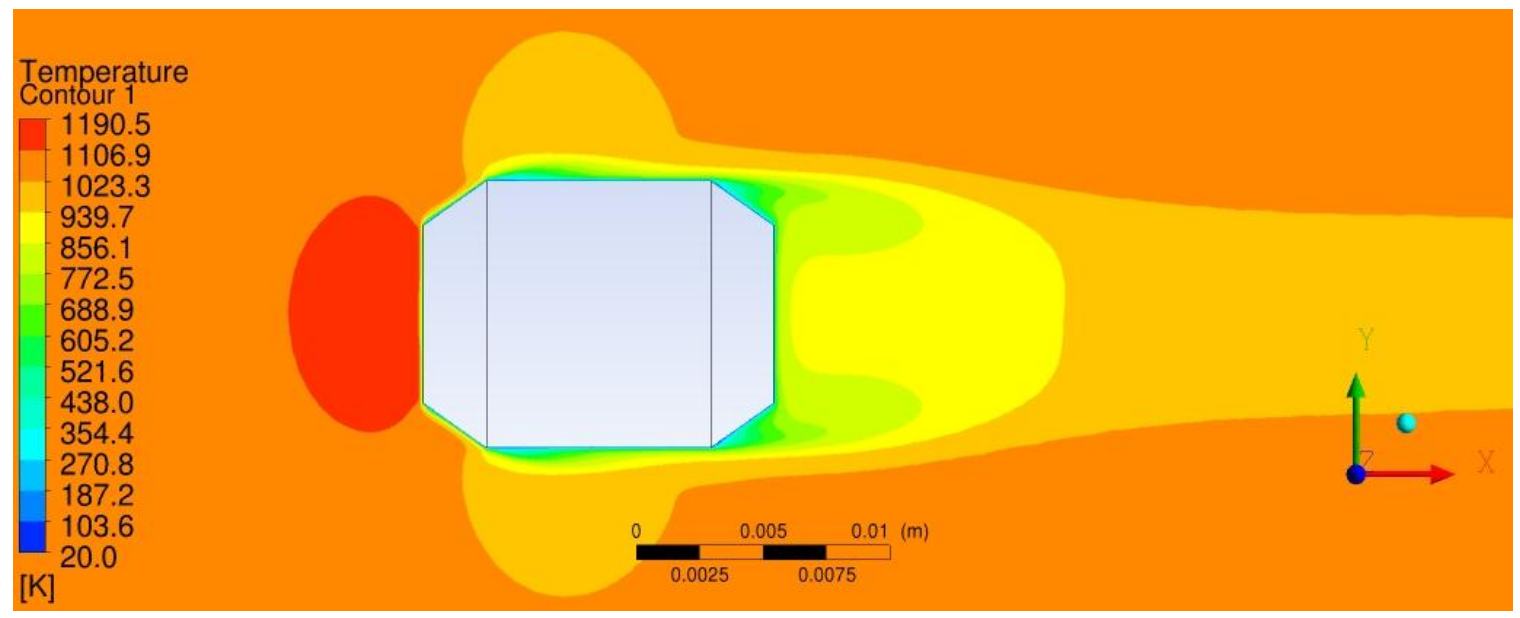

Figure 90. Temperature Contour of 3D, Steady-State, Spinning Target in Drift Region at a Reynolds Number of 3,200

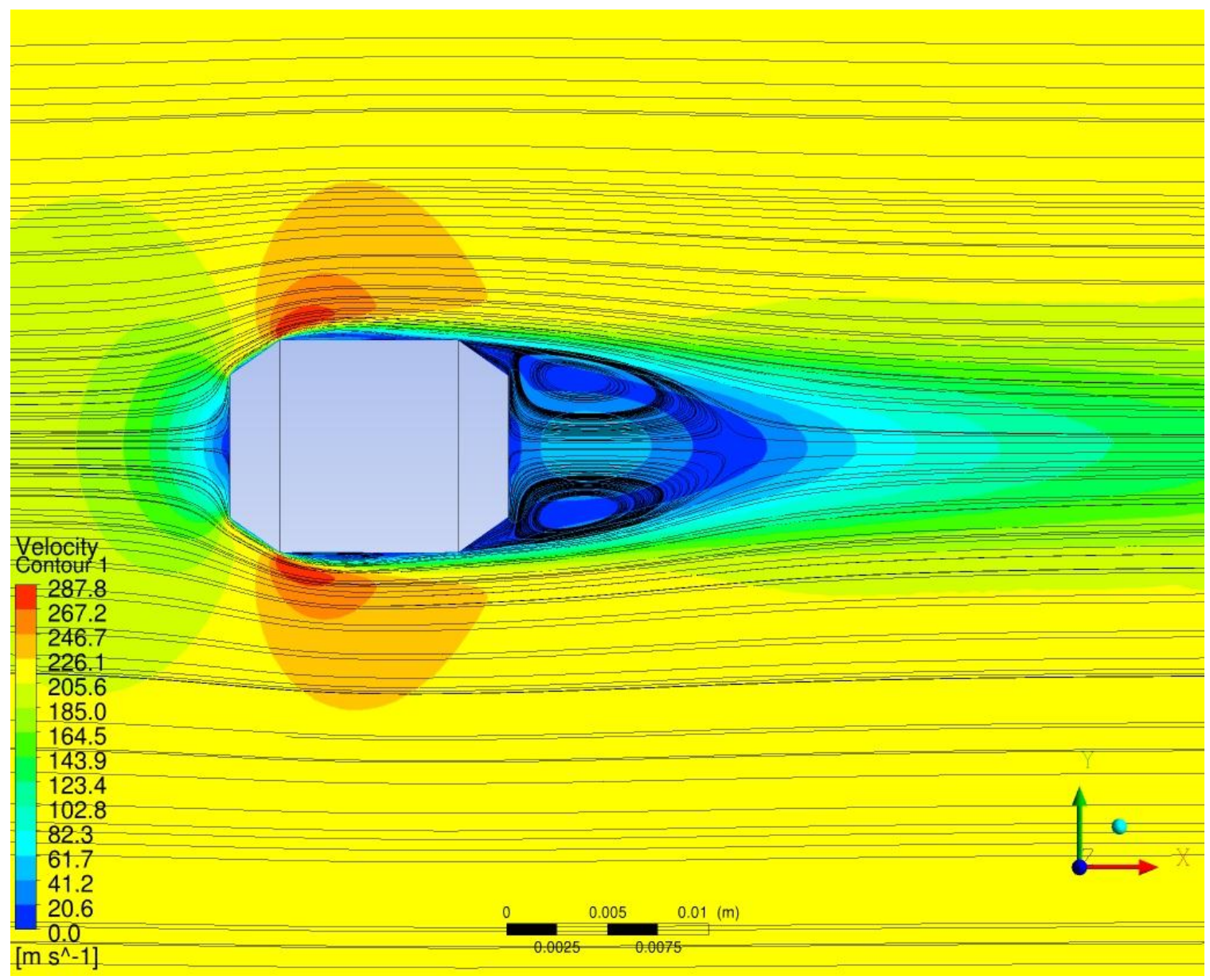

Figure 91. Velocity Contour with Streamlines of 3D, Steady-State, Spinning Target in Drift Region at a Reynolds Number of 3,200 


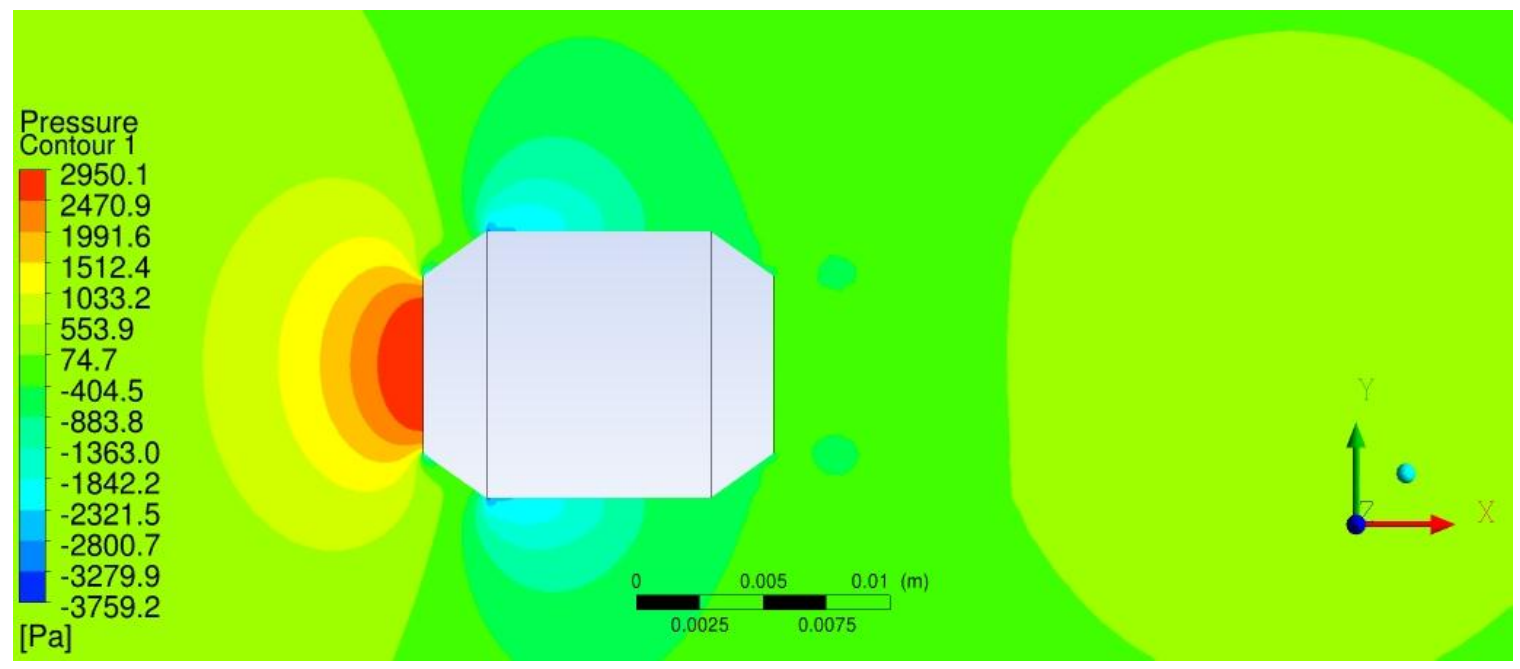

Figure 92. Pressure Contour of 3D, Steady-State, Spinning Target in Drift Region at a Reynolds Number of 3,600

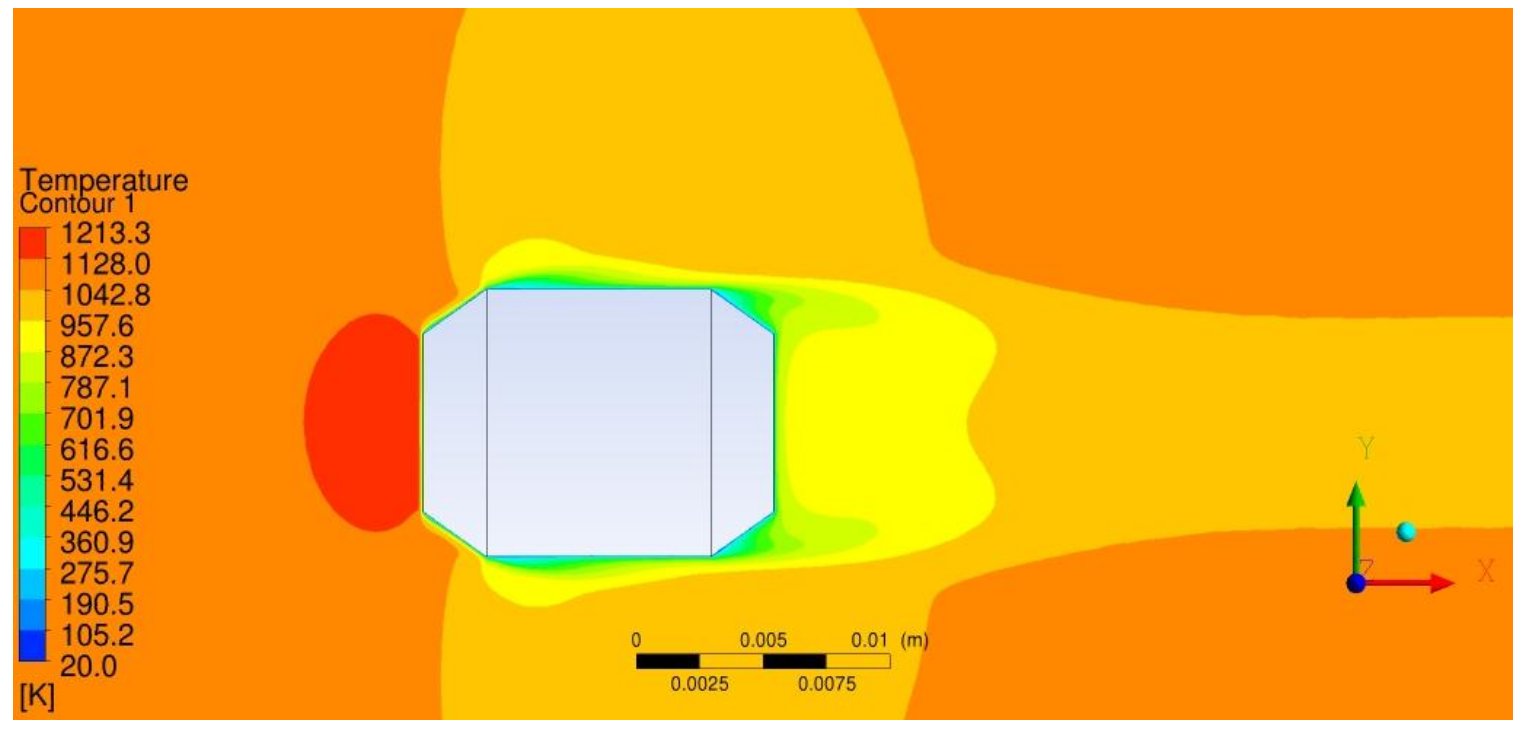

Figure 93. Temperature Contour of 3D, Steady-State, Spinning Target in Drift Region at a Reynolds Number of 3,600 


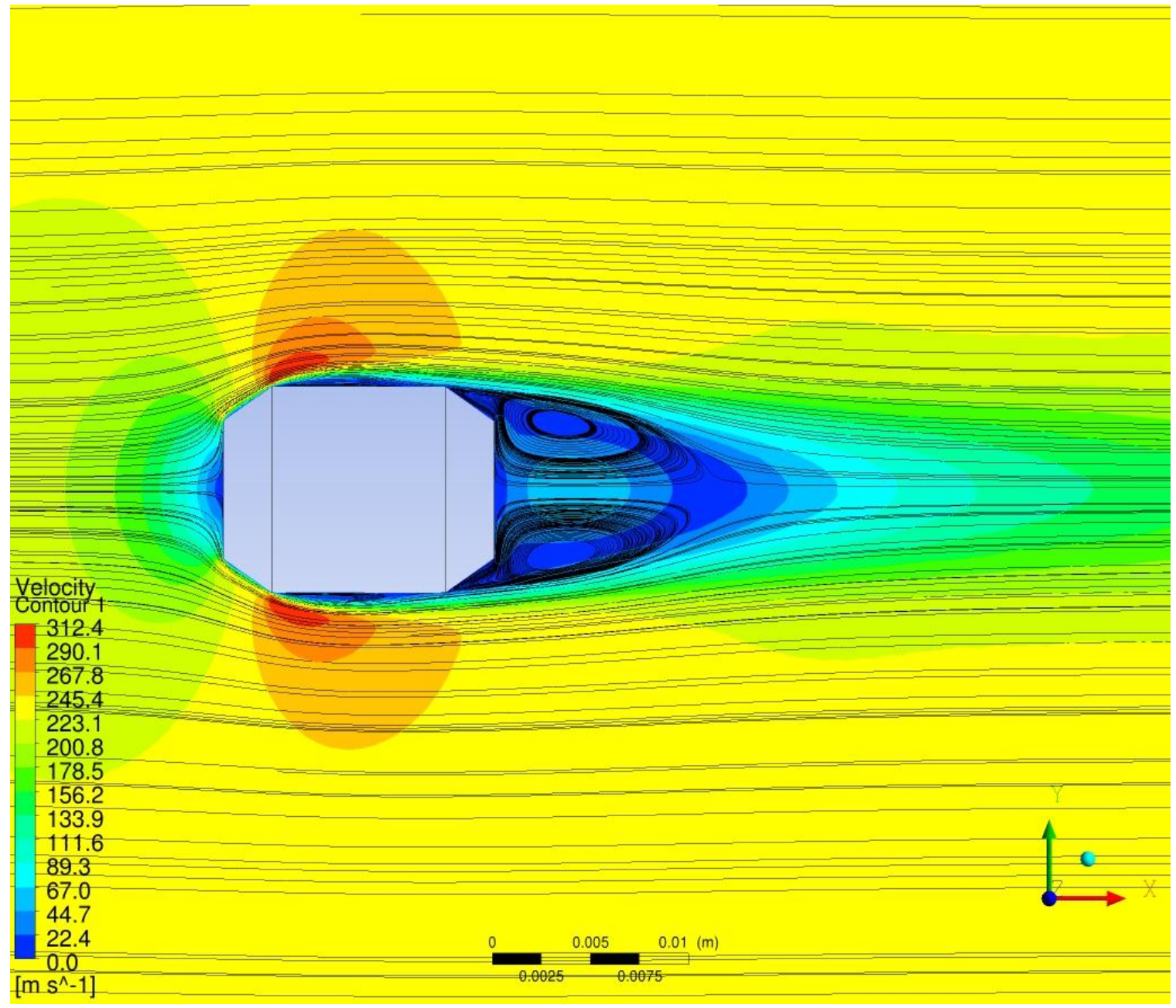

Figure 94. Velocity Contour with Streamlines of 3D, Steady-State, Spinning Target in Drift Region at a Reynolds Number of 3,600

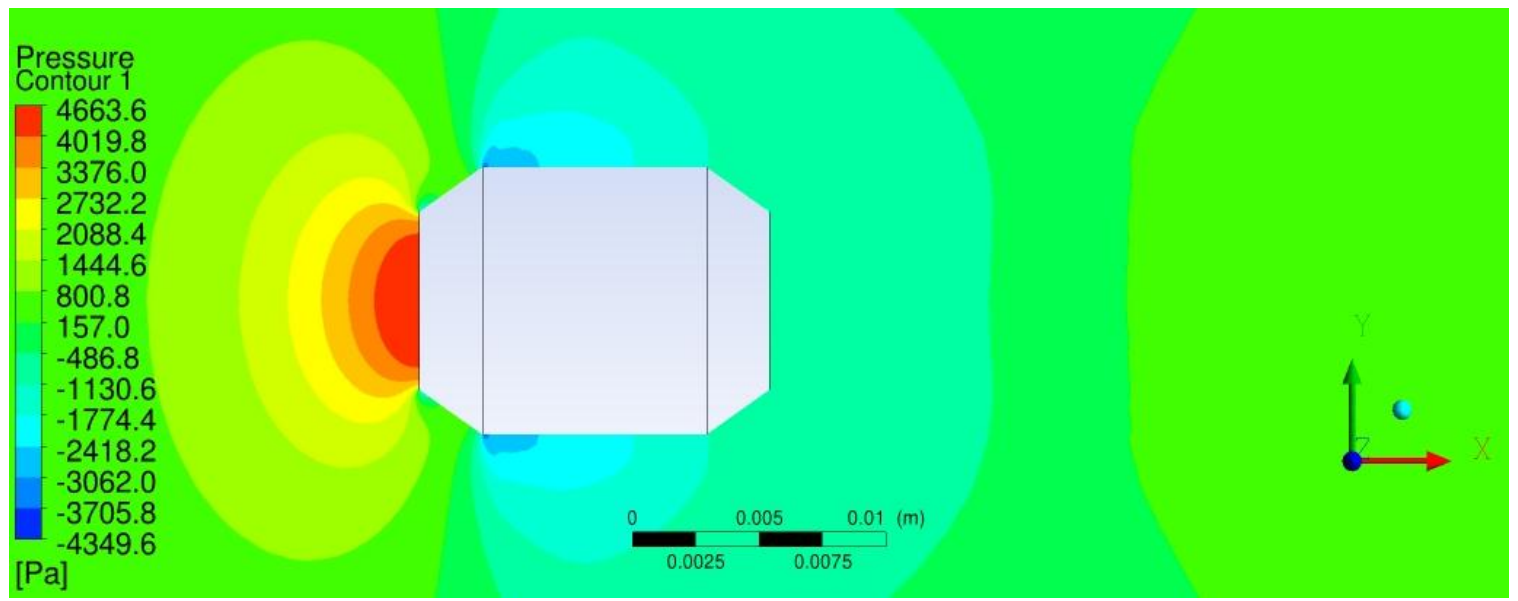

Figure 95. Pressure Contour of 3D, Steady-State, Spinning Target in Drift Region at a Reynolds Number of 4,400 


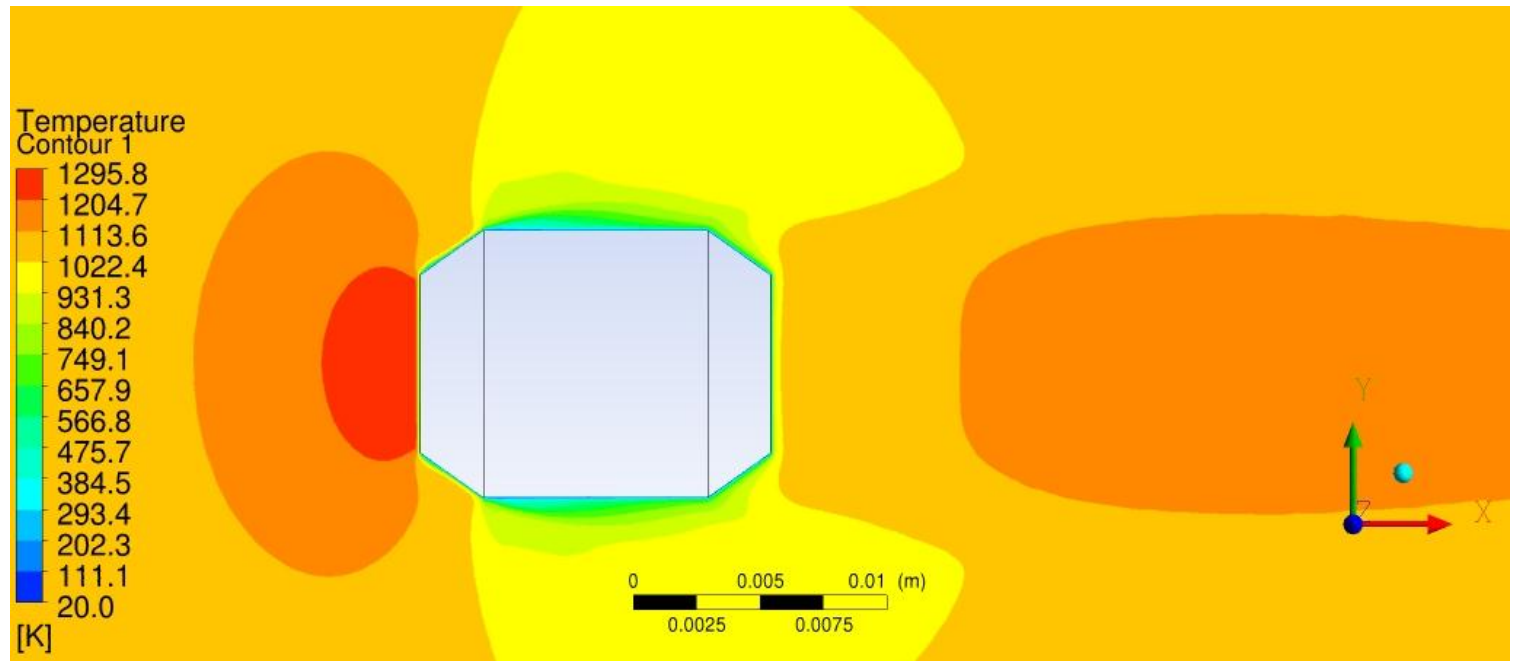

Figure 96. Temperature Contour of 3D, Steady-State, Spinning Target in Drift Region at a Reynolds Number of 4,400

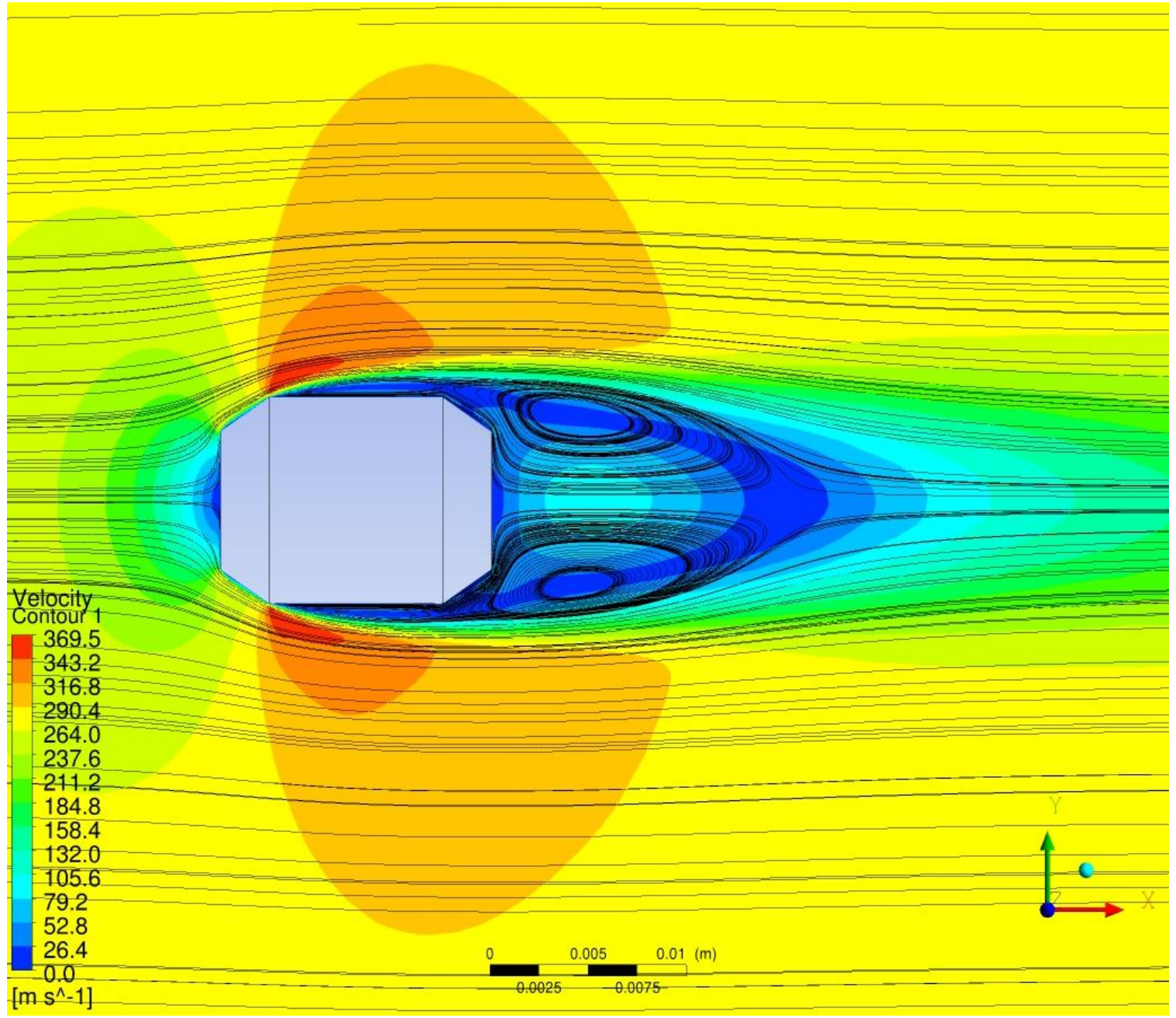

Figure 97. Velocity Contour with Streamlines of 3D, Steady-State, Spinning Target in Drift Region at a Reynolds Number of $\mathbf{4 , 4 0 0}$ 


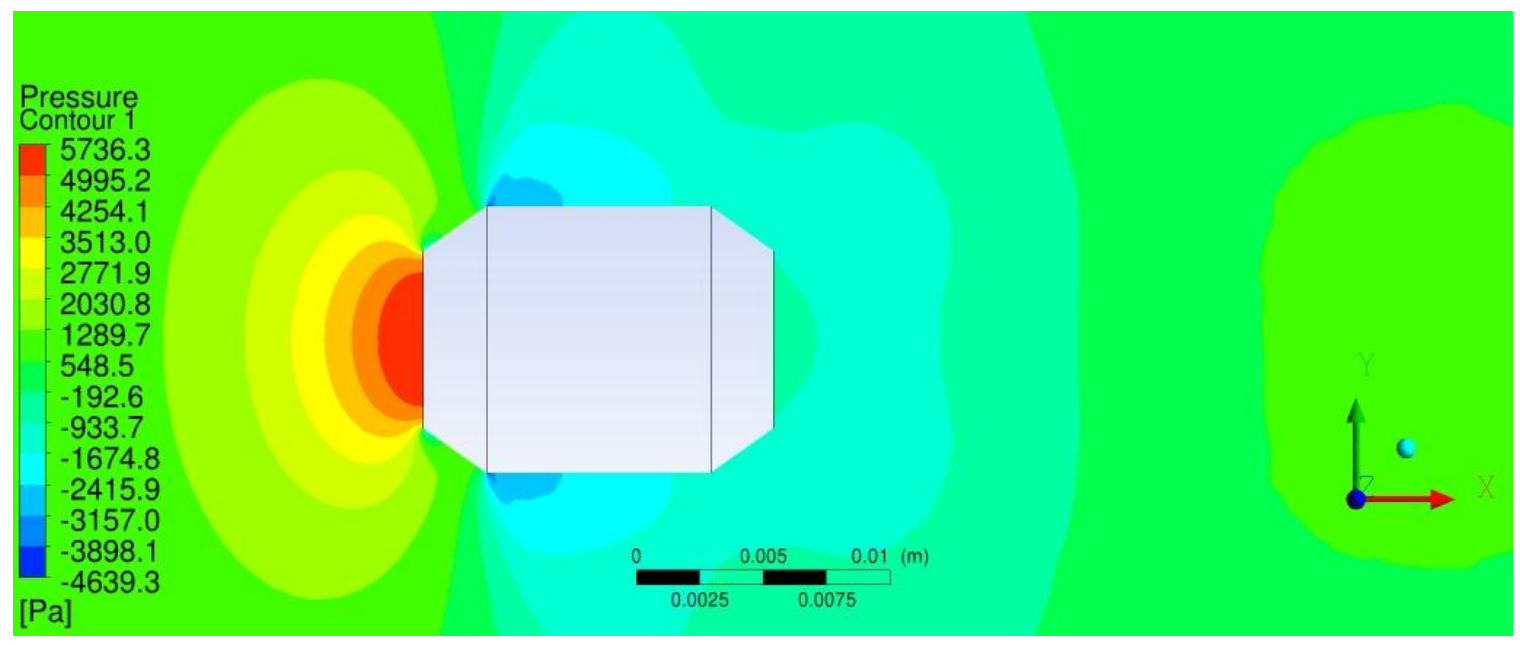

Figure 98. Pressure Contour of 3D, Steady-State, Spinning Target in Drift Region at a Reynolds Number of 4,800

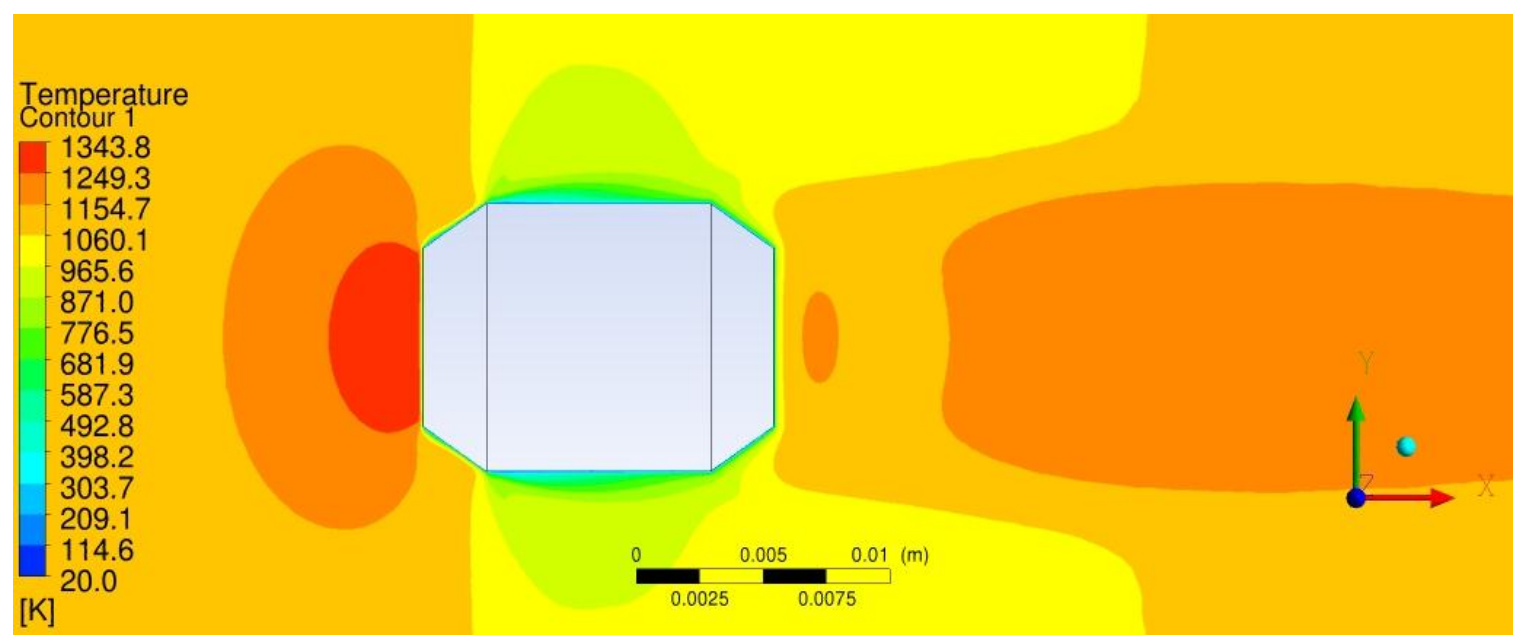

Figure 99. Temperature Contour of 3D, Steady-State, Spinning Target in Drift Region at a Reynolds Number of 4,800 


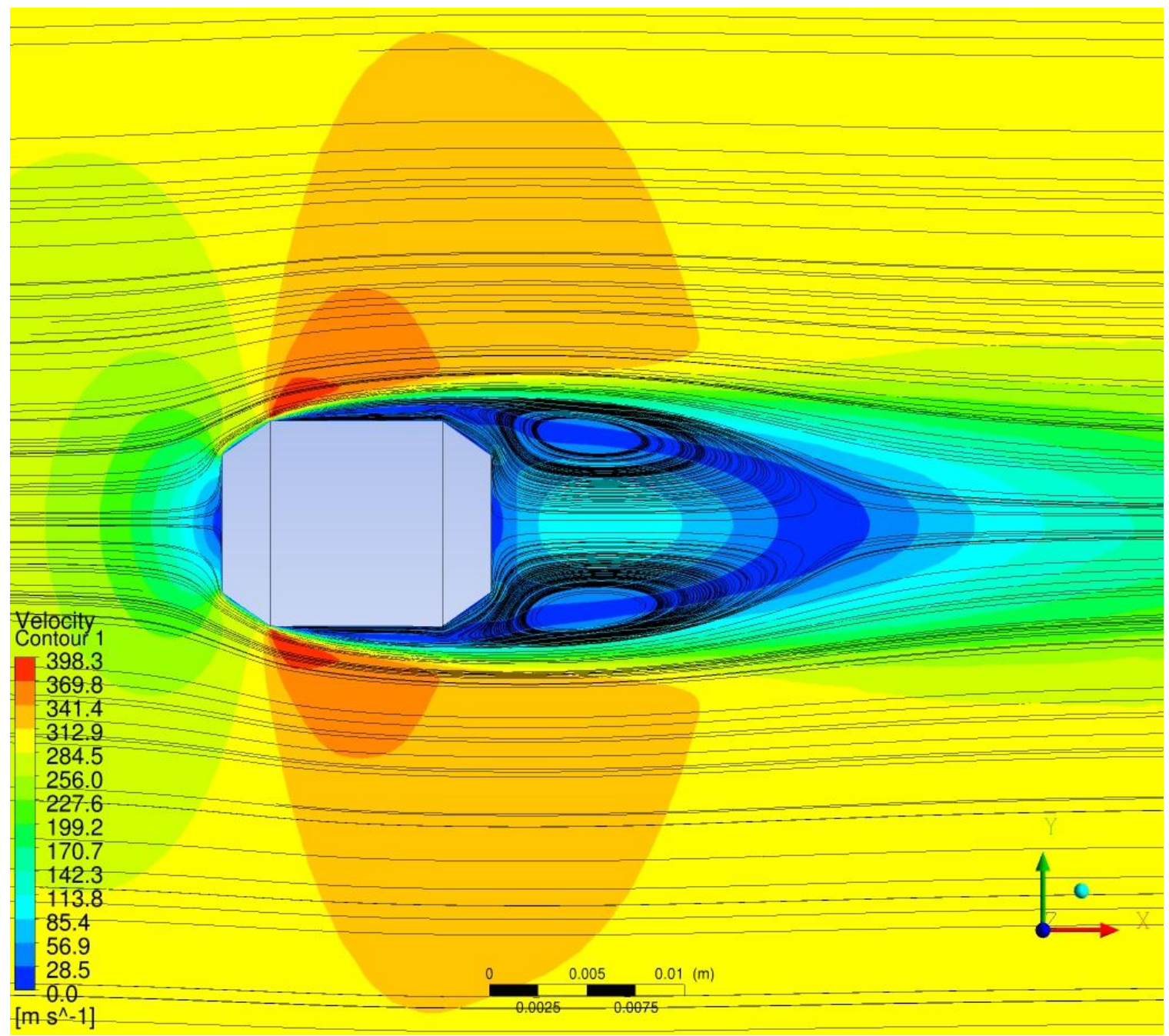

Figure 100. Velocity Contour with Streamlines of 3D, Steady-State, Spinning Target in Drift Region at a Reynolds Number of 4,800

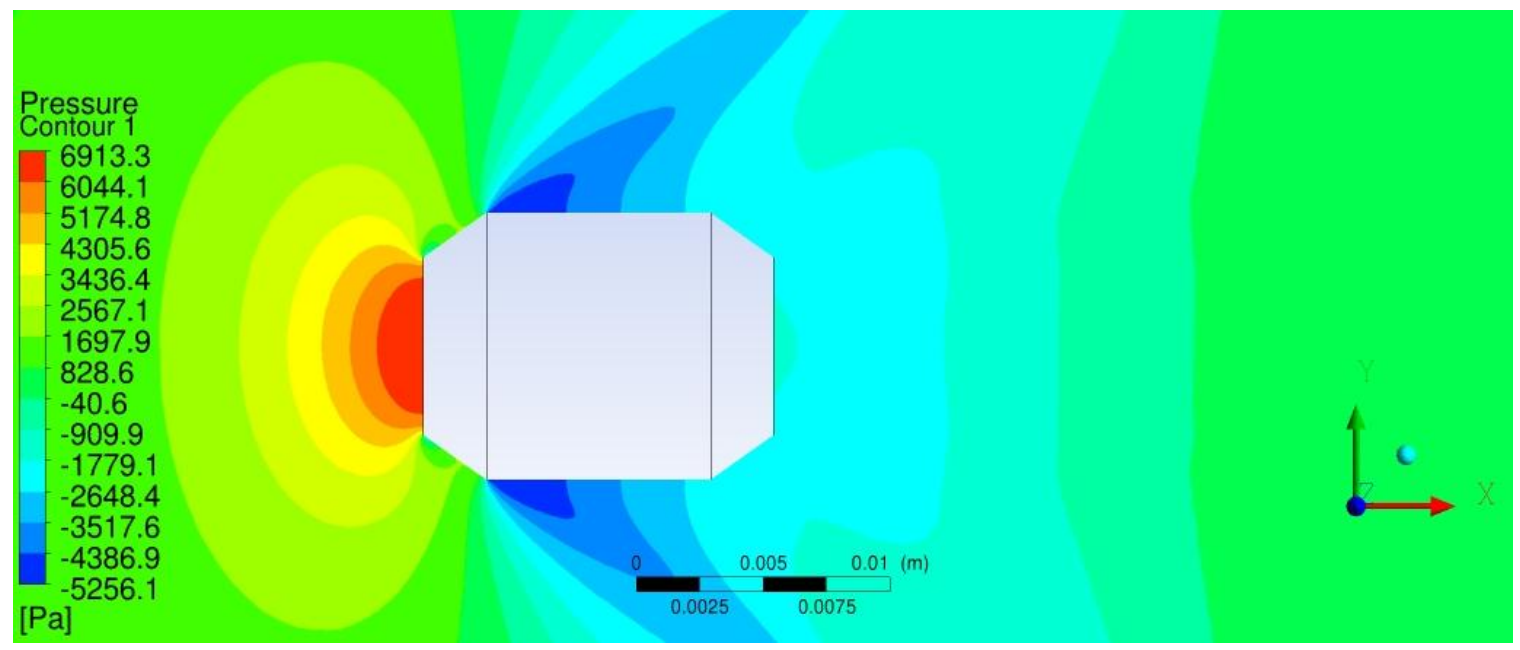

Figure 101. Pressure Contour of 3D, Steady-State, Spinning Target in Drift Region at a Reynolds Number of 5,200 


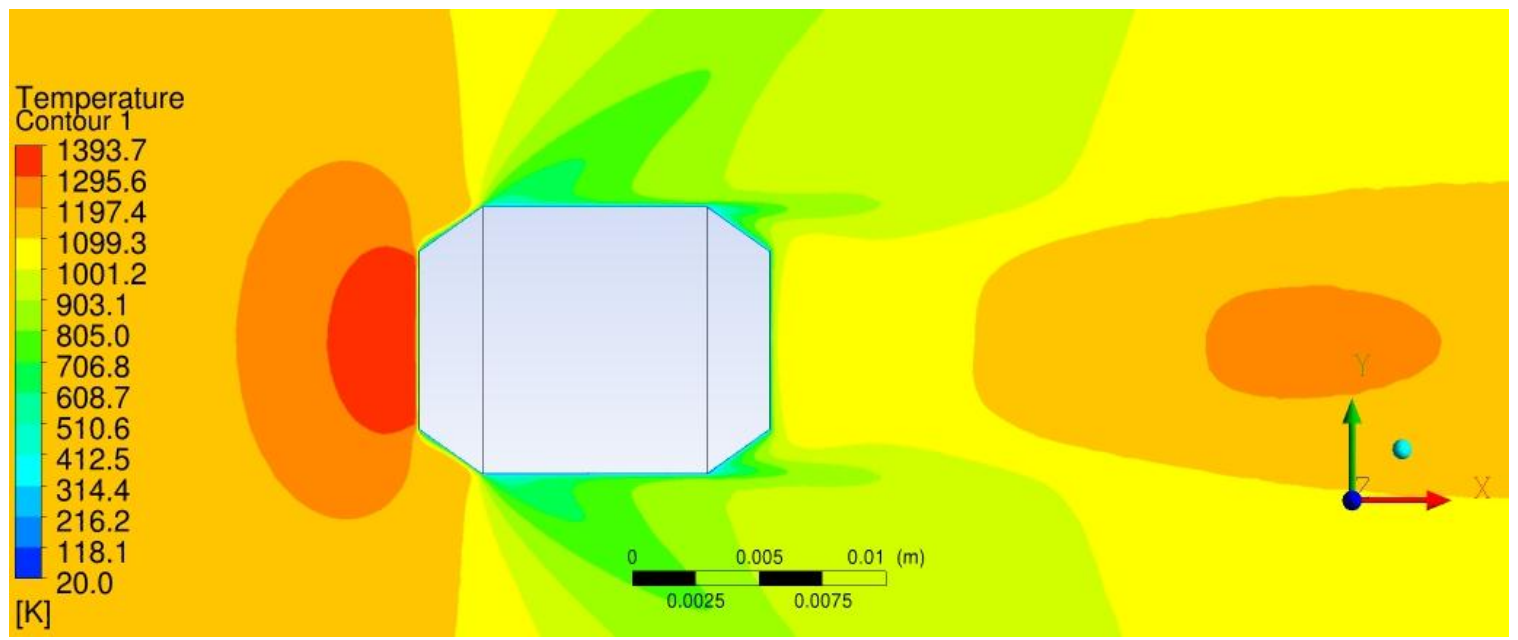

Figure 102. Temperature Contour of 3D, Steady-State, Spinning Target in Drift Region at a Reynolds Number of 5,200

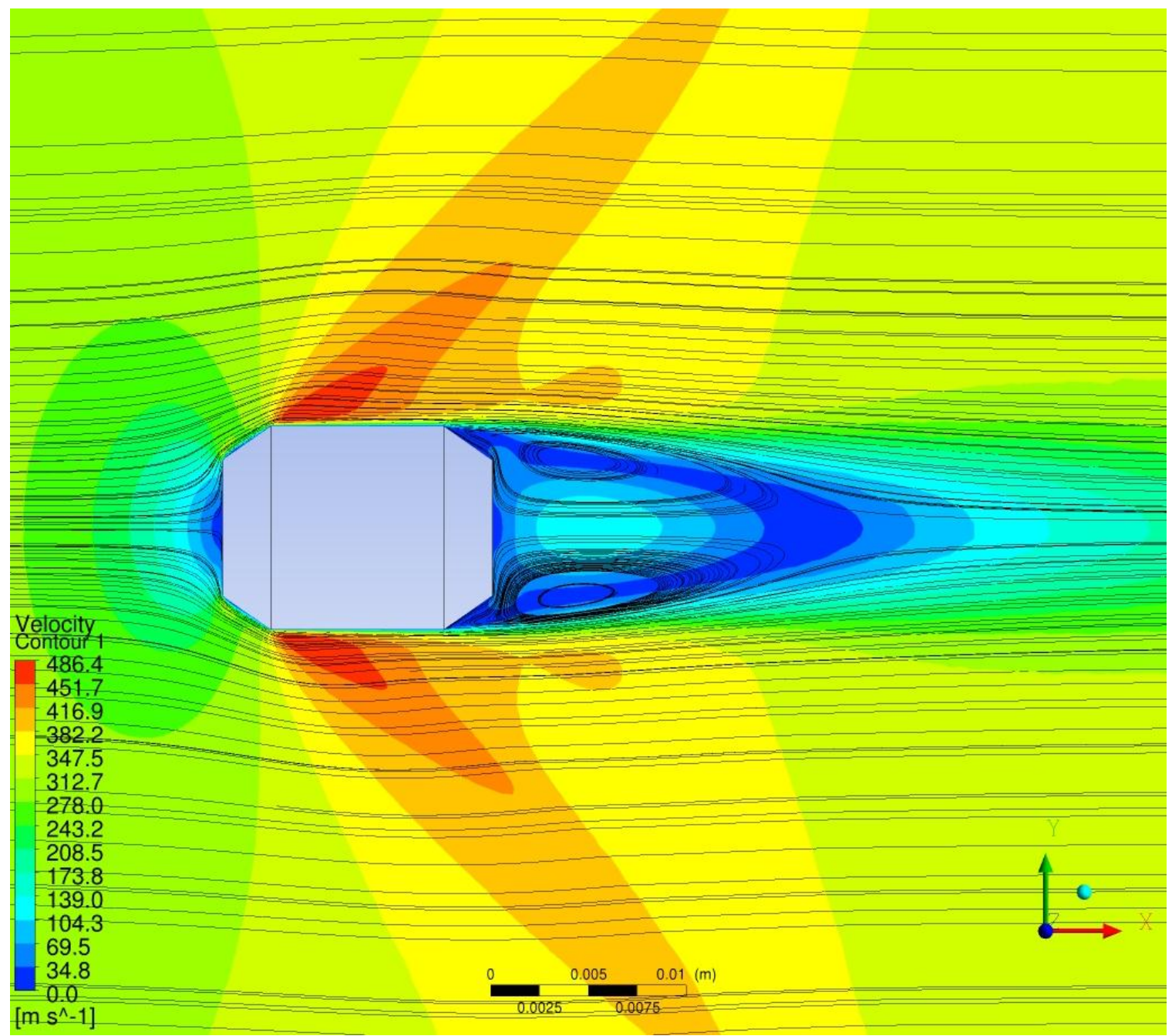

Figure 103. Velocity Contour with Streamlines of 3D, Steady-State, Spinning Target in Drift Region at a Reynolds Number of 5,200 


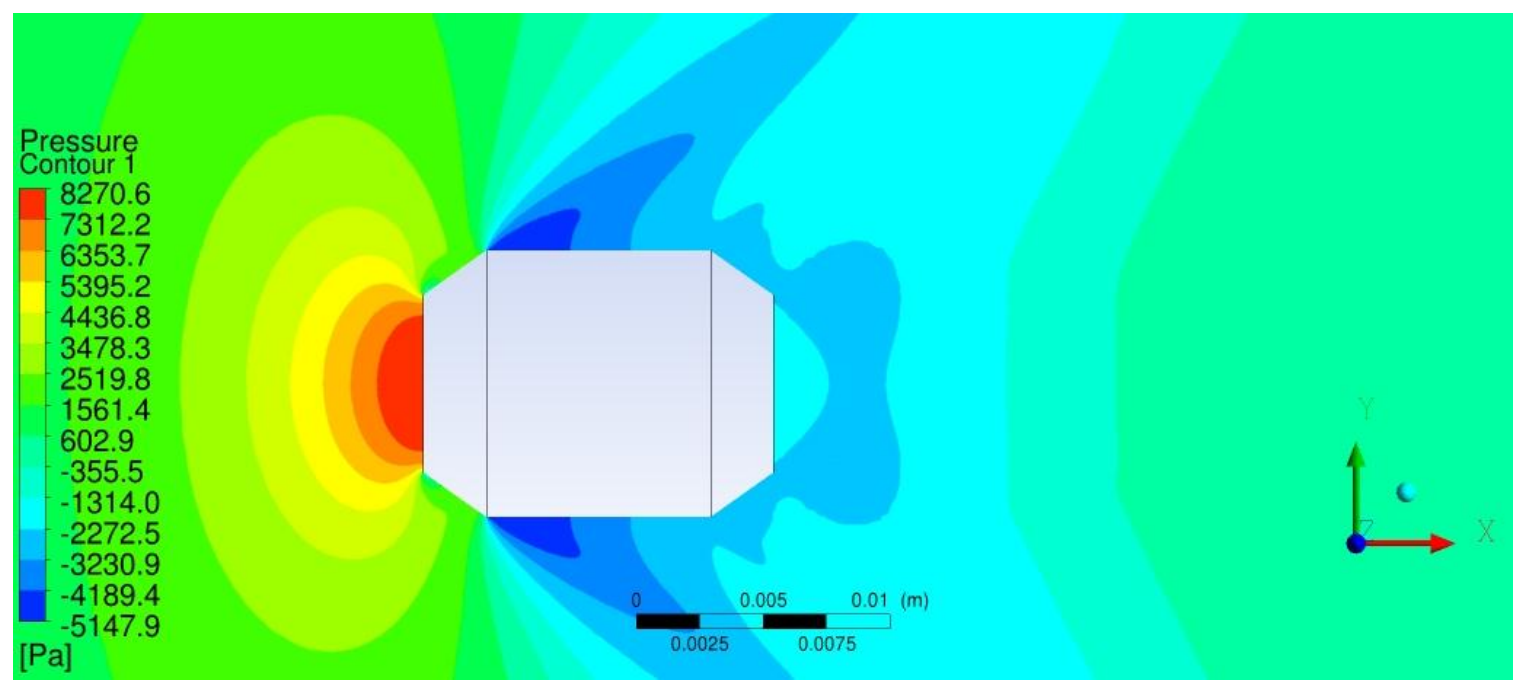

Figure 104. Pressure Contour of 3D, Steady-State, Spinning Target in Drift Region at a Reynolds Number of 5,600

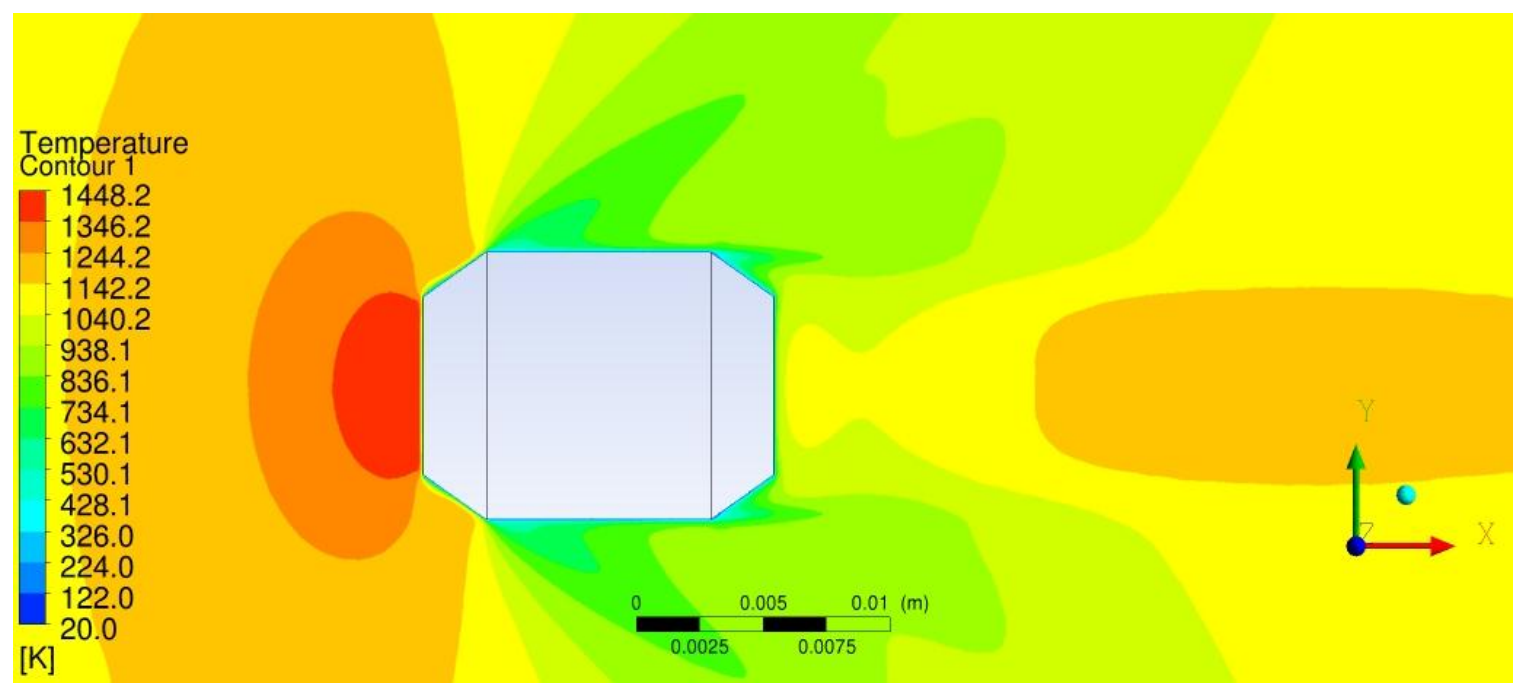

Figure 105. Temperature Contour of 3D, Steady-State, Spinning Target in Drift Region at a Reynolds Number of $\mathbf{5 , 6 0 0}$ 


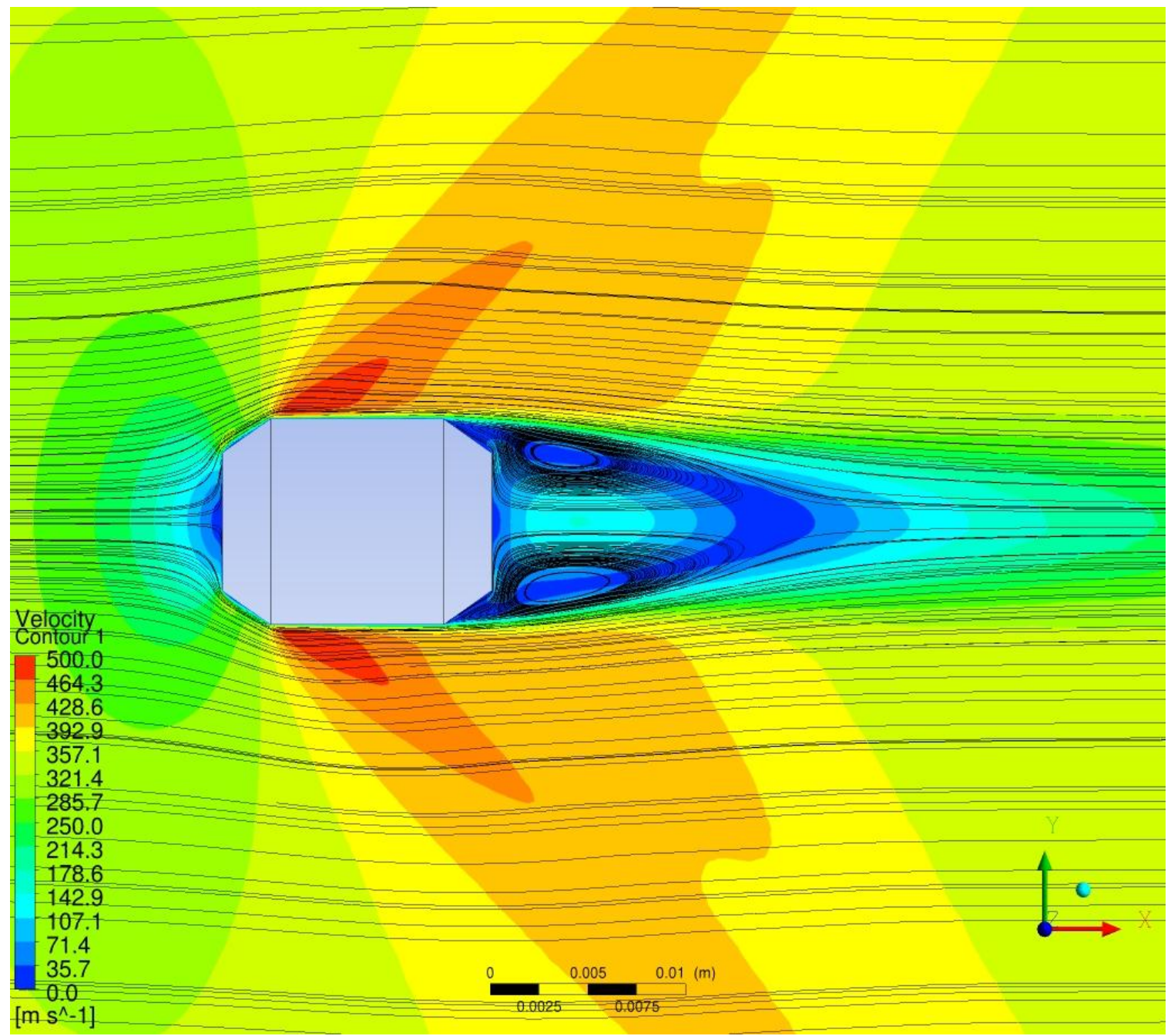

Figure 106. Velocity Contour with Streamlines of 3D, Steady-State, Spinning Target in Drift Region at a Reynolds Number of 5,600

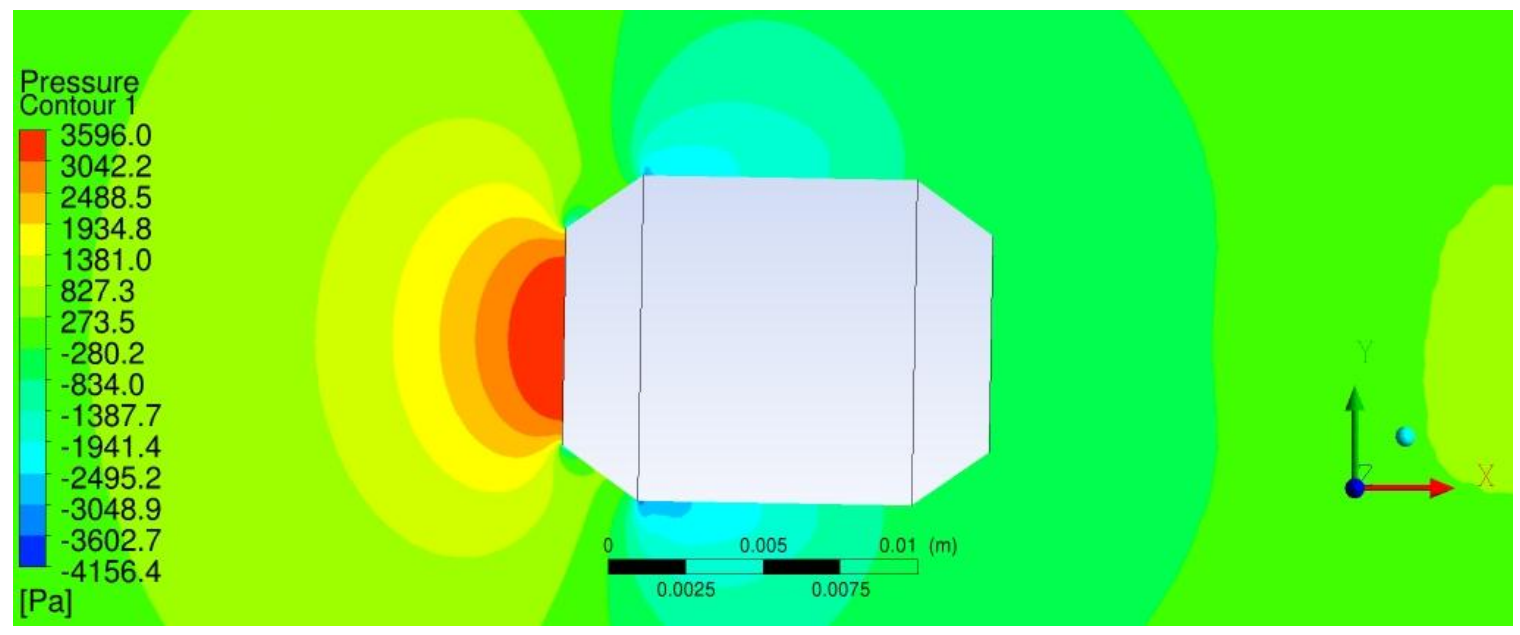

Figure 107. Pressure Contour of 3D, Steady-State, Spinning Target in Drift Region with a $1^{\circ}$ Angle of Attack 


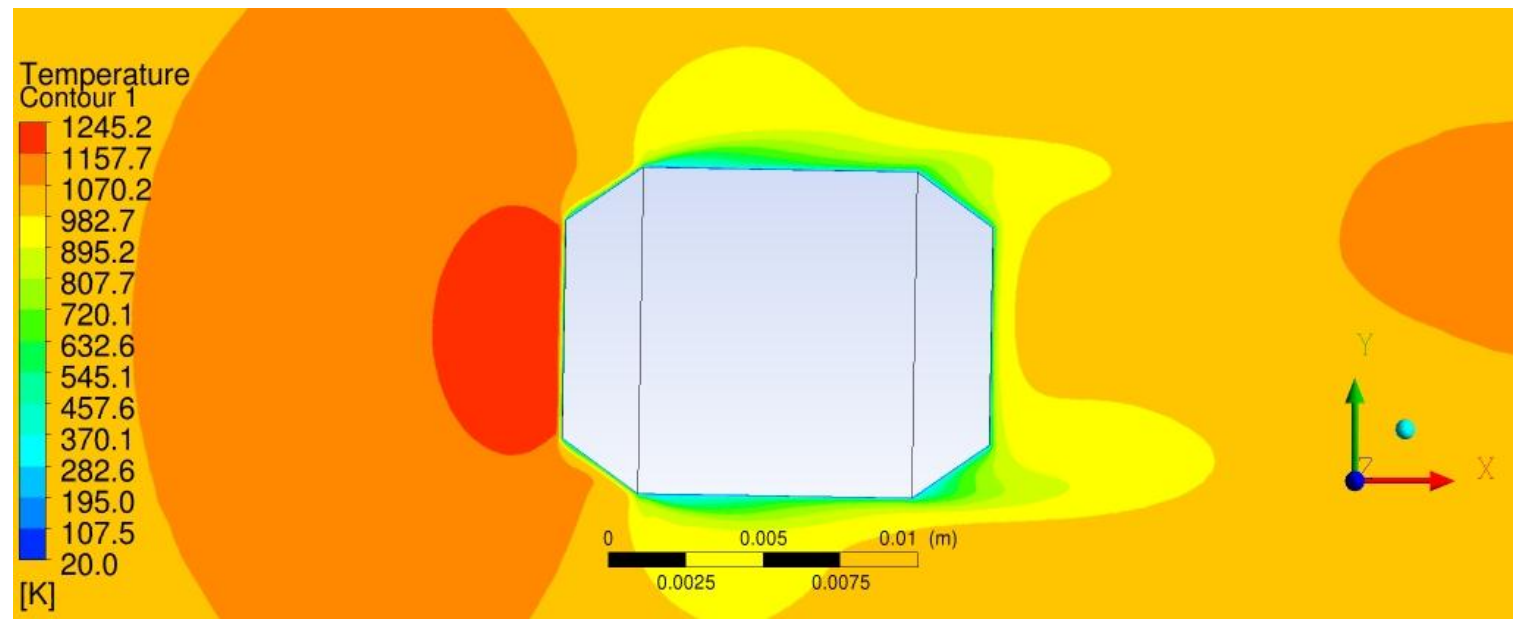

Figure 108. Temperature Contour of 3D, Steady-State, Spinning Target in Drift Region with a $1^{\circ}$ Angle of Attack

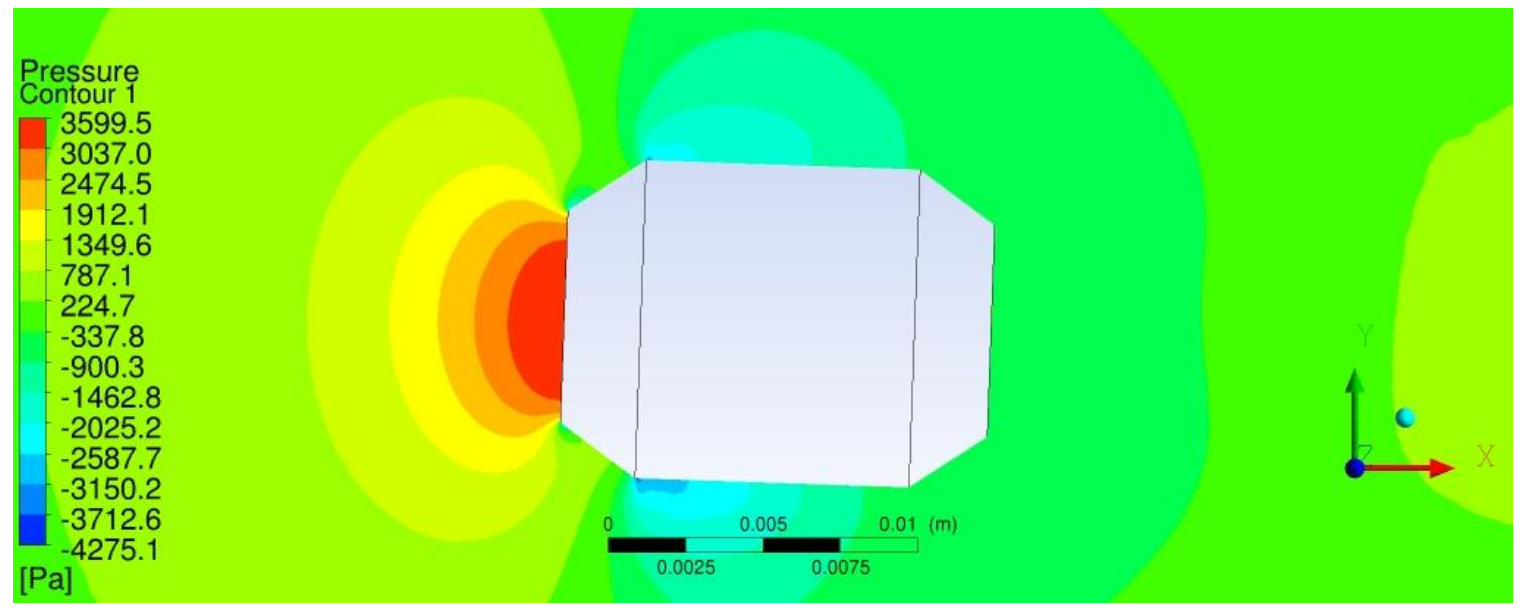

Figure 109. Pressure Contour of 3D, Steady-State, Spinning Target in Drift Region with a $2^{\circ}$ Angle of Attack

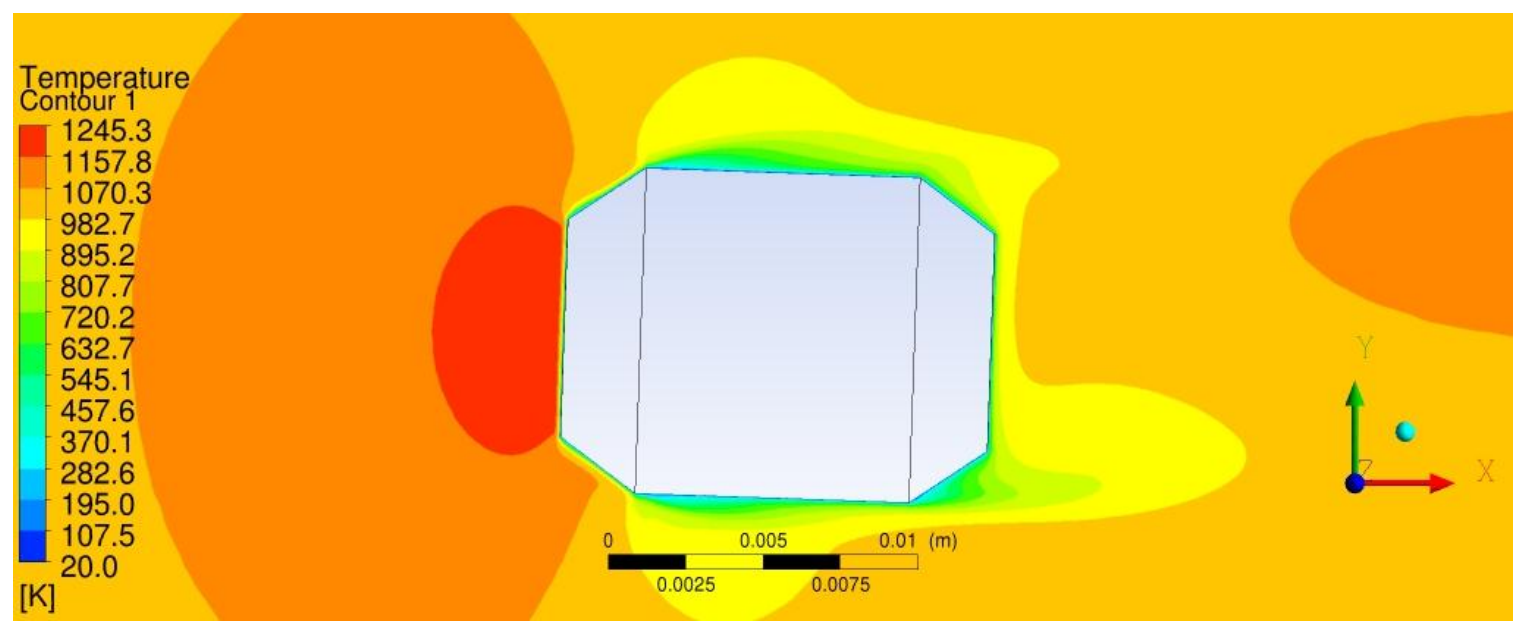

Figure 110. Temperature Contour of 3D, Steady-State, Spinning Target in Drift Region with a $2^{\circ}$ Angle of Attack 


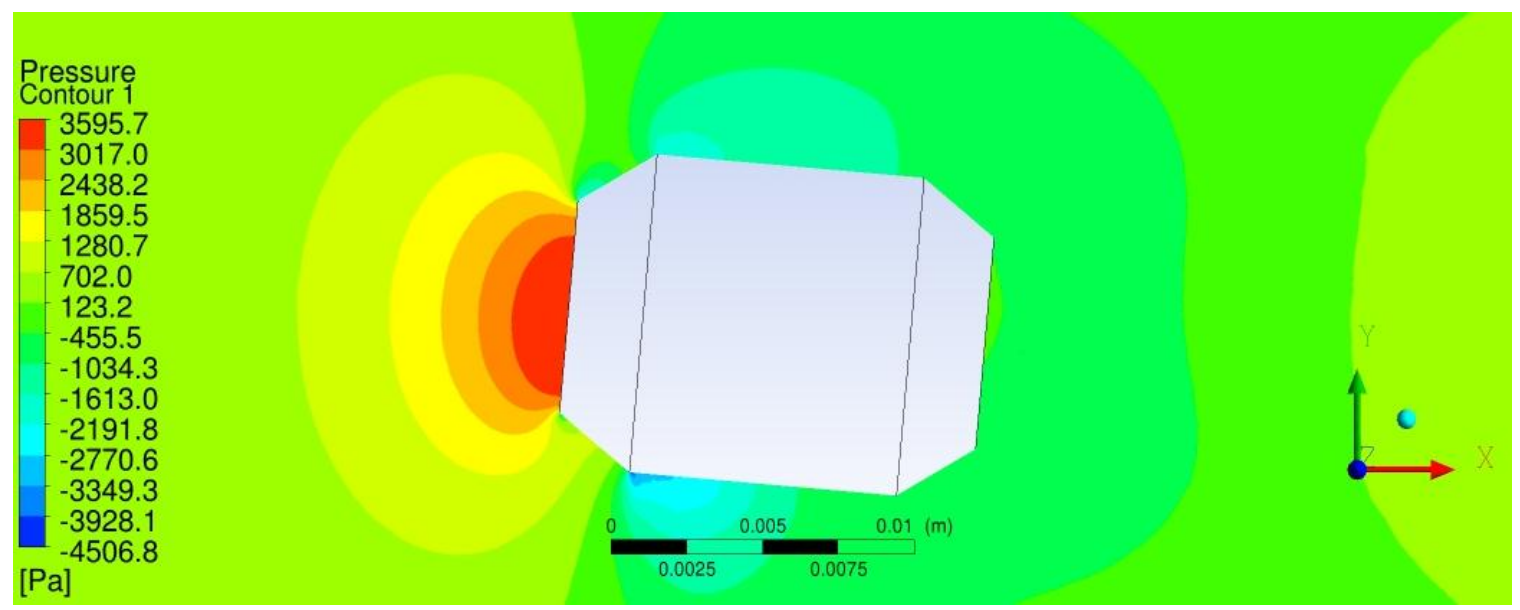

Figure 111. Pressure Contour of 3D, Steady-State, Spinning Target in Drift Region with a $5^{\circ}$ Angle of Attack

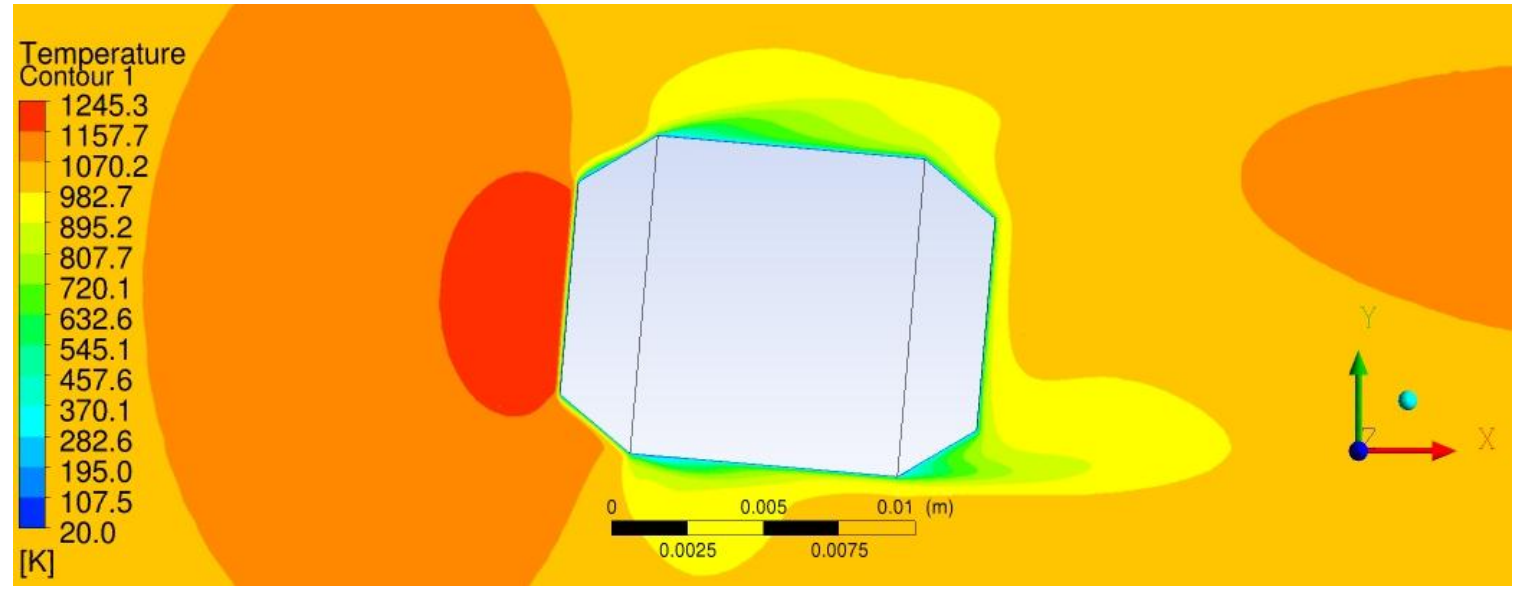

Figure 112. Temperature Contour of 3D, Steady-State, Spinning Target in Drift Region with a $5^{\circ}$ Angle of Attack

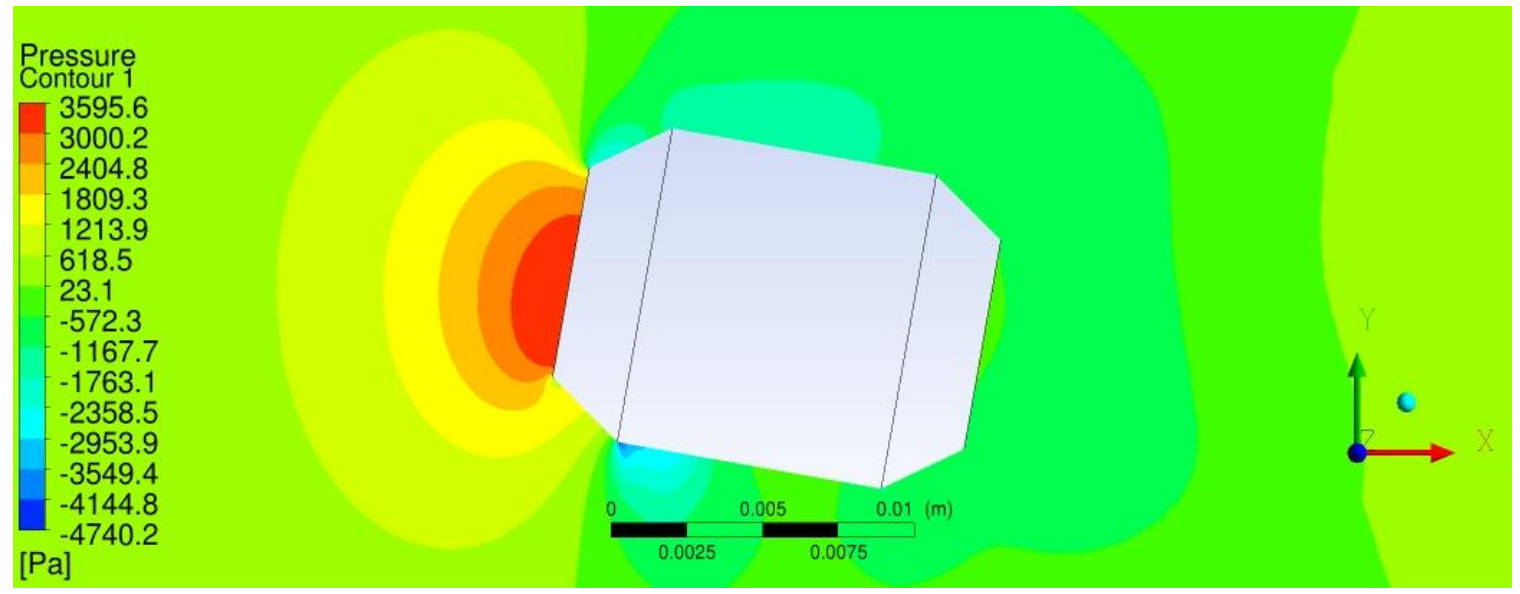

Figure 113. Pressure Contour of 3D, Steady-State, Spinning Target in Drift Region with a $10^{\circ}$ Angle of Attack 


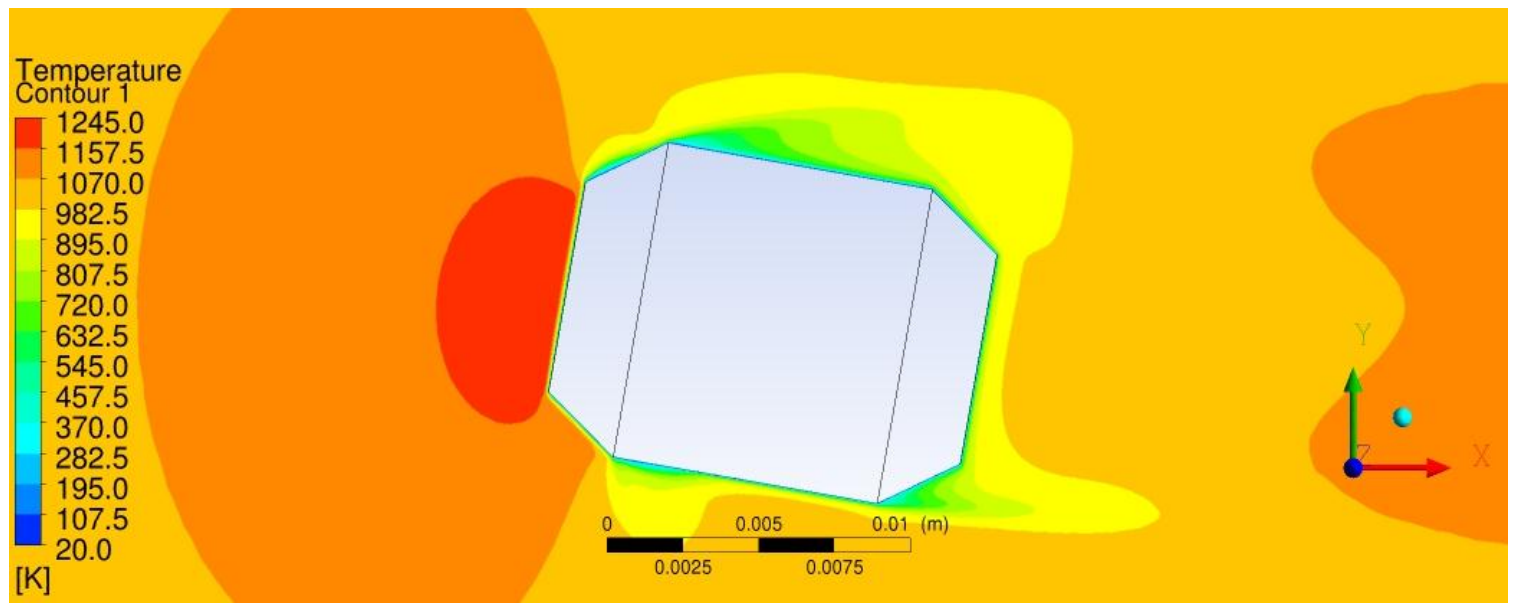

Figure 114. Temperature Contour of 3D, Steady-State, Spinning Target in Drift Region with a $10^{\circ}$ Angle of Attack

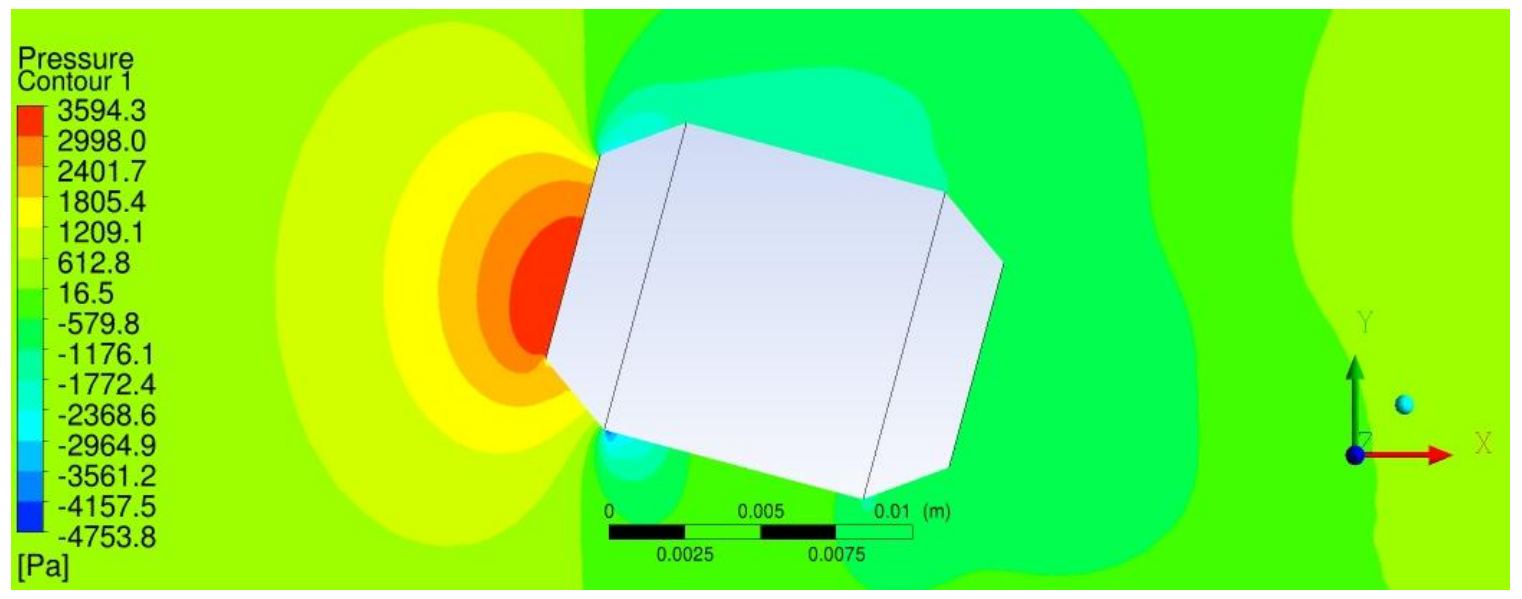

Figure 115. Pressure Contour of 3D, Steady-State, Spinning Target in Drift Region with a $15^{\circ}$ Angle of Attack

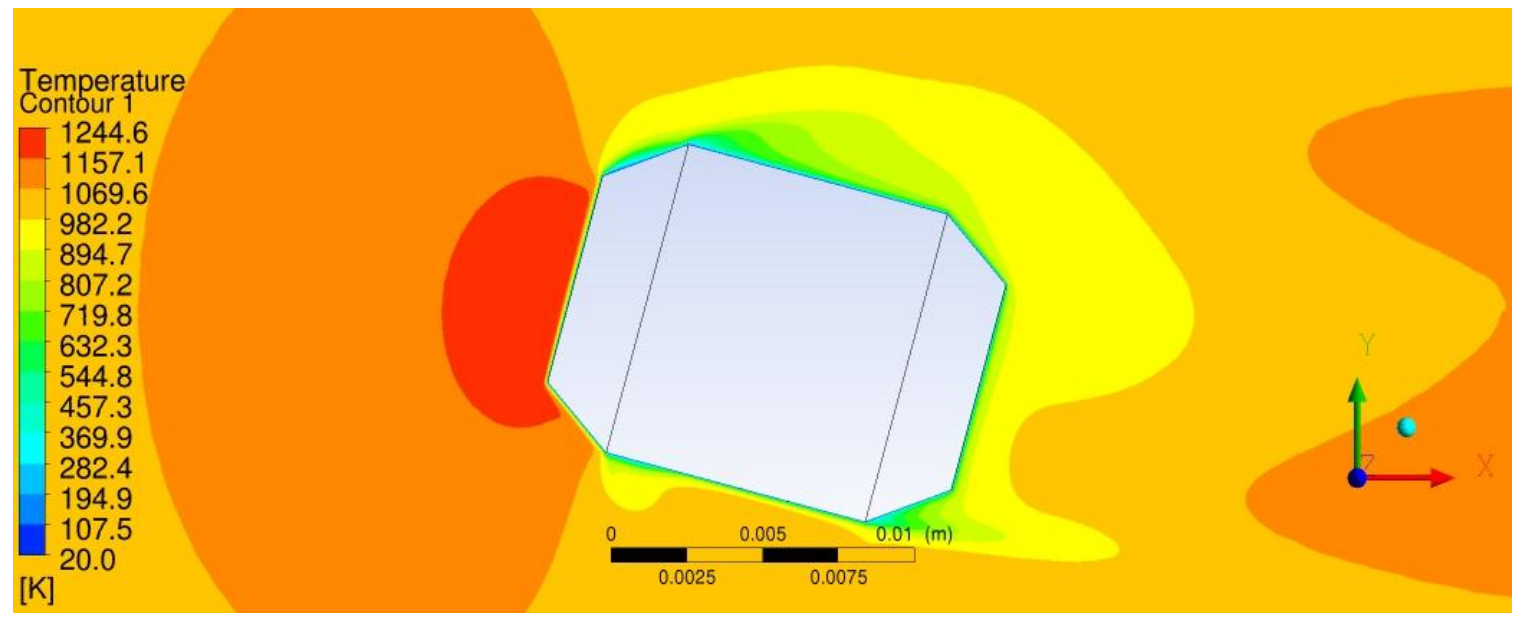

Figure 116. Temperature Contour of 3D, Steady-State, Spinning Target in Drift Region with a $15^{\circ}$ Angle of Attack 


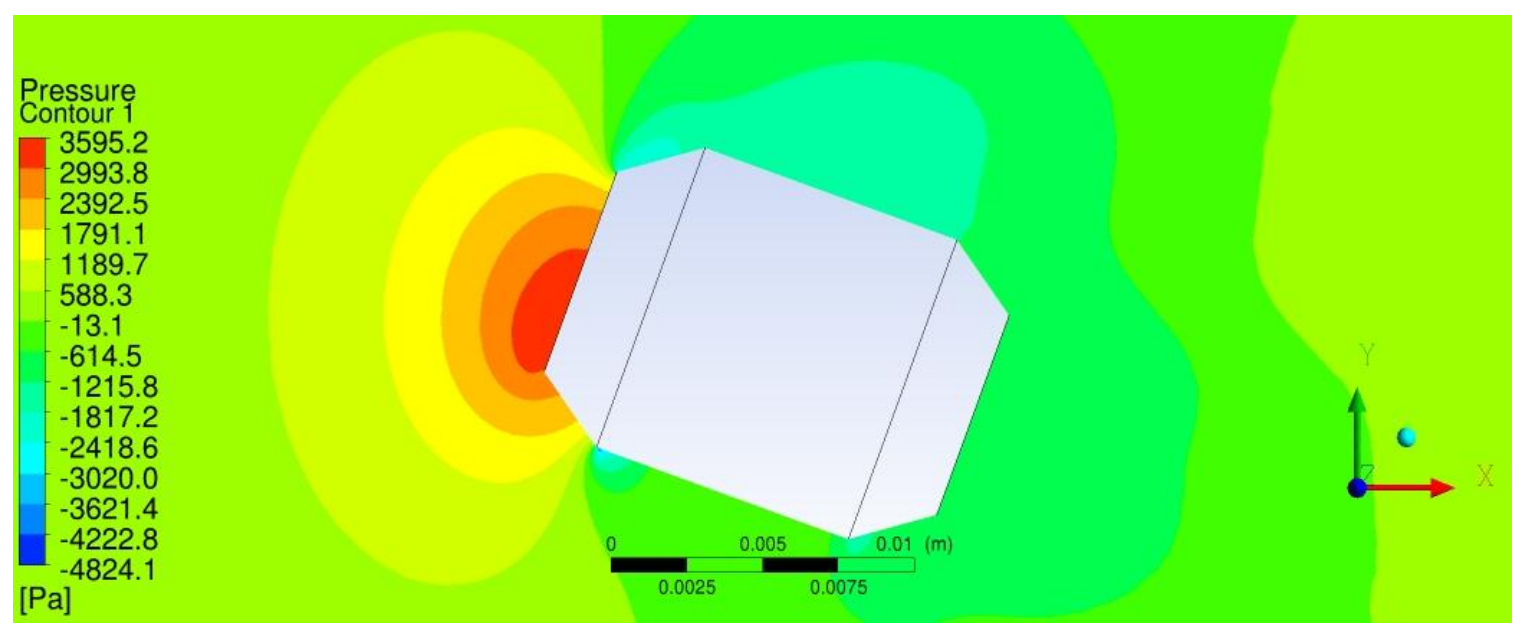

Figure 117. Pressure Contour of 3D, Steady-State, Spinning Target in Drift Region with a $20^{\circ}$ Angle of Attack

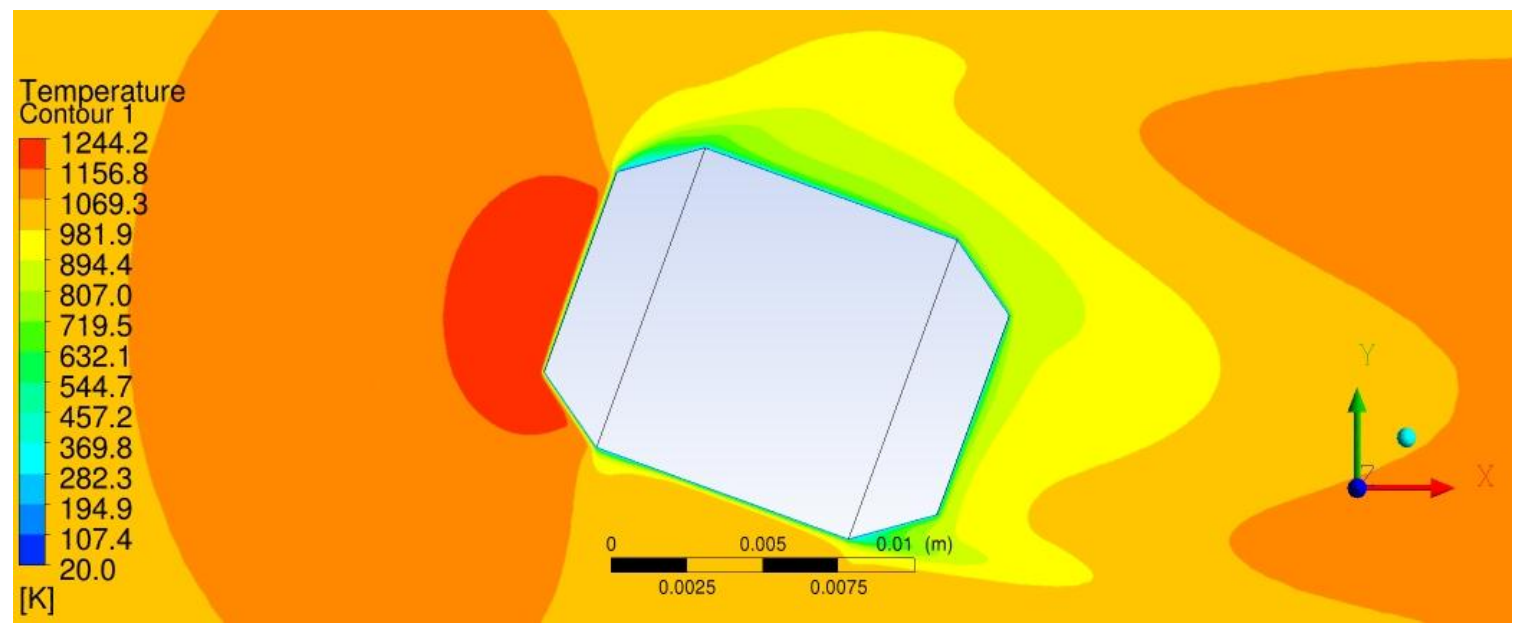

Figure 118. Temperature Contour of 3D, Steady-State, Spinning Target in Drift Region with a $20^{\circ}$ Angle of Attack 


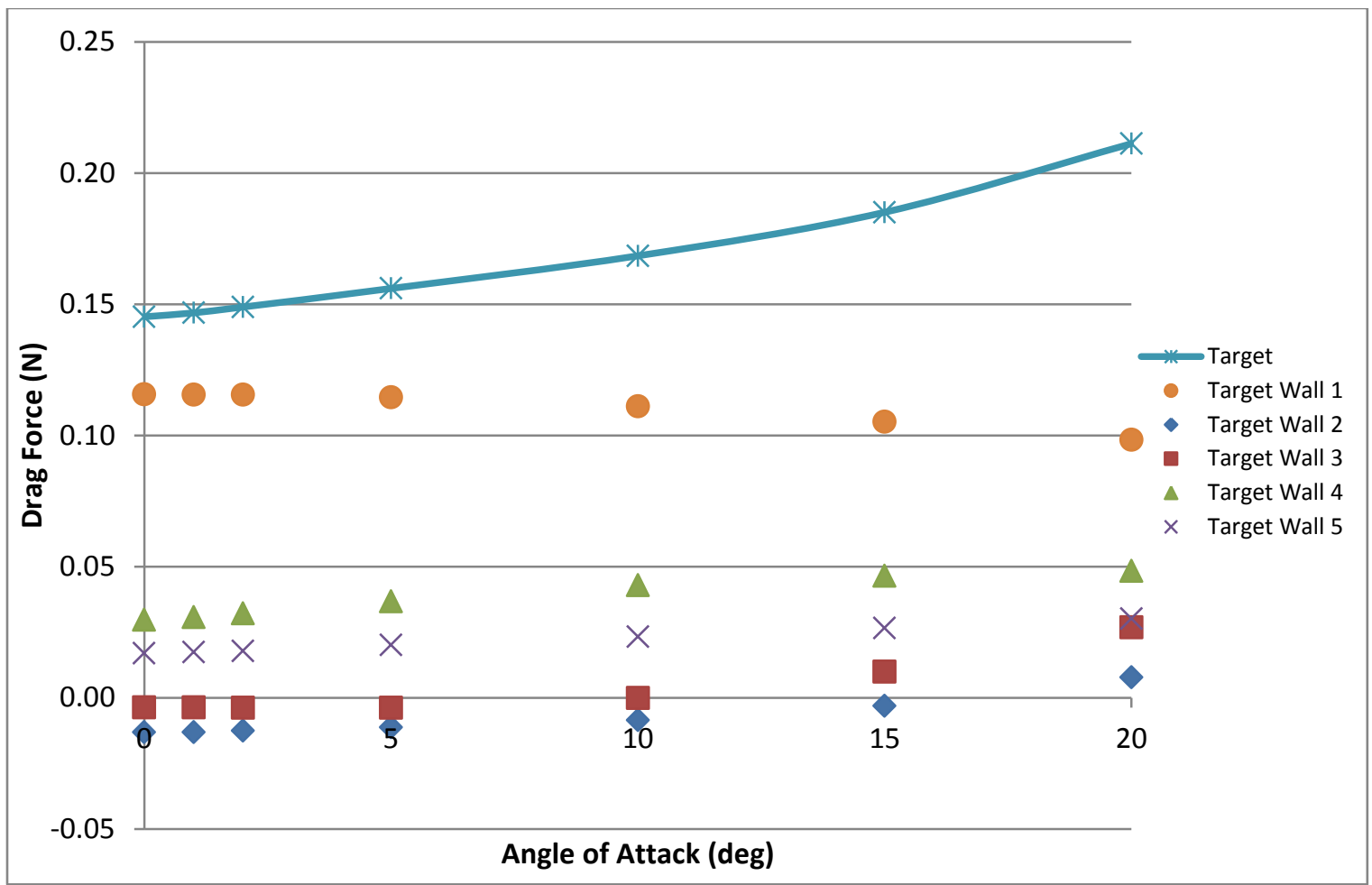

Figure 119. Drag Force on Spinning Target in Drift Region at Different Angles of Attack from 3D, Steady-State Model

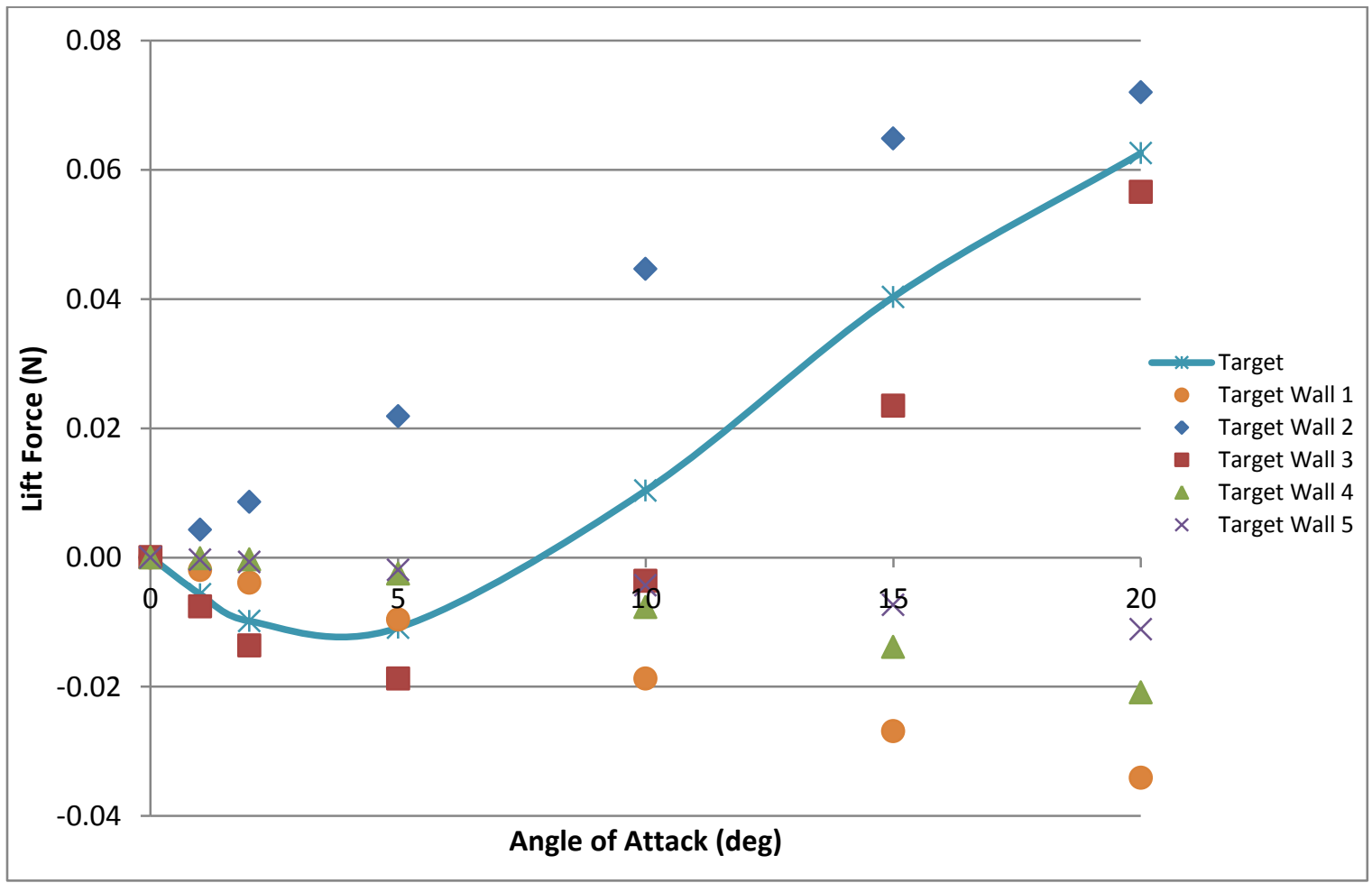

Figure 120. Lift Force on Spinning Target in Drift Region at Different Angles of Attack from 3D, Steady-State Model 


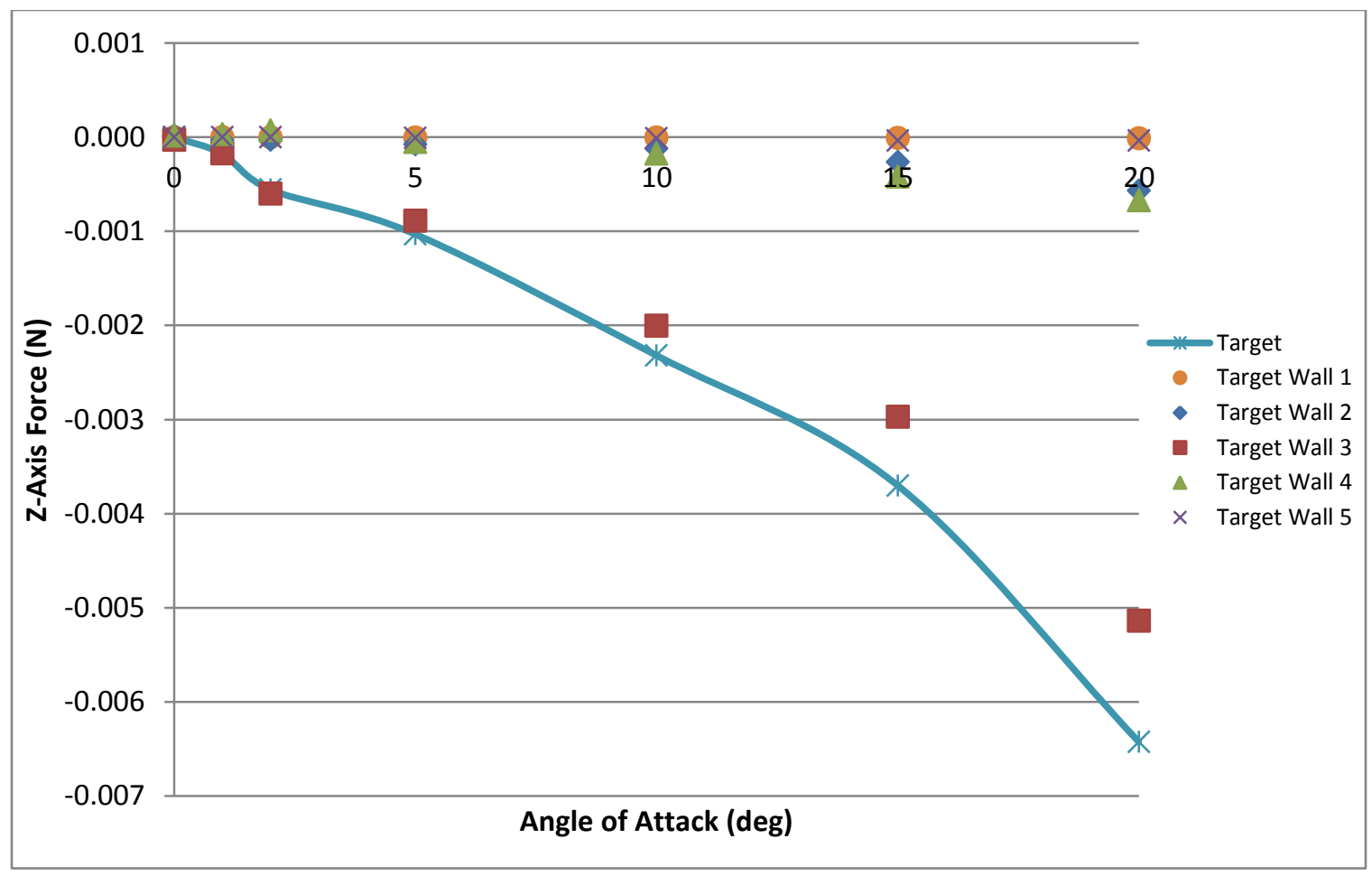

Figure 121. Z-Axis Force on Spinning Target in Drift Region at Different Angles of Attack from 3D, Steady-State Model

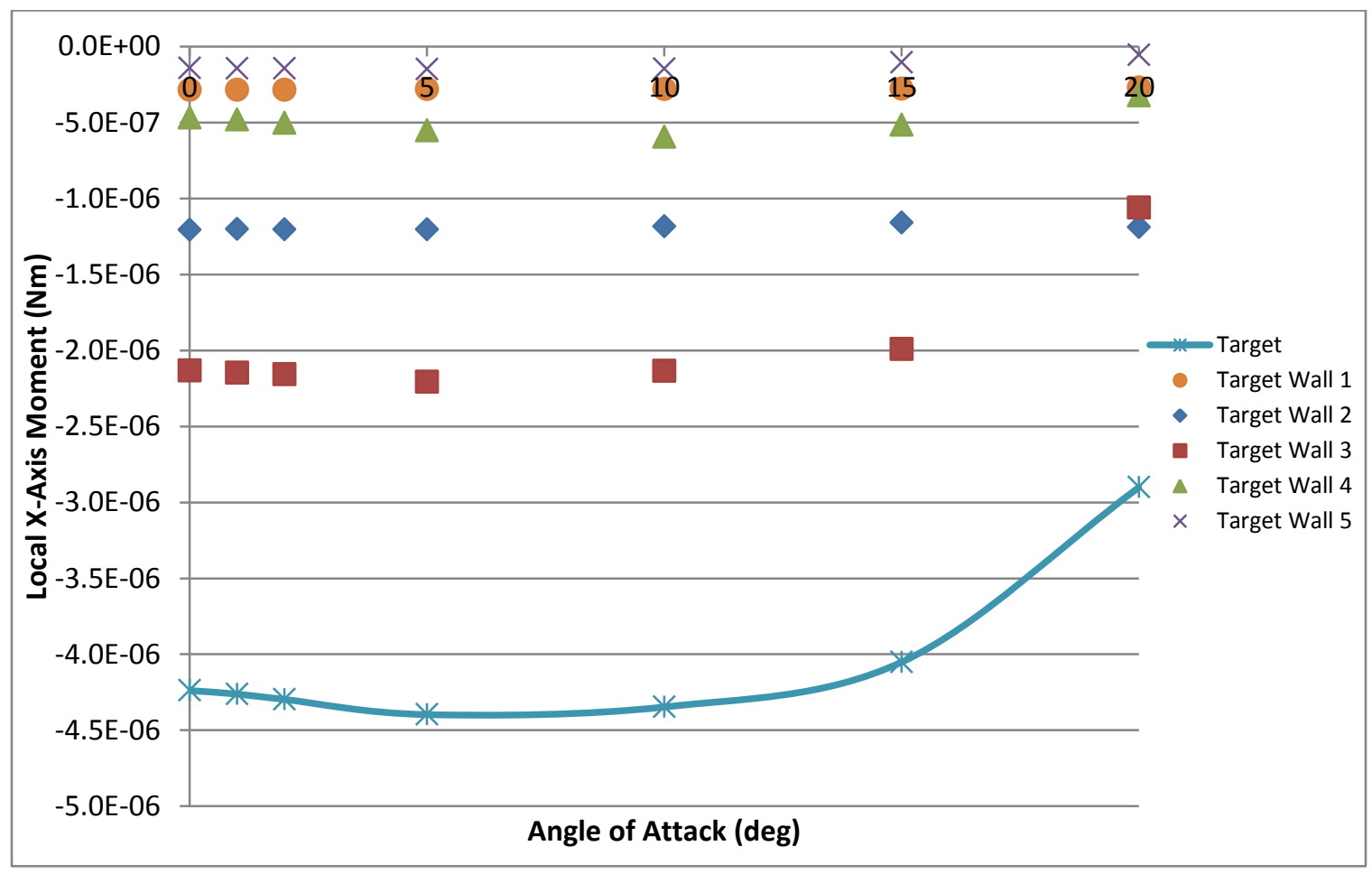

Figure 122. Local X-Axis (Axial) Moment on Spinning Target in Drift Region at Different Angles of Attack from 3D, Steady-State Model 


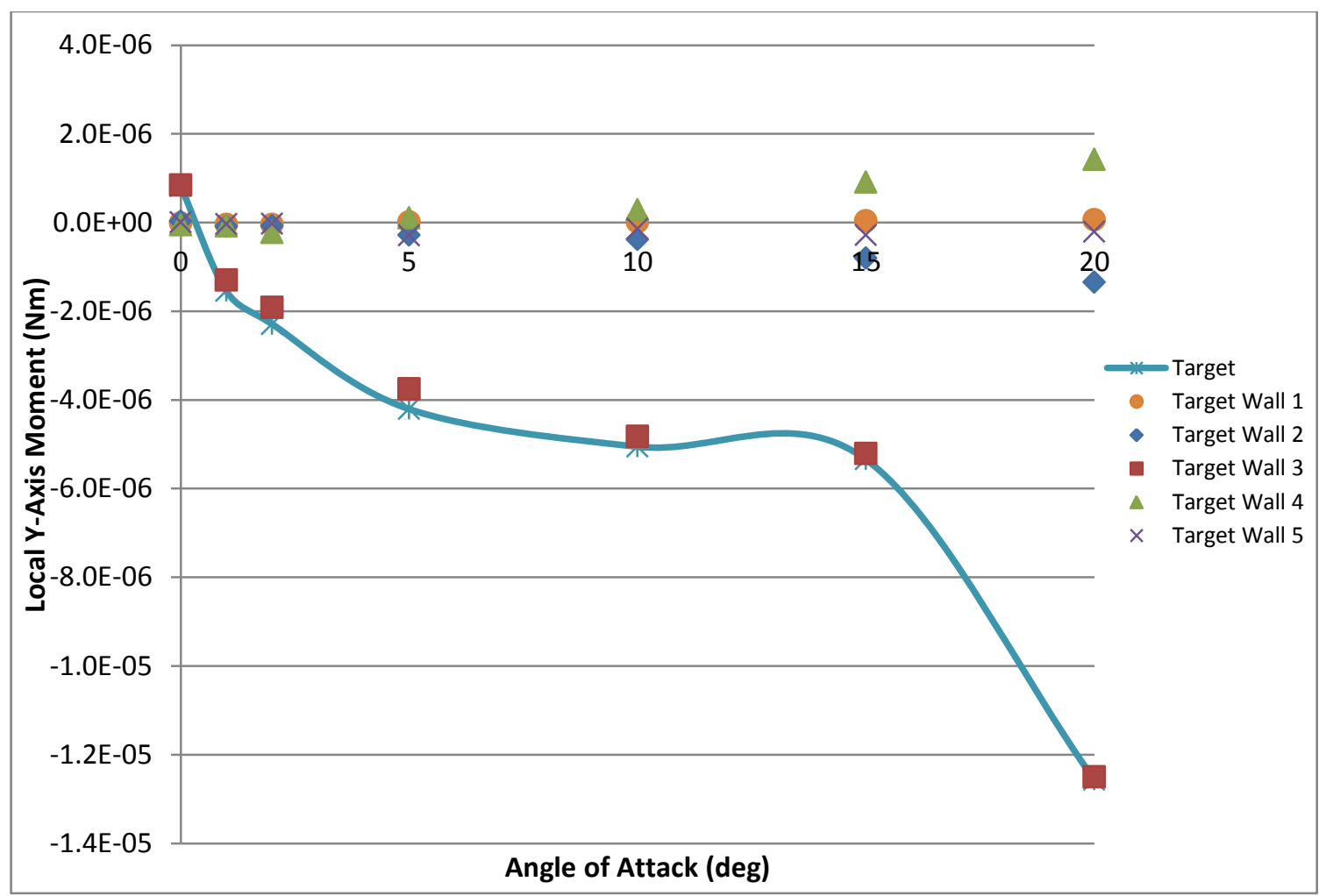

Figure 123. Local Y-Axis (Negative Magnus) Moment on Spinning Target in Drift Region at Different Angles of Attack from 3D, Steady-State Model

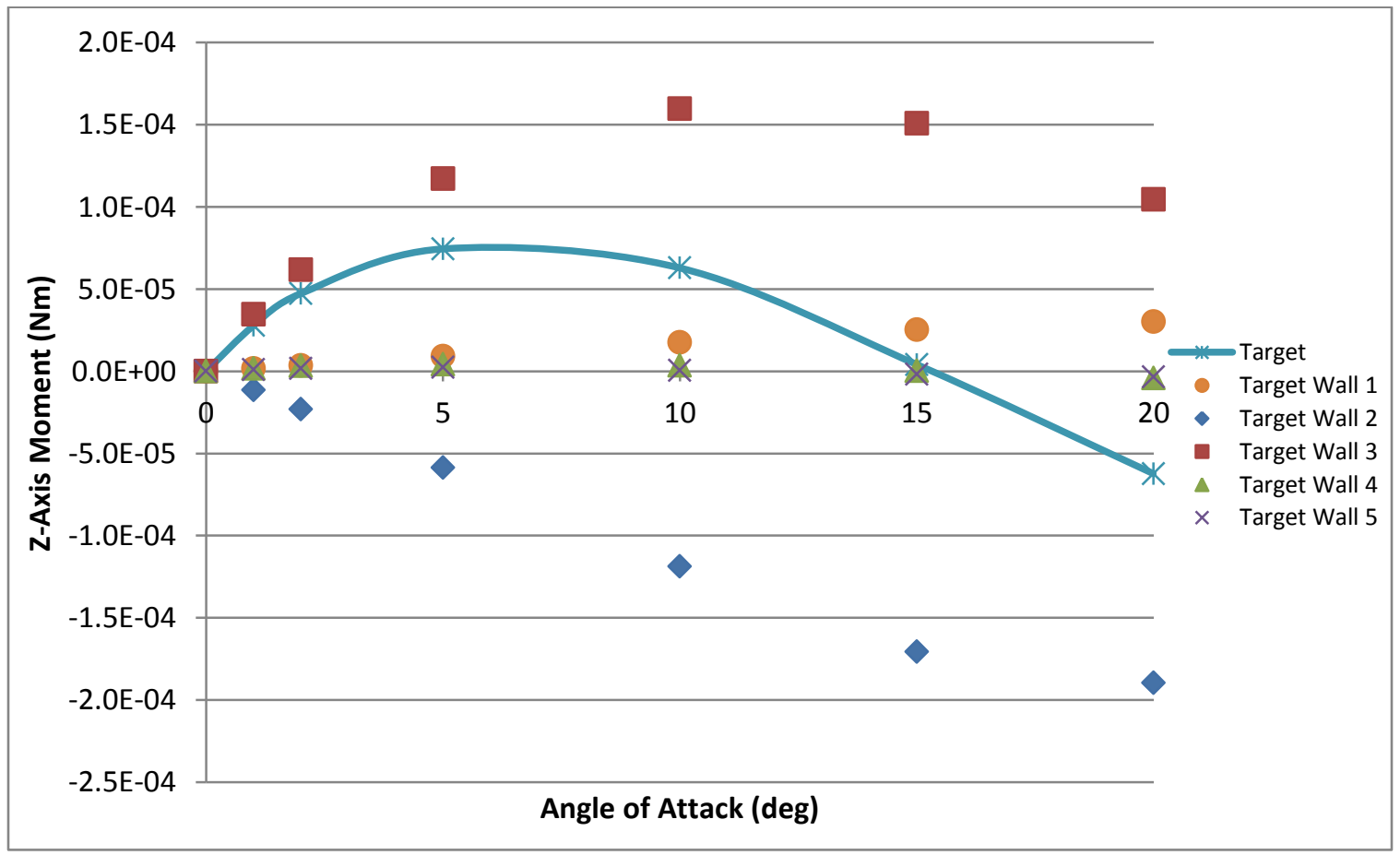

Figure 124. Z-Axis (Negative Overturning) Moment on Spinning Target in Drift Region at Different Angles of Attack from 3D, Steady-State Model 


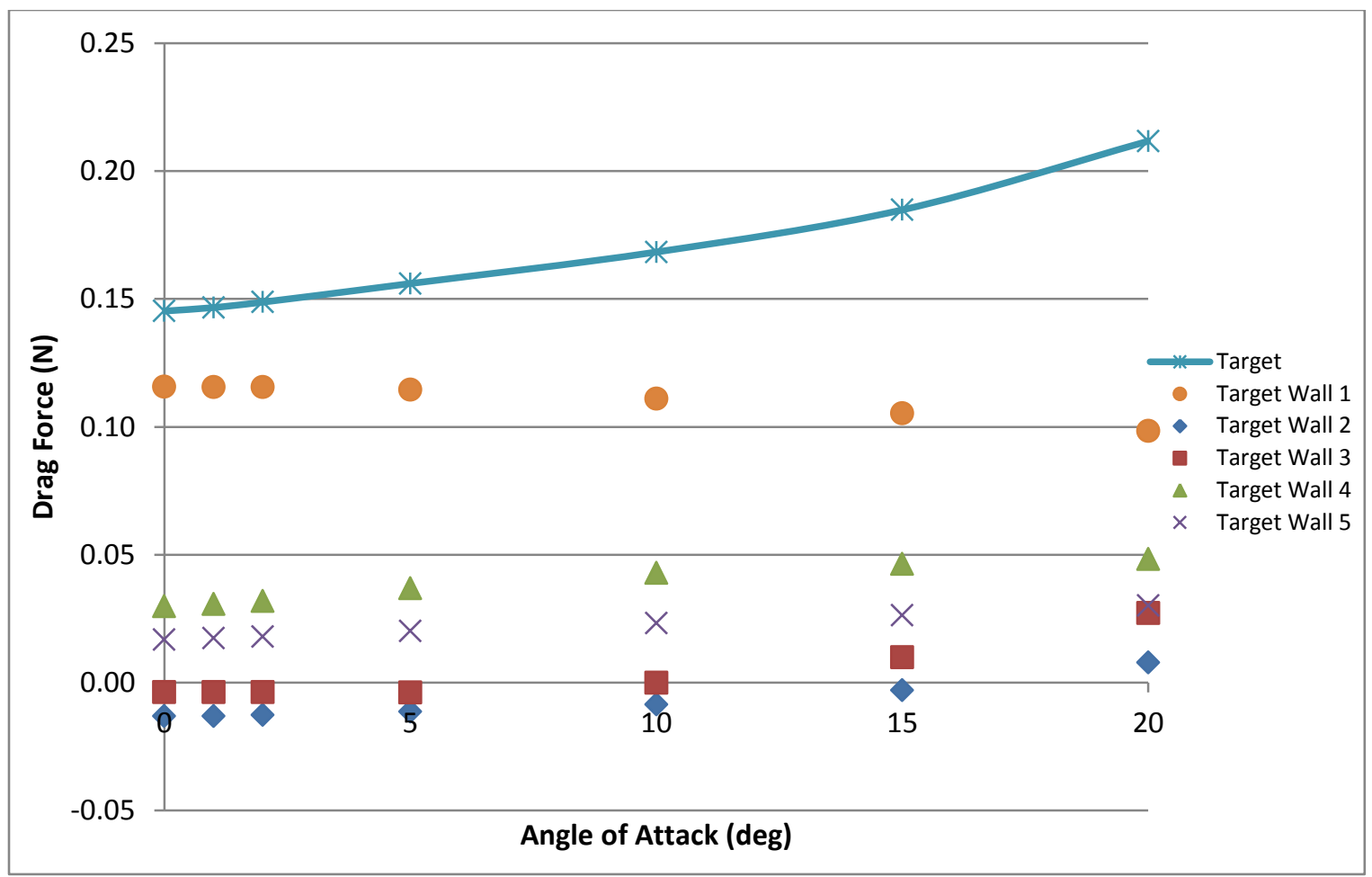

Figure 125. Drag Force on Non-Spinning Target in Drift Region at Different Angles of Attack from 3D, Steady-State Model

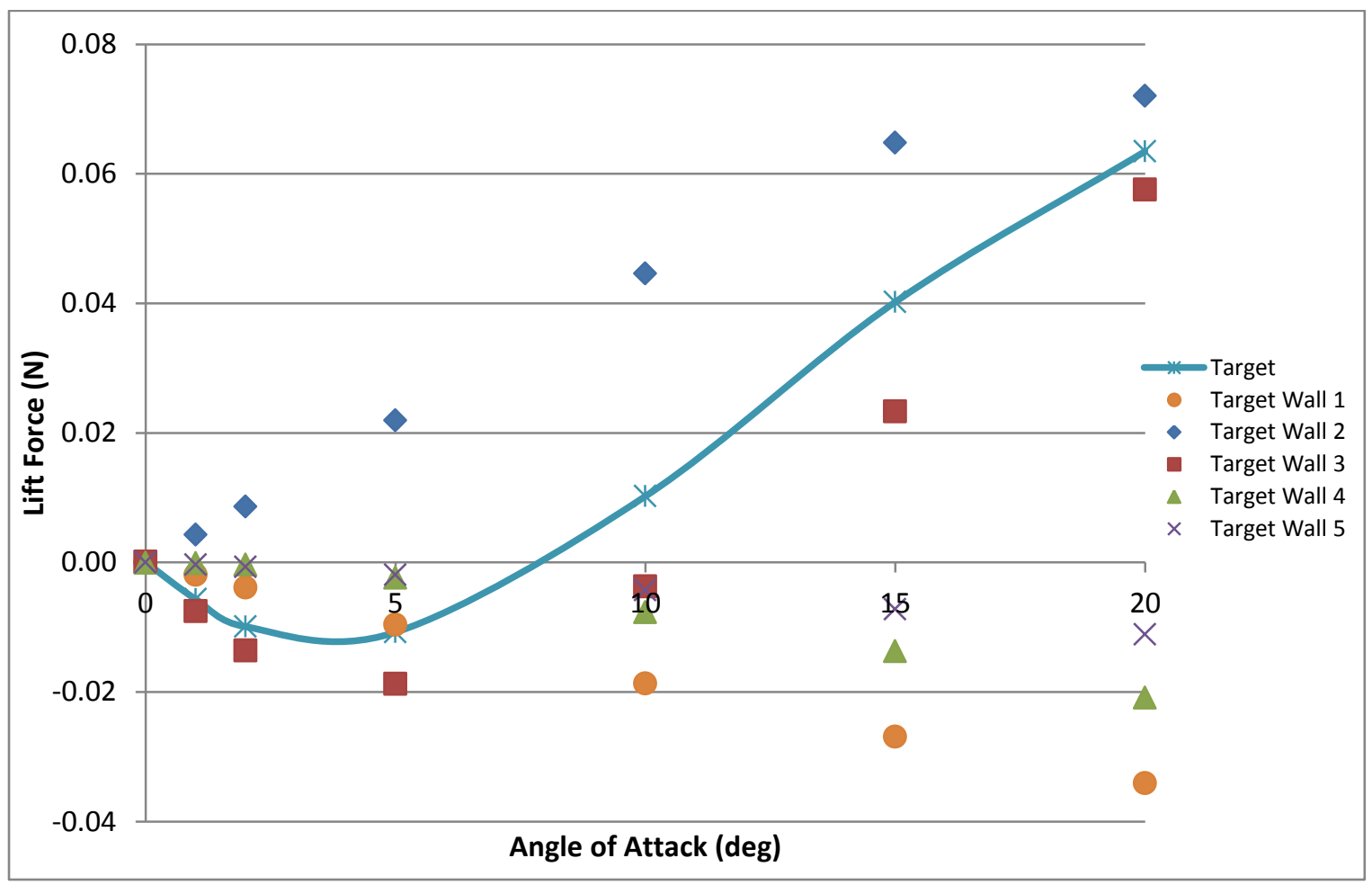

Figure 126. Lift Force on Non-Spinning Target in Drift Region at Different Angles of Attack from 3D, Steady-State Model 


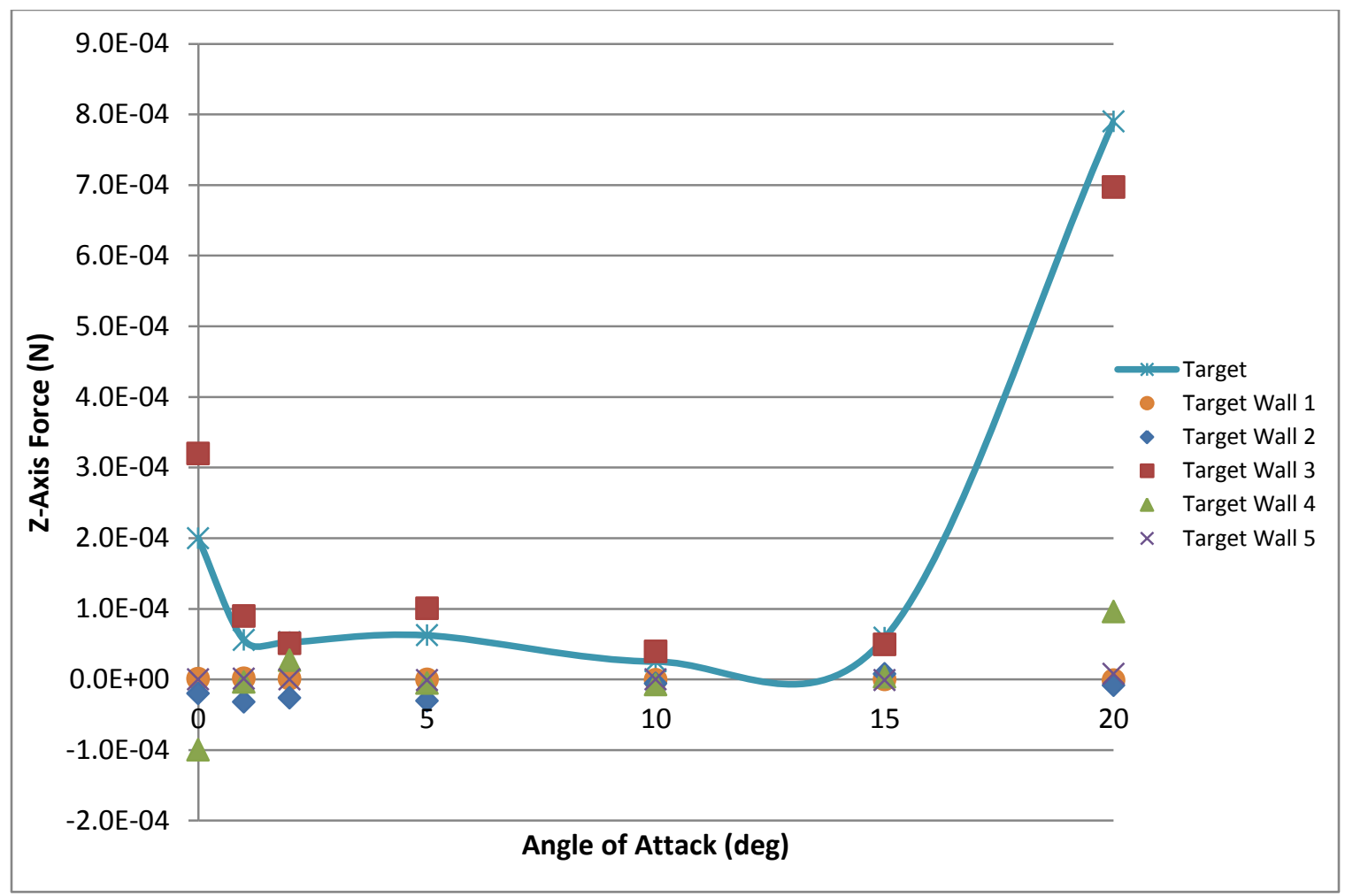

Figure 127. Z-Axis Force on Non-Spinning Target in Drift Region at Different Angles of Attack from 3D, Steady-State Model

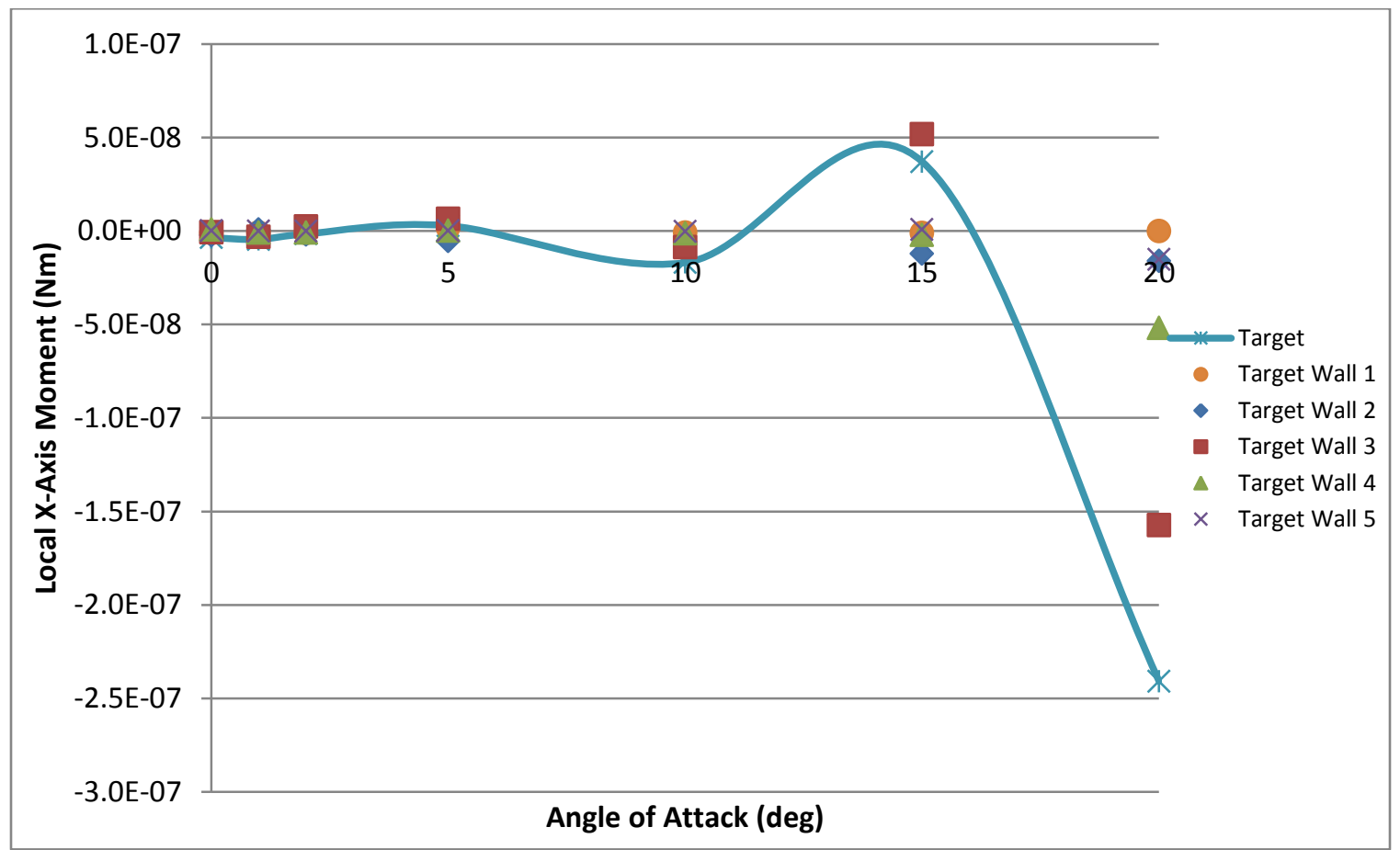

Figure 128. Local X-Axis (Axial) Moment on Non-Spinning Target in Drift Region at Different Angles of Attack from 3D, Steady-State Model 


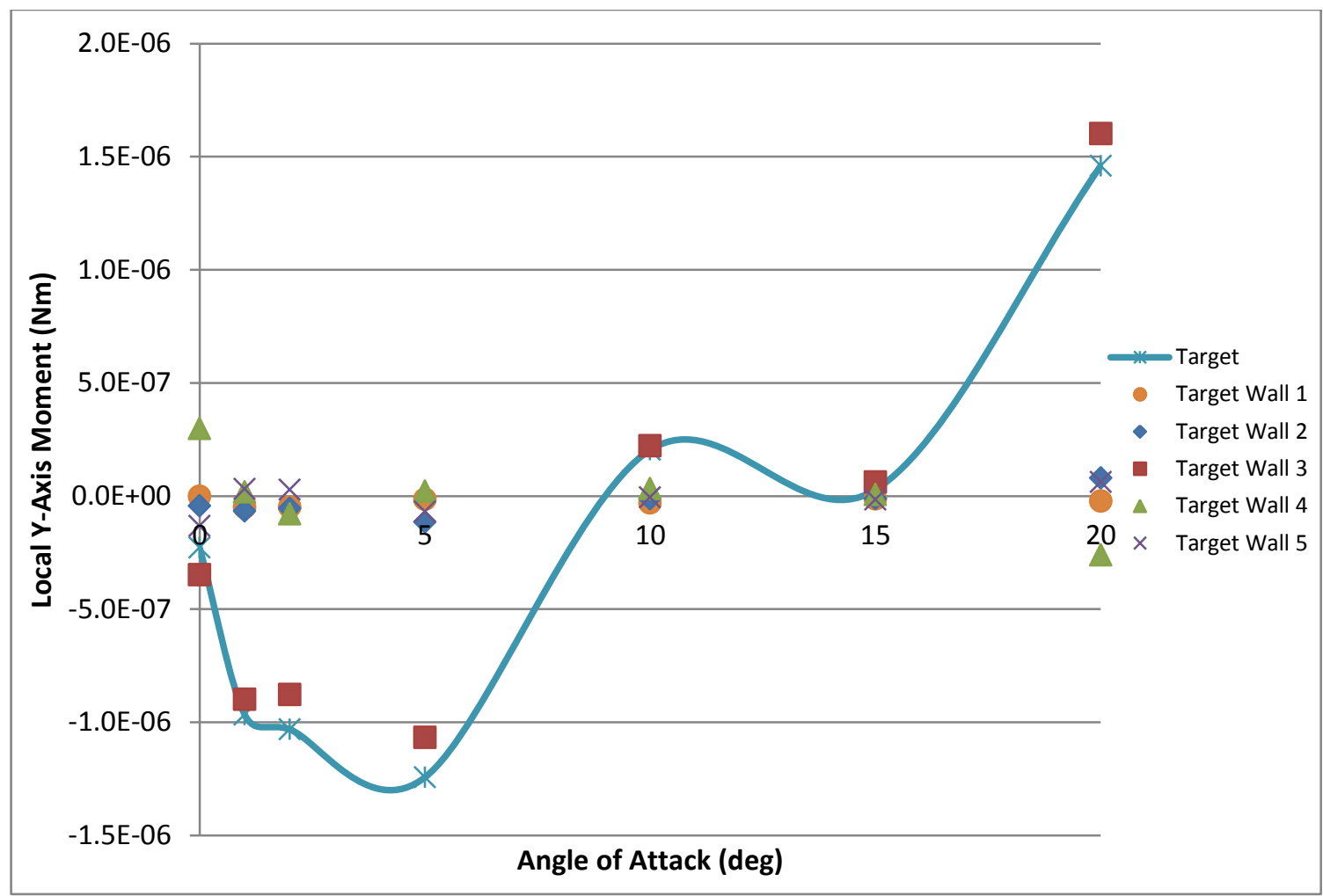

Figure 129. Local Y-Axis (Negative Magnus) Moment on Non-Spinning Target in Drift Region at Different Angles of Attack from 3D, Steady-State Model

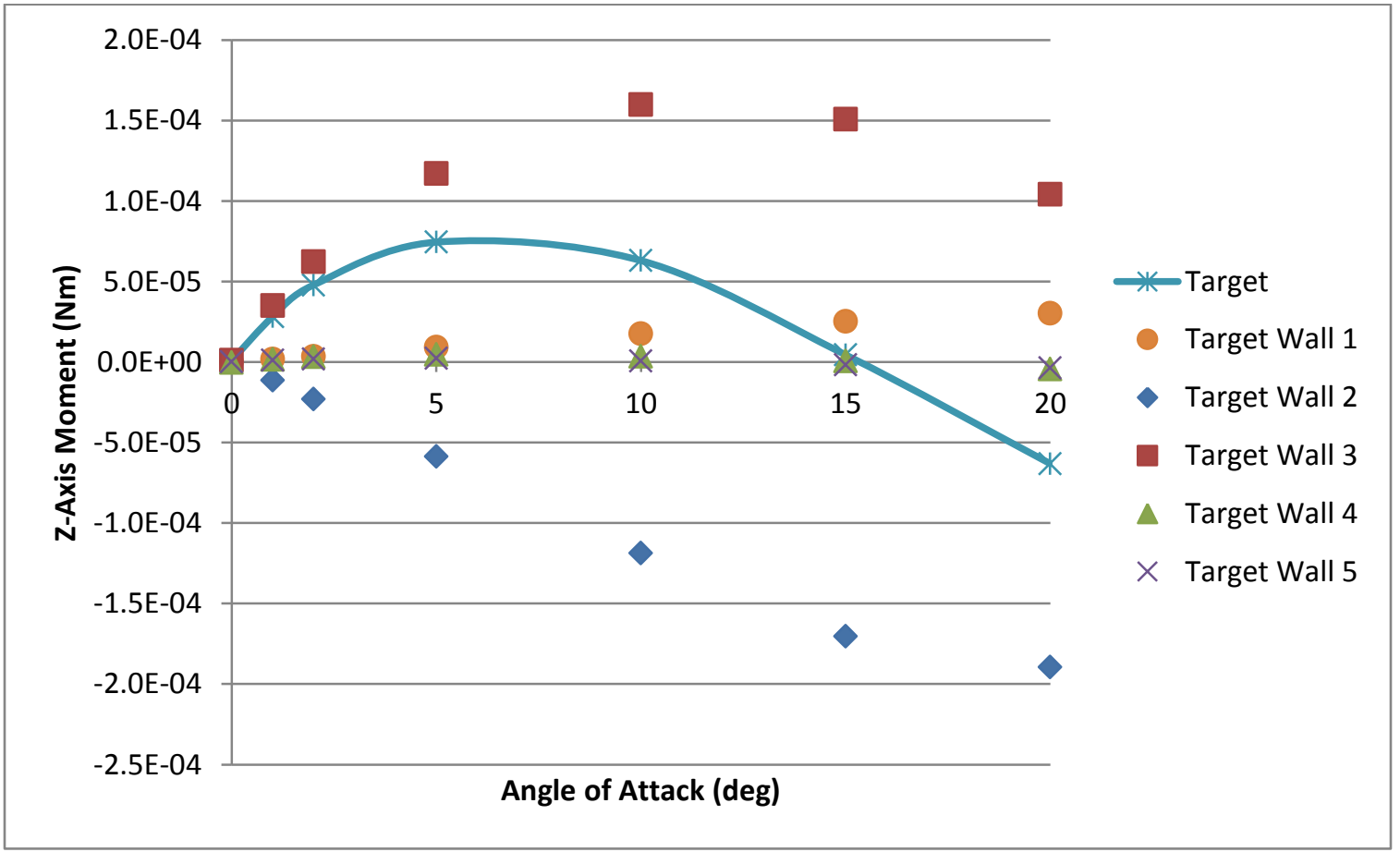

Figure 130. Z-Axis (Negative Overturning) Moment on Non-Spinning Target in Drift Region at Different Angles of Attack from 3D, Steady-State Model 


\section{Appendix D: Angle of Attack MATLAB Code and Plots}

The MATLAB code used to determine the target's angle of attack over time was:

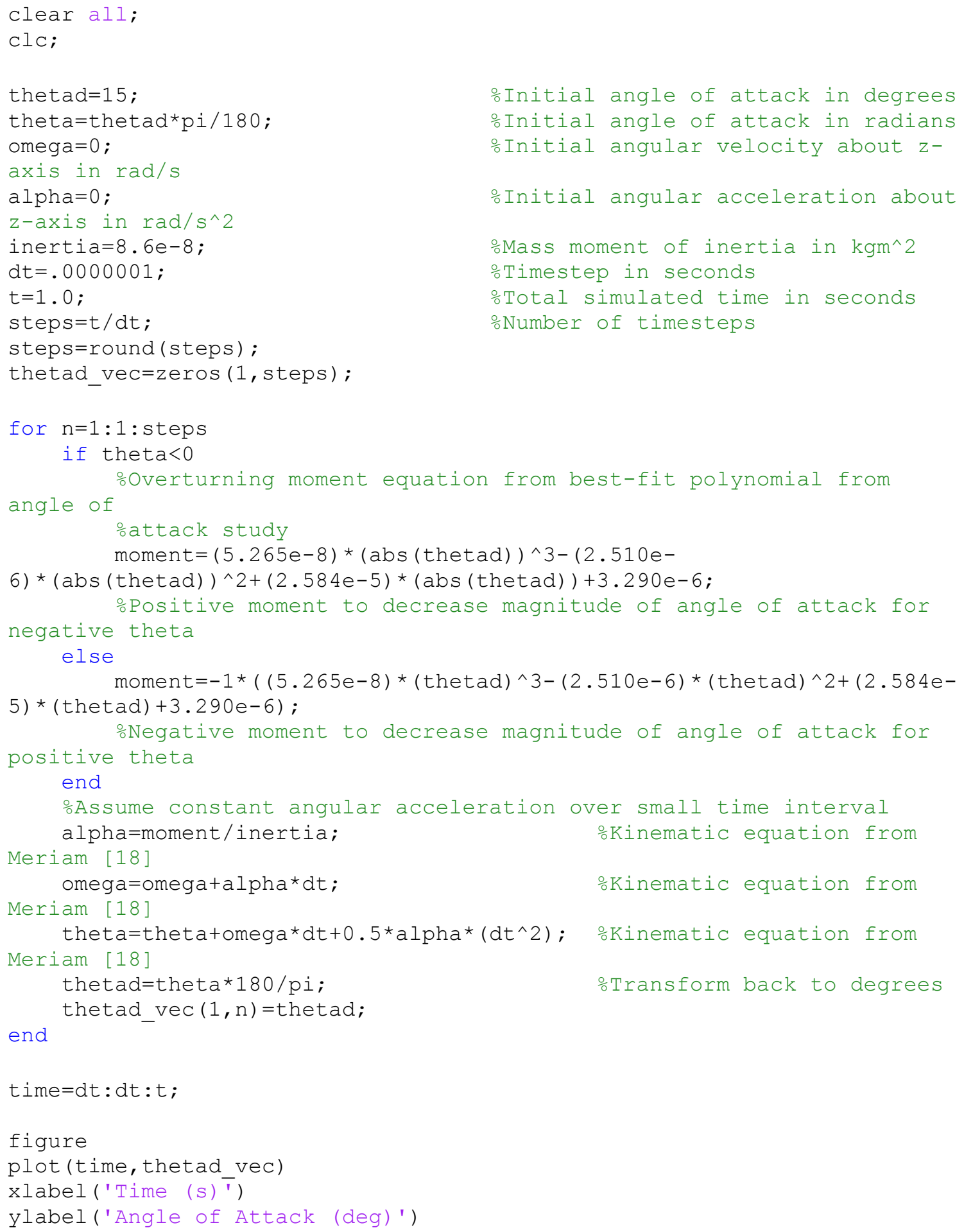




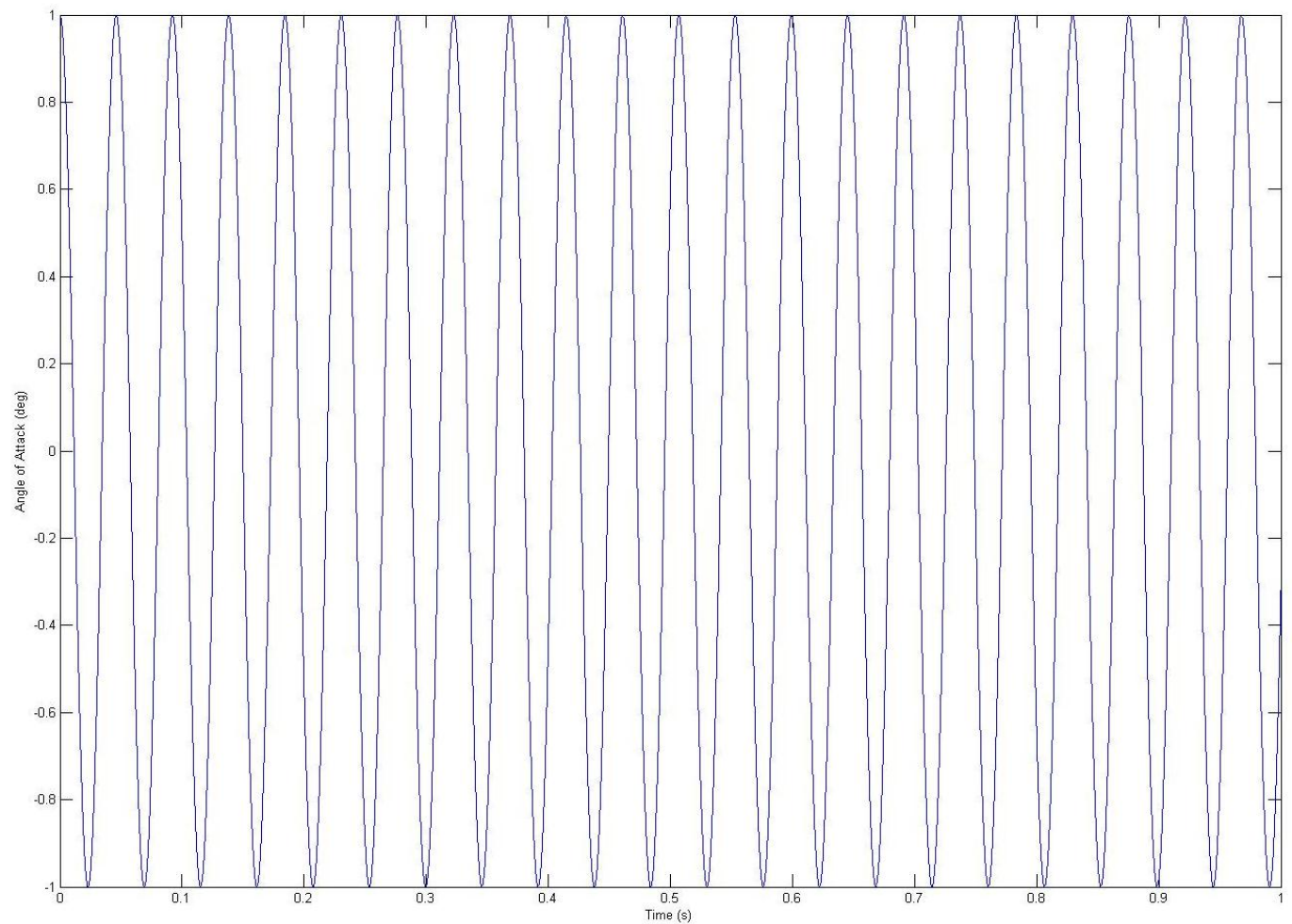

Figure 131. Angle of Attack over Time for Target with Initial Angle of Attack of $1^{\circ}$

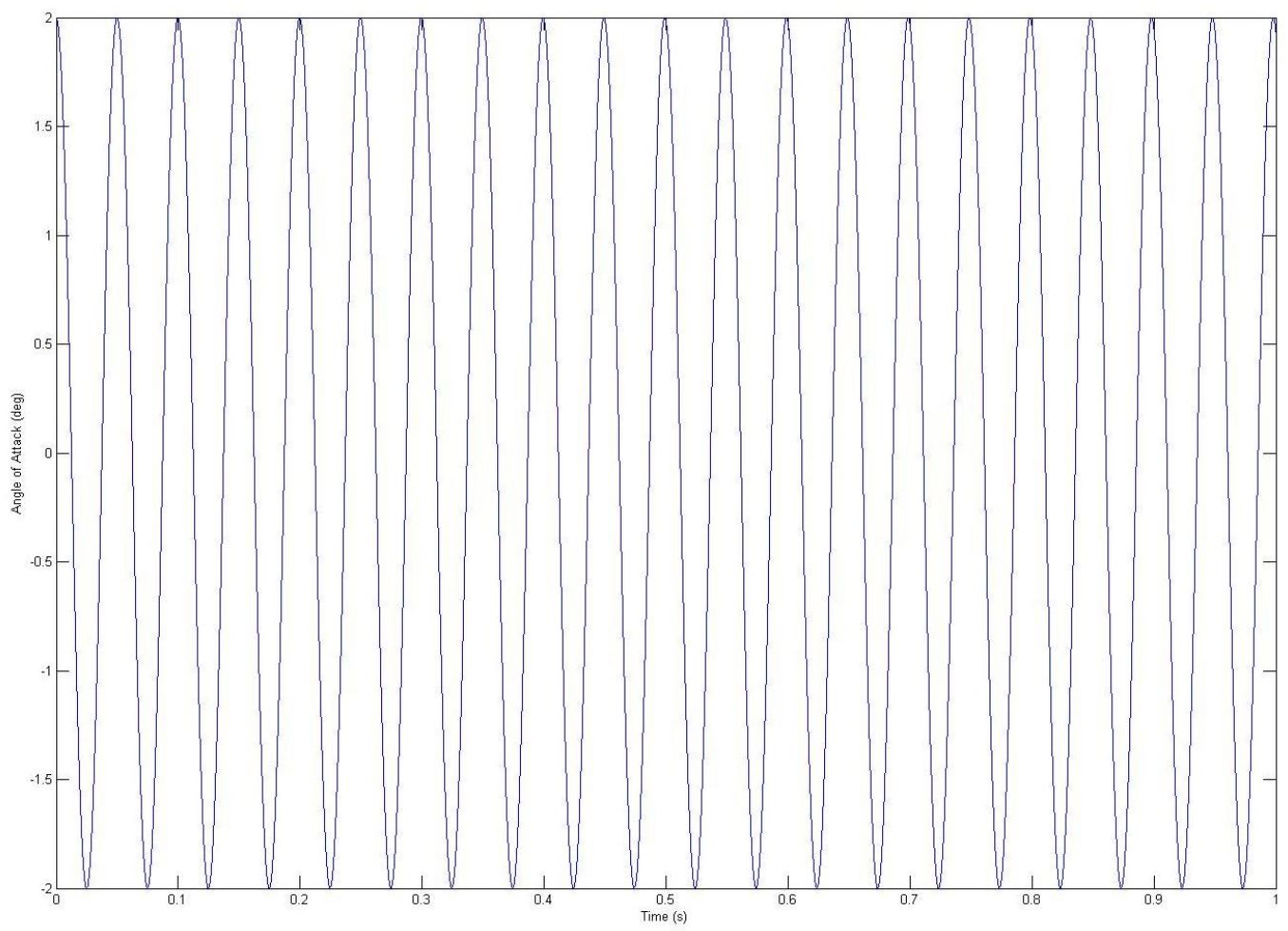

Figure 132. Angle of Attack over Time for Target with Initial Angle of Attack of $2^{\circ}$ 


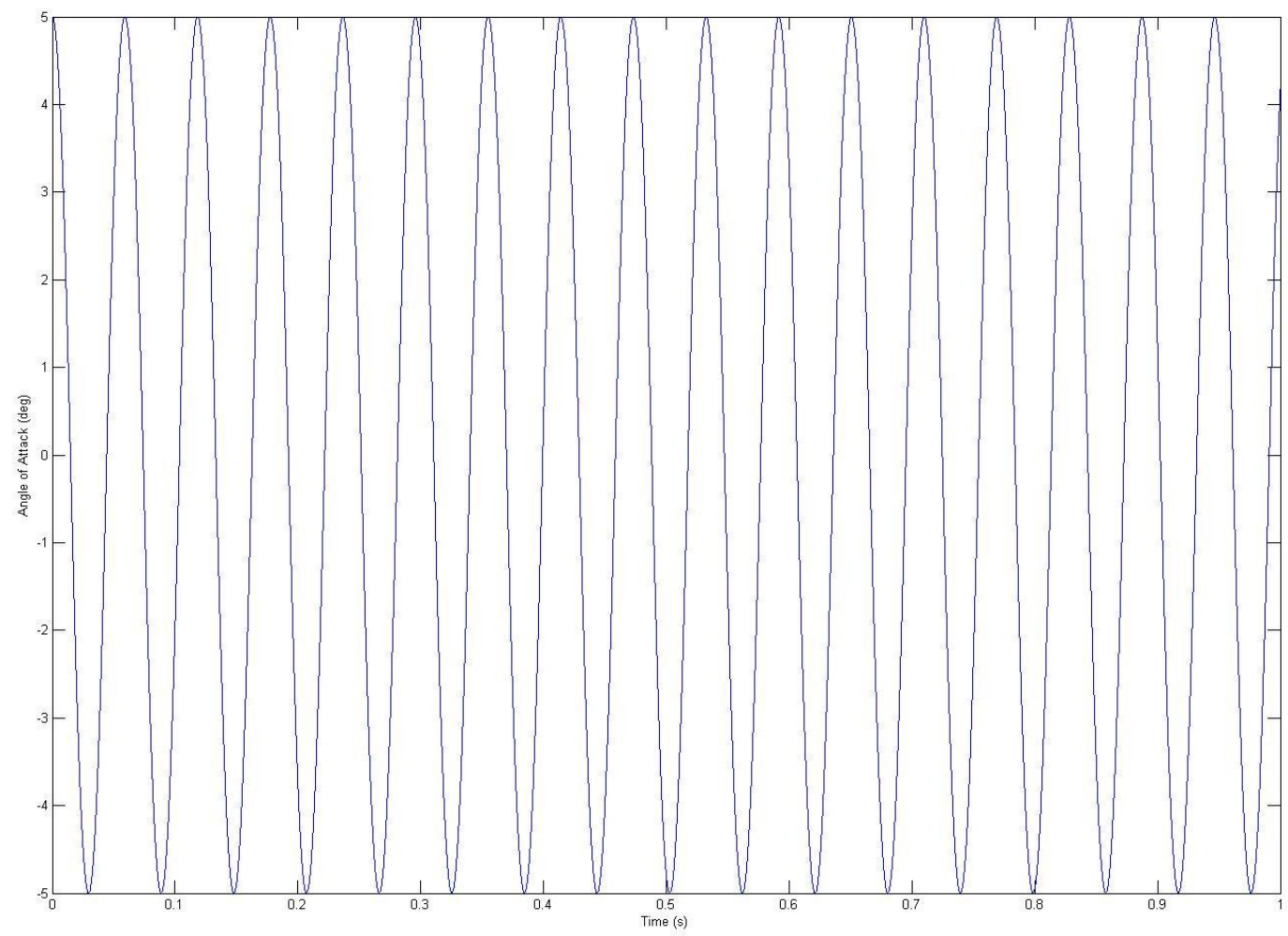

Figure 133. Angle of Attack over Time for Target with Initial Angle of Attack of $5^{\circ}$

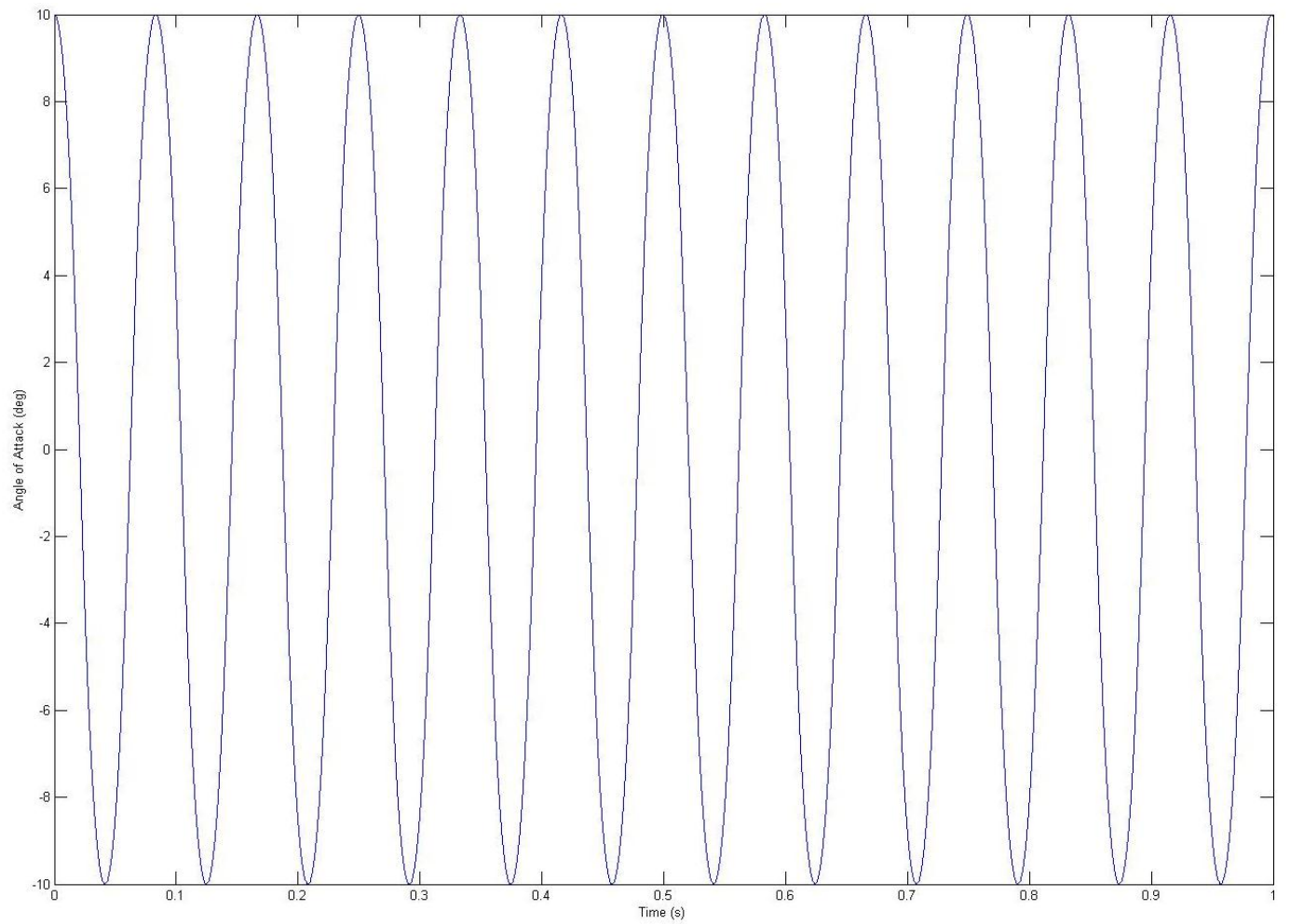

Figure 134. Angle of Attack over Time for Target with Initial Angle of Attack of $10^{\circ}$ 


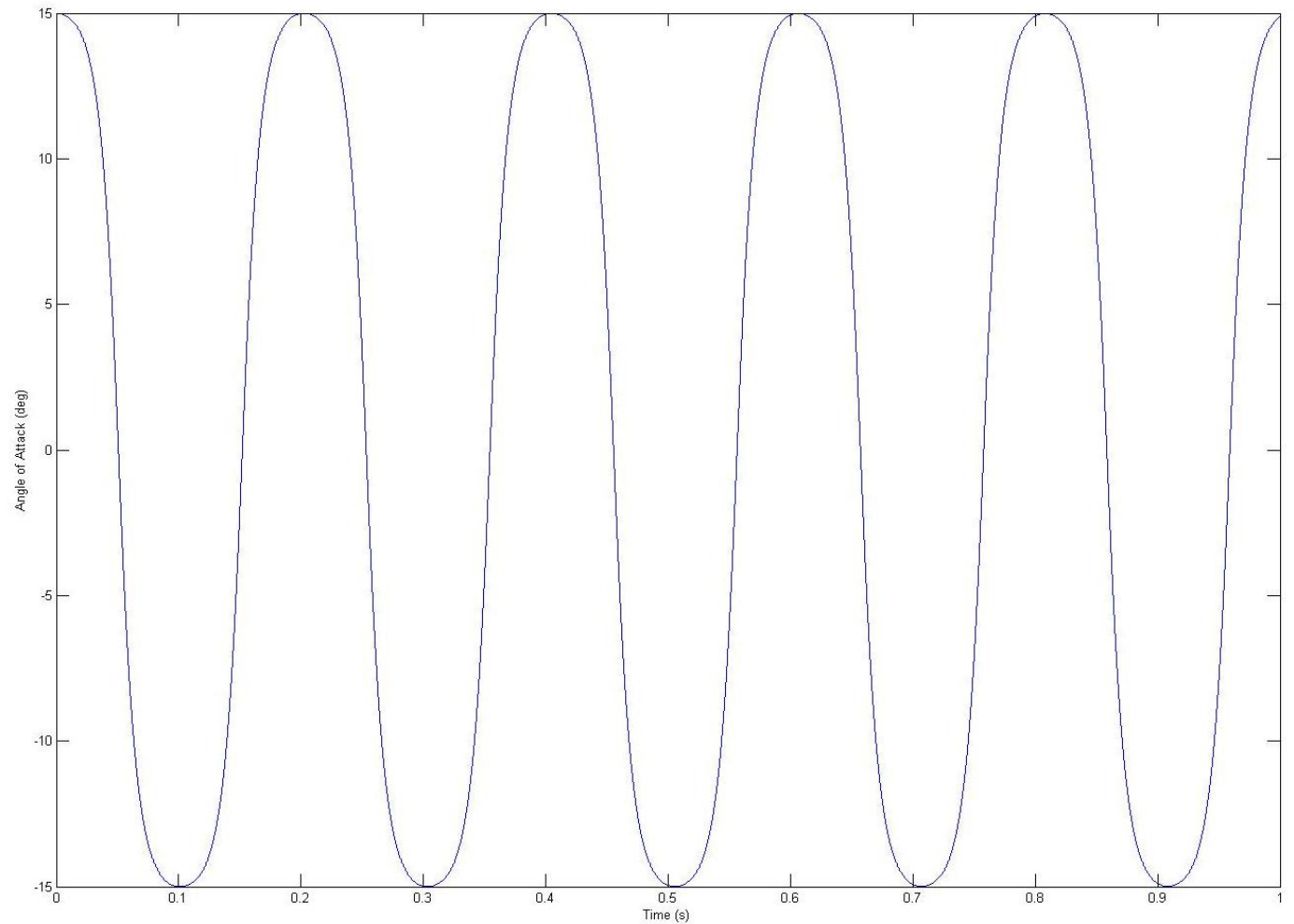

Figure 135. Angle of Attack over Time for Target with Initial Angle of Attack of $15^{\circ}$ 